
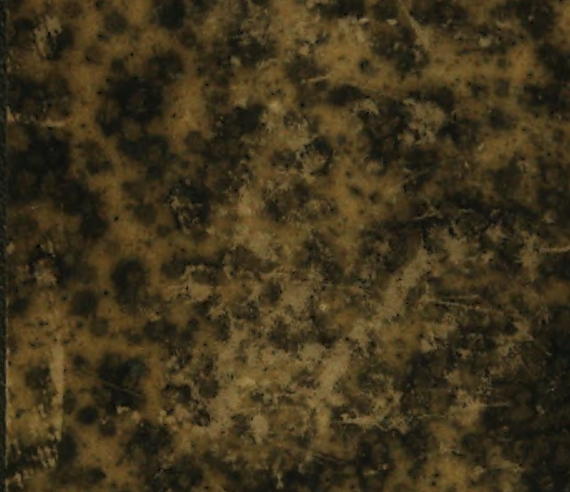

\title{
FRAGILE
} DOES NOT CIRCULATE

son

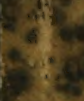

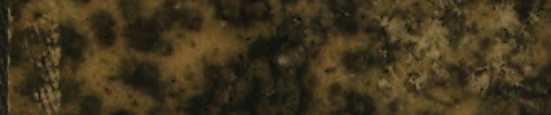



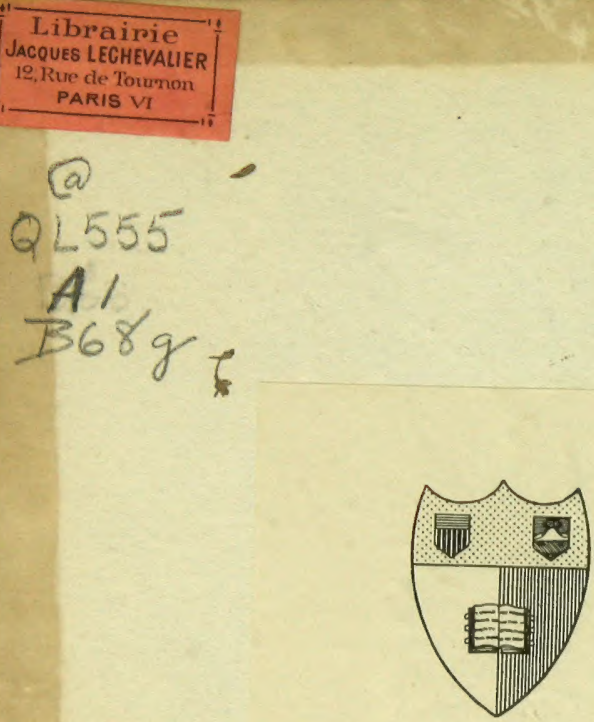

New Hark

State Crallege of Agriculture

At Çnenell Zntuersity

3thasa, N. V.

Jibrary 


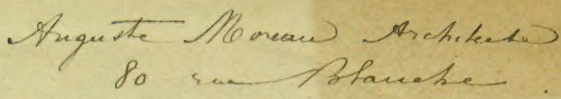

QL 555.A Cornell University Library

$1868 \mathrm{~g}$

Genera et index methodicus europaeorum I

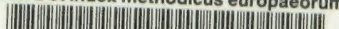

3 1924018543318 

GENERA

ET

\section{INDEX METHODICUS.}




\section{SUITES A BUFFON}

FORMANT, AVEC LES OEUVRES DE CET AUTEUN,

\section{UN COURS COMPLET D'HISTOIRE NATURELLE}

\section{RABRASSANT LES TROIS RËGXES DE LA NATURE.}

Les possesseurs des OEuvres de BUFFON pourront, avec ces Suites, compléter toutes les parties qui leur manquent, chaque ouvrage se vendant séparément, et formant, tous réunis, avec les travaux de cet homme illustre, un ouvrage général sur l'histoire Naturelle.

Cette publication scientifique, du plus haut intérét, préparèe en silence depuis plusieurs années, et confièe à ce que l'Institut et le haut enseignement possédent de plus célébres naturalistes et de plus habiles éerivains, est appelée à faire époque dans les annales du monde savant.

Les noms des auteurs indiqués ci-après sont, pour le public, une garantie certaine de la conscience et du talent apportés d' la rédaction des différents traités.

ANatomie GoMPAnÉ par M.

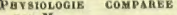
par M.

Céracés (Baleines, Dauphins, eto.), ou Recueil et examen des falts dont so compose l'histoire de ces animanx, par M.F.Curier membre de linstitut, professear an Muséum d'Histoire naturelle, eto, $1 \mathrm{~V}$. in-8 aveo deax lisraisons do planches (Ouvrage noires, $12 \mathrm{fr} .80 \mathrm{o}$; $\mathrm{flg}$. coloriees $\quad 18 \mathrm{fr}$. $50 \mathrm{c}$

REPTILES(Serpens, Lézards, Grenouilles, Tortues, ete.), nar M. Dumérll, membre de l'Institut, prófesseur la Faculté de Miédecine et au Muséum d'Histolre natorelle; st M. Bibron aide-naturallste : 8 vol, e 8 lirraisoes de planches. Les tomes 1 ì 5 et $\mathbf{8}$ son en vente, les tomes $6 \mathrm{et}$ paraltront incessamment.

Porssoks, par M

ExтомoLocis (Introduction a 1), comprenant les principes généraux de l'Anatomie et de la Physiologio des Insectes, des détalls sur leurs mours, et un rés sur leurs mours, et un résumé des princlpaux sy s-
temes de classincation. temes de classincation etc., par M. Lacordaire líge (Ouvrage termine adopte et recommande adopte et recommana par luniversite pour etre place dans les bibliotheques des Facultés et des Colldges det ves); 2 vol. in-8. Figare noires, $19 \mathrm{fr}$; figures colorlées $28 \mathrm{fr}$ Y MECTES CoLtoptÉE: Cantharides, Charancons,
Hannetons, Scarabées, eto.), par $\mathbf{M}$

- Onxuoprénes (Grillons Griquets, Sauterelles ) par M. Sérville, ex-prési dent de la Soclété entomologique de France; vol. aveo planches, Prix 0g. noires, if $\mathrm{fr}, 50 \mathrm{c}, \mathrm{et}$ fig colorléeg is fr. 500 . (i) coloriées,

- IIE mup tहr Res (Cigales, Pu naises, Cochenllles, otc.) par M. Serville.

- LEpidoptênes (Papillons), par M. le docteur holsduval; tome 1 arec 2 Hivraisons de planches. Prix:0g, noires, 12 f. $50 \mathrm{c}$ fo. coloriées, $18 \mathrm{fr}, 50 \mathrm{c}$ - Nḱvaoptéses (Demolselles, Ephémères, ete.) par M. le docteur Rambur.

- НYмÉNOPTÈREs (Abeilles, Guèpes, Fourmis, ete.) nar M. le comte Lepello tier de Saint-Fargeau; tome 1 et uno livraison de planches. Prix : flgures nolres, $9 \mathrm{fr}$. $50 \mathrm{c}$, $\mathrm{fg}$. coloriées, 12 fr.'s0 e. -Dipténes (Mouches, Cousins, etc.), par M. Macquart directeor du No séar directeor du mude Lille; 2 vol. in -8 et cahlers de planches $(O u$ cablers de planches (Ou-
vrage termine $)$ Prix : vrage terminé). Prix
ngares nolres, 19 fr. ; gures coloriées. $25 \mathrm{fr}$. - Aptrares (Aralgnées. Scorpions, ete.\}, par MI. Ie baron Walekenaer, membre de IInstitut; tome t aveo 3 cahiers de planches. Prix : lig. nolres $15 \mathrm{fr}, 50 \mathrm{c}$.

0ig. coloriées, $25 \mathrm{fr}$. $150 \mathrm{c}$.

Le come 8 el dernier paraitra en 1840. maris, Crabeto. prenant IAnatomis Physiologie et la classin catlon de ces Anlmaux par M. Mllne - Edwards membre de l'Instítut, proresseur d'hlstoire naturelle, ete, ; tome 1 et? aveo 2 Ilyraisons de planches. Prix : fig. noires $19 \mathrm{fr}$; figures coloriées $25 \mathrm{fr}$.

Le tome 3 et dernier pa raitra en 1840.

Moszuspoes (Moules, Hultres, Escargots, Limaces. Coqullles, eto.), par M. de Blainville merobre de Niustitat, professeur an Muséum d'Histoire naturelle, ete.

AพxéLrogs (Sangsues, eto.) par M.

VEAs xNTE STINAUx (Ver Solitalre, ete, par M.

ОоР И Т Е S AGALẼPHES

(Physale, Béroé, Angele. ete.), par M. Lesson, correspondant de I'Institut pharmacien en chef de Marine, a Rochefort.

- Echivode RaEs (Oursins

Palmettes, etc.), par II.

Lacordaire, professeur

d'histoire naturelle Liége.

- Polypiers (Coraux, for gones, Éponges, etc.), par M. Mline-Edwards, membre de l'Instltut, professeur d'hist. naturelle, etc

- Ingusoures (Animalcules mieroscopiques), par M. Dujardin, professeur d'histoire naturelle Toulouse.

BOTANiove (Introduction a l'Etude de la), ou Tralté élémentaire de cette sclen- ce, contenant l'organographie, is Physiologie etc., ete., Dar M. Alph. de Candolie professeu dhistoire naturelte a Genève (Ouvrage termine autorise par VUniver site pour les Colléges royaux et commu naux) $; 8$ \& et un cabier de planches. Prix : $16 \mathrm{fr}$

VÉgétaux pHanéRooame (À Organes sexuels apparents, Arbres, Arbrisseaux, Plantes d'agrément, oto., par M. Spach. alde-naturaliste an Mitalde-naturatisto au missetim dist. natureile. sons de planches. Prix figures noires, $81 \mathrm{fr}$. $80 \mathrm{c}$ fig. col. $117 \mathrm{fr}$. $50 \mathrm{c}$

- Cryptocames (a Organes sexuels pell apparents ou caches Mousses Fougères, Lichens, Champlgnons, Truffes, ete.), par II. de Brebisson de Falaise.

GÉloes (Histoire, Forma tion et Disposition de Matérlaux qui composent l'écorce du Globe terres tre), par M. Huot, membre de plasieurs Soclétes savantes; 9 vol. ensemble de plus de 1,500 parges (Ouvrage termine).Prix, avee un Atlas de 26 plan-
ehes, HivénA locie (Plerres, Sels Metaux, etc.), par MI. Alex. Brongniart, membre de Insitut, men. bre de sear au Maseam dist. naturelle, ete., eto, ; et M. Delafosse, maitre des conférences à l'ícole Normale, aide-naturaliste, ote, au Nusénm d'Histolre naturelle.

CONDITIONS DE, LA SOUSCRIPTION :

Les Surgs a Buprox formeront 53 vol. in-8 environ, ment, chaque anteur s'occupant depuis longtemps de Imprimés avec le plas grand soin et sur beau papier; ce la partie qui lui est confée, J'éditeur sera a même de tmprite publier en peu de temps la totalité des traités dont T'étendue convenable. Alnsi qu'll a été dit précédem-

En mai 1840,28 volumes sont en vente, avec 37 livraisons de planches.

Les personnes qui roudront souscrire pour toute la Collection auront la liberté de prendre par portion jusqu'a ce qu'elles soient au courant de tout ce qui est paru.

POUR LES SOUSCRIPTEURS A TOUTE LA COLLECTION

Prix du texte, chaque vol. (1) d'environ 500 à 700 pag., 5 fr. 50 c. - Prix de chaque livraison d’environ $10 \mathrm{pl}$. noires, 3 f.; coloriées, $6 \mathrm{fr}$.

Nors. - Les Personnes qui souscriront pour des parties séparées, payeront chaque rolume $6 \mathrm{fr}$. $50 \mathrm{c}$ Le prix des rolvmes papier vélin sera double du papler ordinaire.

(1) L'Ébiteur ayant à payer pour cette collectlon des honoraires anx anteurs, lo prix des volumes ne peut étre comparé à celul des réimpressions d'onvrages appartenant au domaine public et exempts de drolts d'anteurs tels que Buffon, Voltaire, ete, etc.

ON SOUSCRIT, SANS MIEN PAYER D'A VANCE, A LA LIBRAIRE ENCyclopedoue DE RORET, Eoiteur de la Collection de Manuels, du Couns d'Agriculture au XiXe siécle, etc., RUE Hautragulle, $10 \mathrm{bis.}$ 


\title{
GENERA
}

ET

\section{INDEX METHODICUS}

\section{EUROPEORUM LEPIDOPTERORUM,}

\author{
A \\ DOCTORE J. A. BOISDUVAL, \\ Equite Legionis Honor.; Academla Imper, et Regal. Florenun.; \\ sociat. Imper. natural. hlstor, Mosquens.; Lycat Amerio. bor.; Societ. Eatomolog. Londin. \\ Soclet. Entomolog. Gall. Prosid. vices gerent., \\ Recuon pluriam Societ. hist. natural, aut medicins sodali.
}

PRIX : 5 FRANCS.

\section{PARISIIS,}

APUD RORET, BIBLIOPOLAM, Via Gautefeuille, to bis.

1840. 
(a)

QL555 Al $B 68 \mathrm{~g}$

(218088

EX TYPIS FAIN ET THUNOT,
vid Ragine, 28. 


\section{PRAMONITUM LEPIDOPTEROPHILIS.}

Ante hos undecim annos, libellum in publicam lucem edidi, cui titulus est : Index Methodicus. Hune apud rei insectoriæ studiosos benigna existimatione acceptum, pleræque in Europa collectiones, ut ordinarentur, ducem et auctorem secutæ sunt. Post id tempus, tum novas species, multas numero, usus reperit; tum multas etiam, veteri jamætati cognitas diligentiori cura, investigatorum labor dispexit, ut libellus ille noster hodiernæ scientiæ non respondeat.

Frequenter autem rogatus sum ut altera editione opusculum ipsum publicarem, is tantummodo rebus auctum, quæ novissime inventæ accesserunt. Sed quum majoris quædam eomprehensionis, altiorisque investigationis, quæ ad universa Lepidoptera spectant, præparaverim, inde existimavi utilius fore si Genera ederem, quam si Europæas species methodica enumeratione, ut prius, recenserem et lectoribus exhiberem.

Omnino igitur novus est quem propono liber, continetque Familiarum, Tribuum et Generum characteres, adjuncta diagnosi, quæ ad recentiores species pertinet. Sicut in catalogo quem MDCCCXXVIII anno publicavi, exoticis tribubus generibusque rursus manum 
admovi; unde illud Genera non universam meæ methodi rationem exhibet, sed excerpta quædam, quibus Europææ duntaxat species tractantur.

Itaque eorum causa qui, citra alias, in harum studio potissimum versantur, opusculum illud scriptum est.

Quodquidem, licet tenue et exiguum sit, nescio an, multis partibus imperfectum esse videatur, plus a me temporis et investigationis postulavit quam duo mei Species Général volumina. Enimvero uniuscujusque speciei, apud omnes auctores qui eam verbis aut figura expresserunt, singulos habitus inspexi et ad eorum fidem comprobavi, quo exactius et exquisitius eam per synonyma sua ducendo exponere possem.

Vetustis quidem et exoletæ jam memoriæ nominibus abstinui, quibus species plane notæ designantur; eorum vero quæ, nuper creata, nuper repertas species designant, nullum neglexi, eo quod primum tempore erat, assumpto semper et usurpato.

Junctum mihi commercium cum probatissimis apud Germanos entomologis; id attulit ut ex visu cognoscerem plerasque typicas species a clarissimis viris Ochsenheimer, Treitschke, Hubner, Freyer, etc., descriptas vel figuratas, aut a D.D. Dahl, Parreys, Kindermann, Frivaldjsky, etc., repertas. Sueci entomologi plurimas arcticarum regionum species milii visendas præbuerunt ; et ille indefessus Anderregg, cui debere se prædicat entomologia tot novas species, aut certe non vulgares, ab illo in Valesia inventas, ad plurimarum me cognitionem admisit, quibus caret meus Index Methodicus, et circa primas illarum habitus optima mihi documenta subministravit. Gallorum quoque Entomologorum, officio- 
sissima voluntate aperta mihi collectiones fuerunt, aut communicatx. Qui quidem illud gratx recordationis testimonium tum universi acceptum habeant velim, tum singulariter ac pracipue eruditissimus D. Rambur et oculatissimus D. Daube, qui, si quando novitates invenerant, in earum partem semper largius me vocaverunt, et haud mediocrem rerum accessionem ad id ittulerunt ut europxa Fauna amplificaretur.

Singulas autem notas arithmeticas, hoc a multis entomologis rogatus, ad species apposui, quo faciliorit essent corum inter se commercia, si eis nomina, vel sape synonyma in sua desiderata et missionis catalogos referendi labor tolleretur.

Primum in animo habebam, aliquantum cunctando, universam Lepidopterorum Europa molem semel complecti et exhibere, sed entomologorum multi, opusculum nostrum manibus versare cupientes, ab hoc proposito dehortati sunt. In posterum tamen reliquam Lepidopterorum seriem, ni deficiat vita, complere et methodice ordinare satagam.

Parisiis, 1840.

\section{J. A. BOISDUVA I}




\section{LXPLANATIO SIGNORUM}

\section{NOMINUMQUE ABBIEVIATOIUU.}

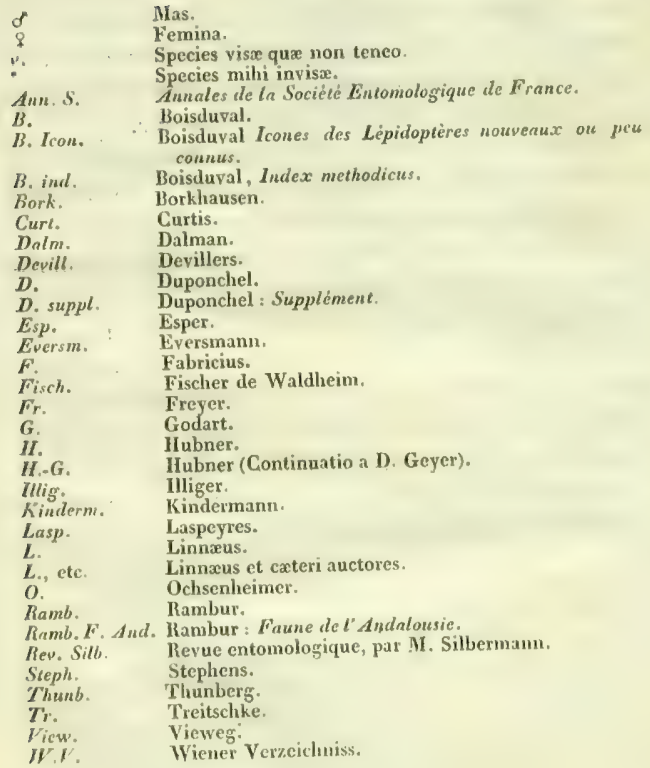




\section{MONITUM.}

Typis dum opus excuderetur, dux Species omissæ sunt in Indice; lector igitur benevolus eas inserere rogatur:

\section{GENUS DEILEPHILA.}

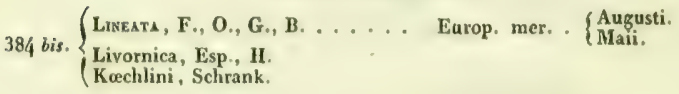

GENUS PSYCHE.

632 bis. Pluagerelza, H.213....... Gallia bor. . Julii.

\section{CORRIGENDUM, p. 154.}

\section{GENUS CUCULLIA.}

$1237\left\{\begin{array}{l}\text { Splendroa, Cr. 400, F. . . . Russ. mer. } \\ \text { Argyrea, Kinderm. in Li } \\ \text { An Lactea P D. Pap. de France. }\end{array}\right.$

Notn. Nomen Cramerianum, ut multo antiquius, usurpandum et sane servandum. 



\section{LEGIO PRIMA.}

\section{RIIOPALOCERA.}

Antennx plus minusve ad apicen clavata. Alæ omnes insecti quiescentis erectæ, liberx; retinaculo nullo. Stemmata nulla. Volatus diurnus.

\section{SUCGINCT压.}

Chrysalides ano filoque transverso alligatx.

\section{TRIBUS PAPILIONIDES.}

A.arva collo tentaculatx. - Mac posticx marcine interiore pro alulonine libero excise. Areola discoidalis clausa

1. GENUS PAPILIO, Lat., Och., Boist.

Clava antennarum sub-arcuata. Palpi brevissimi, catpite bresiores, ul-solete articulati; articulo tertio inconspicuo. Ale postica in nostratibus caudatæ.

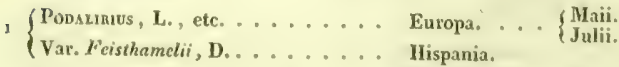

$2\left\{\begin{array}{l}\text { Alexsnon, Esp., G., B., If., Tr. ... } \\ \text { Polydamas, Deprunner. }\end{array}\right.$

3 Hospiton, Gené. .......... Sardinia.... Maii.

Affinis P. Machaon, at multo nigrior et planc distinctus; alis anticis subtus. fascia fusca, sub-marginali, acute dentata, maculis marginalibus ochrit- 
ceis sagittatis ; posticis supra arcu analifulvo, lunulisque marginalibus. -Iarya viridis, nigro, albido rubrorue punctata, spinulis obsoletis instructa, forulam communcm pascens. In Sardinia detexit Cl. Gené

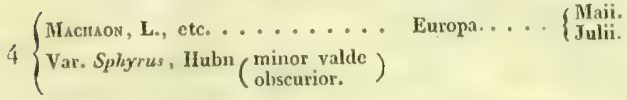

$5 \operatorname{Xutuus}(1)$, L. ........ $\begin{aligned} & \text { Russ, orieut. } \\ & \text { Sibiria. }\end{aligned}$

\section{GFNUS THAIS, Fab., Lat., Boisd. \\ Zerynthia, Och.}

Clava antennarum sub-arcuata. Palpi capite longiores, hirsuti, distincte tri-articulati. Ala deutate, ochraces, nigro sanguineoque maculata.larva arislolochiis vescentes.

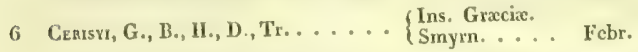

7

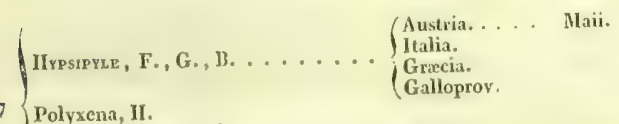

Var. Demnosia, Daht.

Var. B. (alis fulvo - ochraceis).... Moraca.

Var. Cassandra, II. ........ Galloprov.

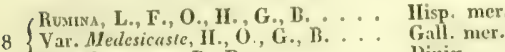

ILisp. mer... Maii.

Ab. Honuoratil, B., D. ....... Dinix.

(1) In hoc opusculo speciss Sibirix complexus sun, quia pluisque cotomologis lillropes babentar species, quecumine in Rossic is Asix borealis poscesionibus occurrubl; Exempl. G: Aurora, Narica, Tarpeia, ete. 


\section{GENUS DORITIS, Och., Boisd. \\ Thais, God.}

Antennx breviores; clava antennarum sub-arcuata. Palpi hirsutissimi, vix capite longiores, obsolete tri-articulati. Alx integra sub-desquamatx, sub-membranaceo-rugulosa.

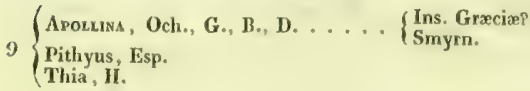

4. GENUS PARNASSIUS, Lat., Boisd.

Dorites, Fab., Och.

Antenux lreviores; clava sub-ovata recta. Palpi capite longiores, frontem ultia assurgentes, pilis longis limbriati, distincte tri-articalati. Ala integerrima, rotundata, subtus et ad marginem denudatic. Anus femina valvula magna, coriacea, tectus.-C Cluysalis suls-folliculata, mores Hesperidarum revocans.

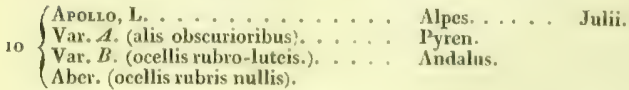

11 Nomon, Fisch., B. . . . . . . Sibiria.... Julii.

1.2 YMoerds, G., B., II.......... Mpes...... Julii.

Delius, Esp., O. .......... Russia.

13 Conyoas, Fisch., B. ........ Sibiria or.

14 Mnemosłne, L., etc........... Alp. Lur. ... Junii. 


\section{II. 'TRIBUS PIERIDES.}

Larve pubescentes, antire posticeque attenuatx. Chrysalides angulosx, antice mucronatx, - $\mathrm{Al}$ ie postice pro receptione abdominis canaliculatx; area discoidalis clausa.

- Palpi rix compressi, graciles, pilis raviusculis hirti, articulo ultimo lineari. Antennce nigrce.

1. GENUS PIERIS, Boisd.

Pierides, Latr., Pontia, Ochs.

Chrysalides articulis mobilibus. - Antenna clava ovoidea, capite thoraceque longiores. Ale sub-trigone; areola discoidali posticarun magna, ultra medium extensa.

$+$

15 Chategr, L., etc.......... Europ, .... Junii.

tt

16 Brassick, L.. etc. ......... Europ..... Perannat.

Rape, L., etc. ....... Europ. . . . Peramat.

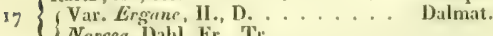

(INarcea, Dahl. Fr., Tr.

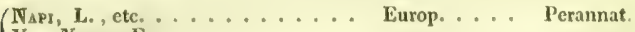

18 Var. Napere, Esp.

Var. o Bryonice, G.

Var. Sabellica, Steph.

ttt

19 Carudrce, Esp., H., O., B., G.... Alpes..... Julii.

20 Chrontore, Fisch., O., II., B., D. . Russ. m. . . Julii.

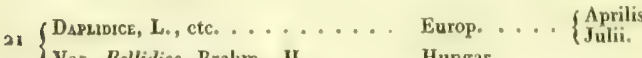

Var. Bellidice, Brahm., II. ..... Hungar. 


\section{GENUS AINTHOCHARIS, Boisd}

Picrides, Latr., Pontice, Ochs.

Chrysalides elongatx, postice anticeque acıminatx, segmentis obsoletissimis, vix conspicuis, immobilibus. $-\Lambda$ ntennx breves, vix capitis thoracisque longitudine, clava brevi capitata. $\Lambda l_{x}$ sub-trigonæ; area discoidali posticarum magna, ultra medium extensa.

+ Alx in utroqne sexu albidx.

22 Grauce, Illig, G., B., D...... Hispan. ... Aprilis.

23 Belema, Esp., H., O., G., B..... Hispan. ... Februarii.

$24\left\{\begin{array}{l}\text { Tagrs, Esp., II., O., B. Icones.... Lusitan. } \\ \text { Bellezina, B. Index, Dup. .... Galloprov. . . } \\ \text { Baii. }\end{array}\right.$

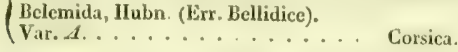

$=\left\{\begin{array}{l}\text { Beuı, F., Esp., II., O., B., G. ... Gall. mer. . } \\ \text { Var. A. (macul. margarit, elongatis.). }\end{array}\right.$

(Var. B. (obscurior.)....... Ilispania.

26 Avsomis, Esp., II., O., B., G. .... Gall. mer. . J Junii.

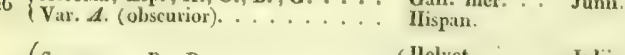

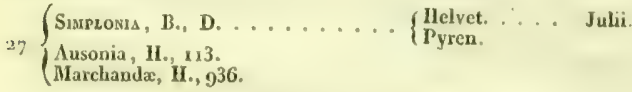

†t Alæ anticæ maris apice fulva.

28 Eupneno, L., etc. ........... Europ. mer. . $\begin{aligned} & \text { Aprilis. } \\ & \text { Maii. }\end{aligned}$

29 Damone, B. . . . . . . . . . Sicilia. ... Martis.

30 Calidamings, L., etc. ........ Europ. .... Vere. 
3. GENUS ZEGRIS, llambur, Boist.

Chrysalides, tela fugaci, tenuissima sub-involutx, contracta, gibbosa , medio sub-strangulatx, antice obtuse mucronata, postice attenuatosub-arcuata, segmentis obsoletissimis, immobilibus. - Antenna rigida, breviuscula, abrupte capitatie. Alix sub-trigona ; areola discoidali posticarum magna, ultra medium extensa.

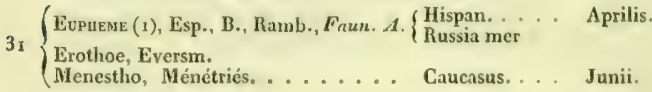

32. Pияотнов, Eversm. ......... Orenbourg... Aprilis.

\section{GENUS LEUCOPIIASIA, Steph., Boisd.}

Pieris, Lat, God,, Pontia, Och.

Chrysalides elongatx, segmentis mobilibus. - Antenna breves capitatx. $\Lambda$ la clongat $x$, albida, tenues; areola discoidali posticarum brevissima, basi vicina.

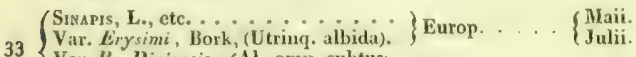

$\left\{\right.$ Var. B., Diniensis, ( $\left.\begin{array}{l}\text { Al. omn. subtus } \\ \text { immacul. }\end{array}\right)$ Dinia.

34 Lathyr , H. , D., B. ....... Gall, mer. . \{ Malii.

" Palpi valde compressi, approximnti, piloso-squamosi, articulo ultimo brevissimo. Antennte rubre.

5. GENUS RHODOCERA, Boisd., Dup.

Gonopteryx, Leach, Colins, Lat., God,, Och.

Chrysalides arcuata, medio gibhosa. - Antenna breves, truncata, sensin crassiores, arcuatic. Mla angulate, flava, subtus puncto centrali argenteo destitutze.

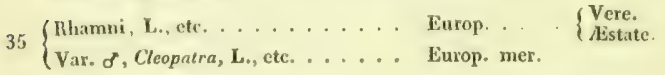

(1) Specimina e Caucaso et Russia meridionali differunt ab individuis Jectis In Itiipana a Cl. Rambur, statura paulo majori, pagina alarum gosticarun abidius marmorala lunulaque alasum anticarum arcu albo divisa. 


\section{GENUS COLIAS, Boisd.}

Coliades, Auct., Eurymus, Swains.

Chrysalides rectie, medio giblosix. - Antenna breves, rect $x$, in clavam ob-conicam abeuntes. $\Lambda$ la rotunda, flava vel fulva, puncto centrali subtus sub-metallico, in posticis evidentiore.

$+\Lambda l x$ maris posticx ad margin. externum sacculo pulveris glandulosi præditæ.

36 - Thros, Ménétriés. ....... Caucasus.

37 Mrrmidone, Esp., II, O., B. ..... Hungar. . . $\left\{\begin{array}{l}\text { Maii. } \\ \text { Augusti. }\end{array}\right.$

$38\left\{\begin{array}{l}\text { Eousa, Lin., etc. ............ Europ. } \quad \text { Maii. } \\ \text { Var. }\end{array}\right.$

Var. \& Helice, HI., (in Europ. aust.

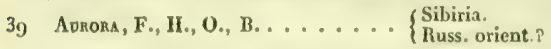

†t Alx maris postica sacculo glandul. destitutx.

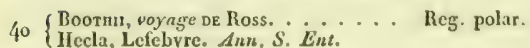

41 Cirnysothene, Esp., II., 0., B., G. . IIungar. . . Augusti.

$42\left\{\begin{array}{l}\text { Neruene, Fisch., B. Species...... } \\ \text { Chrysodona (1), Kinderm. }\end{array}\right.$

$43\left\{\begin{array}{l}\text { Pelide, B., D. . . . . . . . Reg. polar. } \\ \text { An seq. variet } 8\end{array}\right.$

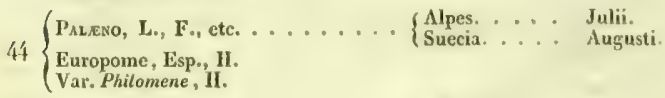

45 \{ Nastes, B., D. .......... Reg. polar.

46 Puconone, Esp., H., O., B..... Alpes. . . . \{ $\begin{aligned} & \text { Augusti. } \\ & \text { Julii. }\end{aligned}$

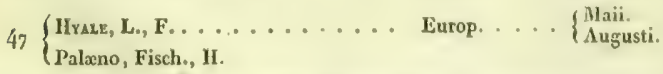

(1) Ifwe concinos varietas e Taganrok differt a $\boldsymbol{C}$. Neriene vera, colore paginxe superioris in utroque sexu luteo-fulvo fere ul apud Edusam, sud characteribus cateris non sccerni potest a Neriene sulphurea. 


\section{TRIBUS LYCANIDES.}

Jarve tenuissime pubescentes, onisciformes, capite parvo, pedibus obsoletioribus. Chrysalides contracte, breves, obtusa, segmentis haud mobilibus. - Palpi distincte tri-articulati. Ala posticx abulomen subtus canali excipientes; areola discoidali aperta, vel nervula spuria obsolctissime clausa. Pedes sex gressorii. Statura parva, gracilis.

1. GENUS THECLA, F., Boist. Polyommati, Lat.; God., Lycana, Ochs.

Chrysalides pubescentes. - Intennæ apicem versus, sensim et modice incrassatx; clava elongata, tenui, cylindrico-ovali. Palpi squammati. Oculi hirti. $\Lambda$ la postica sapius caudata, subtus in nostratibus striga obliqua, dentata, pallida scriptx.

48 Beture, L., etc.......... Europ..... Augusti.

49 Presi, L., etc. . . . . . . . . . . . Junii.

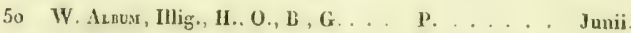

5 I Acaciє, F., H., O., B. ....... \{ $\begin{aligned} & \text { Aurel, ager. Junii. } \\ & \text { Austria }\end{aligned}$

5.2 Esculr, II., O., G., B. . . . . . . Gall. mer. . Junii. $53\left\{\begin{array}{l}\text { Lrwceus, F. G. B.......... Europ. . . . Junii } \\ \text { Ilicis, H., O. } \\ \text { Var. Cerri, H. }\end{array}\right.$ $54\left\{\begin{array}{l}\text { Sprs, F., H., U., G., B. . . . . . Gall. mer. . Julii. } \\ \text { Lynceus, Esp. } \\ \text { Var. Lynceus, If. }\end{array}\right.$

55 (Quencus, L., ete . . ....... Europ. . . . Junii. Var. II., 621 (punctis tribus rubris).

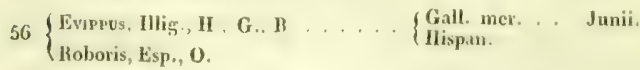

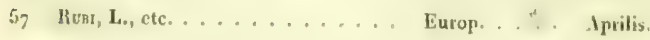


2. GENUS POLYUMMATUS, Boisd.

Polyommati, Lat., God., Lycence, Ochs.

Antennarum clava brevior, crassior. Ala postica ad angulum analem nomihil producta, colore sapius supra fulvo-aureo, in uno sexu saltem nigro punctatx; subtus punctis ocellarilus numerosis.

58 Baulvs, F., H., O., B. ....... G Galloprov. . Martis.

59 Prueas, L., etc. . . . . . . . Europ. .... Aprilis.

$60\left\{\begin{array}{l}\text { Otrowasus, Lefebvre, B., Tr. .... } \\ \text { Legeri, Fr. }\end{array}\right.$

Augusti.

6i Virgadre, L, etc. ......... Mlpes Eutop. . Julii.

G. ILppotroe, L., H., etc......... Gall. occ, ctor. Julii.

$62\left\{\begin{array}{l}\text { Var. Dispar, IIaworth, Curt. . . . Anglia. } \\ \text { Ihippothoe, H. 966. }\end{array}\right.$

$63\left\{\begin{array}{l}\text { Eunvice, II., G.,B. (An var. sequent. P). Alpes Helv. . Julii. } \\ \text { Lurybia, Och. }\end{array}\right.$

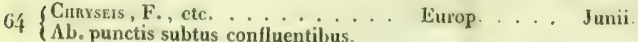

$65\left\{\begin{array}{l}\text { Hiene, F., G., B. . . . . . . . Gall. orient. Julii. } \\ \text { Lampetie, H. }\end{array}\right.$ Ilipponoe, 0.

66 Gondtes, Esp., H., O., B. . . . . . Gall. mer. . Julii.

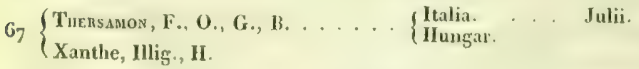

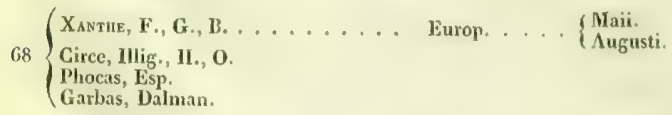

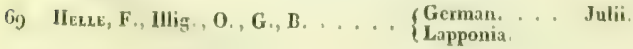




\section{GEIVUS LYCAENA, Boisd}

Polyommati, Lat., God., Lycana, Ochs.

Antennarum clava brevior, distinctior. $\mathrm{Al} x$ rotundat $x$, tenuiores. Color sxpius supra ceruleus, rarius obscure fuscus; sul,tus ciuereo-canescens punctis ocellaribus, numerosis.

+ Al $x$ postic $x$ cauda gracili instructx, subtus strigoso-catenulatx, ad angulum ani punctis ocellaribus auro pupillatis.. G. Lycana, nob. olim.

zo $\left\{\begin{array}{l}\text { Boerica, L., etc. } \\ \text { Colutex, Rossi. }\end{array}\right.$

P...... \{ $\begin{aligned} & \text { Augusti. } \\ & \text { Septemb. }\end{aligned}$

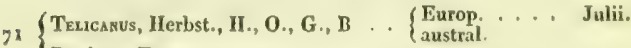
(Bøticus, Esp.

tf $\Lambda$ lac sub-robustiores, subtus punctis ocellaribus sparsis ad margines evidentiores... G. Argus. nob. olim.
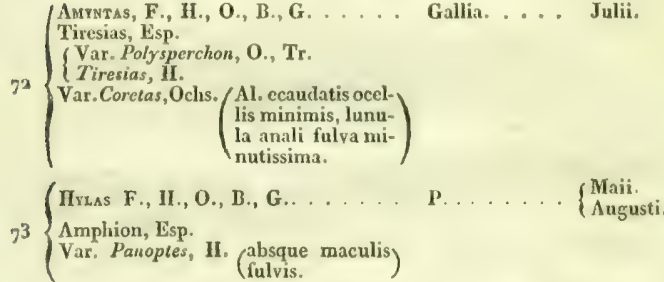

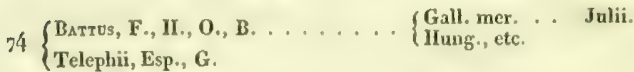

$7^{5}$ Bavius (x), Eversm. ....... Baskiria.

;6 $\left\{\begin{array}{l}\text { NGon, Bork., II., O., G., D. . . . E Europ. .... } \\ \text { Alsus, Esp. }\end{array}\right.$

(1) Species eximia, subtus affinis $L$. Battus, at plane distincta. Ale maris coerulex, (femina fuscar) margine nigro, funbria allo intersecta; posticis punctis marginalibus nigris luaulisque analibus fulvis. Subtus discrepat, primo ibtuitu, a $L$. Baltus, panctis marginalibus uni-seriatis. - Circa Taganroh et etiatu in Turcia a DD. Kindermaun capta. 
$77\left\{\begin{array}{l}\text { Argus, J., ctc. .......... Europ..... }\left\{\begin{array}{l}\text { Junii. } \\ \text { Augusti. }\end{array}\right. \\ \begin{array}{l}\text { alliopis, B., Icones. } \\ \text { Argyrognomon, Bork. }\end{array}\end{array}\right.$

$78\left\{\begin{array}{l}\text { Optilete, F., Esp., O., G., B. . . . . } \\ \text { Var. Cyparissus, II. }\end{array}\right.$

79 Eumedon, Esp., H., O., G. . . . . Alpes, etc. . Julii.

80 Ioss, Ramb., Faun., And. . . . . Ilispan. m. . Junii.

8 I Artaxerces, F., G., B., Tr., H. . . Scotia. .... Julii.

$82\left\{\begin{array}{l}\text { Agestrs, Esp., H., O., B, G. . . . Europ. .... } \\ \text { Var. Allous, H. }\end{array}\right.$

83 prLAon, Fisch............ Sarepta.

84 Rrrunos (1), Eversm......... Russ. m.

85 AQuiro, B., D. . . . . . . . Reg. polar.

(Oraruzos, Esp., O., G., B. ..... Alpes. . ... Julii.

86 Meleager, Illig., $\mathbf{H}$.

$\left\{\begin{array}{l}\text { Var. Pyrenaica. (ocell, posticar, obso- } \\ \text { letis cæcis. }\end{array}\right.$ \{yren. .... Julii.

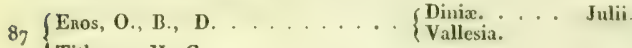

Tithonus, $\mathbf{H}$, G.

88 Antenos (2), Kinderm. . . . . . . Russ. m.

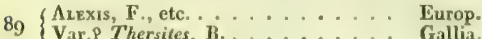

90 \{ Escher, H., B., Tr., D. . . . . Gall. m. . . Julii.

$9^{\circ}$ A Agestor, G., Eucycl.

91 Ilesperica, Ramb., Faun, And... Sierra nevada.

(1) Statura L. Donzelit. Ale in utroque sexu utrinque fuscer, supra iminaculata, subtus dilutiores, punctis niyeis tri-seriatis quorum marginalibus punctulo nigro adjectis. Species singularis nulli allinis, circa Togaorok reperla a filiis D, Kindermann Budensis.

(2) Alis maris nitidis, caernleis, anticis puncto discoidali, fosticis punctis marginalibus nigris; subtus cinereis, punctis ocellatis nigris, maculisqne marginalibus fulvis. Magnifudo Lyecna Eros, color pallidior. 
92 v.Zephyrus, Kinderm. ....... Russ. m.

Specimen or lustratum in collectione D. Contaminc, nimis afine videtur Hesperica D. Ramburi; sed quid certe dicendum de specimine unico!

$$
9^{3}\left\{\begin{array}{l}
\text { Icaruos, Esp., O., B., D. . . . . } \\
\text { Amandus, H. } \\
\text { Agathon, G., Encycl. }
\end{array}\right.
$$

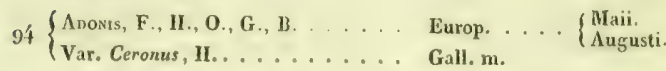

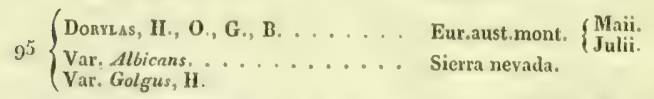

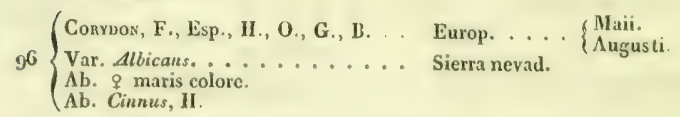$$
97\left\{\begin{array}{l}
\text { Meleacen, Esp., F., G., B. . . . . } \\
\text { Daphnis, H., O., Tr. }
\end{array}\right.
$$$$
9^{8}\left\{\begin{array}{l}
\text { Preretzs, O., G., B. . . . . . . } \\
\text { Atys, II. }
\end{array}\right.
$$$$
\text { 99. Lysumon, H., O., G., B. . . . . . M Mispan. }
$$$$
100\left\{\begin{array}{l}
\text { Acts, W. V., O., G., B. . . . . Europ. . . . } \\
\text { Argiolus, H. }
\end{array}\right.
$$$$
101\left\{\begin{array}{l}
\text { Sezrus, B., Tr., H. . . . . . } \\
\text { Saportx, Dup. }
\end{array}\right.
$$

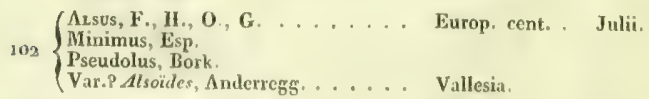$$
103 \text { Donzelı, B., D., Tr., H. .... \{ l Jinia..... Julii. }
$$$$
104 \text { Anmerus, Esp., H., O., G., B. . . . Ilungar. ... Junii. }
$$

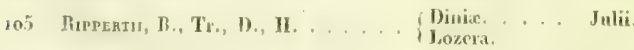


so6 Darron, F., II , etc. ......... Mlpes...... Julii

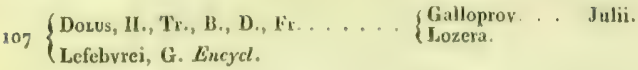

108 Epidolus, An preced. var. 8 (I) . . Turcia.

Menalcas, Kinderm.

$\operatorname{109}\left\{\begin{array}{l}\text { Angrouss, L., O., G., B. ..... P...... } \\ \text { Acis, Illig., II. }\end{array}\right.$

SMelanops, B., Tr., Fr. ...... Krillopror. . Maii.

$110\left\{\begin{array}{l}\text { Saportæ, } 11 . \\ \text { Var. }\end{array}\right.$

Var. Marchandii B., Revue Silb. Catalaunia.

11 I $\left\{\begin{array}{l}\text { Crllarus, F., Bork., Esp., O., G., B Europ. .... } \\ \text { Damatas, H. }\end{array}\right.$

112 Ious, II., O., G., B., D. .... $\begin{aligned} & \text { IIungar. } \\ & \text { Galloprov. . Julii. } \\ & \text { Italia. }\end{aligned}$

113 Arcon (2), F, H., O., G., B., Icones. P....... Julii.

114 Eupremus, 1I, O., B., Icones. . . . \{ $\begin{aligned} & \text { Gall. or. . . J Julii. } \\ & \text { German. }\end{aligned}$

115 Eresus, F., H., O., G., B. . . . . Gall. orient. . Jalii. Arcas., Bork.

ri6 Arson, L., etc. . . . . . . . Loc, arid. Eur. Julii.

(1) Differt tantum a $\boldsymbol{L}$. Dolus Gallica, nervis supra nigris, et pagina inferiore posticarum vitta longitudinali albida, ut in L. Rippertii et Damon, signata, qua vitta in quibusdam $\boldsymbol{L}$. Dolus, revera obsoletissime apparet.

(2) Hane ego speciem in Indice methodico, p. 13, Polyommato Euphemus, tanium varietatem et ex fide Godarti nostri, Gallix meridionalis incolam, dixeram; sed sententia verli debuit; nam anno MDCCCXXX, eirca Chantilly prope P'arisios, reperit Cl. A. Pierret filius, inter entomophilos nobis pregratus, multaque legit specimina. Illam floriferis dumosisque luxuriantis sylva partibus volitantem mibi pro certo afirmavit; larvam Leguminosas; forsan viciam eraccem, pasci putat, sap̧ius enim inaginera is bac planta naseentem et adbuc madidam deprebendit. 


\title{
IV. TRIBUS ERYCINIDES, Boisd.
}

Larva pilosx, pigra, oblongo-clongatx. Chrysalides sxpius pilosula, antice obtuse, plus minusve contractix. - Pedes antici maris spurii, feminx, (paucissinis exceptis), omnes completi. Ala per quietem horizontales, rarissime erectx.

\author{
1. GENUS NEMEOBIUS, Step., Boisd. \\ Hamearis Curtis, Argynnis, Lat., Iycana, Tr., Suppl.
}

Larva onisciformis, pilosula. Chrysalis obtusa, pilosa. - Palpi hirsutissimi, capite breviores, articulo ultimo brevi, obtuso. Antenux abrupte clavate. Pedes maris antici breves, valde pilosi, femina contra longiores, minus pilosi. Alæ per quictem erectx.

Nota : Hocce genus signaturis et pictura pro Mclitare, primo intuitu, facile liabetur.

117 Locms, L. etc. .......... \{ $\begin{aligned} & \text { Europ. cent. . Maii. } \\ & \text { et horeal. }\end{aligned}$ 


\section{PENDULE.}

Chrysalides liber $x$, pendulæ, ano tantum adfixx.

\section{TRIBUS DANAIDES.}

Larvæ glaberima, dorso conjugatim tentaculatx. Chrysalicles deauratx, haud angulose. - Palpi lreves, distincte tri-articalati. Alæ posticx arcola clausa. Pedes quatuor gressorii in utroque sexu.

1. GENUS DANAIS, Boisd.

Danaides, Lat., Euploce, F., Ochs.

Palpi remoti, articulo ultimo brevi, recto, lineari. Capite punctato. Alx sub-repandx, latiores; postice maris ad angulum ani, modo sacculo nigricanti pradita, modo macula uigerrima, nervo albo divisa, signatx.

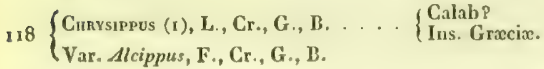

(1) Ex Africa orta, et paucis tantum annis cirea Noppoliun eapta, sed ab abno MDCCCIX non amplius reperta. 


\section{TRIBUS NYMPIIAYIDES.}

Larva dorso spinosin. Chrysalides angulosx. - Pedes quatuor gressorii

\section{GENUS LIMENITIS, Boisd. \\ Limenites, Och., Nymphales, Lat.}

Larve spinis inxqualibus armata. Chrysalides anritx, dorso prominentes, metallice maculatæ. - Antennæ corporis longitudine; clava gracili, haud determinata. Palpi pilosi, capite vix lonģores. Ala den ticulatæ, in nostratibus fusca, albo maculatx.

$$
\text { + Alæ oblongæ. G. Noptis, Fab. }
$$

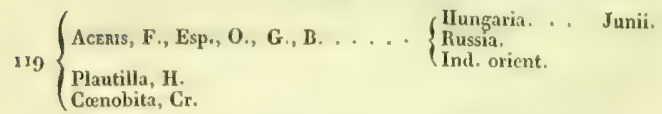
$120\left\{\begin{array}{l}\text { Lucıцı, F., H., O., G., B. ...... } \\ \text { Camilla, Esp., Borkh. }\end{array}\right.$ + $\Lambda$ l x trigonæ.

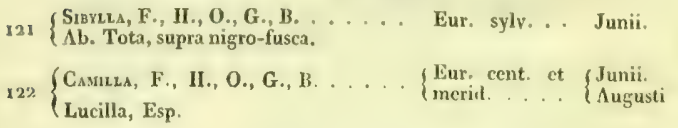

2. GENUS NYMPIIALIS, Boisd.

Limenitis, Och., Nymphalis, Lat. God.

Larva dorso tuberculato, sub-spinosæ, Populos et Salices pascentes. Chrysalides antice bifidx, dorso valde prominentes, absque maculis metallicis. - Antenna corporis longitudine, clava haud determinata. Palpi brevius pilosi, linguam non occultantes; articulomedio longo, ultimo brevissimo, pilis occultato. Alx dentat $x$. Statura magua. 
123 $\left.\begin{array}{l}\text { Popur, L. etc. } \\ \text { Var. Tremulre, Guénée (absq macu-) } \\ \text { lis albis. }\end{array}\right)$

\section{GENUS ARGYNNIS, Ochs., Boisd. Argyunes, Lat.}

Larva ramoso-spinosx; collo armato. Chrysalides augulato-nodulosx, metallice maculatx. - Antennx capitulo brevi, ovato, compresso, sub-patelliformi. Palpi capite longiores, squamato-lirsuti, apice distantes; articulo medio magno, ultimo minuto. $A 1 x$ suh-denticulata, supra fulva maculis strigisque nigris; subtus postica maculis strigisve margaritaceis plus minusve ornatx.

\section{+ Majores.}

124 Pandora, Esp., O., H., B....... Europ, austral. Junii.

$\left\{\begin{array}{l}\text { Cynara, } \\ \text { Maja, Cr. }\end{array}\right.$

$125\{$ Papни, L., pte.

V Var. Y Valezina, Esp.

Europ. . . . Julii

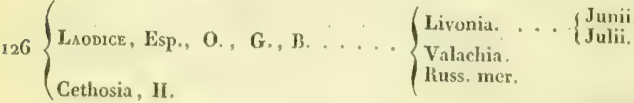

327 Alexandra, Ménétriés. . . . . . Caucasus.

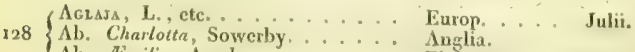

Ab. Emilin, Acerby. ........ Finland.

$129\left\{\begin{array}{l}\text { Crnese, Bonelli, If., Tr., B. . . } \\ \text { Elysa, G., D. }\end{array}\right.$

Adippe, Fab., etc. ........ Furop. . . Julii,

13o $\{$ Var. Sheodoin, Iinp.

Var. Chlorodippe. ......... S Sierra neyada.

Sicilial.

$131\left\{\begin{array}{l}\text { Nıore, L., etc. } \\ \text { Var. Aglaope } \\ \text { Walner. . . . . . Reg. sub-Alp.. Julii. }\end{array}\right.$

(11). Eris, Sclimulierr. 
${ }_{132}\left\{\begin{array}{l}\text { Lathonıs, I., ete. . . . . . . Furop. . . . . } \\ \text { Ab. (Alis posticis subtus totis argentatis). }\end{array}\right.$

${ }_{33}$ Polatis, B., D. .......... $\left\{\begin{array}{l}\text { Alpes Norveg. } \\ \text { Cap Nord }\end{array}\right.$

.34 Freusa, Th., H., O., B., G. ..... Lapponia. . J Juhi.

135 Arathusia, F., Esp., O., G., B. .. Alpes. .... Julii.

36 Curariclea, IIerb., O., H., IJ. .... Alpes Norreg. Julii.

$₫ 36$ Var. Boisduvalii, Sommer, D. B. . Reg. polar.

337 FrJGG , Th., O., 11., G., B..... Lapponia.

33 Dapise, F., 1I., O., G., B. . . . . Reg sub-Alp. Junil.

139 Trone, H., O., G., B. . . ..... Itelvet..... Junii

139 varietas multo dilutior. . . . . Lapponia.

$240\left\{\begin{array}{l}\text { Iso, Esp., O., G., B., Fr. ...... } \\ \text { Dictynna, H. }\end{array}\right.$

141 Hecate, F., H., O., G., B. .... \{ $\begin{aligned} & \text { Galloprov. } \\ & \text { German, etc. Junii. }\end{aligned}$

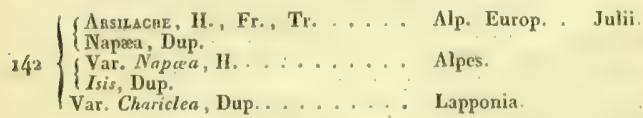

$143\left\{\begin{array}{l}\text { Pales, F., O., H., Tr., B..... } \\ \text { Yar. Isis, H. (non Dup.) } \\ \text { Ab. Balamelas, Bugnon. }\end{array}\right.$

144 Dis, L., etc. ........... Europ. sylv. . $\left\{\begin{array}{l}\text { Mlaii. } \\ \text { Julii. }\end{array}\right.$

145 Euphosise, L. etc. . . . . . E Europ. sylv. I Maii.

$\$ 46$ v. Selenis, Eversm., Lefelovre.. . . . Russ, or 


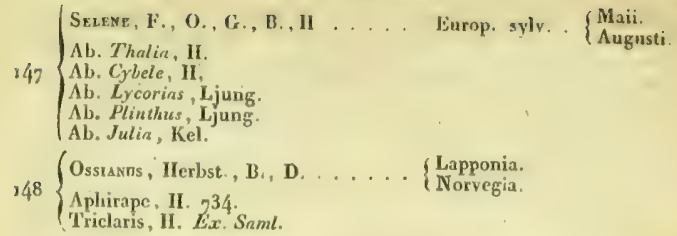

149 Apmase, II. 23, O., B., G. . . \{ $\begin{aligned} & \text { Suecia. } \\ & \text { German, } \\ & \text { Belgia. }\end{aligned}$

\section{GENUS MELITAA, Fab., Ochs,, Boisd. Argynnes, Lat., God.}

Larva spurie spinosx, tuberculis carnosis pubescentilus, spinas mentientibus, instructx. Clirysalides vix angulatx, ad summum dorsi punctis elevatis taberculata.-Intennx capitulo pyriformi munita. Palpi rapite longiores, apici distantes, tenues; articulo medio lirsuto, ultimo dimidio loreviore, acuminato. Pedes antici feminx longiores, sub-nudi. Statura media.

$+$

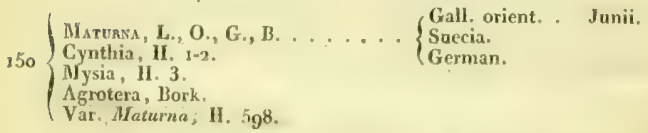

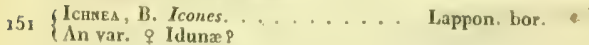

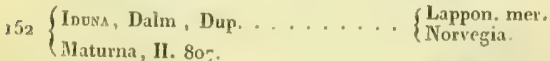

${ }_{153}\left\{\begin{array}{l}\text { Crnsma, F., O., G., B. . . . . . . . } \\ \text { Trivia, Esp. }\end{array}\right.$

Cynthia, H. 608,569.

Mysia, H. $939-946$.

154 Merore (1), Deprun., B., D. Fr., Tr, Helvetia....Janii.

(1) Larvam hujus specieb invenit Dorn. Anderreg5, circa Gamsen; accedit valde ad

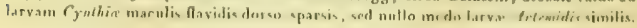


Antemis, F., H., O., G., B. . . . Europ. .... Maii.

Maturna, Esp.

Var. Desfontainesi. Maxima, col. vivaciori, punctis marg. flavo-pallidis. Hispan mer.

\section{tt}

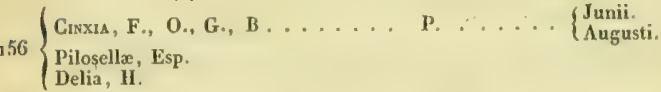

137 Rhodopensis, Frivaldski (1), Fr. : ... Turcia. .

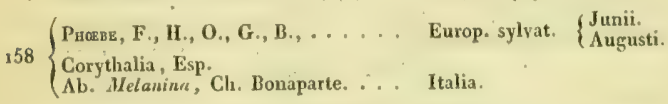

I59 v.Atmeria, H., Dup., an var. præced P. Russ. mer.

160 . Arduna, Esp.; God. . . . . . . Russ. mer.

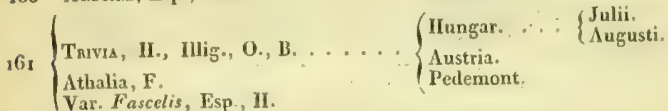

$362\left\{\begin{array}{l}\text { Didrns , F., O., G., B. . . . . . . Europ. cent. . Junii. } \\ \text { Cinxia, II. }\end{array}\right.$

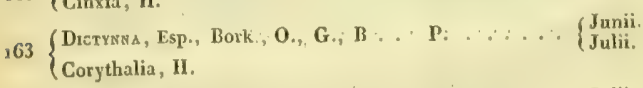

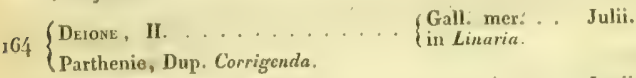

Partheme, Bork., O., B., G. .... Europ. sylvat. (Junii.

Athalía minor, Esp.

Dictynna, Thunb.

Var. P Asteria, Fr., Tr. (3)..... Helvetia.

(1) Afinis certe Cinxis, at multo major, sæpius fhabem superans : discrejat a Cinxia, fascia media albida quo extus lineis duabus nigris a fascia fulva punctata, spporatur, at ejusdem fascia albida punctis nigris, in medio locatis: Cuteris characteribus Cinsia simillima.

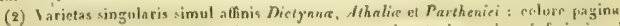
superioris cum Dictynna congruit, tatura pero et signaturis pagina inferioris ad Pnrthenieno proprivs accedit. 
Artal1a, Bork., Esp., O., G., B. . . Europ. .... . Junii.

Maturna, F., H.

Ab. Pyronia, $\mathrm{H}$.

Ab. Iphaea, II.

Ab. Cimothoe, Bertolini.

Ab. Mertha, Quensel, Dalm. ..... P

Ab. Fulla, Quensel, Dalm......... Suecia.

\section{GENUS VAINESSA, Osch., Boisd.}

Larvæ ramoso-spinosa, segmento primo anoque muticis. Chrysalides angulatx, antice bi-mucronatx, dorso dentat $x$, muculis aureis ornat $x$. - Antenna rigida capitulo oblongo apice albido. Palpi capite dimidio Iongiores, piloso-hirti et squamati; articulo medio duplo longiore, ultimo conico. Oculi hirti. Pedes antici valde hirti. Ala angulatx, anteriores saltem, eximie depictæ. Statura sat magna.

$+$

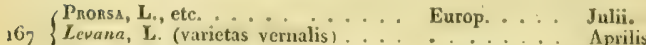

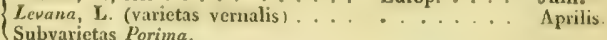

t† G. Cyuthia, F.

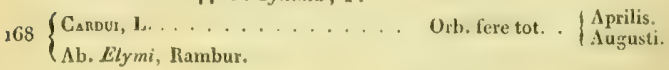

169 Aralanta, L., etc. . . . . . . E. Europ. .... Peran.

t+t

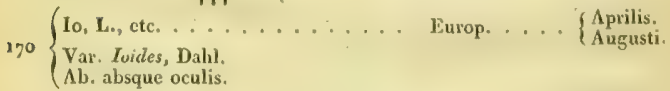

171 Antiopa, L., etc. ............. Europ. ... $\begin{aligned} & \text { Aprilis. } \\ & \text { Augusti. }\end{aligned}$

$t+t+$

i 2 Untice, L., etc........... Europ. .... Peran.

$17^{3}$ Icrivuss, Bonelli, B., D., Tr. . . . . Sardinia. . . Fistate.

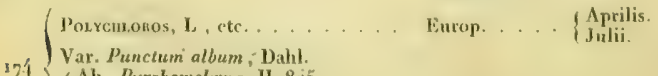

\{ Ab. Pyrrhomelenn, H. 8.j.

T'estuilo, Esp.

IIybr. \& Xanthochloros. 
'7j Xantuonelas, Esp., H., O., G., B.. German. . . $\left\{\begin{array}{l}\text { Julii. } \\ \text { Augusti. }\end{array}\right.$

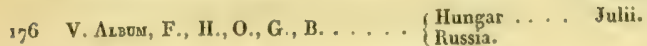

\section{ttt+t}

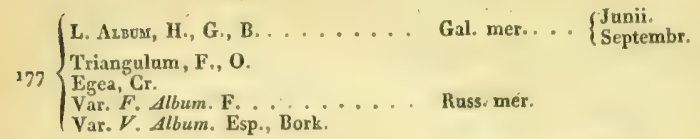

${ }_{178}\left\{\begin{array}{l}\text { C. Albur, L., etc. . . . . . . . Europ. . . . } \\ \text { Ab. A. (maculis magnis nigris effusis), }\end{array}\right.$ 


\section{TRIBUS LIBYTIIEIDES.}

Larve inermes, elongatie, pubescentes, eapito rotundato. Chrysalides dorso carinate. - Mares tetrapodi, feminx vero hexapodx. Ale angulatx.

\section{GEYUS LIBYTHEA, Latr., Boisd. \\ Hecaerge, Ochs.}

Larva cognitæ, arbores generis $C$ cltis pascentes. - Palpi conniventes, squamoso hirsuti, horizontales, in rostrum longum producti.

179 Certis, F., Esp., O., G., B. .... $\begin{aligned} & \text { Gall, mer } \\ & \text { Italia. } \\ & \text { Hispan., etc. }\end{aligned}$ 


\section{TRIBUS APATURIDES.}

Larvx inermes, postice scusim attenuata, capite tantum spinoso, ano attenuatæ. Chrysalides sub-ingulato-compresse vel angulato rotundatx. Pedes quatuor gressorii. Areola alarum posticarum aperta. Volatus nobilis, velificans.

\section{GENUS ChARAXES, Ochs., Boisd.}

Nymphales, Latr.

Larw $x$ capite quadricorni, in caudam depressam desinentes. Clurysalides abbreviatx, rotundato-sub-conicx, dorso vix carinatx. - Antennx in clayam fusiformen sensim abeuntes. Palpi sub-distantes, apice subcomirentes, capite longiores, piloso-squamati; articulo ultimo brevi olstuso, sub-nudo. Alæe posticæ crecx, denticulatæ, sæpius caudatæ. Statura robusta, magna.

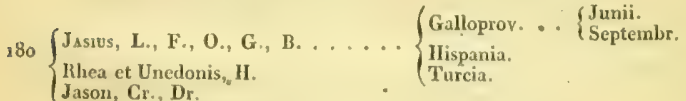

2. GENUS APATURA, Ochs., Boisd.

Nymphales, Latr.

Larva capite bi-spinoso, ano bi-mucronato. Chrysalides lateraliter compressæ, dorso carinato, capite bifido.-Intenna in clavam fusiformem sensim crescentes. Palpi conniventes, basi sub-distantes, capite longiores, piloso-squamati; articulo ultimo acuto, sub-inflexo. Alre posticx subtus ad angulum ani ocellatx. Statura major, robustior.

i $81\left\{\begin{array}{l}\text { Ius, } \mathbf{L}_{.,} \text {etc.. } \\ \text { Ab. Beroe, F, Herbst. } \\ \text { Tole, II. }\end{array}\right.$

Ilia, F., H., O., G., B. . . . . . . . P., etc. . . . Jınii ${ }_{882}\left\{\begin{array}{l}\text { Var. Clylie, H. } \\ \text { Yisis Liter, Borkh. } \\ \text { Yar. }\end{array}\right.$

P., etc.

$282\{$ Var. Astesita, H. 812

Var. Iris rubescens, Rossi.

Var. Iris metis, Kinderm. . . . . . Smyrnia. 


\section{IX.- TRIBUS SATYRIDES.}

Larva graminivore, postice attenuatx, bi-mucronatx, muticx. Chrysalides sulb-giblosae, sub-muticx, depauperata.-Palpi sat producti, hirti. Ala ocellata; areola discoiddalis posticarum clausa. P'edes quatuor gressorii,-Volatus subsultans, humilior.

\section{GENUS ARGE, Esp., Boisd. Satyri, Lat, Hipparchice, Ochs.}

Chrysalides pallidx, capite nigro bi-notato, humi sub graminibus sxpius jacentes, - Antennæ longiores, clava haud determinata, basi evanescente, Palpi remoti, pilis rigidis, rariusculis fimbriati, articula ultimo valde distincto, breviter ciliato, apice nudo. Alx albx, nigro maculatim strigatæ; anticarum nervo costali tantum, modice ad basin, dilatato.

\section{$+$}

\{ Incuests, II., O., G., B. . . . . Occitania. . J Junii.

\{ Arge Nemạusiaca, Esp.

184 Hruata, Ménétriés, B. . . . . . Caucasus.

(Galatula, L., etc. ........ Ėurop. . . . Junii. $185\left\{\begin{array}{l}\text { Var. Procida, } \\ \text { Galaxera, Esp. }\end{array}\right.$

Var. Galene, $0 \ldots \ldots . . . . .$. Italia.

Var. Turcica (fere tota nigra). .... Turcia.

Ab. Leucomelas, H., Esp. ....... Alpes.

$+t$

Arge, F., G.

, B., D. ...... Illungar.

Junii.

Arge Tiussix, Esp.

Suwarovius, llerbst.

, Var. Alropos, H.

\{ Lyssianassa, Dahl.

18 Cleantrie, 13. 11. (an var, pracedent. 8). Dinia..... Junii.

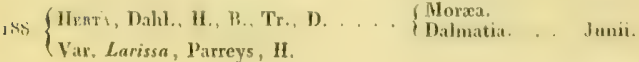


$189\left\{\begin{array}{l}\text { Tirta, Klug. . . . . . . . . } \\ \text { Darceti, Lefebvre, D. }\end{array}\right.$

igo Teneates, Ménétriés, B. . . . . Ca Cacasus.

ig1 $\left\{\begin{array}{l}\text { Psrcue, H., G., B. . . . . . . . } \\ \text { Syllius, Herbst., O. } \\ \text { Arge Occitanica, Esp. } \\ \text { Ab. Ixora (oculis nullis). }\end{array}\right.$

192 Phenusa, Dahl (an sequent. var. Joc. 8 ). Sicilia. . . . Junii.

$19^{3}\left\{\begin{array}{l}\text { Ampurmat, Jl., D., G., B. . . . . Calabria. . . Junii. } \\ \text { Arge, O., Tr. } \\ \text { Arge Sicula, Esp. }\end{array}\right.$

$194\left\{\begin{array}{l}\text { Ises, Hoffmansegg, O., B. . . . . Hispan mer. } \\ \text { Thetis, II. }\end{array}\right.$

\section{GEIVUS EREBI $\Lambda$, Hoisd.}

Erebice, Dalm. Hipparchio, Uchs. Satyri, Lat.

Antenux clava ovali, oblonga, compressa, preditx. Palpi sub-remoti, pilis densis, setosis instructi; articulo ultimo vix distincto, villoso. Alx uigrx, fulvo ocellatx; anticarum nervis haud basi dilatatis, vel costali tantum in paucis, aliis crassiori.

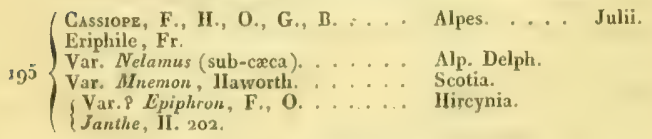

${ }_{19} 6^{\circ}$ Anete, F., H., O. (an sp. distinct. P). Alp. Austr.

197 Pharte, Esp., H., G. (anvar. sequent.8). Alpes. .... Julii.

$19^{8}$ \{ Mecampos, Esp., O., G., B. . . . A Alp. Helv, . J Julii.

199 Mestra, Esp., H., O., G., B. ... Alp. Helv. . Julii 


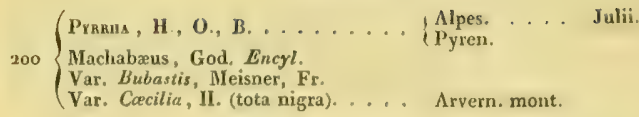

sus OFme, H., O., G., B. ........ Alpes. .... Julii.

20׳2 CEto, II., O., G., B. . . . . . Alp. Helv. . Julii.

$203\left\{\begin{array}{l}\text { Psodea, O., II., G., B....... } \begin{array}{l}\text { Styria. } \\ \text { Hungaria. }\end{array} \\ \text { Var. Eumenis, Dahl, Fr. }\end{array}\right.$

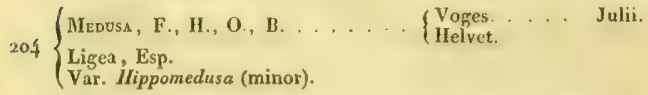

205 Strgne, O., G.. B. . . . . . Reg. sub.Alp. Julii.

$206\left\{\begin{array}{l}\text { Evis, God., Lefeb., B., Tr. .... } \begin{array}{l}\text { Pyren. } \\ \text { Alp. Helv. }\end{array} \\ \text { Bonellii, H., Fr. }\end{array}\right.$

207 Epistrenz, B., Tr., D. . . . . . Dinia. . Martis.

Arra, F., G., B. . . . . . . $\begin{aligned} & \text { Caucasus..... Junii. } \\ & \text { Dalmatia. }\end{aligned}$

208 Afer, Esp., 0.

Phegea, H., D.

Var, \& Dalmatn, G. Encyl.

$200\{$ Méras, Herbst., O., B. . . . . . Alp. Ilung. . Julii.

$210\left\{\begin{array}{l}\text { Lefebvrex, Blecto, God. Pap, de France. } \\ \text { Alo }\end{array}\right.$

211 Nerase, Tr., B, D., Fr. . . . . A A . Carinth. . Augusti.

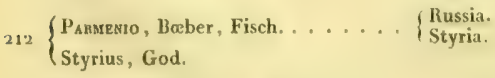

213 Scipıo, B., D.. Guén., H.-Gey. .... Alp. Provinc. Julii. Alecto, H P 515. 


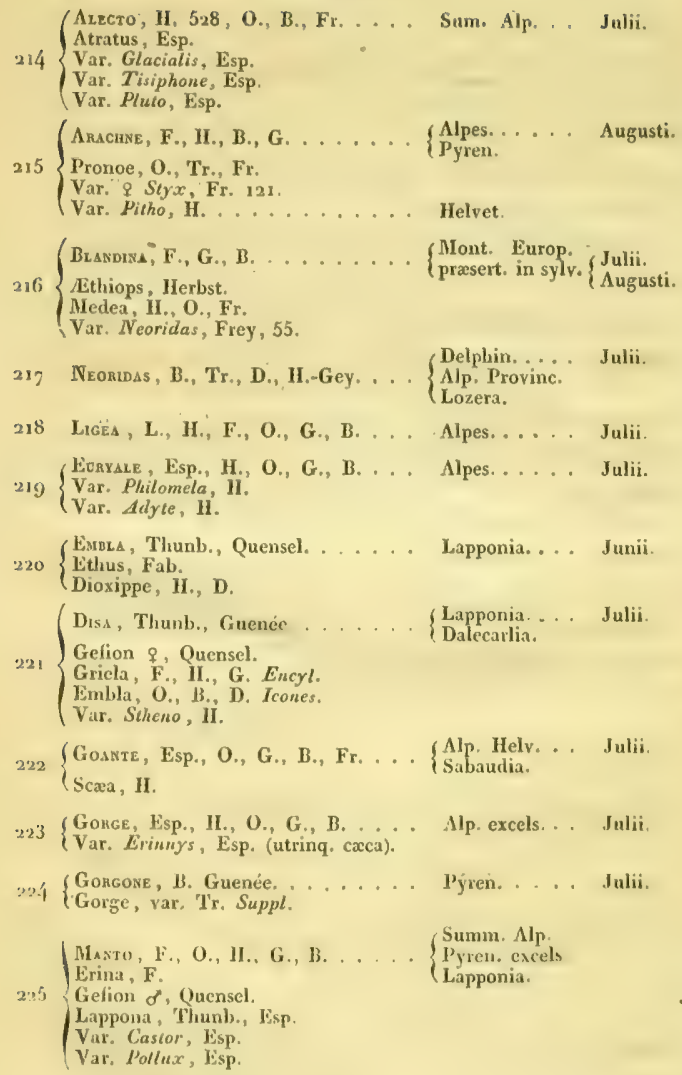




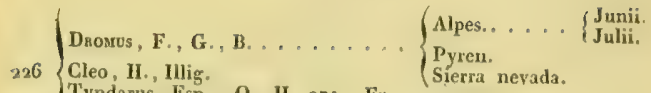

T'yndarus, Esp., O., H. 971 . , Fr.

Var. Cassioides, Esp......... Hungaria.

Neleus, Fr.

\section{GEIVUS CHIONOBAS, Boisd, Dup.}

Satyri, Lat. Hipparchice, Och. Erebiae, Dalm.

Antennx a basi ad apicem sensim clavatx. Palpi sub-remoti, pilis rariusculis fimbriati; articulo ultimo brevi, distincto, villoso. Al $x$ livide fulvæ, vel cinereo-lutescentes; anticarum nervo costali longe, sed modice, dilatato, nervo medio vix aliis crassiori.

$227\left\{\begin{array}{l}\text { Aeuro, Esp., H., O., G., B. .... } \\ \text { Norna, H. I4ı. }\end{array}\right.$ $228\left\{\begin{array}{l}\text { Nonns, Th. Esp., O., G., H., 63, B. \{ Lapponia.... Julii. } \\ \text { Var. Celano, H. } \\ \text { Var. Hildr, Quensel. }\end{array}\right.$ 299v. $\left\{\begin{array}{l}\text { Tarpeia, F., Esp., D., G., B. . . , \{libiria. } \\ \text { Celimené, Cr. }\end{array}\right.$

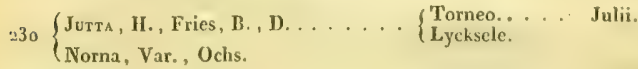
231 BaLdER, B., D. . . . . . . . Reg. polar.

232 Boores, B., D., Tr. . . . . . . Reg. polar. . Julii.

233 Bore, H., O, Dalm., B. . . . . . Lapponia ... Julii. $\left\{\begin{array}{l}\text { Fortunatus, F. } \\ \text { Norna, var. Thumb. }\end{array}\right.$

234 OEno, B. D. . . . . . . . . . Cap Nord.

${ }_{235}$ Asso, B............... Sibir. bor. 


\section{Genus satyrus, Boisd.}

Satyri, Latr. Mipparchice, Ochs.

Antennæ modo capitatx, modo in clavam gracilem abeuntes, modo absque clava determinata. Palpi sub-remoti, pilis rigidiusculis hirti. articulo ultimo brevissimo , conoideo. Ale rotundatix, vel sub-denticulatæ; anticarum nervo costali subito ad basin valde inflato, nervo medio etiam dilatato, nervo radiali modo normali, modo uti aliis dilatato.

+ Nervis duobus dilatatis. Larva pubescentes.:

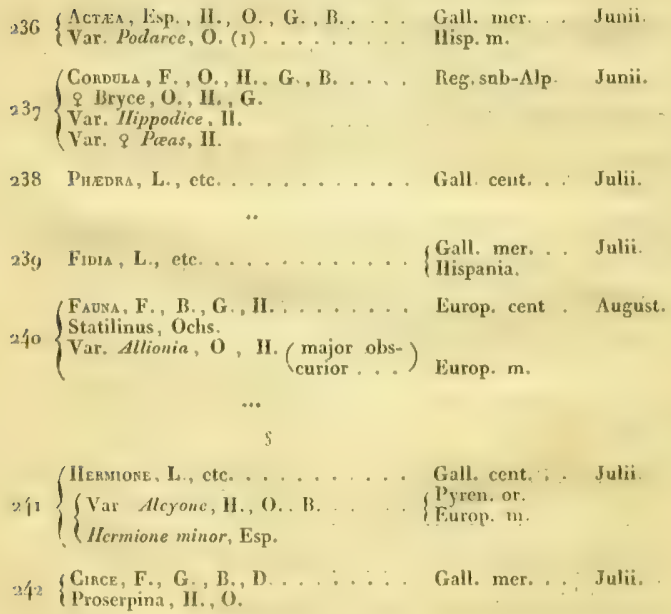

(1) Vidi multa specimina utriascyue sexus, captn in flispania australiovi a $\mathrm{Cl}$. Hambur ;

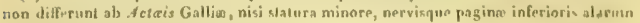
posturam sub-albido-pulverulentis. 


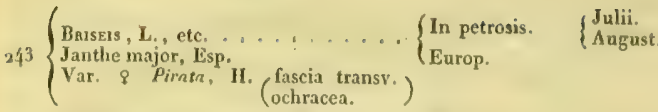

$244\{$ Anтне, Baber, O., G., B. .... liuss. m . . Julii.

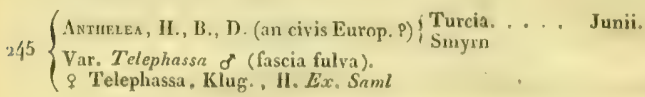

246 Avrosob, F. Esp., H. , B. . . . . Stepp. Rnss... Julii.

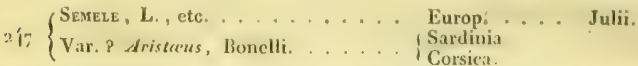

$24^{8}\left\{\begin{array}{l}\text { Hrppozxe, IIerbst., O., B. . . . . } \begin{array}{l}\text { Russ, mer. } \\ \text { Sierra nevad. }\end{array} \\ \text { Agave, H. } \\ \text { Alcyone, F., G. Encycl. }\end{array}\right.$

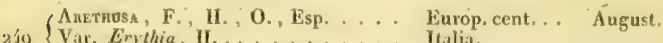

x行 $\left\{\begin{array}{l}\text { Var. Erythia, II... Italia } \\ \text { Var. } 9 \text { Boabdil, Ramb. Andal. Sierra nevada. }\end{array}\right.$

$250\left\{\begin{array}{l}\text { Neonsras, G., B., D. . . . . . } \\ \text { Marmoræe, H. } \\ \text { Jolaus, Bonelli, Tr., Fr. }\end{array}\right.$

25ı Nanics, H., B., D., Gucnée..... \{ $\begin{aligned} & \text { Sibiria. } \\ & \text { Ural. }\end{aligned}$

${ }_{252}$ Eudora, F., Esp., H., O., 13. ... \{ Gall. m. ... Julii.

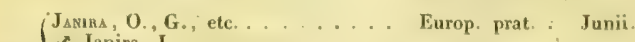

, $53\left\{\begin{array}{l}\text { of Jamira, L. } \\ \text { o Jurtina, } \\ \text { Var, }\end{array}\right.$

( $\begin{aligned} & \text { Jurtina, } \mathbf{L}_{1 .+} \\ & \text { Var, Hispulle, Esp. , 11. . . . . . Eur. austral. }\end{aligned}$

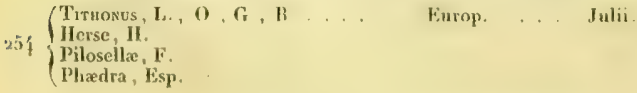


${ }_{25} 5$ Iоя, Esp., П., O., G . . . . . Gall, m. . . Junii.

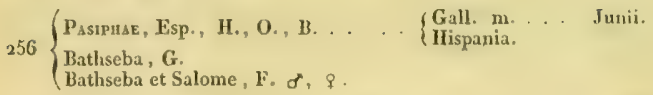
"*e*e Oculi hirti. an gen. propr. ?

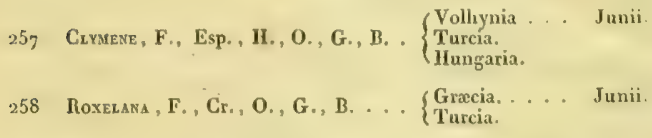

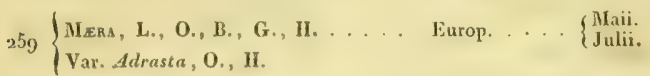

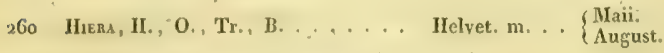

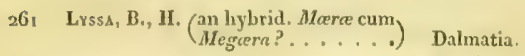

262 MEgrRs, L., etc. . . . . . . Lurop. . . . \{ Maii.

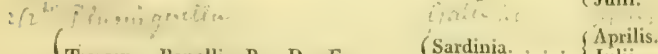

$263\left\{\begin{array}{l}\text { Tigruus, Bonelli, B., D., Fr } \cdots\left\{\begin{array}{l}\text { Sardinia.... } \\ \text { Corsica. }\end{array} \text { Paramegæra }, \text { H. }\right.\end{array}\right.$

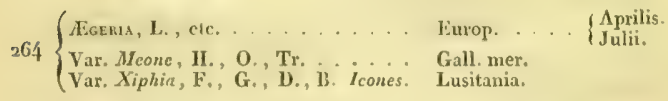

265 Dejaña, L., etc. .......... Europ. cent. . Junii.

\footnotetext{
${ }_{266}\left\{\begin{array}{l}\text { IIrpenantuos', L., etc. . . . . . . Europ. . . . Junii. } \\ \text { Polymeda, H. Huller (absque oculis }\} .\end{array}\right.$

Ab. B. (oculis maximis ovatis).
} 
t+ Nervis tribus dilatatis. Larvix glabru.

- An gen. proprium?

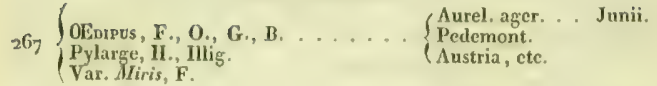

$268\left\{\begin{array}{l}\text { Heno, L., H., O., G., B. . . . . . . } \\ \text { Sabæus, F. }\end{array}\right.$

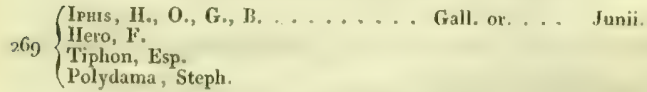

270 Arcakius, $\mathbf{L}_{\text {s. etc. }}$

Europ. .... Junii.

2 I $\left\{\begin{array}{l}\text { Philea, H., G., B. } \\ \text { Satyrion, Esp., O. }\end{array}\right.$

Alp. Helv. . . Julii.

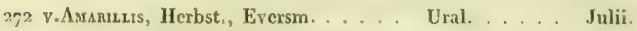

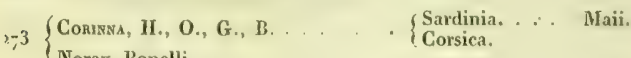

Norax, Bonelli.

$2 \%\left\{\begin{array}{l}\text { Donus, Esp., O., G., B. ...... I Gall. mer... Julii. } \\ \text { Dorion. II , Illig. } \\ \text { Lizetta, Cr. }\end{array}\right.$

$2,5\left\{\begin{array}{l}\text { Leander, F., O., G., B. . . . . . . \{ l lungar. . . Junii. } \\ \text { Clite, H. }\end{array}\right.$

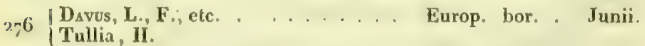

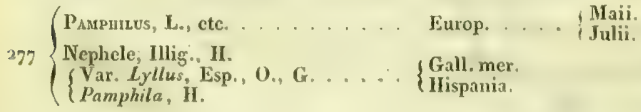

${ }_{2 \eta} S\left\{\begin{array}{l}\text { Prnqne, H., O., B. . . . . . Kuss. mer. . Junii. } \\ \text { Phryneus, F. G. } \\ \text { Tircis, Cr. }\end{array}\right.$ 


\section{IN VOLUT}

Chrysalides ano filisque transversis alligatx, sub tela tenui inter folia quiescentes.

\section{TRIBUS HESPERIDAE (1).}

Larve tortriciformes, inter folia alligata vel contorta degentes. Chrysalides vix sub-angulosa.-Caput magnum, crassum cum oculis thorace sxpe latius. P'edes sex completi. Areola alarum posticarum aperta.

\section{GENUS STEROPES, Boisd. Hesperia, Latr., Ochs.}

Caput thoracis latitudine. Antennx haud uncinata, clava sub-ovata. Palpi remoti, hirsuti, articulo ultimo graciliori, distincto. $\Lambda$ la ommes per quietem erectx, conniventes. $\Lambda$ bdomen alis posticis longius. Statura debilior.

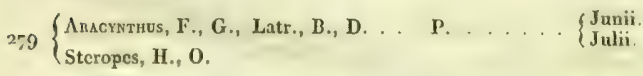

280 P PaNiscos, F, Illig, H, O., G., B. . P. ..... Mäi. Var. Sylvius, H., O., G. ........ Circa Prunswick.

2. GENUS IIESPERIA, Boisd.

Hesperia, Auct.

Chrysalides antice mucronatæ, abdomine vagina libera instructx. Caput crassum, cum oculis thorace latius. Antennx rigida, clava ovata, sxpius mucronulo extus arcuato pradita. Palpi valde hirsuti, articulo ultimo gracili, acuto. Alx anticx per quietem semi-crectx, posticæ patulæ, horizontales. Statura robustior.

(1) Cl. Stephens (Systematic Catal. of British insects) tres Hesperins America septentrionatis (Oileus, Lin. - Bucephalus, Steph. - Vitellius, Fab.), ut hospites anglicas enumerat; sed pro certo nunquam occurruat, nec in Auglia sec io Europa australiori. 


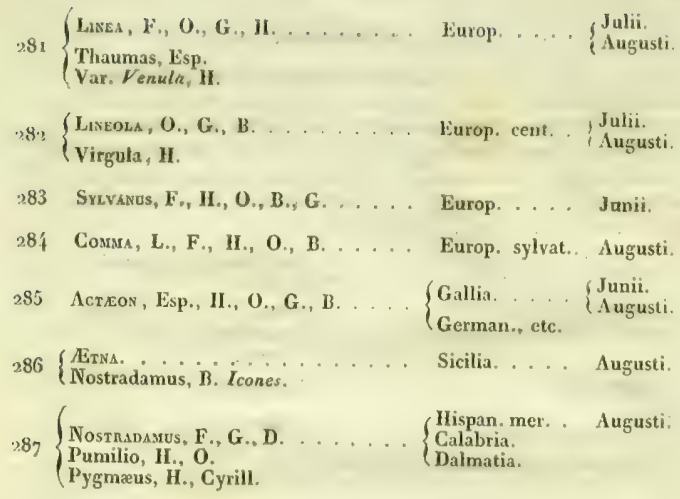

3. GENUS SYRICTHUS, Boisd.

IIesperiae, Auct.

Larvx collo straugulatx Chrysalides conicx.-Caput sat magnum, thoracis vix latudine. Antennarum clava oblongo-ovata, obtusa, extus sub-arcuata. Palpi hirsutissimi, articulo ultimo gracili, distincto. Ala omnes denticulata vel dentatx, per quictem patulx, horizontales (cinereo-fuscx, albo tessellatx, fimbria intersecta); posticis abdomen sub-brevius.

† Alx dentatx. G. Spilothyrus, Dup.

288 Arтhé, H., G., D., Guenée...... Gallia. .... Malii.

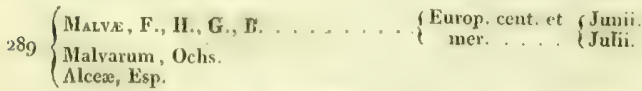

2go $\left\{\begin{array}{l}\text { Lavatere, Esp., H., O., G., B. .... Reg. sub-Alp. Augusti. } \\ \text { Alcex, F. }\end{array}\right.$

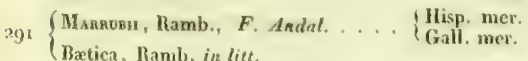


ft Alx denticulatx.

Nota. Species hujus gregis pleraeque valde aflines et sxpissime ah auctoribus confusx, unde synonymia eo magis inextricalilis, quod efligics accuratissima ad illarum cognitionem haud plane suficiunt.

Cl. Doctor Rambur ad species certius secernendas, characteres ex appendicibus genitalibus maris denudatis duxit, et hac ratione plure novas, colore maculisque alarum a specichus dictis Alvcus et Fritillum vix discrepantes, invenit.

292 Proto, O., G., B., H. . . .... Gall. mer. . Julii.

3 Italia..... Junii.

293 Sidx, F., Esp., H., O., G., B. .... $\begin{aligned} & \text { Talloprov. } \\ & \text { Turcia. }\end{aligned}$

294 Crnare, B. (1).......... Taganrok.

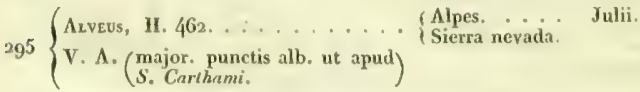

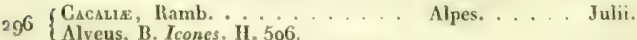

297 v.Tessezusz, O., H., Tr. . . . . . . Russ, mer. $\therefore\left\{\begin{array}{l}\text { Maii. } \\ \text { Augusti }\end{array}\right.$
$298\left\{\begin{array}{l}\text { Cartuasi, O., H., Tr. . . . . . . Europ. cent. . } \\ \text { Tesselum, G., Encycl. }\end{array}\right.$

299 Serrutule, Ramb. (2). . . . . . Sierra nevada.

300 Osopond, Ramb. (2)....... \{ Gall, mer.

301 Crrsir, Ramb. (2)......... P.

302 Canuine, Ramb. (2). . . . . . Alpes.

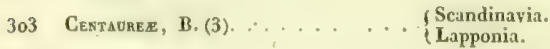

(1) Statura Sida majoris, et ab omnibus distincta magoitudine macnłarum albidarum.

(2) Cum $S$. Fritillum in collectionibus confusa et permixte.

(3) Statura el ales antice fere ut apud S. Fritillum; sed bene distincla nacula costali posticarum alba, prosestimque maculis numerosis concinoe tessellatis nervisque albidis pagine inferioris earumdem alarum. 
304 Fratruma , 11. . . . . . . . Gall. m. ... Augusti.

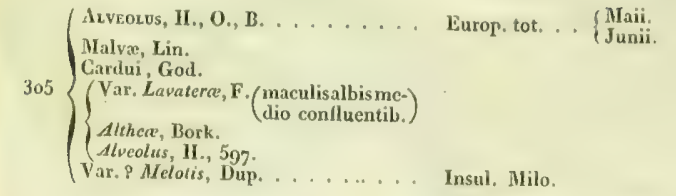

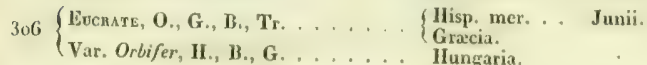

307 Tuenape, Ramb., B. . . . . . Corsica.

$308\left\{\begin{array}{l}\text { SAo, H., G., B., D. . . . . . . . } \\ \text { Sertorius, O. }\end{array}\right.$

\section{Glinus thanaOS, Boisl., Dup.}

Larva collo strangulatx. Chrysalides conicx,-Caput sat magnum, thoracis latudine. Antennarum clava fusiformis, sub-arcuata. Palpi hirsutissimi; articulo ultimo distincto, gracili. Alx integerrima, per quictem patula, horizontales, fimbria haud intersecta, fusčx, einereo undatx. Abdomen alis posticis longius.

309 Marzơ⿱ , B., D., Guenée. . . . . . Moræa.

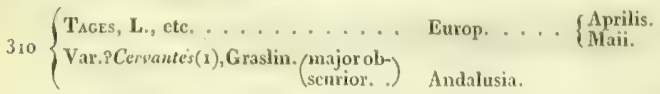

(1) Primo intuitu Than. Juvenatis et Quercus statura et colore alfinis videlur, spal si aceuratius cum $7 /$. T'agn comparetur, haud certe persuasum sst vere distinctam esse speciem. 



\section{LEGIO SECUNDA.}

\section{HETEROGERA.}

Antenna variabiles; prismaticæ, pectinatæ, serratx, noniliformes, vel filiformes. $\mathrm{Alx}$ nunquam per quictem erectix; postic sxpius frenati. Stemmata in plerisque. Volatus modo diurnus, modo crepuscularis, srpius nocturnus.

\section{LARVA PROGRESSORIE.}

\section{TRIBUS STYGIARIA.}

Larva vermiformes, decoloratx, scutellate, radices vel fruticum medullam occulte corrodentes. - Frons rotundata, squamosa. Lingui sparia, sub-nulla. Antennæ maris sub-pectinato-dentatx. Ale colorata, breviores, minime hyalina. Tibia postica valide dentata. - Volatus diurnus.

1. GENUS STYGIA, Drap., Latr., Boisd., Treits.

Chimara, Ochs.

Palpi crassi, squamati, obtusi, ultra clypeum sub-assurgentes. Antemna in utrorue sexu bi-pectinatic. Abdomen robustius, sub-elongatum, lateraliter apiceque fasciculato-cristatum.

3 II $\left\{\begin{array}{l}\text { Aosrasus, Lat, O. Iv, Tr., G., B. M Monspelii. . . Augusti. } \\ \text { Bomb. Terebellum, H. }\end{array}\right.$

Chim. Leucomelas, $\mathrm{O}, \mathrm{I1}$. 
2. GENUS CHIMERA, Oclss, Boisd. Atychice, Ilofmansegg, Latr.

Palpi squamato-pilosi, ultra clypeum assurgentes. Antennæ breves. maris sub-bipectinata, feninx ciliatx, a basi ad apicem sensim graciliores. Abdomen femina longius, oviducto exserto instructum. Femina mare minor.

312 Furguma, Fisch. ........ $\begin{aligned} & \text { Kirgisii } \\ & \text { Rossix. }\end{aligned}$

3.3 $\begin{aligned} & \text { Appendrculats, Esp., O., 1. . . . . } \\ & \text { Pyral. Vahliana, F. }\end{aligned}$

$3 \times 3\left\{\begin{array}{l}\text { Pyral, Vahliana, } \\ \text { N. Linea, Bork. }\end{array}\right.$

Sph. Chimara, II. s.

N. Chimæra, H. $314-315$.

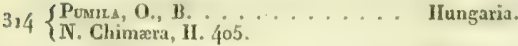

3 I5 v. Madista, 0., B. . . . . . . Austria.

316 Funeons, Feisth., B., D. . . . . Catalaunia. . Maii.

SMonspelii.

317 NAvA. Tr.

Sicilia. 


\section{TRIBUS SESIARIA, Boisd.}

Larve vermiformes, decolorat $x$, scutellate, in medulla fruticum vel radicum occulte degentes. P'uppa postice spinulosa.-Frons rotundata, squamosa, stemmatibus duobus distinetis munita. Ale plus minusve liyalinx; posticis frenatis. Aldomen cylindrico-conicum. Tibix nosticx valide calcarate, - Volatus diurnus.

1. GENUS THYRIS, Illig., Latr., Ochs., Boisd.

Palpi cylinirico-conici, ultra clypeum assurgentes, articulo ultimo sabnudo, apice sub-1nucronato. Antenna graciles, fusiformes. Ala horizontales, breves, denticulatx, fimbria intersecta, hyalino maculatix. Abdomen conicum.

318 Vituks, B., D.......... Hispania.

3i9 $\left\{\begin{array}{l}\text { Fenestrian , F., Esp., O., G., B.... } \\ \text { Pyralidiformis, H. } \\ \text { Fenestrell }\end{array}\right.$ Fenestrella, Scop.

2. GEIVUS SESIA, Lasp., Ochs., Latr:

Egeriu, Fab.

Antenna sub-lineares, vel fusiformes, apice floccido, maris interdum dentata, vel li-pectinate. Palpi squamati rel pilosi, ultra clypeum assurgentes, distincte tri-articulati. $\Lambda$ w angustissima, hyalina, vel fenestratx, stigmate ultra medium locato. Perles elongati.

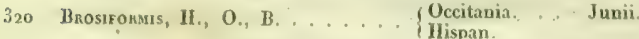

321 - Asellforms, Riossi (an var.pracedent.). Italia.

322 V.'Thetforus, O., H., B. . . . . L Lusitania.

323 Asthacirormis, lamb., B. . . . \{ \{ $\begin{aligned} & \text { Corsica. . . Maii } \\ & \text { in euphorb. }\end{aligned}$

324 Jolistirormis $(1) \ldots \ldots$ G.......... Gall. m.

(1) Allinis S. Anthrnciformis : alew anticx nigre, late fenestratac, apice fulvo sulynitentes; abdomen nigrum maculis dorsahbus fulvis, obsoletis, minine anoularibus. 
$3: 5$ \{ Phuanxumformis, Lasp., H., O., G., B. Gall. m. Musceformis, Esp.

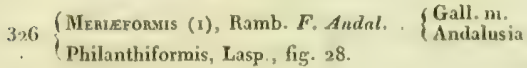

327 Mysזrsforms, Ramb. (2), N. Andal. Andalusia,

3.28 v. \{xibeuronsts, B. ....... Gall, mer.

329 $\left\{\begin{array}{l}\text { Tentukedimforms, II., Lasp., O., G. }\left\{\begin{array}{l}\text { Gallia. } \\ \text { in euphorl. }\end{array}\right. \\ \text { Empiformis, Esp. } \\ \text { Muscaformis, Bork. }\end{array}\right.$

330 y. Tengrnfarms (3), Ramb. F. Andal. Andalusia.

331 - Esptronms, H. . . . . . . Hungaria

332 - Steudifonus, Fr. . . . . . . Iungaria.

333 Tediforas, Fr. . . . . . . Hungaria.

$334^{-}$Masamiforaris, 0., B. . . . . . Austria.

335. Musczroras, H., B. . . . . . . Germania.

$336^{\circ}$ Tipeziformis, L., F., H., O. Lasp., B, G. \{ Europ. .... Junii.

337 - Sratorrohus, Fr. . . . . . . . Ilungaria.

338 - Écerferormis, O., B........ Italia.

339 - Cephroras, O., Tr., B. . . . . . Austria. ... Junii. 3.jo $\left\{\begin{array}{l}\text { Nonsozrorsus, Lasp., O., G., B. . Gallia. . . . Junii. } \\ \text { Syrphiformis, H. } \\ \text { Conopiformis, Esp. }\end{array}\right.$

(1) Valde aflinis $S$. Philanthifornis, oldistiocta, maculis fenestratis minoribus, pras-. sertim antenais in utroque sexu totis nigris. Ad hanc speciem referenta S. Philanthifornis, Cl. Laspeyres, fig. $\mathbf{2 8 ,}$ - in Gallia meridionali frequens.

(2) Fere similis $S$. Meriaformis, distincta tamen videtur, pedibus corporeque subtus pilis albis indutis.

(3) Proxima S. Tenthrediniformis ct forsan tantum varictas. Diffest a T'enlbrediniformi genuioa lined rertebrali lavo-pulverulenta. 


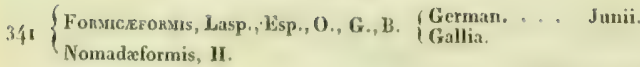

34. \{ Trmunforms, Lasp., H., O., B.... $\begin{aligned} & \text { Gallia. } \\ & \text { Italia. }\end{aligned}$

Tipuliformis, Esp.

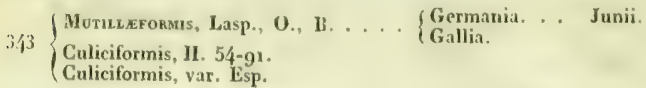

34 Cerreronms, L., O.,G.,Esp.,13,,11.151. Gall. mer. (Maii.

345 v. $\left\{\begin{array}{l}\text { Stonoxizonmis, Schr, O., H..... } \\ \text { Culiciformis, Scop. }\end{array}\right.$

346 Andeneformis, Lasp., Esp., O. . . Hungaria.

347 v.Monedulfomis, Ramb. F. Andal. Andalusia.

348 Melumfonms, Lasp., O., G. . . . Uccitania. .. Junii.

$349\left\{\begin{array}{l}\text { Crnipifonsus, H., Esp., O. . . . . . Gallis. . . . Julii. } \\ \text { Vespiformis, W., V., Lasp. }\end{array}\right.$

Var. \& OEstriformis, Esp, $\mathbf{H}$.

$350^{\circ}$ URocentronahts, Tr. . . . . . Hungaria.

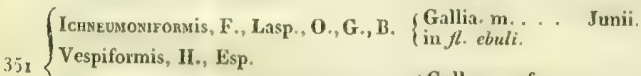

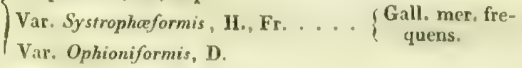

352 พ.Opmonıfonss, H. (non Dup.). ... Italia. ... Junii.

353 v. Meghlefonms. H ... . . . . Gall, mer.

$354\left\{\begin{array}{l}\text { Banchновия, H. . . . . . . Italia. } \\ \text { Empiformis, D. }\end{array}\right.$

355 v. $\left\{\begin{array}{l}\text { Pnosonironsus, } \boldsymbol{O} \text {., Tr. . . . IInngaria. . Junii. } \\ \text { Chalcidiformis, II. }\end{array}\right.$

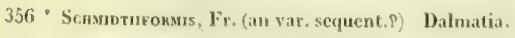




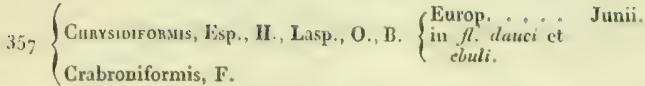

$358^{\circ}$ Donyluoras, $0 .$, Tr. ....... Lusitania.

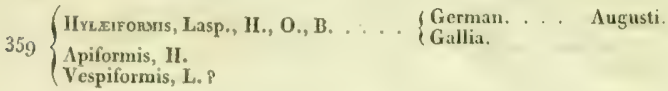

360 Scourefonas, II., Lasp., O., G., B. . \{ $\begin{aligned} & \text { Germania. } \\ & \text { Gallia. }\end{aligned}$

36r. Thynnfonus, Lasp. (an var, sequent. P), Germania.

362 Sphecronsus, II., F, Lasp., O,, G, B. Gallia. : . Junii.

$363\left\{\begin{array}{l}\text { Rungrerorms, II., O., B....... } \\ \text { Crabroniformis, Lasp. }\end{array}\right.$

364 Asulufonsns, F., Lasp., H., G., B. . . Gallia. . . . J Junii.

365 Laphrieforars, Wimm., Tr., B. . . Berolini. . . J Junii.

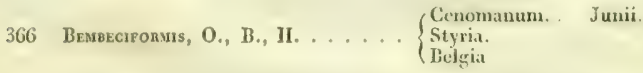

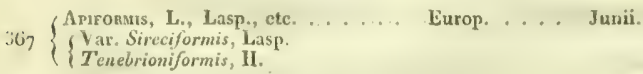




\section{TRIBUS SPIIINGIDES.}

Larvx subdiales, nudx, antice plus minusve attenuate, srepius cornu anali armatx. P'uppa cylindrico-conica, sippissime nuda, rarius sul,folliculata,-Antemes sub-lineares, prismaticx, apice tenuiore, piloso. maris subtus scohinaformes, femina simpliciores. Jalpi lati, obtusi. sapius clypeo piloso arete applicati. Alx angustx, deflexa. Alolomen maximum, conicum.

\section{GENUS MAGROGLOSSA, Ochs., Boisd.}

Sphinges, Auct. Sesia, Fab., Steph.

Larvx lxves, clongatie, capite globoso, cornu armatx. Puppa intra quisquilias sub-folliculata. - Lingua elongata, longitudinc corporis. Antennæ valde clavatæe, sub-cylindricæ. Alæ breves minus angustatæ, sxpe fenestrata. Abdomen crassum, depressum, lateribus piloso-fisciculatum, ano obtuso, barbato. Volatus diurnus, citissimus.

$+$

368 Foscifonms, L., O., 13., Tr.

Bombyliformis, F., H., G., Fr.

Gallia. ..... $\begin{aligned} & \text { Maii. } \\ & \text { Junii. } \\ & \text { Augusti }\end{aligned}$

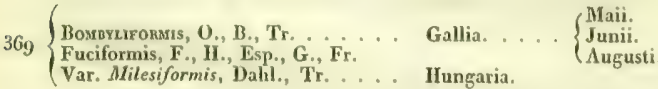

t†

$370\left\{\begin{array}{l}\text { Crostica, Esp., O., B. .......... } \\ \text { Sesia, II. }\end{array}\right.$

371 Stellatanus, $\mathbf{L}$, etc. ........ Europ. 
a GENUS PTEROGON, Boisd. Mncroglossa, Och., Tr. Sphinges, Auct.

Larva Javis, sxpe mutica, ocellata, capite mediocri, globoso. Puppa cylindrico-conica, intra folia sub-folliculata.-Lingua vix longitudine corporis. Antennic claviformes prismaticx. Alw sat breves frenatix croso-dentatx. $\Lambda$ bdomen breve, cylindricum, obtusum, apice fascicalatum. Volatus vespertiuus.

372 OEvothere, F., Esp., H., O., G. . . G Gall. mer. . . Junii.

373 Gongoniades, Boisd., II., Werzeich. . ad Volgam.

Gorgon (1), II., O., D.

3. GENUS DEILEPHIL $\Lambda$, Ochs., Boisd. Sphinges, Auct.

Larva laves, glabræ, subdiales, capite rotundato, sæpius cornu anali instructa. Puppa nuda, cylindrico-conica, humo vel quisquiliis sepulta. -Lingua distincta, capite multo longior. Antennæ valida, prismatice. Ala frenatie; anticis lanceolatis, posticis ad angulum ani tenuiter sub-productis. Abdomen elongatum, conicum. Volatus vespertinus.

广 Larva antice attenuato-rostratx, occllatx. - G. Cherocampa, D.

374 Poncersus, L., etc. . . . . . . Europ. .... Y Junii,

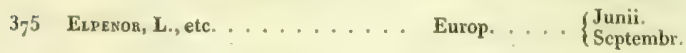

$376\left\{\begin{array}{l}\text { Alzcto, L., Cr. (3). . . . . . . } \\ \text { Cretica } \$ \text { B., D. }\end{array}\right.$

(1) Jaın nomine' Gorgon insectum ejusdem generis desigatur a Cramer, Pap. Exoliq, pl. 162, quare inutandum.

(8) In Memoires de la societe Linn. de Paris, et in Icones historique des Lipidopteres d'Europe, miscui immerito suh, nomine Cretica duas Sphinges specie diversas, judicando mares individua alis posticis cinereis, et feminas individua alis posticis rubris. Posiquam utrumque opus publicatumest, ferbinan vidi ex Gracia alis posticis cinerpis, et Gl. Bagaon Lausaniensis mares tustravit alis posticis rubris, femine instar in Memoires de la so- 
$377\left\{\begin{array}{l}\text { Cretra. . . . . . . . . . . I Ins. Grxeix. } \\ \text { Cretica of Boisd. }\end{array}\right.$

378 Osyas, Dalm., B., D. (1)...... \{ $\begin{aligned} & \text { Ilispan. mer. } \\ & \text { Algerix. }\end{aligned}$

379 Cexenro, L., etc. . . . . . . . $\begin{aligned} & \text { Hispania. ... } \\ & \text { Gall, mer. }\end{aligned}$

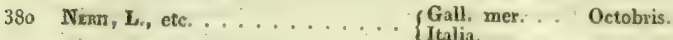

t† Laryæ haud rostratæ, lateraliter concinne maculatæ.

$381\left\{\begin{array}{l}\text { Nrcea, Deprun., O., G., B. . . . . Occitania. . . } \\ \text { Cyparissiæ, II. }\end{array}\right.$

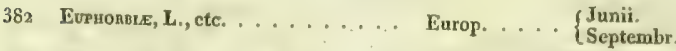

383 Esule, B; D. . . . . . . Calabria.

384 Gall, F., Esp.; II., O., G. . . . . \{ \{ $\begin{aligned} & \text { Gallia..... Junii. } \\ & \text { German. }\end{aligned}$

384 LivoNica

385 DauLu, Tr., B., D., II. \{Corsica....

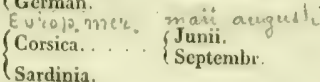

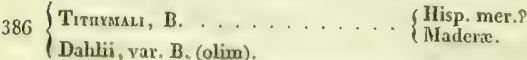

387 Zygopnyuli, H., О, B., D. . . . . . Russ. mer.

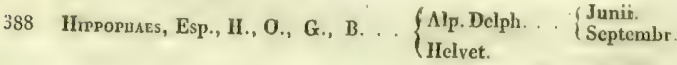

389 EPLLOBI, B. . . . . . . . Lugduni.

$3_{90}\left\{\begin{array}{l}\text { Vespentuloides, B. . . . . A . Delph. } \\ \text { Amclia, Feisth. D. }\end{array}\right.$

cielt Linn, figurata. Itaque nomen Cretica relinquo speciei quam marem esse credideram, quaque nova est. Individua autem alis posticis rubris io utroque sexu, jam secerni non possunt a $S_{p h}$.Alecto Crameri qua degit in Bengalia, queque, ut $S p h$. Nerï, in finibus Europa decrescit el desinit.

(t) Hane speciezn in Hispania occurrisse plane compertun non habeo. D. Goudot, a quo illam accepi, cum aliquandiu Tanger commoratus fuerit, potest illam in his locis legisse et miscuisse cum insectis qua per Hispaniam iter faciens legit. 


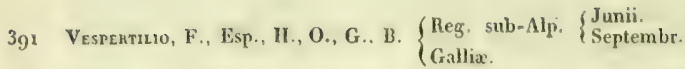

Nota. Quamy is din in dubium vocata fuerit generatio hybricla in libertate, hodie manifestis constat argumentis, D. Vespertilio copulare natura cum quibusdam aflinibus specicbus qux cosdem loros inhalitant. Ita D. Epilobii nascitur ex atulterino congressu D. Euphorbice cum I espertilio, et I espertilioüles ex copulatione Ifippophaes cum eadem illa specie. Larva non multa numero, qux ex is congressibus oriuntur, moto in planta propria D. Vespertilio (Epilobium Rosmarinifo$(\mathrm{ium})$ pascuntur, modo in ambabus alis spcciclus propria, prout mas ant femina ad istam copulationem notham se admovit. Caterum hybride illa rarissime occurrunt, nec sese tegenerant, nec ullum signum speciale in illis eminct, sed proprii utrique specici characteres fusi omnino et mixti cernuntur. $\Lambda$ pud $Z$ ygarnas quoque, sxpius quidem, hybridas videre est; unde non semel dificillime cas notis certis definire.

Atque hic intempestive non dixcrim, Cl. Duponchel erravisse, cum professus est nomen Amelia, uni ex illis hybridis inclitum, anterius esse nomine Vespertilioïdes, qui, si curavisset adire Mémoires de la Soc. Linn. de Paris, et cum titulo temporis, Bulletin de Férussac comparare, non in hunc crrorem incidisset.

\section{GENUS SPHINX, Ochs., Boisd.}

Sphinges, $\Lambda$ uct.

Larvx laves, subdiales, oblique fasciat $x$, capitc rotundato, cornu anali instructe. Puppa nuda cylindrico-conica, apice mucronata, vagina pectorali ansa-formi predita, lumo sepulta.-Lingua corpore longior, antennx validx, scobinxformes. Alx frenate; anticis lanccolatis, posticis ad angulum analem rotundatis. Abdomen cylindrico-conicum, cingulatum. Volatus vespertinus.

392 Pinssta, L., etc. ......... Gall.or.etmer. Junii.

393 (Ligosta, L., etc. . . . . . . . Europ. . . . Junii.

(Var. Spirea, H.

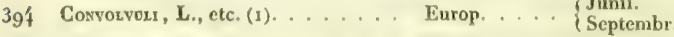

(1) Cl. Dom. Stephens (systematic Catalogue of British insects) enumerat ut insecta Anglica, scilicet : Sphinse Carolina, Line.;-Quinquemaculatus, Haworth, Curtis; Connolvuli, var. Smith-Abbot; - Pleteja, Fabs; - Peciln, Steph.; -Daucus, Cram.;Afgentata, Steph. Illx omoes Sppinges Americam borealem vel Africam inhabitant, ot immerito Anglie adseriptie sunt. 


\section{GENUS ACHERONTIA, Och, Brachyglossa, Boisd., Otim.}

Lat we leves, oblique fasciatx, capite rotundato, cornu anali granuloso, caudicformi instructa. P'uppa cylindrico-conica, sub-obtusa, apice bi-mucronata. - Lingua crassa, pectore brevior. Palpi breves, ohtusissimi. Caput crassum. Antenna breviores, rigidx. Alex frenatx; anticis lanceolatis, posticis ad angulum ani rotundatis. Abdomen crassissimum, minus conicum. Pedes valide unguiculati. - Volates gravis, vespertiuus.

Nota. Brachyglosse geuuina Australasix, sat discrepant a Sph. Airopos et affinibus ut al illis secernantur; quare genus Acherontin Ochscnilreimeri servandum et immerito a celcliers. Latreille specicbus Nova-Hollandix translatum, censemus.

$395 \quad$ Atropos, Lin., ctc. ....... Europ. ...

\section{GENUS SMERINTHUS, Ochs., Latr., Boisel. \\ Dilinn, Dalm. Sphinges, Auct.; Vet.}

Larva scabrosx, capite triangulari, cornu anali instructa. P'uppe nudx, cylindrico-conic $x$, terra sepultx. - Caput parrum, retractum. Palpi brevissimi, obtusi, ultra oculos haud assurgentes. Jingua bres issima, spuria, vix ulla. Antenna sub-lineares, sub-flexuose, naris subtus dentat ; femine simplices. Ala haud frenate, margine angulosodentatx. - Volatus gravis, nocturnus.

Nota. Hocce genus Sphingidum ad Bombyces Auct. transituı evidenter efformat.

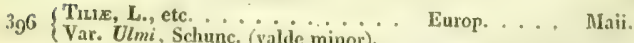

$3_{97}\left\{\begin{array}{l}\text { Oceltats, L., etc. . . . . . . . E Europ. . . . } \\ \text { Salicis, H. }\end{array}\right.$

398 Poptr, L., etc. ......... Europ. ... \{ Maii.

399 ч.TRenul. Zetter, Tr., H., D. . . Moscou.

joo. Qoencos, F., Esp., H., O., B. .... $\left\{\begin{array}{l}\text { Iungar. ... Maii. } \\ \text { Austria. } \\ \text { Gall, mer. }\end{array}\right.$ 


\section{XIY. TRIBUS ZYG ENIDES.}

Larva sub-contracte, pigra, capite minuto, pubescentes vel pilosula. Puppa folliculatx. - Palpi cylindracei, villosuli ; articulo ultimo distincto, sxpe nudo. Fronssquamosa. Antenna basi graciliores, flexuosoclavatic, rarius filiformes vel bi-pectinatte. Al.e antice angustiore's postice rotundata. Abdomen cylindricum, sub-lineare. - Volatus diurnus.

1. GENUS ZYGNENA, Latr., Ochs., Boisd.

$$
\text { Anthrocera, Scop., Stepl.. }
$$

Larwx contracte, sub-cylindrica pubcscentes. Puppa folliculo chartaceo, fusiformi, vel crastaceo ovoideo, inclusa.-Intenne flexuoso-clitvata, dimidio corporis longiores, apice imberbi. Palpi breves, cylindricoronici, pilosi. $\Lambda$ l $x$ elongat $x$, maculata. Pedes mediocres, obsulcte calcarati.

\section{$+$}

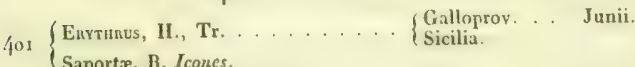

(Saportxe, B. Icones.

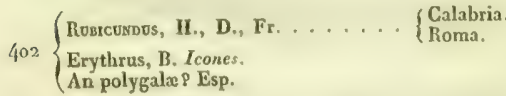

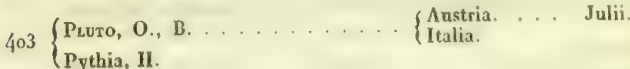

(Muxos, W. V., H., O., B. ...... G Gallia. .... Julii

$404\{$ Pilosellix, Esp.

Scabiosin.

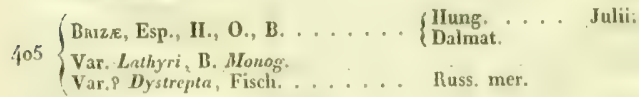




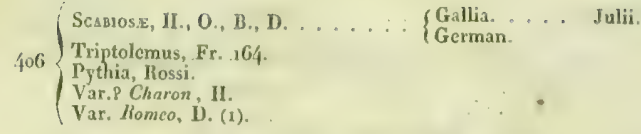

407 Dambitina, B. Icones, D. (2). . . Dalmatia.

Var, Nevadenis, Ramb. . . . . Hispan. mer.

408 Puncruar, O., B., H. : . . . . . . E Europ. mex, Julii.

409 Contamuket, B., D. . . . . . . . Pyrén. .... Julii.

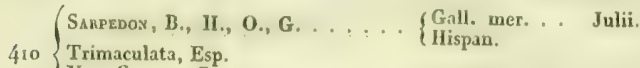

Var. Cynare, G.

Var. Balenrica, B. (3). . . . . . . Andalusia.

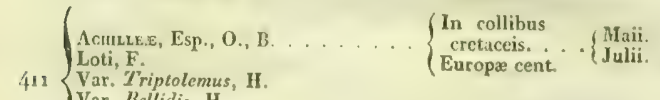

Var. Bellidis, $\mathbf{H}$.

Var. Vicia, H.

Vas. Cytisi, Fr. 164.

412 Jantuna, B., D........... Monspel. . Julii.

\section{t十}

413 Bitoreuats, Ménétr. (an var. seqquent,8) Caucasus. . Julii.

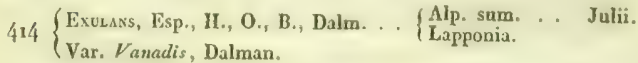

$415\left\{\begin{array}{l}\text { Crnare, Esp., H., O., B. (non God.). } \begin{array}{l}\text { Italia. .... Julii. } \\ \text { Ilungaria. }\end{array} \\ \text { Millefolii, Esp. }\end{array}\right.$

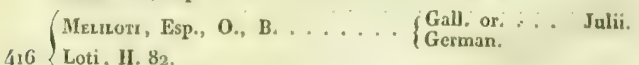

$416\left\{\begin{array}{l}\text { Loti, H. } 82 . \\ \text { Vicix, Bork., II. }\end{array}\right.$

Var. Buglossi, Escher., D.

(1) Z. Snmeo Cl. Dupoochelii ad Z. Seabiosa certe referenda : bae varictas uaculis interruptis occurrit frequens, et ab auctoribus plerisque descripta.

(थ) Discrepat a Z, Sabiosa prasertion anteonis multo crassioribus, fere ut in Z. Punetum. An hybrida?

(3) Hæc vorietas ud Zrg. Punctum prope accedit et vix nisi annulo rubro distiocta inde ad Sarpedon referenda. An hybrida P 
487 Danurca, B., D. ......... Sibiria.

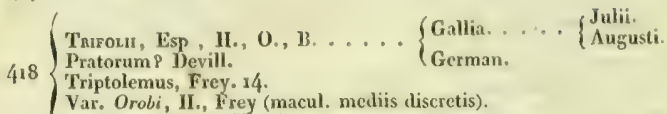

Var. Orobi, II., Frey (macul, mediis discretis).

Var. Glycyrrhiza, II., Fr.

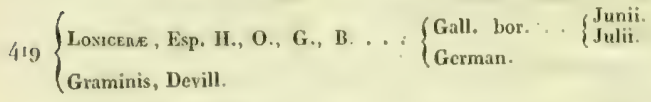

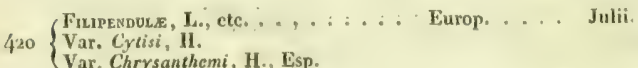

(Var. Chrysanthemi, H., Esp.

$\left\{21\left\{\begin{array}{l}\text { Transazpina, H., O, B., D. }\left(\begin{array}{l}\text { an var. } \\ \text { maj.prx- } \\ \text { cedent.P }\end{array}\right) \text { \{ Gall. mer. } \\ \text { Filipendulia. }\end{array}\right.\right.$

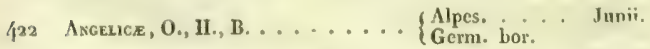

$423\left\{\begin{array}{l}\text { IIIppockepidis, O., B., G., Frey. . Gall. cent. . . Julii. } \\ \text { Loti, Esp, II. 32. } \\ \text { Ab. Ilippocrepidis, (II. } 83 \text { macul, alb. } \Lambda \text { Sis posticis flav.). }\end{array}\right.$

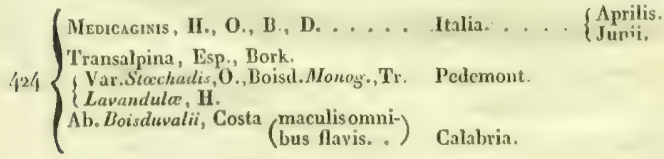

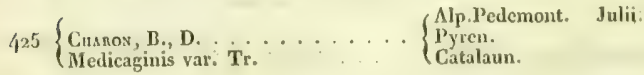

$426^{\circ}$ Centauree, Fisch.......... Russ. m.

427 Dorycisir, O., Tr., B., D. . . . . . Russ. m. . . Junii.

(Peucedant, Esp., II., O., G., B. . . Europ. cent. . Julii.

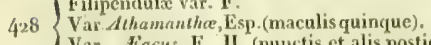

Var. Eacus, F., II. (punctis et alis posticis flavis).

Varr. Eacus, Esp. (posticis flav. punct. 3 disci albis). 
ZYG ENIDES .

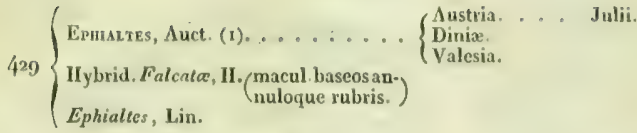

$430\left\{\begin{array}{l}\text { Isyandele, F., O., G., B. ...... } \\ \text { Spice, H. }\end{array}\right.$

ttt

431 Stoechadr, B., D. (non alior. auctor.). Catalaun.

432 Rhrabamanthus, Esp., 1I., O., G., D. $\left\{\begin{array}{l}\text { Gall. m. . . J Julii. } \\ \text { Hispania. }\end{array}\right.$

433 Oxyrkopis, B., Tr, D., Fr. . . . Etruria..... Junii.

434 Ouıven, 13., Ménétriés. ....... Caucasus.

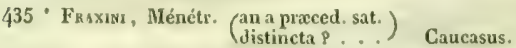

436 - Scovitzı, Ménétriés. . . . . . R Russ. mer.

437 Sed, F., II., O., Tr. ....... Russia mer

Onobrychis, var. B. Monog.

Onounxcus, F, H., O, G., B.... Europ. cent. . Julii. Caflira, Esp.

438 Var. Ilcdysari, $1 \mathrm{H}$.

Var, Astragali, II.

Var. Meliloti, II.

Ab. Flaveola, II., Esp.

$4^{3} 9\left\{\begin{array}{l}\text { Occitantca, Devill, O., G., B. . . . } \begin{array}{l}\text { Occitania. } \\ \text { Hispania. }\end{array} \\ \text { Phacx, H. Julii. }\end{array}\right.$

440 v. FaUstina, O., B., Tr. ........ Hispania.

441 Bетіс, R. (an a præced. sat, dist. P). Ilispan. mer.

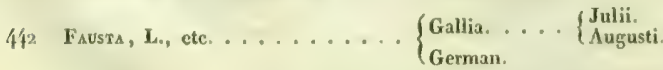

(1) Celeb. Dom.Treitschke lepidopterologus eximius, in hanc opecien cum Z. Filipendula copulantem identidem jocidit; isto congressu adulterioo oriuntur varietates notba Faleata, H, Ephialtes, L. Quac cingulo et maculis basilaribus rubris gaudent. 


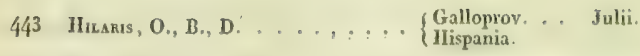

494 LETA, Esp., H., O., B. . . . . . I Iungaria.

445 Asthrumids, B., D. . . . . . P. Pyren. . . . Julii.

446 Corsics, B., Ramb., D ... \{ $\begin{aligned} & \text { Corsica. . . Junii. } \\ & \text { Sartinia. }\end{aligned}$

\section{GENUS SYNTOMIS. Illig., Latr., Ochs,, Boisd.}

Larva cylindrica vel cylindrico-ovata, pilosax. Puppax folliculo molli involutx. - Antennx lineares, graciles, sub-fusiformes, dinidio corporis longiores. 1'alpi breves, sub-cylindrici, obtusi, breves. Ala clongatx, maculatx. Pedes mediocres, obsolete calearati.

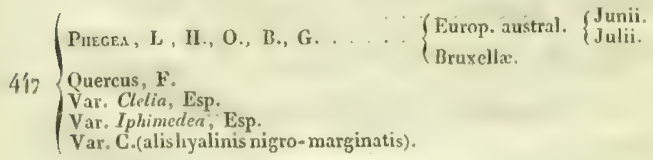

3. GENUS PROCRIS, Fab., Latr., Boisd., God.

Atychia, Ochs.

Larve ovatx, contractx, temuissine villosa, sub-depressa. Puppox folliculatx.-Antenna sub-lineares, apice aut incrassato, aut cuspidato, subtus in mare distincte bipectinatx, vel totre vel ex parte, in femina obsoletius dentata vel sub-lxves. Palpi graciles, clypeo breviores. Alac haul maculatx.

$448\left\{\begin{array}{l}\text { Siatices, L., F., ctc. . . . . E Europ. . . . Julii. } \\ \text { Var. Hhican, Frcy. } \\ \text { Var. Geryon, II. }\end{array}\right.$

419 Cognata, Ramb. Faun. And........ Hispan. mer.

450 Grobchare, Esp., II., O. . . . . Gallia. . . J Julii.

Var. Chloros, 11.

$451\left\{\begin{array}{l}\text { Amrelopuags, 11. Tr., 1)..... } \\ \text { Vitis, 13., Frey. }\end{array}\right.$ 
452 PaUnt, F., W. V., O., G., B.... Gallia..... Junii.

453 S.epius, B. Icones, D. . . . . . Lombard. $454\left\{\begin{array}{l}\text { Infadsta, L., etc. . . . . . . . Gall. m. . . Janii. } \\ \text { (Gen, Aglaope, Latr.) }\end{array}\right.$

\section{GENUS HETEROGYNIS, Rambur.}

Tinea, Hubn.

Larva tenue pubescentes, contractx, onisciformes, subdiales. Puppa folliculo retiformi inclusa. Metamorphoses feminx sub-nullax; illius imago a larva nisi pelle nova discrepans, in folliculo copulans ovaque parens. - Mas habitu ad Procridesaccedens : antennæ sul-lineares, pectinate, apice cuspidatx. Lingua brevior. Palpi brevissimi, villosi. Corpus gracilius. $\Lambda$ le latiores, diaphano-fuliginosæ ut apud $P_{\text {sychiles. }}$ Volatus diurnus. - Hocce genus primo intuitu a Psychidibus differt antennis longioribus alisque magis elongatis.

Dom. Rambur species quatuor hujus generis descripsit, sed oculo acutissimo vix tres visendx.

455 Penelra, Hubn., fig. 447, Ramb. Alp. Diniæ. Augusti.

456 Parudoxa, Ramb.......... Sierra nevada. $\begin{aligned} & \text { Augusti. } \\ & \text { Septembr }\end{aligned}$

45. Arrins, Ramb. (an sat. distinct.8). .. Andalusiz.

457 Var. Hispana, Ramb. ....... Catalaun. 


\section{TRIBUS LITIIOSIDES, Boisd.}

\section{Chelonarii, Boisd., Olim.}

Latwe herhivere, vel lichenirorx, elorso pilis fasciculatis vestite. Puppa breviuscular, sxpius sub-contracter, segmentis immobilibus. - Alir inticar per cquictem dorso decussita, postice plicate, abdomen subinvolycntes. Corpus gracile, elongatum.

\section{x, GENUS EUCHELIA, Boisd. \\ Callimorpha, Latr., Steph. Dejopeia, Curt, Steph.}

Latra herhivora, clongatx, pilis rariusculis vestit $x$, sub-gregaria, anunlato-fasciatic, vel transversim maculatx. - Antenna in utrorge sexu simplices. Palpi hreves, tenne pilosi, distantes; articulo altimo ohtuso, distincto. Lingua convoluta, sat producta. Ale latiores, concienc rubro vel cocciuco maculatix. - Volates diurnus.

$$
+ \text { Ala strigata. }
$$

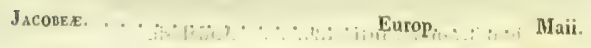

if Alx punctatx:

$450\left\{\begin{array}{l}\text { Puccura, Esp., H., O., B. . . . . G Gall. m. . . Junii. } \\ \text { Pulchella, L., G. } \\ \text { Lotrix, Cram. }\end{array}\right.$

a GeNuS EMYOLA, Boist.

Eulepia, Steph.

Latrva graminivorx, subdiales, obscur $x$, vittis dilutioribus ant coloratis. l'uppa contracta, femiux ventricosa. - Antennx sat clongata, maris pectinat.e, femine ciliatx. Palpi capitebreviores, Limgua , 'istincta. Ala l.tiores, antice nigro punctatxe vel strigosx. Corpus gracile. Abelonen clongatum. - Volatus diurnus. 
$461\left\{\begin{array}{l}\text { Coscunıs }, 0, \text { B. . . . . . . } \\ \text { Chrysocephala , 1I. }\end{array}\right.$

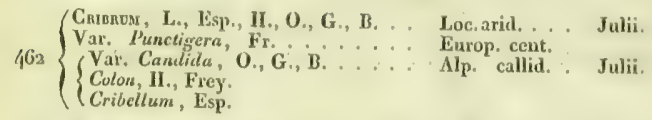

463 Bifásciata, Itamb;, B. ( $\left.\begin{array}{l}\text { An sat a pre- } \\ \text { ced. distincta? }\end{array}\right)$ Corsica. ... Junii.

46 Jitpertii, B., D.......... Pyren. ... Julii.

$465\left\{\begin{array}{l}\text { Gnamarca, L., II., O., G. . . . . . Europ. . . . Julii. } \\ \text { Var. A. (alis posticis nigris). }\end{array}\right.$

\section{GENUS MELASINA, Boisu.}

Eyprepia, Ochs.

Larva ignota. - Antenna sat clongata, maris pectinate, fenimx ciliatx. Palpi her issimi, valde pilosi. Lingua spuria, vix ulla. Statura robustior. Ala ongustae, immaculat $x$. Corpus robustius, alis posticis longius.

$\left\{66\left\{\begin{array}{l}\text { Cıtıиs, O., D. Ind. meth. (1). . . } \begin{array}{l}\text { Valesia. } \\ \text { Dalmat. }\end{array} \text { Lugubris, 1I. 216. }\end{array}\right.\right.$

\section{GENUS LITHOSIA, Boist.}

Lithosie et Callimorphe, Latr. Setina, Schranck.

Larralichenivora, obscura, subdtales, unicolores, vel lutcoalhoque maculate. - Caput parvum, squamatum. Palpi breves . Lingua modice dongata. Autenna filiformes. Ala elongate, angustiores. Abdomen clongatum, sæpe alis posticis longius. Feminæ statura mares adxquantes aut superantes.

(1) Tota atra, ciliis alarum margineque costali anticarum albis.

Tres in collectionibus confusw sunt species nomine Lugubris; scilicet : Eyp. Ciliaris, Ochs., Chis. Lugubris, Ochs et Typhonia Melas, Boisd, omnes plane distincta quanvis primo'intuitu fere similes. P'rina (M:Ciliaris) antennis maris' peclinatis abonde distincta a speciebus generis Typhonir; secubda (Typh. Lugubris) manileste discrepat a $T_{y p h}$. Melas fubbria alarum albida. Vid, Gen. Typhenia, pag. 78. 
$+$

467 Reвrrcorrs, L., etc......... Gallia. .... Maii.

tt

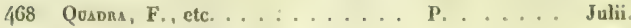

tt

469 Gruseors, H., O., B. . . . . . . Gallia bor. . Julii.

470 \{ontpsasa, L., F, , ., G. . . . . Europa cent. . Julii.

47 (Complasuls, Boisd, D. (frequentior)(1). P. . ..... Junii.

Lurideola, Tr.

472 Cantor, II , O., B. ........ $\begin{aligned} & \text { Italia...... Junii. } \\ & \text { Gall. mer. } \\ & \text { I. }\end{aligned}$

$47^{3}$ Albeols, 11. ( an var. proced, ?). . . Italia.

474 Lacteola, B., D. . . . . . . . . Corsica.

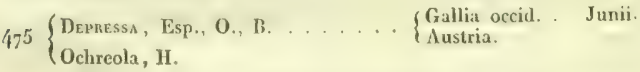

$4-6\{$ Hetreora, O., B. . . . . . . Gallia. .... Junii.

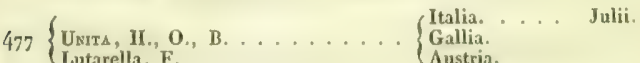

Lutarella, F.

4,8 Gllveola, O., B. . . . . . . . Austria.

(Loteola, H., O., B. . . . . . . Gallia . ... Junii.

$179\left\{\begin{array}{l}\text { Lutarella, L. } \\ \text { Lutarella, var. }\end{array}\right.$

$480 \quad$ Vitellina, Tr., B. . . . . . . $\begin{aligned} & \text { Gallia..... Augusti } \\ & \text { Austria. }\end{aligned}$

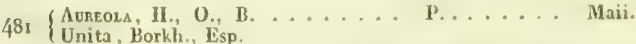

(1) Plerique auctores ambas species sub una commislas (Complona) ajoe dubio pro oculis habuerust. 


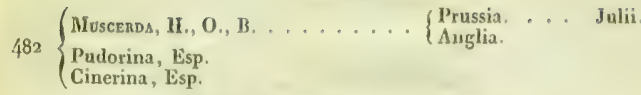

$483\left\{\begin{array}{l}\text { Mesogona, G. Pap, de France. . . . Corsica. } \\ \text { Var, Rufeoln, R., B. Icones. }\end{array}\right.$

t+t Larva dense lanatx.

$484\left\{\begin{array}{l}\text { Rossa, } \mathbf{F}_{1}, \mathbf{O}_{\text {, }} \text { G., B. . . . . . . Europ. . . . Junii. } \\ \text { Rubicunda, If. }\end{array}\right.$

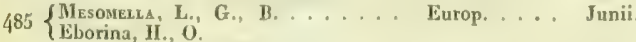

5. GENUS SETINA, Boisd, Steph.

Setine, Schrank. Lithosia, Ochs. Callimorphoe, Latr.

Larvæ subdiales, obscuræ, pilis fasciculatis vestitæ. Puppa contracta, sub-ventricosa. - Antenna maris ciliata, feminæ simplices. Palpi breves, squamati. $\Lambda$ læ deflexæ, minime decussatæ, in feminis manifeste lreviores, suh-imperfecto, volatu vix proprix; in utroque sexu flavx, nigro punctatæ aut strigosæ.

486 Roscuma, F., H., O., G., B. . . . . Helvet. . . . Julii.

$487\left\{\begin{array}{l}\text { Innones, H. }, \text { O., G., B. . . . . } \\ \text { Inorella, L. }\end{array}\right.$

488 Funicans, B., (Dinix. .... Junii

489 Kuilwermu, B., Tr., II

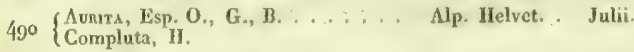

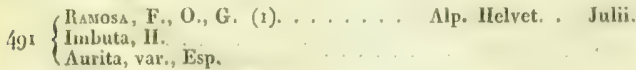

$49^{*}$ Avrara, Ménétriés. . . . . . . Caucasus.

(b) Aurita el Ranosa simul copulantes non semel obvia, unde varielates hybridar, ister Auritam of Ramosam mediac, oriuntur, - Dom. Anderregg mecum counmunicavit varietatem hybridam una hermaphroditum quax o destra Ramose mas ol a sinistra furila femina. 
6. GENUS NACLIA, Boisd. (1). Callimorphe, Latr. Lithosia, Ochs.

Larva subdiales, lichenivorx, obseure flavo maculate. Puppa contracti. - Antenna longiores, setiformes, maris vix suls-ciliata. Alx decussata, antic $x$ lanceolatx, sub-latiores, maculis semi-diaphanis signatx; postice brevissima. Abdomen punctatum. Feminx mares adxquantes.

$49^{3}\left\{\begin{array}{l}\text { Anculs, I., II., O., G., B. . . . . } \\ \text { Obscura, F. } \\ \text { Var. A. (alis anticis impunctatis). . . . Julii. }\end{array}\right.$

494 Fasurs, Fr.(an sat a præced distinct.P) Datmatia.

$49^{5}$ (Punctata, F., O., B. . . . . . . Gall. m. . J Junii.

$49^{5}$ Serva, II., G.

7. GENUS NUDARIA, Stephens, Boisd.

Callimorphe, Latr. Lithosia, Ochs.

Ian va subdiales, lichenivora, olscura, - Antenna setiformes, longiores, maris vix ciliat $x$. $\Lambda$ lxe omnes deflex $x$, livid $x$, latiores, rotundatx, semi-ditphana, strigis tranversis obscurioribus. - Feninie masculos adxụuantes.

496 Sexex, H., O., B., G. . . . .... Găllia . ... Julii.

$497^{\circ}$ P Pinqganed, II. (an hujus generis?). Hispan.

/198 • Semans, II. (an hujus generis?). . Hispan.

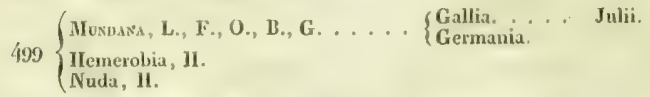

500 Mlums, Esp., II., O., G., B. .... Gallia. . . . J Julii.

(1) Hoce genus quamiam aflinitatcm cum Syntomidibus probet. 


\section{TRIBUS CHELONIDES, Boist.}

Chclonarii, Boisd, Olim.

Larva sapius solitares, pilis fasciculatis ilense vestitix, sxpius plantas humiles pascerites, polyphagx, lubricipedes, Puppa folliculata:"Antennæ mavis, feminæ obsoletius, pectinatæ, aut ciliatx. Alæ deflexæ, concinne depictre. Insectum robustum. Abdomen crassius, maculatum. - Volatus sæpius gravis, nocturaus.

$x$, GENUS CALLIMORPHA, Boisd.

Callimorphe, Latr. Eyprepia, Ochs. II Ypercompa, Steph.

Larvæ per diem sapius latentes. - Caput parvum, squamosum. Antenna clongatx, in utroruc sexu setiformes, tenue ciliatx. Palpi rapite sub-breviores, sub-listantes. Lingut distincti, convoluta. Thorax mediocris, squamosus. Abdomen elongatum, cylindricum, merliocre.

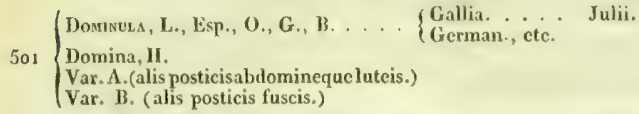

(Donka, Esp., B., D. (abdom, cyaneo). Italia. . ... Junii.

502 P'ersona, H. Beytr., Tr., H. Icon. 319.

$\left\{\begin{array}{l}\text { Domina, II, } 223 . \\ \text { Var. A. (alis posticis rubro-radiatis; abdom, cyanco). }\end{array}\right.$

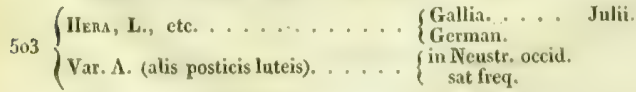


2. GENUS TRICHOSOMA, Ramb., Boisd.

Eyprepin, Tr.

Larva sub-gregarix. Puppa breves, segmentis immolilibus. - Antennx maris pectinatx, femin $x$ ciliatæ. Palpi hirsuti. Lingua distincta. Statura robustior. Ale femine spurix, - Volatus maris meridianus.

504 Consicux, Ramb., B., D., Tr. . . Corsica.

505 Beтicur, Ramb., B., D. . . . . . Hispan, mer.

506 Pakasitom, Esp., H., O., G., B.... \{ $\begin{aligned} & \text { Ilungar. } \\ & \text { Valesia. }\end{aligned}$

3. GENUS NEMEOPHILA, Stephens. Chelonia, Boisd., Olim. Eyprepia, Oclus.

Larvx per diem latentes. - P.ulpi pilosi, hreves. Lingua gracilis, distincta . Antennx maris pectinata, fenina ciliate. Statura ilebilior. Femina masculo sub-crassior, minor. - Volatus maris diurnus.

+ Alx unicolores stigmate discoidali. G. Euthemonia, Steph.

$507\left\{\begin{array}{l}\text { RussulA, L., F., H., O., G, B. ... Europ. ..... } \\ \text { ơ Sannio, L. }\end{array}\right.$

tt Alx rivulatx. G. Nemeophila, Steph.

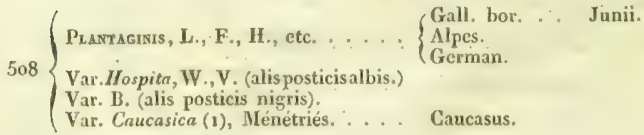

(1) Chelonia Caucrsica Mpinetriés ab individuis vulgaribus differt, maculis flayi discretis, latioribus, collari toto costaque rubris. 
4. GENUS CIELONI A, Latr., God, Boisd. Eyprepia, Ochs. Arctia, Steph. Arctia, Latr., Olim.

Larve solitares.-Palpi piloso-sruamosi, mediocres, in rostrulum inflexum producti. Lingua spuria, vix ulla. Antennx maris pectinat $x$ vel dentatx, feminx sub-dentatx. Statura crassior. Alx anticx, rivulosa vel maculatx; postic lutex rel rubre, maculatx. Feminx mares adrequantes vel superantes. - Volatus nocturnus.

$509 v \cdot\left\{\begin{array}{l}\text { Quessel, Paykull., Schneid, D. . } \\ \text { B. Strigosa, F. }\end{array}\right.$

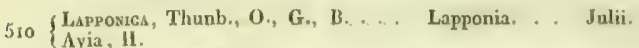

5 i Aoura, L., F., O., H, G., B. . . . \{ Austria. . . Junii.

$512\{$ Crvic, H., G., B., D. . . . . . Gallia..... Junii.

$5 i^{2}$ Curialis, 0.

513 Dejeaku, G., B. . . . . . . H Hispan.

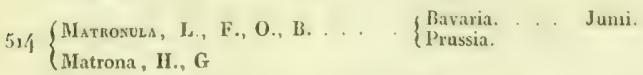

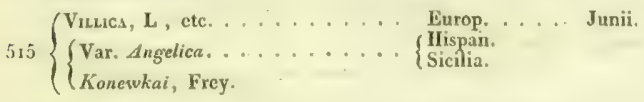

516 y. $\left\{\begin{array}{l}\text { Tuulra, Dalm, (s). . . . . . Lapponia. } \\ \text { Mlpina? Acerbi. }\end{array}\right.$

$517\{$ Fisciats, Esp., O., G., B. . . . . Gall. mer... Julii.

5 IS Latreirli, G., B., D. . . . . . C. Catalaunia.

519 Pudrca, Esp., II., O., G., B. ..... $\begin{aligned} & \text { Gall. mer ... } \begin{array}{l}\text { Maii. } \\ \text { Junii. }\end{array} \\ & \text { Italia. }\end{aligned}$

(1) AFis onticis nigris, fisciis uacularibus altis ; posticis rubris, basi fasciaque intramarmiali inequali nigris; corpore nigro, collari anoque flavidis; pedibus rubro annulatis. - Habilat ad Enontchis Lapponix. 
520 Danumca (1), B. Iconcs. . . . . . . Sibiria.

$525\{$ Ponpunes, L. ............. Gallia. ... Junii. Var. $\boldsymbol{\Lambda}$. (alis posticis luteis).

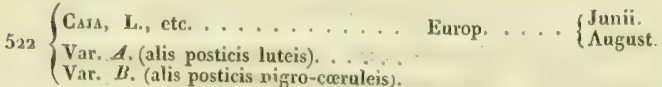

$523\left\{\begin{array}{l}\text { Fravis (2), Esp., F., O., G., B. . . } \begin{array}{l}\text { Sibiria, } \\ \text { Helvetia. }\end{array} \text { Virgo, H. . Junii. } \\ \text {. }\end{array}\right.$

$52\}\left\{\begin{array}{l}\text { IIrac, L., etc. . . . . . . . . . } \\ \text { Var. } \Lambda \text {. (alis posticis flavis). }\end{array}\right.$

525 Casta, F., H., O., G., B. ...... IIungat. ... Junii.

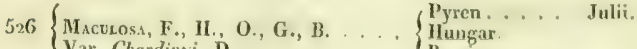
Var. Chardinyi, D.

527 Stmplonica, Anderregg (3), . . . . Valesia..... Junii.

528 v.Zonama, Graslin., D. . . . . . : : Mispan. mer.

\section{GENUS ARCTIA, Boisd.}

Chelonice, Latr, God. Arctice, Latr, Olim. Eyprepin, Ochs. Phragmatobia et Spilosoma, Steph.

Larve solitares, lubricipedes. - Palpi breves, distantes, distinctissimi, valde inflexi, pilosi, vel sub-squamati, nudiusculi. Antema maris pertinate vel ciliatx, femina sub-filiformes. Statura mediocris. $\Lambda$ la intica unicolores, immaculatx, vel nigro punctat $x$. lemine mares adæquantes. - Volatus nocturnus.

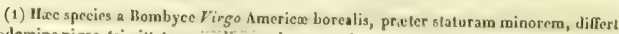
abdomine nigro tri-vittato, maculisque alarum antiearum minoribus.

(3) Specimina ex Helvetia majora, fere Matronula æqualia.

(3) Aftinis certe Maculese, at plane distincta ontennis multo angustioribus. Alac antica ut apud $T^{\prime}$. Parasitum; posticar rosex, fascia basali obsoleta, maenla centrali uargioeque Jatiori nigris. 
+ Abdomen punctis nigris trifariis. G. Phragmatobia, Steph.

5 ig Fouginosa, L., etc. . . . . . . . E Europ. .... Junii.

Septembr.

530 Luctifera, F., II., O., G., B. ... $\begin{aligned} & \text { Gallia mer. . Julii. } \\ & \text { Helvet. } \\ & \text { Austria. }\end{aligned}$

t† $\Lambda$ bdomen punctis nigris quinquefariis. G. Spilosoma, Steph.

531 Rivoxaris (1), Ménétriés. . . . . . Russ. nor. . Sentembr.

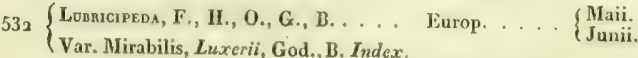

$533\left\{\begin{array}{l}\text { Unrices, Esp., II., O., G., B. . . . . Europ. bor. . J Junii. } \\ \text { Papyratia, Curt., Marsh. }\end{array}\right.$

534 \{ Menthastri, F., H., O., G., B. . . Europ. ..... Junii.

Var. Walkerii, Curtis (punctis nigris radiatim confluent.).

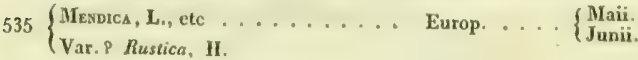

536 Lucruosa, H., Tr. ......... $\left\{\begin{array}{l}\text { Sicilia. } \\ \text { Turcia. }\end{array}\right.$

$+\dagger+\Lambda$ bdomen impunctatum.

53 . Sosdids, H., B......... $\begin{aligned} & \text { Italia bor. } \\ & \text { Ilelvetia mer. } \\ & \text { Alp. Provinc. }\end{aligned}$

(1) Alis angustioribus, albis; anticis maculis 14, triangulari-inaqualibus, atris; posticis bi-maculatis; thorace flavescente; abdomine lutescente punctis nigris quibque-seriatis. 


\section{TRIBUS LIPARIDES, Boisd.}

Larve pigra, sxpius arboricolæe, pilis fasciculato-tuberculatis vestitx, tuberculo primo laterali, alteris magis producto, longius pilose; lorso postico vesiculis duabus retractilibus pradito. I'uppa laxe folliculate, pilis fasciculatis sxpius adsperse. - Ma semi-deflexa. Antennæ maris valde, feminæ obsoletius pectinatx vel dentate. Abdomen maris mediocre, feminæ obesum.-Volatus maris sxpius diurnus.

1. GEIVUS LIPARIS, Ochs., Boisd.

Bombyces, Auct. Hypogymna, Psilurn; Penthophern, Porthesia et Leucoma, Steph.

Larve sub-depressa, tuberculis stellatim rigideque pilosis; pilis lateralibus densioribus. Puppe laxe sub-folliculatx, sappius sub-nuda. $-\mathbf{\Lambda n -}$ tenne maris valde, feminæ obsoletius pectinatie. Lingua nulla. Ala unicolores, vel strigis transversis punctisque aliquot fuscis signatx. Abdomen feminx crassum, lana anali sapius obtectum.

+ Alæ nigræ sub-desquamatx. G. Pcnthophern, Steph.

538 Mono, L., Ochs., ctc. . . . . . . Austria.

Var. Pentophera Nigricans, Curt.

t† Alæ strigis transversis, ohscuris signatæ. G. Penthophern, Mypogymu, Psilura, Steph.

539 Detrita, Esp., H., O., G., B..... Germ. bor.

540 Rubea, F., H., O., G., B.. ..... \{ $\begin{aligned} & \text { Austria } \\ & \text { Gallia, Carnoti. }\end{aligned}$

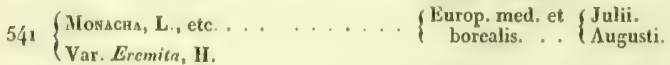

542 Dispall, $\mathbf{L}_{n,}$, etc. . . . . . . . Europ. ... Junii.

543 v. Arlantica (1), Ramb. Faun. And. . . $\begin{aligned} & \text { Hispan. mer. } \\ & \text { Sardinia. }\end{aligned}$

(1) Mabital qุoque ad Alger. 
tt† Alx nivex ano concolori. G. Leucoma, Stepls.

544 Sazıcss, L., etc. . . . . . . . Europ. . . Julii.

††ナ† Alæ nivex, ano luteo. G. Porthesia, Steph.

545 Agrtelda, F., H., 0,, G., B. . . . E. Europ. . . . J Julii.

$540^{\circ}$ Chrysonrhes, L., etc. . . . . . . Europ. .... Julii.

Notd. Arcures Sparshallii, Curt. 244. Habitat Americam, et erronee ut species Anglica adseriptus. Colore et statura magnam aflinitatem cum $\boldsymbol{L}$. Silicis et Chrysorrheen prebet.

2. GENUS ORGYA, Boisd., Ochs.

Bombyces, Dasychira, Demat, Orgya et Latia, Steph.

Larrx elongatx, tuberculatx, sctosim pilos $x$, scopulis dorsalibus, penicillisque antennx- et caudx-formibus ornatx. Puppa follicnlat $x$ - $-\Lambda n$ tenna sat breves, maris valde, feminx obsoletius pectinatx. Lingua vix ulla. Statura maris sxpias gracilis, femine crassior, obesa; hic alatus, illa síppius aptera.

+ Feminx alatx.

- Alre albida dorso lævi. G, Lalin et Leucomn, Steph.

$5\left\{\begin{array}{l}\text { V. Nigrum, F, O., G., B. . . . . E Europ. cent. . Julii. } \\ \text { Nivosa, H. }\end{array}\right.$

5 /8 Cenosa, H., Curtis., B., Tr., Frey., D. $\left\{\begin{array}{l}\text { Angtia. } \\ \text { Neustria. } \\ \text { Gall. bor }\end{array}\right.$

* Alæ transversion strigatx, dorso cristato. G. Dasychira et Demas, Steph.

$549\left\{\begin{array}{l}\text { Pudinunds, L., etc. . . . . . . . Europ. .... Maii. } \\ \text { Juglandis, H. }\end{array}\right.$

550 Arietrs, Esp., H., O., B. . . . . . . Germ. mer.

55. $\left\{\begin{array}{l}\text { Fascluna, L.. etc. . . . . . . Europ. cent. . \{ Junii. } \\ \text { Medicaginis, II. }\end{array}\right.$ 


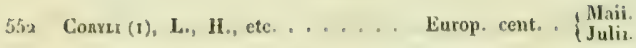

553 Serenetica, Esp., Q., G., B...... German. Lathyri, II.

t† Feminæ apter $x$.

554 Gonostigra, F., H., O., G., B... $\begin{aligned} & \text { Gallia..... } \begin{array}{l}\text { Junii. } \\ \text { Septembr. }\end{array} \\ & \text { German. }\end{aligned}$

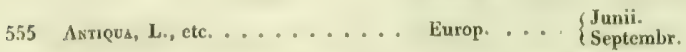

$556\left\{\begin{array}{l}\text { Encre, Germ., B., Tr. . . . . . Saxonia ... } \\ \text { Antiquoides, Heyer, IX. }\end{array}\right.$

557 Ropestres, Ramb., B., D ...... Corsica.

558 Tracotephas, B., D., Lefebv. . . . Galloprov.

Sicilia.

559 Corsica, B. Iconcs. . . . . . . \{ $\begin{aligned} & \text { Sicilia. } \\ & \text { Corsica }\end{aligned}$

560 - v. Aunolimaita, de Villiers, D., B. . . Pyren.

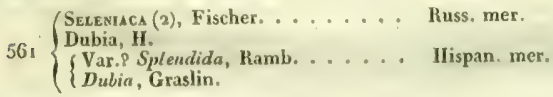

\section{GENUS CLIDIA, Boisd.}

Larvæ sub-gregariæ, sub-tuberculato-pilosæe. Puppæ folliculatæ. - Antennx longiores, maris crassc dentatæ, feminæ ciliatæ. Palpi recti, apice distantes. Lingua brevissima. Statura parva, robustior, noctuxformis; femina crassior. - Volatus nocturnus.

$56.2\left\{\begin{array}{l}\text { Geographica, F., D., B....... } \\ \text { Sericina, H. } \\ \text { Austera, Esp. }\end{array}\right.$

(1) Bomb. Coryli Auctorum a congeneribus discrepat antennis sub-longioribus, minus pectinatis, sed alìis characteribus, tum larva q̨uam imagine desumptis, plane cum Orgyis conveoit. Celeb. Stephens et Curtis genus proprium sub nomine Demes instituerunt.

(2) In speciminibus e Tagaorok et Sarepta Russize ale antica pallideflava; in individuis llispanicis contra, alos antice vivide fulva ut postiço. An opecie diversa? 


\section{TRIBUS BOMBYCINI.}

Larvx solitares, aut gregarix, haud tuberculatie, vel vix tuberculatopilose, lateribus densius pilos $x$.- $-\mathrm{Al} x$ deflexa, reversæe. Antenuæ maris valde, femina obsoletius pectinatæ. Statura robustior. Abdornen feminarum crassum, obesum.

\section{GENUS BOMBYX, Boisd.}

Bombyces, Auct. Gastropache, Ochs. Lasiocampa, Trichium, Pocilocampa, Eriograster, Cnethocampa, et Clisiocampa, Steph.

Iarvx modo inordinatim pilosx, modo pilis sub-fasciculatis vestita - Lingua nulla. Palpi villosi, brevissimi. Thorax villosissimus, globulosus. Ala anticx, stigmate discoidali fasciaque repanda signata Abdomen feminarum sxpe lanatum.

+ Larva gregarix, caruleo fulvoque vittata. Folliculum laxum, pulvere sulphureo adspersum. Femina ova annulatim ordinantes. G. Clisiocampa, Steph.

563 Nedstrara, L. etc......... Europ. . . Julii.

564 Castrensis, L., etc. ........ P...... Julii.

565 Franconica, F., Esp., O., G., B. ... $\begin{aligned} & \text { Gallia mer. . Julii. } \\ & \text { German. }\end{aligned}$

t+ Larvæ gregarix. Folliculum oviforme. Abdomen feminarum Jana densa, cinerea indutum. G. Ériogaster, Germar.

566 Lanestris, L., etc........... $\begin{aligned} & \text { Maii. } \\ & \text { Septembr. } \\ & \text { German. }\end{aligned}$

$567\left\{\begin{array}{l}\text { Evensa, F., H., O, G., B. ...... } \\ \text { Lentipes, Esp }\end{array}\right.$

568 Catax, L., ctc.......... Gallia or. . (Maii. 
Ht Larva juveniles, gregaria. Folliculum oviforme. Abdomen feminx apice denudatum.

569 Lots, H., Ochs., Ramb. . ...... Hispan. mer.

†††† Larva processionex pilis urentibus. Abdomen femina apice lanatum. G. Cuethocrmpa, Steph.

- Metamorphosis subterranea.

$570\left\{\begin{array}{l}\text { Neogeas, Fisch, B. . . . . . . . R } \\ \text { Var. Herculeana, Ramb. Faun. And. }\end{array}\right.$

575 Pitrocampa, F, HI , O., G., B. ... Gall. mer. . Julii.

571 Var. Pinivorn, Kulv. (multo minor). Germ, bor.

57 a Solitaris $(8) \ldots \ldots . . .$. Tarcia Europ.

- Metamorphosis sub tela communi

573 Processionea, L. . . . . . . . E Europ. .... Julii.

$+t+\dagger+$ Larva juveniles, gregaria. Folliculum ovatum. Abdomen feminx sub-lanaturn, G. Trichiura, Steph.

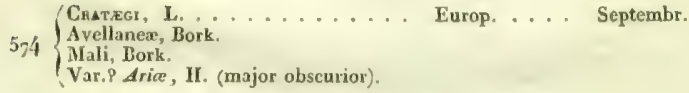

575 v.Incis, Ramb., Faun. And. . . . Hispan. mer.

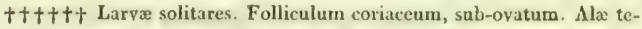
nue squamatæ. G. Poecilocampa, Steph.

576 Popol, L. . . . . . . . . Europ. .... Octobris.

$\dagger+†+†+$ Larvæ solitares, obesa, pilis rariusculis maculisque nigris, bi-seriatis. Mctamorphosis subterranea absque folliculo. Puppe apiec bi-mucronatæ: - Volatus maris diurnus. G. Lasiocampa, Steph.

$5_{77}$ Domets, L., etc. . . . . . . . . Europ. cent. . $\left\{\begin{array}{l}\text { Septembr. } \\ \text { Octobr. }\end{array}\right.$

5,8 Taraxaci, F., Esp., H., O., G., B. . \{ $\begin{aligned} & \text { Gall. or. . . Octobr. } \\ & \text { German. }\end{aligned}$

(1) Inter Pityocampam et Processioneam media. 
$+t+t+t+\dagger$ Larvæ solitares, pilis brevioribus, sub-intricatis, densis vestitx. Volatus maris diurnus. Antemna late pectinate. G. Lrsiocampa, Steph.

- Folliculum laxum, fusiforme.

Rubr, L., etc. . . . . . . . . Europ. . . . Maii.

\section{- Folliculum coriaceum, glandiforme.}

58 Sparti, H., Tr., B., Frey. . . . . . Europ austral. Junii.

58 I Qeercus, L., etc. . . . . . . . . Europ. .... Julii.

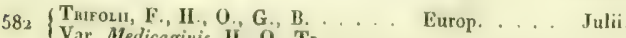

Var. Medicrginis, II., O., Tr.

583 Cocers, H., D. Tr. (an var. preced.P), Sicilia.

\section{GENUS ODONESTIS, Germar. \\ Lasiocampa, Boisd. olim. Gastropacha, Ochs.}

Larva pilosa, graminivora, antice posticeque penicillata, ctorso beeriter tenueque fasciculato; lateribus pilis reclinatis dense vestitis. Folliculum fusiformc.-Antenna late pectiuatæ. Palpi rostriformes, producti, valde pilosi Alie denticulatx, stigmate discoïdali fasciaque repanda signatx. Thorax globulosus, valde pilosus.

$584\left\{\begin{array}{l}\text { 'отлтонı, L., ete. . . . . . . . Europ. bor. . . } \\ \text { Var. A. (femina maris colore). }\end{array}\right.$

3. GEIYUS LASIOCAMPA, Latr., Boisd, Dup.

Gastropachae, Ochs., Steph. Bombyces, Auct.

Larve depress $x$, arboricol $x$, collares, pilosulx, dorso nudiusculo, sæpius tuberculigero; lateribus pilosis appendiculis mamillaribus, pediformibus, hirsutis, preditis. Folliculum sacciforme.-Antenna angustioses, pectinatx, post mortem sub-contortx. Palpi sxpius rostriformes, producti, dense squamato-pilosi. Alwe plus minusve denticulata, stigmate discoïdali fasciaque repanda signate.

+ Als latiores:

585 Pixt, L., etc. .......... Gall, mer. etc. 
586 Prusi, L., etc. . . . . . . . Europ. cent. . Junii.

$587\left\{\begin{array}{l}\text { Quencifolsa, L., etc. ....... Europ. .... Julii. } \\ \text { Var. Ulmifolin, Dah.. } \\ \text { Var. Alnifolia, Ochs. }\end{array}\right.$

588 Poporfouta, I., II., O., G., B... \{ Gall. bor. . Junii

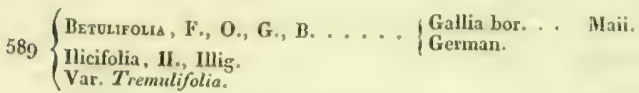

$590\left\{\begin{array}{l}\text { Iucrrous, L., O., G., B. . . . . . } \\ \text { Betulifolia, Esp., H., Illig. }\end{array}\right.$

5gy v. Suberifolia, Ramb., Faun, And. Hispan, mer.

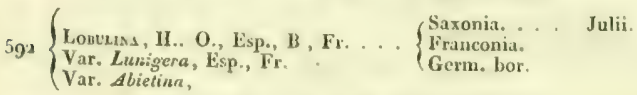

$+t$ Alx clongatx, angustiores.

593 Lineosa, B., Tr., D., Fr., Il. . . . Gall. austral. Junii.

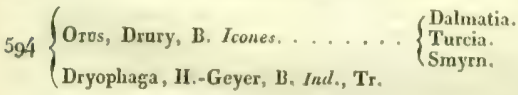

\section{GENUS MEGASOMA, Boisd., Dup. \\ Gestropacha, Tr.}

Larvx collares, cylindricx, elongat x, pilosulx; dorso nudiusculo, tuberculis minutis pilosulis instructo ; lateribus tuberculis pilosis longioribus. Folliculum fusiforme, laxum. P'uppa pilis fasciculatis induta. - Antenix maris ad basin valide pectinatx, dein semipectinata et sub-convoluto-sinuatx. Palpi hirsuti, sub-rostriformes. Abdonen solito longius. Ale antica sinuate, stignate discoüdali fusriaque repanda signatx. - Species hujus generis, sequenti excepta . Africam inhahitant.

$595\left\{\begin{array}{l}\text { Repandum, B., Feisth.., D. . . . . Hispan. mes } \\ \text { Repanda, II., Tr., Fr. }\end{array}\right.$ 


\section{TRIBUS SATURNIDES, Boisd.}

Larva ohesx, arboricolx, secmentis prominulis; modo tuberculis piligeris, modo spinis verticillatis, vel peunatis instructa. Folliculum tenax.-Aln patulx, lat $x$, sapius macula ocellari vel diaphana ornat $x$. Lingua nulla.

\section{GENUS SATURNIA, Schranck, Ochs., Boisd.} Ateacus, Germ., Latr.

Larva pilis rigidis, rariusculis, radiantibus, tuberculatis.-Corpus villosissimum Antennæ breviores, maris late pennatx, feminæ pectinat Palpi breves, villosissimi. Thorax rotundatus, collari uti costa alarum colorato, praditus. $\Lambda$ bdomen obesum, brevius. $\mathrm{Al} x$ lat $x$, patula, modo macula ocellari, modo macula chiaphana ornatie (qux macula semper nervulo distincto secta).

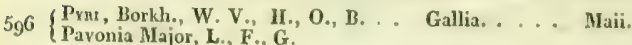

(Pavonia Media, F., Esp., G.

(1) S. Carpini in Germadia cum S. Spini copulans, secundum Dom. Treitschle, hatud senet obvia, ende varictates hybrido. 


\section{$\mathrm{XX}$. TRIBUS ENDROMIDES.}

Larvx glabra, arboricol $x$, sphingiformes, mutic $x$ vel spinigera. Folliculum laxum, sericeum, aut vix ullum. P'upp sxpius quisquiliis vel terra sepultx- - Antenna naris pectinata vel pennata. Ala sub-deflexx, patulx, macula seu stigmate discoïdali signatx.

Ad hanc tribum attinent genus Cerocampa ex $\Lambda$ merica, genusque $S_{e-}$ ricaria ( $\boldsymbol{B}$, Mori, $\Lambda$ uct.) ex $\Lambda$ sia.

\section{GEIVUS AGLIA, Ochs., Boisd. \\ Bomilyx, Auct.}

Larvæ rugulosæ, per juventutem spinigerx; adultæ muticx. Folliculum sub-nullum. Puppa muscis vel foliis demortuis obtecta. - Antennx breviores, maris pennatx, femina pectinatx. Palpi villosi, breves. Lingua nulla. Ablomen obesum, breve. Ala raacula discoildali ornata. - Volatus maris sub-meridianus.

Ad hoc genus referenda Bomb. Erythrina, Fab., e Guyana.

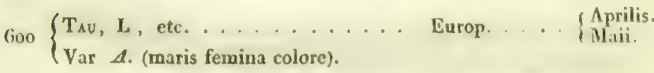

\section{GENUS EIVDROMIS, Ochs., Boisd. Dorvillin, Leach. Bomby.r, Auct.}

Larra lavis, glaberrima, sphingiformis, segmento anali incrassato. Folliculum laxum, sub muscis occultatum.-Caput parrum, retractum. Antennx apice obtusx, anguste pectinat $x$, feminx vix graciliures. Palpi breves, pilosissimi. Alx sub-deflexx, strigis transversis stigmateque discoïdali signatx. - Volatus maris meridianus.

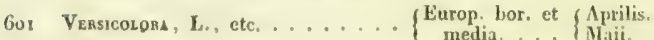




\section{TRIBUS ZEUZERIDES, Boisd.}

Larva elongate, scutellatx, decolorat $x$, in radicibus, fruticibus vel truncis occulte degentes, pilis rariusculis adspersa. Puppa elongata segmentis scabris.-Corpus villosum, vel piloso-squamatum. Lingua vix ulla. Antenna modo brevissimx, modlu longiores, filiformes vel sub-pectinata. Abelomen clongatum, femina: sxpius oviducto exserto præditum. - Volatus gravis, nocturnus.

1. GEINUS COSSUS, Boisd.

Cossi, Latr., Fab.

Larva elongata, subtus depressa mandibulis validis.-Corpus crassum, squamosum. Thorax rotundatus. Antennæe maris modice pectinate, femine dentata. Alx deflexw, tectiformes, nebulusx, cinereo alhidoque tessellato-strigulate. Abdomen elongatum, obesum, femina oviducto exserto.

Go2 $\left\{\begin{array}{l}\text { Ligniperda, F., O., G., B. . . . . Europ. . . . . Julii. } \\ \text { Bomb, Cossus, L., Esp., H. }\end{array}\right.$

603 v.Temebra, F., II., Esp., Ochs. . . . . German. ... Julii.

Go' Coesthuss, H. O., B. Gall. mer, . Julii.

$\left\{\begin{array}{l}\text { Coesthum, H., O., B. } \\ \text { Var. Teredo, Boisd. Index. }\end{array}\right.$

$605\left\{\begin{array}{l}\text { Tursps, H., B. . . . . . . Russ. mer. } \\ \text { Kindermannii, Frey. }\end{array}\right.$

3. GENUS ZEUZERA, Latr., Boisd.

Cossi, Ochs., Fab.

larva et puppa at jam dicte-Corpus crassum,squamato-tomentosum. Thorax rotundatus. Antenne maris basi pectinata, apice setiformes, femina obsoletius pectinata, rel tantum basi tomentosa. $\Lambda$ le unicolores vel punctate. Abdomen clongatum, femina oviducto exserto 
+ Alx punctatx.

GoG fiscurr, L., etc.......... Europ. . . Julii. Var. P Octopunctala, B. Icones (1). Sicilia.

t† $\Lambda$ lx unicolores (an genus proprium?).

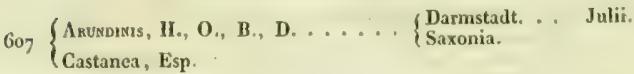

3. GeNus ENDAGRia, Boist.

Cossus, Ochs.

Larva elongatx, radicivorx.-Corpus debilius, villosum. Antenne? sat clongate : maris unidentatr, femine ciliate. Palpi squamoso-hirsuti. Alæ breviores, latiusculx, cinereo albidoque variegatx. Abdomen gracilius, feminxe oviducto cxserto.

$608\left\{\begin{array}{l}\text { PAntuenasa (2), Boisd., II. ...... } \\ \text { Coss. Panthcrinus, Oclis. } \\ \text { Ulula, Esp., Borkh. }\end{array}\right.$

\section{GenUS IIEPIALUS, Fab., Latr.}

Hepiolus, Ochs., Illig.

Larva: radicivore, graciles, elongates. - Corpus debilius, elongatum, villosum. Antenna in nostratibus brevissima (in pluribus exoticis ordinaria), moniliformes, vel dentata. Palpi hrevissimi, hirsuti. Ala omnes elongatx, lanceolatx, tenues. Abdomen gracilius.

+ Uterque sexus alatus.

Gon Hurou, L., etc. . . . . . . E Europ. bor. Junii.

610 Velleda, II., Esp., Ochs. ...... Alpes. . ... Julii.

(1) Minima, statura $Z$. Filipendula; thorace punctis ceruleis octo.

(2) Specimina e Gallin australiori proserliraque ox IIspaoia valde robustiora, $2 x$ pius Coss. Castrum acquad. 
Cannus, li., O., B............... Jlpes. Julii.

611 $\left\{\begin{array}{l}\text { Carna, II. } \\ \text { Var. Jodutua, II. }\end{array}\right.$

6r2 $\left\{\begin{array}{l}\text { Sxuvinus, L., Esp*, O., G., B. .... Europ. . . . . } \\ \text { Lupulina, II. }\end{array}\right.$ Vax. IIamma, II.

Var. Flinu, Jisp.

613 Gasma, H., O., B. . . . . . . . Alp. hely. . Julii.

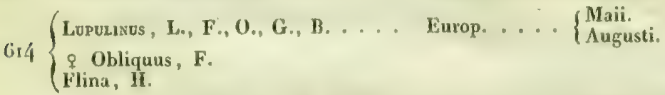

$615\left\{\begin{array}{l}\text { Hectus, L., F., O., G., H., B. . . Europ. . . . Junii. } \\ \text { Var. Nemorosa, Esp. }\end{array}\right.$

t+ Fcmina sub-aptera.

616 v.Pyrenaicus (1), Donzel, Ann. S. Ent. Pyren. . . J Julii.

(1) Statura H. Sylvine; alse aotice fuacre, punctis numerosis, inaqualibus, llavido-albidis adsperse: postica fusco. 


\section{TRIBUS PSYCIIIDES, Boisd.}

Larve vaginis e quisquiliis inclus $x$, vaginasque sccum protrahentes. Metamorphosis in vagina. - Lingua brevis. Corpus villosun. Ala deflexæ, tenuiter squamatæ. Statura parva.-Volatus maris diurnus.

Hac tribus cum Tinetidibus victu habituque listrarum valde congruit, sed insecto perfecto plane ad Bombyces Auctorum accedit.

Iluc tribule genus $O E$ ceticus Americes referendnra.

\section{GENUS TYPHONI \\ Chimcera, Ochs., Boisd. olim.}

P'alpi brevissimi, sub-nulli. Antennæ maris acutx, ad basin crassiorcs, articulis singulis pilis squamosis instructis. Antennx femina setiformes, sub-pilosulæ. Thorax squamato-pilosus. Ala clongata. Abdomen maris apice fasciculatum, feminæ oviducto exserto.

$617\left\{\begin{array}{l}\text { Logubus, O., B. . . . . . . . } \\ \text { B., Lugubris, H. } 217 .\end{array}\right.$

Gis Melas, Boisd. (1)........ \{ $\begin{aligned} & \text { Pyren. .... Julii. } \\ & \text { Alp. Prov. }\end{aligned}$

a. GENUS PSYCIIE, Schranck, Ochs., Latr., Boisd.

Psyche et Fumea, Steph.

Larve glaberrimx, postice vermiformes, decolorate, antice pallide lutex, nigro strigatx, - Mas : Antennæ breviores, pectinatæ vel plumosa. Corpus valde pilosum. Palpi breves. Alx tenuissime squamatxe, vel sub-diaphanx. Femina : Aptera vel sub-vermiformis, vagina inclusa copulam vitamque consumens.

Nota. Psychicles manipuli primi vix cum aliis conveniunt, et aliquando forsan, cum melius observatx fuerint, ad Tineides referendæe.

(1) Differt a Typhonia Lugubri, fimbriis alarum nigris antennisque manifeate crassioribus. 
t.Intenn. pertinut. corp. debilius. Feminx apterx, pedibus antennisque complet. G. Fumea, Steph

6 เ9 $\left\{\begin{array}{l}\text { Purl } A, \text { Esp., O., B. . . . . . } \\ \text { Plumella, II., W. V. }\end{array}\right.$

Gzo v. Plumella, 0., B........ $\begin{aligned} & \text { Austria. ... Julii. } \\ & \text { Gallia }\end{aligned}$

621 Nurmella, II., O., G., B. ...... Gallia. .... Junii.

6ar Radierla, Curt. $332, \ldots \ldots . .$. . . .... Junii.

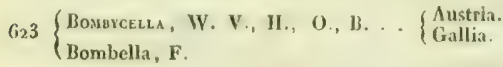

6.24 v.Pectunela, F., H., W. V., O., B. . Gallia..... Julii.

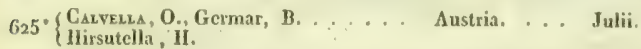

Ga6 Nudelua, 0., B. .......... Grallia...... Augusti.

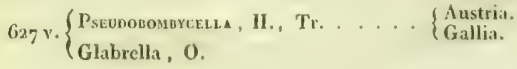

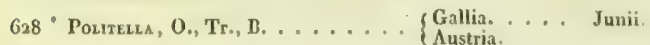

629 Mugnerza (s). . . . . . Monspelii.

it Antennx plumos. corp. crassius. Feminx vermiformes.

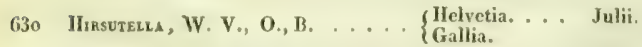

631 $\left\{\begin{array}{l}\text { Muscella, F. W. V., H. ..... } \\ \text { Graminella, Vieweg. }\end{array}\right.$

63a Pronirena, 0., B......... Pyren. ... Julii.

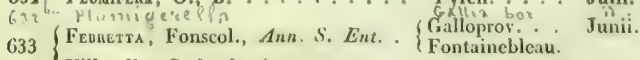

Villosella, God. pl. 24.

634 y.Gandiella, Anderregg (2)..... Valesia.

(1) Magnitudo Viciella : ulac cinerue frimbria obscuriori, lucida ; antennæ breviuscule.

(8) Mari 8 . Franconice eqquatis et sub-siroilis; alis robustis, fusco-lutescentibus. An gen. proprium ? 
$635\left\{\begin{array}{l}\text { Amronsıs, Rossi, F., Esp., O., G., B. }\left\{\begin{array}{l}\text { Gallia. . . . Julii. } \\ \text { Italia. }\end{array}\right. \\ \text { Fucella, H. }\end{array}\right.$

636 Peruoselza, Ramb., Fann. And: . . Hisp. mer.

$637\left\{\begin{array}{l}\text { Arbida, Esp., B., Tr. ....... } \\ \text { Vitrella, H. } \\ \text { Semiluctifera, Devill. }\end{array}\right.$

638 Bicolokella (1).......... Gall. mer.

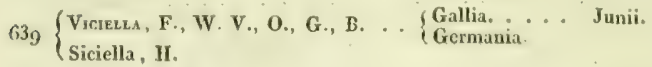

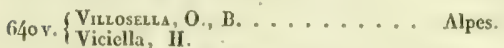

6\}s $\left\{\begin{array}{l}\text { Graminella, W. V., H., O., B. . . Europ. ..... Junii. } \\ \text { Vestita, F. }\end{array}\right.$

(1) Statura Plumifere : alo pallide nigro-fuscs, omnium basi late fulvo-lutescente. 


\title{
XXIII. TRIBUS COCLIOPODES, Boisd.
}

\author{
Arcliida, Steph.
}

Larva singulares, depress $x$, abbreviata, limaciformes, scutelliformes, vel testudiniformes, vix alias erucas referentes, mutica vel lateraliter appendiculatx, glabre vel sub-pilosulx. - Lingua brevissima. Antennx maris sub-pectinatre vel sub-dentato-filiformes. Alæ deflex $x$. Statura media vel parva.

Insecta hujus tribus in Europa paucissima, frequentia vero in America septentrionali.

\section{GENUS LIMLCODES, Latr., Boisd., Steph}

Ileterogenea, Knoch, Treits.

Larvæ scutelliformes, pedibus membranaceis, sub-nullis. Folliculum membranaceum, oviforme, vel globuloso-ovatum. - Antennæ longiores, maris crassiores, dentatx; feminx filiformes vel ciliatæ. Lingua sub-nulla. Palpi breves, recti, contigui, minime ascendentes, fronte vix longiores. Corpus sat robustum, pilosum. Ala breves, latiores ac robustiores.

$6 .\left\{\begin{array}{l}\text { Aserzus, F., G., B......... } \\ \text { Asella, Borkh. } \\ \text { Tortr. Asellana, II. }\end{array}\right.$

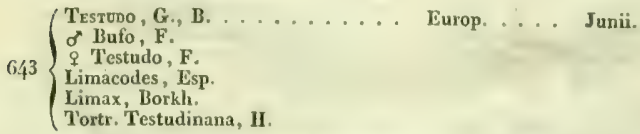

Notre. Dux tantum species ex Europa, ac circiter quadraginta ex civitatibus foederatis Americes numerantur; quarum alix ad Bombyces et alixe ad Platypteryges prope accedunt. 


\title{
XXIV. TRIBUS DREPANULIDES, Boisd.
}

Larva arboricolx, postice attenuate, pedibus quatuordecim tantum instructæ; pedibus analibus in spinulis didymis, supinis conversis, ano rarius apodo, mutico. Puppa inter folia folliculata. - Als deflexæ, sæpius patulæ. Habitus Geometriformis.

\author{
ז. GENUS CILIX, Leach. \\ Platypteryx, Tr., Boisd. olim, Latr.
}

Antenna maris pectinat $x$, feminx fere filiformes. $\Lambda$ la integra, acute tectiformes; anticx apice rotundatx, absque fasciis repandis. Palpi brevissimi. Lingua vix ulla. Statura gracilis.

$644\left\{\begin{array}{l}\text { Spineu, H., Lasp., Tr......... } \\ \text { B. Compressa, F., Esp. } \\ \text { Ph. Att. Iluffa, L. }\end{array}\right.$

a. GENUS PLATYPTERYX, Lasp., Boisd, Leach.

Drepana, Schrank.

Antenna maris pectinatx, feminæ ciliatæ. Statura debilis Geometrarum. Alæ patulx, horizontales ; anticæ apice mucronato-falcatx, stigmato discoidali faseiisque repandis signatx. Palpi breves. Lingua sat elongata.

† Alx anticx dentatx. G. Platypteryx, Steph.

$645\left\{\begin{array}{l}\text { Lacertura, H., Esp., Lasp., Tr. . Europ. .... } \\ \text { Geom. Lacertinaria, L. } \\ \text { Var. Scincula, H. }\end{array}\right.$

tt Alx anticx integrax. G. Drepann, Steph.

$646\left\{\begin{array}{l}\text { Siren , II , W'. V., Lasp., Tr. ... } \\ \text { Ilarpagula, Lisp. } \\ \text { Ml. Harparia, F. }\end{array}\right.$ 
DIRPANURIOES .

8:3

$6 .\left\{\begin{array}{l}\text { Cunvarura, Lasp., Bork., Tr .... } \\ \text { llarpagula, II. }\end{array}\right.$

$6\left\{\begin{array}{l}\text { Fatcou }, \text { H. Esp, Lasp., Tr. .... } \\ \text { Geom. Falcataria, L., F. }\end{array}\right.$

6.99 $\left\{\begin{array}{l}\mathrm{IH}_{\text {andra }}, \text { Esp., II., Lasp., Tr. ... } \\ \text { Ph. Falcata, F. } \\ \text { Var Uncula }, \text { H. }\end{array}\right.$

$650\left\{\begin{array}{l}\text { UxGucula }, \text { H., Lasp., Tr. ..... } \\ \begin{array}{l}\text { Sicula, Esp., Bork. } \\ \text { Cultraria, F. }\end{array}\end{array}\right.$ 


\section{TRIBUS NOTODONTIDLS.}

Pseudobombycini, Boist., olim. Bombyces, Auct, vet.

Larvæ arboricolæ, glabrx, vel pilis rariusculis adspersæ. Metamorphosis sapius hypogan, rarius inter folia arborum vel in folliculo membrenaceo. - - ntennx maris pectinatx. Lingua breviuscula, conspicua. Alre frenatæ, plane tectiformes. - Volatus nocturnus.

1. GENUS DICRANUHA, Latr., Boisd.

Cerura, Schrank, Steph. Harpya, Oclis, Pania, Dalm.

Larva glaberrima, pedibus tantum quatuordecim instructar, postice attenuatr; segmento anali in tentacula duo desinente; virides, dorso pallio obscuriori tectx. P'uppa folliculo membranaceo ad arborum truncos inclusa. - Antennx in utroque sexu pectinatx, post mortem intus contorta.

651 Venoasct, G., B. . . . . Monspelii. . . Maii.

652 $\left\{\begin{array}{l}\text { Brcusprs, H., B., O. ....... } \\ \text { Furenla var. B. Zudex. }\end{array}\right.$

Var. Integra, Steplt.

$653\left\{\begin{array}{l}\text { Bifida, II, O, B. (Parisiis sat frequens.) Gallia. . . . Maii. } \\ \text { Julii. }\end{array}\right.$

Furcula var. B. Index.

654 Fuscrouk, H.(ansat a praced.distinct.8) Russia..... Maii. Forficula, Fisch.

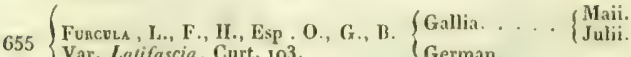
$\left\{\begin{array}{l}\text { Var. Latifascin, Curt. 193. } \\ \text { Var.P Uracera (obscurior). . . . . Merman. }\end{array}\right.$

656. Ermsea, Esp., H., O., G., B .... Grallia.... Maii.

657 Visura, L., F., H., O., G., B. ... Europ. .... Maii. Var. Minax, H.

658 v.Puntosa, Dalm. (1)........ Lapponia.

(1) Statura et habitus Vinute: alis nigricantibus immaculatis; corpore albo, aupra nigro punctato; abdominis dorso nigro segonentis margine albis. Habitat in salicibus Lapponiz et Suecix borralis. 
2. GENUS IIARPYIA, Ochs, Boisd.

Stauropus, Steph.

Larva glabrx, gibbosa, terrifica, pedibus tantum quatuordecim instructæ; segmento ultimo apodo, truncato, supino. - Puppa folliculata. Antenna maris pectinatx, apice nuda, setiformes. Lingua brevis, conspicua.

$+$

$659 F_{\Delta G 1}$, L. etc. ......... G Gall. bor. . Junii.

$+t$

$660\left\{\begin{array}{l}\text { Mrumesear , F, O., Lsp., G., B. . . \{ Gallia. .... Junii. } \\ \text { Termanica, H., W, V., Bork. }\end{array}\right.$

3. GEIVUS UROPUS, Rambur., Boisd.

Urapyia, Boisd. olim, Ochs.

Jarva sub-glabra, elongata, postice attenuata ; segmento anali tentaculis duobus elongatis, prehensilibus instructo. Puppa hypogxa. - Antenææ maris pectinatæ, apice nudæ, setiformes.

$661\left\{\begin{array}{l}\text { Uum, Bork, 1I., O., D.. B. .... } \begin{array}{l}\text { Austria. ... } \\ \text { Cassinia, Esp. }\end{array} \text { Mall. mer. }\end{array}\right.$

4. Genus asteroscopus, Boisd, Treits.

Petria, Steph.

Larve glaberrima, subdiales, sub-sphingiformes, segmento penultimo incrassato, capite per quietem supino. R'uppa liypogara.-Antennz: Iongiores, maris pectinat $x$, femina dentato-crenulat $x$. $\Lambda$ la elongat $x$ fuscostrigosæ。

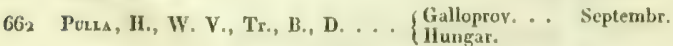

$663\left\{\begin{array}{l}\text { Cassina, F., II., IV. V., Tr., B., D. \{ Gallia..... Novembr. } \\ \text { German. }\end{array}\right.$ Sphinx, Esp. 


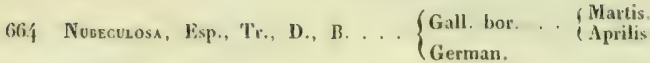

\section{GENUS PTILODONTIS, Steph.}

Orthorhinia, Boisd. olim. Notodonta, Ochs.

Larva lavis, depressa, utrinque attenuata. Puppa hypogaa. - Antenna maris pectinata. Palpi recti, lite piloso-squamosi, horizontales, solito multo longiores. $\Lambda \mathrm{l} x$ anticx ad marginem internum lobulo dentiformi instructæ.

Palpina, Lo, etc. ........ Europa. . . . $\begin{aligned} & \text { Maii. } \\ & \text { Julii. }\end{aligned}$

6. GENUS NOTODONTA, Ochs., Boisd.

Bombyces, Auct. Notodonta, Leiocampa, Lophopteryx, Ptilophora, Chaonia et Peridea, Stepl.

Larva glabre, vel pilis rarissimis indute ; modo dorso gibbosa; modo segnento penultimo incrassatar vel bicuspidatx; sxpius laves, unitxe. Puppa terra vel quisruiliis sepultx. - Antenua sat elongat $x$, maris pectinatix, feminx dentata. Ale antice sxpius appendiculo dentiformi margine interno instructæ.

t Larrx segmento penultimo bicuspidate. G. Lophoptery $x$, Steph.

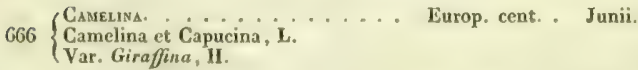

$6 G_{7}\left\{\begin{array}{l}\text { Cuculırs }, \text { W. V., H., O.. G., B. . } \\ \text { Cuculla, Esp., Borkh. }\end{array}\right.$

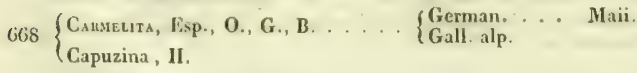

H Larva segmento penultimo incrassato, G. Leiocampa, Steph.

66 Dicres, 1., ete: ......... Europ. ... \{ Maii. 
670 Dicteones, Esp., II., O., G., B. . Europ.... Julii.

Gnoma, F.

tt+ Larvæ dorso gibbosæ. G. Notodonta, Steph.

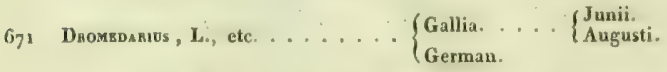

$6_{72}\left\{\begin{array}{l}\text { Trumopitus, F., Esp., O., G., H. (text.) } \\ \text { Torva, HI. 27. W. V. } \\ \text { Tremula, I1., W. V. }\end{array}\right.$

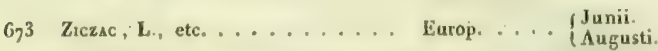
$674\left\{\begin{array}{l}\text { Tonva, O., G., H. (tezt)....... Gall, bor., etc. Maii. } \\ \text { Tritophus, H. 29. }\end{array}\right.$

t+t Larvx læves, unitx.

"Perider, Steph. $675\left(\begin{array}{l}\text { Tneptos, Fi, Esp., O., G., B. . . . Gall., etc. .. Maii. } \\ \text { Tremula, W. V., H. }\end{array}\right.$

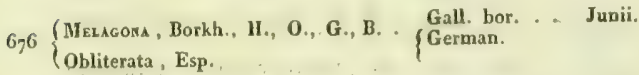
$677\left\{\begin{array}{l}\text { Veuraris, Esp., O., G., B. . . . G. Gall, etc.. Junii. } \\ \text { Austera; H. }\end{array}\right.$

6-8 Brcolora, F., H., O., G., B. . . . Gall. bor., etc. Junii.

679 v.AгвIDA,Zetter. (an a praceced.sat dist. P) Russia mer.

680 Angentinı, F., W. V., H., O., G., B. $\left\{\begin{array}{l}\text { German. } \\ \text { Gall. }\end{array}\right.$

\section{G. Chaonia, Steph.}

68ı Querna, W. V., F., H., O., G., B. Gall, occid. .. Junii.

$682\left\{\begin{array}{l}\text { Cusomis, H., W. V:, O., G., B. . : G Gall., etc. . \{ Aprilis. } \\ \text { Rohoris, F. Steph. }\end{array}\right.$ 
(Dodicis, F. W. V., H., O., G., B. . . Gall., etc. . Maii, Trimaculata, Esp.

Tripartita, Bork.

684 Hхвнıs, II., B., Ramb. . . . . . \{ $\begin{aligned} & \text { Hispan. } \\ & \text { Monspeli }\end{aligned}$ *... G. Ptilophora, Steph. (servandum forte).

$6 \$ 5$ P'xomerers, F, W. V., Esp., H., O, G. $\left\{\begin{array}{l}\text { Gall. bor. . Junii, } \\ \text { German. }\end{array}\right.$

\section{GEIVU GLUPHISIA, Boisd. Notodonta, Oclis.}

Larva lavis, depressa, glabra. Puppa inter folia sub-folliculata. $-\mathrm{An}$ tennæ maris late pectinatx. Palpi ordinarii. Alæ breves, latiusculx; anticx absque lobulo interno.

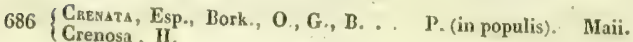

\section{GENUS DILOBA, Boisd. Ĺpisema, Ochs., Steph.}

Larva pigra, sulddialis, sub-pilosula. Puppa folliculo membranaceo inclusa. - Antennæ longiusculæe, maris pectinatæ, feminx dentate. Palpi ordinarii. Lingua spuria. Alæ latiusculæ, maculis duabus geminatis majoribus inscriptæ.

68 y CozndreocepHalı, I.., etc. . . . . Europ. . . . O Octobr.

9. GENUS PYG ERA, Boisd.

Pygrara, Ochs. Sericarice, Latr. Bombyces, Auct.

Larva flaccidx, elongatx, pilosiuscula, per jurentutem sub-gregarix, lineatz. Puppa liypogxa. - Antenme pectinatx. Caput retractum. Thorax crassus, rotundatus, collari ferrugineo. Ala elongate, Abdomen cylindricum, elongatum

688 Beczphara, L., etc. . . . . . Lutop. . . . M Manti.

689 Bocephazoldes, H., O., B. ..... \{ $\begin{aligned} & \text { Gall. mer. . . Junii. } \\ & \text { Ilungar. }\end{aligned}$ 
10. GENUS CLOSTERA, Hoffmansegg, Steph., Boisd. Pygare, Ochs. Sericario, Latr. Bombyces, Auct.

Larva pigra, crassiuscul $x$, pilosulx, inter folia alligata degentes, seg- . mentis quarto et ultimo sxpius tuberculatx. Puppa sub-folliculata. - Antennæ maris, feminæ tenuius, pectinatx. Alx latiusculæ, transversim strigosæ, corpore breviores. Abdomen resupinatum, maris apice penicillo bifido instructum.

Ggo Corturs, L, etc.......... Europa....

69i Arachoneta, F., Esp., H., O., G., B. Europa...... S Malii.

G92 Recuuss, F., Esp., H., O., G., B. . Europ. bor. . \{ Maii.

$6 g^{3}$ Arsstomosis, L., etc. . . . . . E Europ. bor. . Maii.

694 v. Trson, H., O., B., D. . . . . Russ. mer. . \{ Maii. 



\section{NOCTU无.}

Veterum auctorum Noctua, unum aliquid efficiunt, coharens illud atçue compactum, quod agerrime dividatur, seu pro basi statuantur metamorphoses, seu quis characteres ex insecto perfecto duxerit. Species autem in continuam seriem et plane natura congruentem digerere, majoris ctiam est diflicultatis, quum in his pracipue natura rectam lineam egressa, et irradiatione usa videatur.

Intra hos proximos annos, in Germania, in Gallia, in Anglia, multa earum Genera conflata sunt. Verum, paucis exceptis, nullo charactere illa constant qui certı fide stabilem se habeat; quare alia alii potius generi species adscribatur, non semper facile ratio occurrit. Itaq̨ue ea quie assumpsimus Genera, quibusque nonnulla nova addidimus (nihil enim est medium, aut plurima esse debent aut fere nulla), vero vocabulo tantum greges quos nominibus designavimus. Eas species conferre studuimus, apud quas similes cernuntur characteres, tum in primo earum habitu, tum postquam perfecta insecta evaserunt; denique ex qux vocavimus Geners sic disposuimus, nc quid absurdum esset, ita ut unumçuodque, quam maxime potest, superiori aut subsequenti contiguum existat. Posterius vero, quum in notitiam venerint metamorphoses quas patiuntur species exotica, fieri potest ut in magnum genus NOCT'UA Linnxi rursus aliqua parte inclinetur. Quod pariter Geometre sibi vindicent, alixque nonnulla familix, quas certis generibus dividere non magis in promptu esse judicamus. 


\section{TRIBUS NOCTUOBOMBYCINI, Boisd. Noctua Auctorum.}

Larva læves, arboricola, depress x, in folia alligata degentes. Puppa inteı folia laxe folliculata, rarius sub quisquiliis sepulta.-Antennæ maris crassiuscula. Lingua brevis crassior. Ala tectiformes. Abdonen haud cristatum.

\section{GENUS CYMATOPHORA (1), Treits, Boisd.}

Ceropacha, Steph.

Larva pallidx, deplanatx, capite colorato, latiusculo, cordato, plus minusve punctate. Puppa abbreviata. - Antenna mediocres, crassiuscula, sub-crenulatx. Thorax sub-lanatus, pterygodiis clevatis, et inde, in insecto vivo, antice bifidus dicendus. $A l x$ latiusculæ, undatæ, lineis transversis sat numerosis.

+ Corpore crassiori.
$695\left\{\begin{array}{l}\text { Runews, F., D., B. . . . . . . Gallia. . . . Aprilis. } \\ \text { Xanthoceros, H., Tr. }\end{array}\right.$
Erythrocephala, Esp.
Chrysoceras, Linu. Traus.

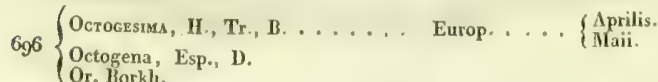
$G_{97}\left\{\begin{array}{l}\text { Or, F., H., Tr., B., D. ....... Europ. .... Maii. } \\ \text { Octogena, Esp. } \\ \text { Consobrina, Borklı. }\end{array}\right.$

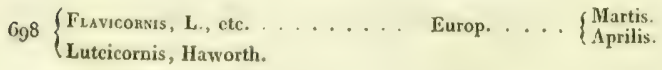

(1) Qui nomen illod geocris creavit D.Treitschke, rectius censuit pro $C$ poni $K$, et in supplemento suo scribiః Kymatophora. Neutrum D. Guéaée probavit, nec Cymafophora nec Kymatophora, verum pro utroque Chymatophora. Nostra autem opinione, non est cur in illa yocescribenda adsit dubitatio, quum derivetur grecis vocubulis $K \tilde{u} \mu \alpha$, unde, et $\phi_{E} \in \mathrm{f} \omega$, forro, et apud $\mathrm{L}$ atinos versio littera $\mathrm{K}$ in litteram $\mathrm{C}$ vulgaris fuerit; qquapropter scribebant $C_{y m b a} C_{y m b a l u m} C_{y}$ therea, etce, non $K$ ymba nee Chymba. Quid quod $\mathrm{K}$ in nullis verbis utendum putat Quintilianus, nisi qus sigoificat, etian ut sola ponatur?

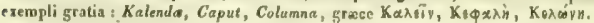


699 Dicuxa, F., H., Tr., D., B. . . . G Gall. bor. Augusti.

goo Ruficouns, F., W. V.. II., Tr, B. . $\left\{\begin{array}{l}\text { Austria. . . Aprilis. } \\ \text { Gall, or. }\end{array}\right.$ tt Corpore graciliore.

goi Fuvctuosa, H., Tr., B...... \{ $\begin{aligned} & \text { P., etc. .... Junii. } \\ & \text { Gall. bos. }\end{aligned}$

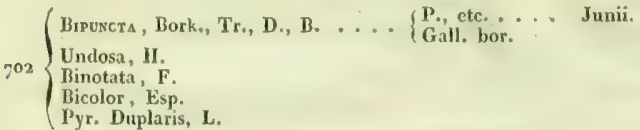

3. GENUS CLEOCERIS, Boisd.

Larvæ vix deplanatæ, antice attenuatæ, capite parvo, inter fascicula foliorumdegentes. Puppa sub quisquiliis tenuc folliculata -Antennx maris crenulato-sub-pectinatx, feminx tenuc dentatx. Thorax rotundatus.

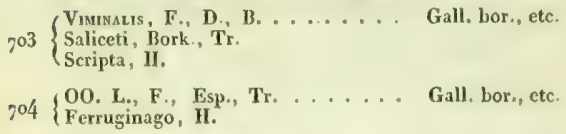

\section{GENUS PLASTENIS, Boisd.}

Tethea, Steph.

Larvæ virides, subtus depressa, postice attenuatx, capite parvo subglobuloso, inter folia degentes. Puppa vix folliculata, sxpius hypogæa.- Antennæ crassiusculæ, sat elongatæ, vix in mare sub-denticulatæ. Thorax rotundatus.

705 Sobrusa, F., W. V., II., Tr. ... Gallia, etc. . Julii.

7oG (Retusa, I.., etc. . . . . . Gallia, etc. Julii,

7o6 Chrysoglossa, Linn. Trans. 


\section{TRIBUS BOMBYCOIDES, Boisd.}

\section{Noctue Auctorum.}

Larva tuberculis piligeris instructe, plus minusve pilose, arboribus, herbis, vel lichenibus vescentes. Puppa folliculata. - Antennx in utroque sexu filiformes, rarius in maribus crenulate lingua sat producta. Ala tectiformes.

L.epidoptera hujus tribus Lipritides et Lithosias facie larvarum modoque vivendi revocant.

\section{GENUS ACRONYCTA, Ochs, Treits., Boisd. Apcteice et Acronyctae, Steph.}

Larva tuberculato-pilos $x$, pilis dorsalibus inordinatis vel fasciculatis. - Antennæ elongatæ in utroque sexu filiformes. Thorax levis, rotundatus. Abdomen lxve. Alx signo basilari pluribusque cuspidatis inscriptx. Macula rotunda, minuta; reniformi crassiori.

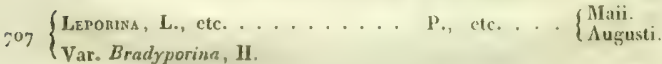

,08 $\left\{\begin{array}{l}\Lambda \text { ceris, L., etc. . . . . . . . P. P. etc. . . Junii. } \\ \text { Var. Candelisequa, Esp. }\end{array}\right.$

jo9 Megacephala, F., Tr., H., D., B. . P., ètc... . $\begin{aligned} & \text { Maii. } \\ & \text { Augusti }\end{aligned}$

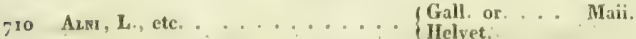

FI L Ligostr1, F., H., Tr., D., B. . . . P. ctc. . . Maii.

(Strigosa, F., Tr., D., B ...... Gall. bor. Junii.

712 Favillacea, II., Esp.

$713\left\{\begin{array}{l}\text { Trudens, F., Esp., Tr., D., B..... P, ctc. . . Maii. } \\ \text { Psi, II }\end{array}\right.$

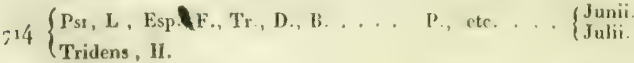

715 Cuspıs, II, Tr........ \{ Bavaria. . . Junii. 


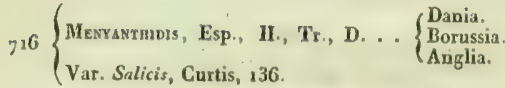

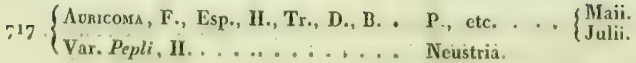

7\$8 Rumcis, L., etc. . . . . . E Europa..... Perannat.

$719\left\{\begin{array}{l}\text { Euphonde, F, W. V., II., Tr., D. Gallia, ete. . Maii. } \\ \text { Var. Cyparissia, H. }\end{array}\right.$

$720\left\{\begin{array}{l}\text { Eupunasi.e, Borkh., Tr., D., B. . . P., cte. . . . } \\ \text { Euphorbix, Esp. }\end{array}\right.$

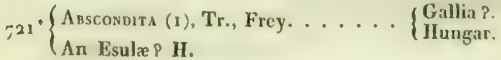

2. GENUS DIPIITERA, Ochs, Tr.

Larvæ arboricolæ, hirsutæ, Bombyciformes. - Antennæ maris crassioves, crenulato-dentatx. Lingua brevis. Thorax rotundatus. $\Lambda \mathrm{b}$ domen coloratum, cristatum. $\Lambda$ læ anticæ concinne depictx, nigro variegatæ.

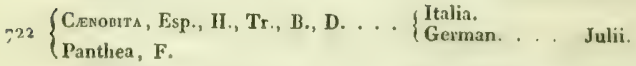

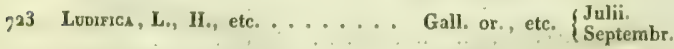

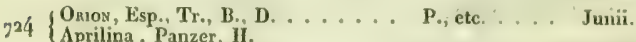

(1) Cl. Treitschle hanc novam opeciem, duabus procedentibus jam nisi Iarvn seceraendis, addit. Insectum perfectom vix ab A. Euphorbia distioclum videtur; sed tarva a Dom. F'reyer depicta valde a larvis Euphorlia el Euphrasioz discrepap. 


\section{GENUS BRYOPIILA, Tr., Boisd. Poecilin, Ochs.}

Larvæ lichenes arborum, murorum saxorumque noctu pascentes, Lithosiformes, tuberculis villosulis, minutis instructa. Puppa in fissuris vel sub lichenibus folliculata. - Lepidoptera minuta. Antennx filiformes. Thorax rotundatus. $\Lambda$ bdomen gracile cristatum. $\Lambda$ læ latiuscula lineis transversis, maculisque ordinariis distinctis.

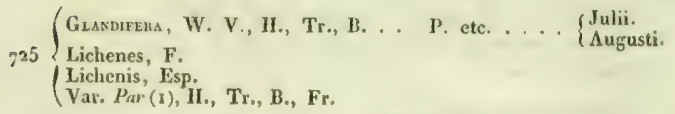

$726\left\{\begin{array}{l}\text { Penus, F., H., Tr., B. . . . . . P., etc. . . A A Musti. } \\ \text { Glandifera, Borkh. }\end{array}\right.$

227 Dakdouini $(2) \ldots \ldots$ Galloprov. . . . Estate.

$728\left\{\begin{array}{l}\text { Velox, H., B: . . . . . . . Monspelii. } \\ \text { Var. Anomala, Ramb., B. }\end{array}\right.$

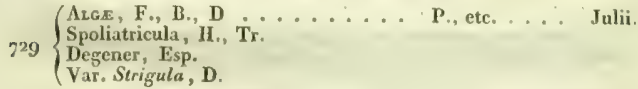

$730^{\circ}$ Mendacura, H. (an var. præcedent. ?).

731 - Calligrapha, H. (an exotica P).

$7^{32}\left\{\begin{array}{l}\text { Ereptricula, Tr., II., Frey, B. . . A Austria. . . Julii. } \\ \text { Var. Troglodyea, Frey }\end{array}\right.$

$733\left\{\begin{array}{l}\text { Receprur.ra , H., Tr., B. . . . . Gallia; ete. Julii. } \\ \text { Strigula, Bork. }\end{array}\right.$

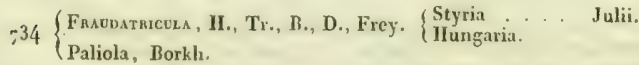

(1) In Gallia meridionali Parisisqque occurrit frequens. Illius larva minime differt * larvis Glandifera genuino.

(ग) Statura $B, Y$ elos; cinereo-plumbea, lineolis undulatis obscurioribus.

In Galloproviacia inventa a Cl. Canteaer, entomologo nobis prograto.- Hec species ac Velos genus forsan efformant proprium. 
BOMBYCOIDES.

735 Lupor, H., Tr., B. (an var, sequentis ?) Gallia. . . . Julii.

${ }_{736}$ Raptricura, H., Tr, B. . . . . . P. etc. : . . Julii. Lupula, D.

$\eta^{3} \eta^{*}$ Ravola, II.

338 . Furvura, $\mathbf{H}$.

Noln. Chalcelonia, II. Tr. e specicbus curopæis delenda, ut civis Americes borealis. 


\section{XVIII. 'TRIBUS AMPIIIPYRIDES, Boisd.}

Larva sxpius elongatie, glaberrima, arboribus lerbisque vescentes. Metamorphosis in folliculo, vel hypogxa.-Antemme in utroque sexu filiformes, rarius in maribus dentata. Lingua sat productit. Palpi disjuncti, ascendentes, solito sub-longiores. Alæ lucidie, anticx sxpius colore tristo, squalido, sub-dccussatx, vix vel obtusissime tectiformes. Dorsum deplanatum. - Volatus nocturnus. - Insecta per diem in locis obscurioribus, latebris, torpentia.

\section{GENUS GonorterA, Lat., Boisd, Dup. Calpe, Tr. Calyptra, Ochs., Steph.}

Larva attennata, clongata, arboricola, capite parvo. Puppa elongata, inter folia folliculata. - Antenna maris sub-pectinata, feminx crenulate. Palpi fronte longiores; articulo tertio clongato. Thorax quadratus, fronte cristato. Dorsum deplanatum. Alx angulatu-dentatx.

$7^{3} 9$ Libatkix, L., etc......... Europ. ... \{ $\begin{aligned} & \text { Junii. } \\ & \text { Septembr. }\end{aligned}$

\section{GENUS SPINTIIEROPS, 13oisd.} dmphipyra, Tr., Boisd. olim.

Larv valde elongatæ, attenuate, Ophiusiformes, subdiales, Legumiyosas pascentes. Puppre folliculatx.-Antenna filiformes. Palpi fronte lonciores. Thorax sotundatus. Dorsmm sub-deplanatum, leve. Alx margine rotundata, maculis ordinariis nitide scriptis.

lloc genus larvis elongatis fere cum Ophinsis congruit, sed insecto perfecto, vix disjungendum ab Amphipyris genuinis.

Z 40 \{ Specthum, F., H., Esp., G., Tr., B. Gall. mer. . Augusti.

74 Cataphanes, H., Tr., G., B..... Gall. mer. . Augusti.

$7 \mathfrak{i}^{2}$ Dievcros, H., Tr., G., B...... Gall. mer. . Julii. 
3. GBNUS AMPIIPYRA, Ochs., Boisd. Amphipyra et Syntomopus, Guenée.

Larva crassiusculx, arboricolx, sub-Sphingiformes, modice elongatx, virides, albo-strigose; segmento penultimo sxpius incrassato, pyratmidali. Puppa sub-folliculata. - Antenna filiformes, elongate. Palpi fronte longiores. Thorax rotundatus. Dorsum deplanatum, lateribus cristulatis. Ala latiores, antice maculis ordinariis obsoletis. sed distinctis. Posticæ rufæe vel rufescentes.

743 Effusa, B., Tr., H. . . . . ...... Gall, mer, ... Julii.

$74\left\{\begin{array}{l}\text { Cinnaromea, Bork, Mllig, Tr., G., B. Gall. alp., etc ' Augusti. } \\ \text { Perfusa, H. }\end{array}\right.$

744 Conica, Esp.

Gen. Syntomopus, Guence.

745 Pyramidea, L., etc. ......... P. etc. .... Julii.

$746\left\{\begin{array}{l}\text { Penrlua, F., II., G., Tr., B.... } \\ \text { Grerman. }\end{array}\right.$

Pyramidina, Esp.

4. GENUS SCOTOPHILA, Hubn.

Pyrophiln, Steph. Philopyra, Gucnée. Amplipyra, Tr., Boisd. olim.

Larva herhicolx, elongatx, virides, Polixformes, albo-lineate. Puppa quisquiliis sepulta, vix folliculata.-Antenna elongat $x$, setiformes. Palpi vix froutem saperantes. Thorax rotundatus. Jorsum deplanitam, læve. Alx anticæ absque maculis ordinariis. Posticæ rufæ vel rufescentes.

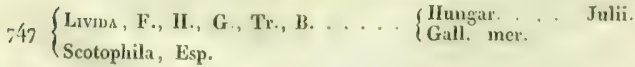

748 Tetra, F., II., Tr., G., B. . . . . \{ Alonspel. . Julii.

749 Tragopogonis, L., cte. ........ P., ete.... Julii. 


\section{GENUS MANIA (1), Tr, Boisd.}

Larva herbivoræ vel arboricolæ, per diem latentes, sub-Noctuxformes, postice sensim incrassata, fusco-marmoratx. Puppa sub-quisquiliis folliculata.-Antennx filiformes. Palpifrontem vix superantes; articulo uitimo breviusculo. Thorax cristulatus. Dorsum maris vix deplanatum, tenue cristulatum, femine carinulatum. Alæ latx, obscuræ ; antica maculis ordinariis nitide scriptis, nervo medio cjusque ramulis pallidis.

$7^{50}$ Mavrs, L., ete. . . . . . . E Europ. .... Julii. ${ }_{751}\left\{\begin{array}{l}\text { Trpica, L., F., G., Tr., B. . . . . Europ. . . . Julii. } \\ \text { Venosa, H. }\end{array}\right.$

6. GENUS RUSINA, Steph., Guénée. Noctua, Boisd. olim. Agrotis, Tr.

I arva obscura, antice attenuata, plantas humiles pascens. Metamorphosis hypogxa. - Antennx maris pectinatx, feminx ciliatx. Palpi fronte evidenter longiores. Alx obscurx, lucidie, litiores maculis or. dinariis obsoletis.

$752\left\{\begin{array}{l}\text { Tesersosa, H., D., Tr., B..... Gallia., etc. Julii. } \\ \text { Ferruginea, Esp. }\end{array}\right.$

(1) Cl. Treitschke in supplemento land, operis (Schmetterlingevon Europa, t. X, p. 2) Noctuam Mauram prope Catocalas refert, quibuscum nullam aftaitatem prabet, nisi twagnitudine insecti perfecti el puppa cesio-pruinosa. Hec species nobis a $T_{y}$ pica secerneada et a Noctuis genuibis procul removenda, baud videtar. 


\title{
XIX. TRIBUS NOCTUIDES.
}

\author{
Noctuelides, Boisd. olim. \\ Noctua, Auct.
}

Larv $x$ obscuræ, glabræ, plantis humilioribus vel radicibus vescentes, per diem latentes. Metamorphosis in terra vel sub quisquiliis.-Ala antica? augustiores, dorso parallele decussatx, haud tectiformes. Dorsum haud cristatum, sæpius deplanatum.-Insecta per diem in herbis occulta, rarissime ad arborum truncos sedentia. - Volatus crepuscularis.

\section{x. GENUS SEGETIA, Stephens (1). Mythimna, Ochs., Tr., Boisd. olim.}

Larvæ cylindricæ, cincreæ, fusco-lineat $x$, graminibus noctu vescentes. Metamorphosis hypogaa.- Antennæ maris dentato-sub-pectinata, feminæ ciliatæ. Palpi mediocres, distantes, fronte breviores; articulo ultimo brevissimo. Thorax rotundatus. Dorsum planiusculum. Alæ antic $x$ obscur $x$, maculis ordinariis nitidis pallidis.

753 Xanthographa, F.,W.V., I.,Tr, D., B. Gall., etc. . Augusti.

254 ImpleXa, H., T., B......... Sicilia

Viscosa, Dahl., Frey.

2. GENUS CERIGO, Stephens.

l'olia, Tr., Boisd. olim. Mythimna, Tr. Suppl.

Larva cylindrica, cincrea, fusco-lineata, plantis humilibus noctu vescens. Metamorphosis hypogat. - Antennx maris dentatx, femina filiformes. Palpi fronte longiores, sub-distantes, compressi, articulo ultimo brevi, cylindrico. Thorax rotundatus. Abdomen leve. Ale la-

(1) Ilocce genus habitu picturaque larvarum ad Leueanias certe accedia, sed insecto perfecto a Noctuis genuinis vix disjungendum. 
tiores, maculis tribus ordinariis nitide scriptis; alis posticis lutcis, margine fusco.

Nota. Primo intuitu, ad hoc genus N. Xnnthochloris et Prospicun attinere videntur, sed habitu larvarum et characteribus insecti perfecti, presertim dorso cristato, cum Hadentdibus melius conveniunt.

Crtherea, F., H., B.

P., etc. .... Julii.

$\{$ Connexa, B

Texta, Esp., Bork., Tr.

\section{GENUS TRIPHENA, Treits., Boisd.}

Larva crass $x$, obscurx, antice sub-attenuata, segmento penultimo maculis cuneatis nigris. Puppx terra sepultx. - Antenna setiformes. Palpi fronte vix longiores. Thorax rotundatus, levis. Dorsum deplitnatum. Ala antic angustiores, elongate, maculis ordinariis distinctis. Alæ posticæ lutex, nigro-fasciatx.

${ }_{7} 56\left\{\begin{array}{l}\text { Llimogusea, F., W. V., H., Tr., G., B. P., etc. . . . Julii. } \\ \text { Agilis, Devill. }\end{array}\right.$

757 Chardinyı, B., D . . . . . R . Russ, mer.

758 Istersecta, II., G., Tर., B....... Gall. occ, etc. Junii.

$759\left\{\begin{array}{l}\text { J Antuins, F., H., Esp, Tr., G., B. . P., etc. . . . Julii. } \\ \text { Fimbria }\end{array}\right.$

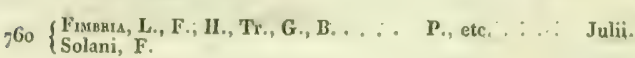
(Orbons, F., G. .......... P., ctc.... Junii. Comes, II., Tr.
Subsequa, Esp.
-61 Pronuba Minor, Devill.
Var. Prosequa, Dahl.
Var, Adsequa, Dahl.
Var, Conuuba, H.

$662\left\{\begin{array}{l}\text { Sursepua, W. V., H., G., Tr., B. . . Europ. hor. . } \\ \text { Var. Consequa, H. }\end{array}\right.$

-63 $\left\{\begin{array}{l}\text { Prosuna, L., etc. . . . . . . . Europ ... } \\ \text { Var. Innuba, Tr., Steph. }\end{array}\right.$ 
4. GENUS OPIGENA, Boisd.

Noctua, Tr., Boisd. olim.

Larva elongata, antice attenuata, Orthosixformis, albo marginata, herbas pascens Metamorphosis subterranea. - Antennæ setiformes. Palpi mediocres, fronte breviores. Thorax antice carinatus, supra bi-cristulatus. Ala antica angustato-elongatx, maculis ordinariis nitide scriptis. Dorsum lave, deplanatum.

764 Pourgoss, F., H., W. V., Tr., G., B. Alpes. . . . Julii. Nigrofulva, Esp.

5. GEINUS CIIERSOTIS, Boisd.

Agrotes et Trachea, Tr. Noctux, Boisd, olim.

Larvx cylindric $x$, elongat $x$, pallid $x$, lineat $x$, plantis humilibus ves centes, fer diem latentes. Metamorphosis hypoga vel sub-folliculati.-Antenna filiformes, maris crassiores, dentatx, rarius pectinata. Palpi vix fronte longiores; articulo ultimo abbreviato. Thorax rotundatus. Dorsum planiusculum. Ala anticx angustiores, maculis ordinariis nitidis.

$+$

$765\left\{\begin{array}{l}\text { Rectangela, F., W. V., Tr. . . . } \\ \text { Var. Anderreggii, B.(minor obscurior). }\end{array}\right.$

$766\left\{\begin{array}{l}\text { Multancula, II., Tr., B. . . . . . Ilelyctia. . . Julii. } \\ \text { Var. Rectangula, B. Iconeg. }\end{array}\right.$ t†

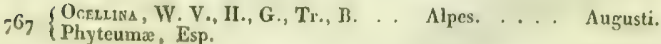

768 Alpestris, B. Icones. . . . . . . Alpes. . . M Maii.

Ht

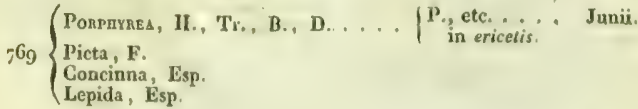


$770\left\{\begin{array}{l}\text { Agarusi (1) . . . . . . . . } \\ \text { Lidia \& B. Index Meth. }\end{array}\right.$

271 Erıce (2). . . . . . . . F \{ Fontainebleau, Junii:

$\mathrm{Ht+t}$

$77^{2}$ PLecta, L., etc......... P., etc. .... Junii.

in3 Leecogasten, Tr, B., Frey. . . . . S Sicilia .... Junii.

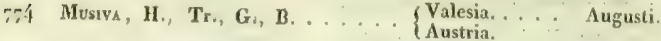

\section{GENUS NOCTUA, Treits.}

Nocture, Boisd, olim.

Larve cylindricx, postice incrassate, obscura, sub-holosericex, maculis in segmento penultimo nigris, cuncatis; plantis humilioribus noctu vescentes, per diem sub quisquiliis latentes. Metamorphosis hypogax. - Antenna maris sub-pectinatxe. Palpi vix fronte longiores, articulo ultimo brevi. Thorax rotundatus. Dorsum sub-deplanatum. Alx sntica latiuscula, maculis ordinariis distinctis.

\section{$+$}

${ }_{77} 5$ ShGattrfen, H., Tr., G., B. . . . V Valesia. . . . Julii.

\{var. A. (obscurior)............ Mustria.

776 Framerta, F., II., Tr., G., B. ... Yalesia. :". Junii. t+

$777\left\{\begin{array}{l}\text { C. Nigrum, L., F } \\ \text { Nun Atrum, Bork }\end{array}\right.$

Gothica var., Esp.

(1) Noctur Agathina Duponrhel qua in ericetis Gallia centralis occurrit et qua dieitur Iidia a multis Kntomologis, valde diflert a figuris Lidia Hubueri et Crameri; quamvis deseriptio $\mathrm{Cl}$. Dom. Treilschke (qui tabulas anctorum amborum affert) nonnilit cum illa specie nostra Gallica congruat. Lidia autem genuioa quo in Gallia australi habitat valde diversa ab ista preudo-Lidia, et ad Agrot, tritici prope accedit.

(2) Afrois certe $N$. Agathine at paulo major; ale antica cinereo.rubicundic, strigis transversis paltidioribus, maculis ordinariis cincreo-albidis, strigula nigrat neparalis, costa plagaque basali linea media divisa, cinereo-albidis; ala postica albido-infuscatue. An. teana maris sub-pectinata. 


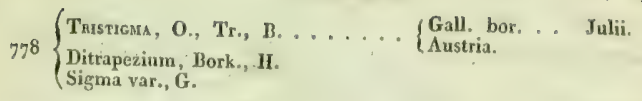

$7 \% 9\left\{\begin{array}{l}\text { Trangulum, O., Tr., B. . . . Gallix, etc. . } \\ \text { Sigma, Esp., II., G. }\end{array}\right.$

$280\left\{\begin{array}{l}\text { Ruonaonea, Esp., Tr., G., B. . . P., etc. ... } \\ \text { Stigmatica, II. }\end{array}\right.$

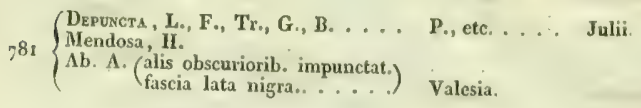

$+t+$

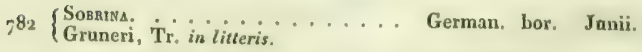

${ }^{283} \cdot \mathrm{F}_{\Delta C E T A}, \mathrm{Tr}_{\mathrm{K}} \ldots \ldots \ldots$ Neapolis.

784 Cosfrus, Tr., B., Di, Fr. . . . Breslau.

$785\left\{\begin{array}{l}\text { Benı, Bork., Tr., G., B. . . . N Neustria, etc. Augusti. } \\ \text { Quadratum, H. } \\ \text { liubi, Vieweg. }\end{array}\right.$

286 Punicea, H., G., Tr., B., Frey... \{ $\begin{aligned} & \text { NNeustria. . . Julii. } \\ & \text { Bayaria. }\end{aligned}$

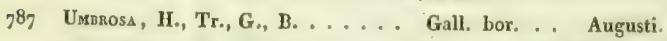

$788\left\{\begin{array}{l}\text { Leucoguspu, H., Tr., B. . . . . G Gall, bor. Septembr. } \\ \text { Var. Lepetitii, B. Icones. }\end{array}\right.$

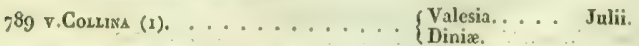

$790\left\{\begin{array}{l}\text { Festivs, W. V., Tr., G., B. . . . Gall, bor., etc. Julii. } \\ \text { Mendica, F. } \\ \text { Primulæ, Esp. } \\ \text { Var. Dahlii, G. }\end{array}\right.$

791 Damu, H. $_{*}$ Tr., B. . . . . . . Austria..... Junii.

$79^{2}\left\{\begin{array}{l}\text { BadmeA, F., W. V.; H., Tr., B. . P., etc..... Junii. } \\ \text { Fragarix, Bork. } \\ \text { Lucifera, Esp. }\end{array}\right.$

(i) Valde afinis Festive. Alæ abtice luteo-corticina, incarnato fuscoque variegata, inacula media nigra stigmatibusqua flavis, postica ciaerea. 
$79^{3}$ Candeuszeva, W. V., II., Tr., G., B. $\left\{\begin{array}{l}\text { Valesia..... Jumi. } \\ \text { Austria }\end{array}\right.$

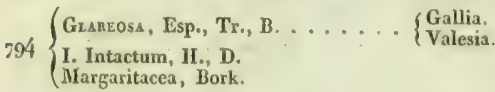

\section{$t+t+t$}

$29^{5}\left\{\begin{array}{l}\text { Bass, F., Bork., H., Tr., G., B. . . P., ete. . . . } \\ \text { Tricomma, Esp. }\end{array}\right.$

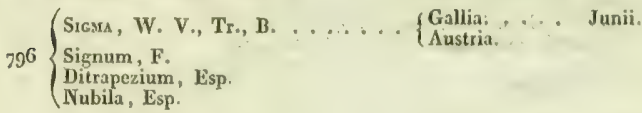

7. GENUS SPALOTIS, Boisd.

Noctua, Boisd, olim. Noctuce, Agrotes et Amphipyra, Tr.

Larre cylindriex, obscura, maculis dorsalibas cuneatis; per diem latentes: plantis humiliorilus noctu vescentes. Metannorphosis hypogæa. Antennæ elongata, filiformes, maris sub-crassiores. Palpi vix fronte longiores; articulo ultimo breviusculo. Thorax rotmulatus. Dorsum deplanatum. Alæ' sub-elongatæ, lucidx, sæpius murinæ maculis ordinariis distinctis.

IIoce genus Noctuas genuinas Agrotibus alarum forma scripturaque conjungit.

$+$

$797\left\{\begin{array}{l}\text { AvGen, F., H., Tr., G., B. . . . G Gall. bor. etc. Julii. } \\ \text { Omega, Esp. } \\ \text { Assimulans, Bork. } \\ \text { Hippophaes, H-G. }\end{array}\right.$

$79^{8}$ Dumetonus, B., H.-G. . . . . . Dinix. . . . . Julii.

tt

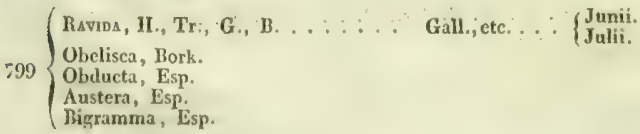


800 Sevrltos, B. . . . . . $\begin{aligned} & \text { Russia. } \\ & \text { Dania. }\end{aligned}$

8o1 $\left\{\begin{array}{l}\text { Senna, II-G., Frey., Tr., B. Icones. . Valesia..... Augusti. } \\ \text { Valdensis, B. Index Meth. }\end{array}\right.$

ttt

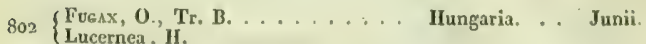

$803\left\{\begin{array}{l}\text { Precox, L., F., Tr., D., B. . . . German. . . . Julii. } \\ \text { Preceps, H. }\end{array}\right.$

tt+t

804 Cataleuca, B., Anderregs. ..... $\begin{aligned} & \text { Ilelvet. . . . Augusti. } \\ & \text { Pyren. } \\ & \text { Iispan. }\end{aligned}$

805v. $\left\{\begin{array}{l}\text { Butuvis, II., Tr. ....... } \\ \text { Var. Monoratina, Donzel. }\end{array}\right.$

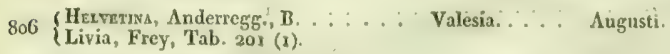

\section{tttt}

So 7 Valesiaca, Anderregg, B. .... $\left\{\begin{array}{l}\text { Russ, mer. } \\ \text { Valesia..... Aprilis. }\end{array}\right.$

808 . Renigers, II., Tr., G. ........ IIungaria. $t+t+t$

809 Conrusa (2) . . . . . . . . R Russ. mer.

8 ro $\left\{\begin{array}{l}\text { Fussmora, II., Esp., G., TR., B. } \\ \text { Maravigna ; D. }\end{array}\right.$

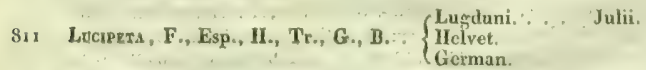

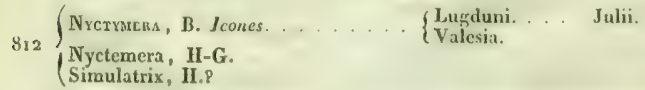

(1) Noctua Livie Freyer, tab. 95. Huc haud atlinet, potias od $A$. Cos referenda.

(2) Statura et aftinitas Fimbriolas : ala antica obscure cinerea, strigis transtersis ma. culisque tribus ordinariis paltidioribus; postica pallidx, margine fusco limbato. Inventa in Russia meridionali a D. Kindermann. 
813 Sininica, B. lcones. : ...... \{ $\begin{aligned} & \text { Ilelvetia. } \\ & \text { Sibiria. }\end{aligned}$

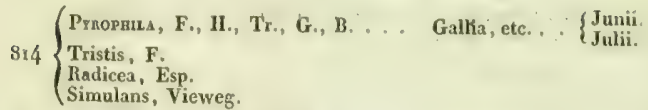

815 Porrcols, B. ........... Cap. nord.

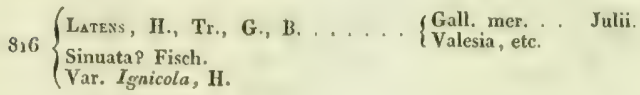

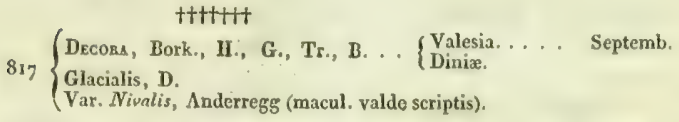

818 v. Gruv, Donzel. . . . . . . . . Dinix. ... . Augusti.

8 rg $^{*}$ Gruszscens, F., Tr., B. . . . . B Breslau.

\section{GENUS AGROTIS, Ochs., Tr.}

Noctur, Boisd. olim.

Larvx squalidx, pigra, livida, sub-vermiformes, subterranex, vel sub lapidibus, muscis, etc., latentes, radices corrodentes, punctis verrucosis, rarius lineis obscuris preditx; segmento primo scutellato; pedibus membranaceis, hrevibus. Metamorphosis hypogaa. - Antennx clongatx, maris dentato-sub-pectinatx, rarius erenatx. Palpi fronte vix longiores. Thorax robustior, rotundatus. Dorsum plus minusve depressum. Alx oblongx, sub-elongatx, maculis tribus ordinariis sæepius distinctissimis.

$820\left\{\begin{array}{l}\text { Agricols, B., H-G. . } \\ \text { Var. Conspicua, H. (1). }\end{array} \cdots\left\{\begin{array}{l}\text { Pyren. } \ldots \ldots \text { Julii. } \\ \text { Ilispan. } \\ \text { Gall.mer. }\end{array}\right.\right.$

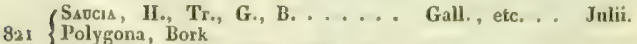
$\left\{\begin{array}{l}\text { Polygona, Bork } \\ \text { Var, Equa, II., G., Tr. }\end{array}\right.$

(1) Jam apud Borkhausen noctua nomine Contricua nuncupatur. 
822 Surrusa, F., II., G., Tri, B. . . . Gall. bor., etc. $\left\{\begin{array}{l}\text { Julii. } \\ \text { Augus }\end{array}\right.$

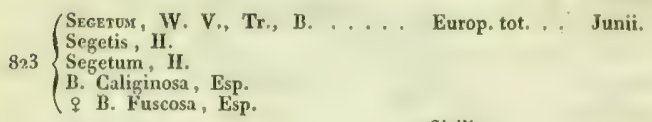

(1) Media inter $\mathrm{Agr}_{\mathrm{G}}$ Cos et Segetum : alis anticis angustioribus cinereo-fuscis, strigis transversis, Jentatis, obscurioribus ; maculis ordinariis albido-cinereis ; rotunda dilutior, oblonga, lougitudinaliter posita; alis posticis albidis.

(2) Stitura Tritici, at ab omnibus facile distincta = ala sordide cineresc, striga subterminali denticulata, maculis ordinariis cavis punctulisqne marginalibus nigris; al $x$ postics albar. - In Russia meridionali inventa a D. Kisilermann. 


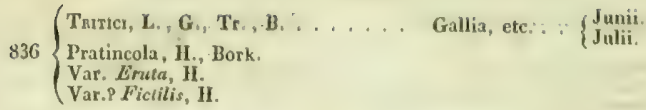

837 Segrizls. . .......... Hungar.

838 Livi, Cram., H., Tr.P. . . . . . Gall, mer.

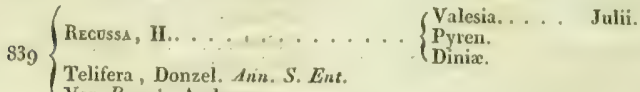

Var. Bromi, Anderregg.

$840\left\{\begin{array}{l}\text { Obelsscs, W. V., H., G., Tr., B. ... Gall. mer., etc. } \\ \text { Molothina, Esp. } \\ \text { Var. Villiersii, Guenée, Ann. S. Ent. }\end{array}\right.$

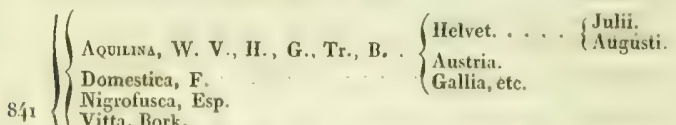

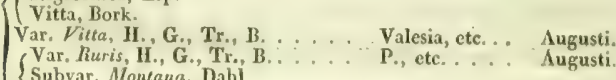
Subvar. Montana, Dahl.
Aberrat. Temera, H. $39^{3}$

842 - TRIFIDA, Fisch. . . . . . . . Russ. mer.

$8\}_{3}$ Signifena, H., Bork, Tr., G., B. . . A Alpes..... Julii

$844\left\{\begin{array}{l}\text { Foncipora, W. V., II, Tr., G., B. : Hungat. } \\ \text { Signifera, F. } \\ \text { Denticulosa, Esp. }\end{array}\right.$

845 v.Fusca, B. Icones, Anderregg. . . . Valesia, . . . Junii.

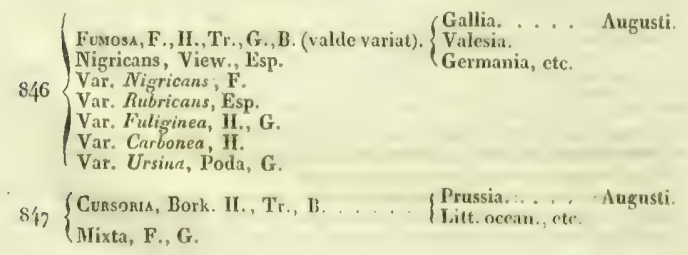


$848\left\{\begin{array}{l}\text { Hup (1), II., Tr., B., Froy. . . . P } \\ \text { Desillii, Pierret, Ann, S, Ent. }\end{array}\right.$

849 Desentorumr (2). . . . . . . . Russ. mer.

$850^{\circ}$ Sagttta, H. . . . . . . . Russ, mer.

851 Signata, B. Icones.......... Alsatia. ..... Julii.

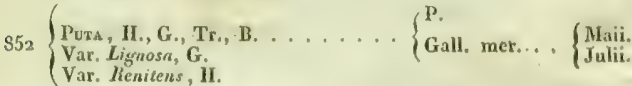
$\$ 53\left\{\begin{array}{l}\text { Purris, L., F., H., Tr. , D., B. . . G Gall, bor. , etc. Junii. } \\ \text { Lignosa, H. }\end{array}\right.$

$854^{\circ}$ Fatrdica, II. . . . . . . Patria?

855 Calligera, F., H., Bork. Tr., G., B... . P., etc. . : … Augusti. $\left\{\begin{array}{l}\text { Clavis, Esp. } \\ \text { Vas. Trigonalis, Esp. }\end{array}\right.$

\$56. Lata, Tr., Frey, Heeger. . . . . Sicilia.

$857\left\{\begin{array}{l}\text { Cusssa, II., Tr., G., B. . . . . . . P., ctc. . . . Julii. } \\ \text { Segetum, Esp. } \\ \text { Var Tritici, }\end{array}\right.$

Nota. Agr. nnnexn, Tr., Boisd., e speciebus europxis delenda, ut hospes Americ borealis.

9. GENUS HELIOPHOBUS, Boisd, Steph., Guenée. Hadena, $\mathrm{Tr}$.

Larvæ vittatæ, crass $x$, sub-obscuræ, fusco lineatæ, per diem latentes, plantas humiliore's pascentes; punctis verrucosis sub-nullis. Metamorphosis hypogxa. - Antenne maris late et ad apicem usque pectinat $x$, femin $x$ setiformes. Palpi ordinarii. Thorax villosus, sub-quadratus, supra rotundatus. Abdomen lave, cylindricum, in feminis

( 1 ) Dom. Bottin Desylles, circa St,-Sauveur-le-Vicomte, hujus rara speciei in sabulosis Oeeani Britannici, puppam reperit el individua concinoe scripta obtinuit.

(2) Sub-aflinis Riparia : alwe antica angusto, cinerex, strigis tribus punctisque marginalibus nigris; maculis ordinariis fuscis ; rotunda longitudinali, minuta ; ala posticu nives; omaibus subtus albis, puncto discoidali nigro. - Inventa in Russia meridionali (3). Kindermann. 
crassius. Alx fuscx, maculis ordinariis, distinctissimis, pallidis, nervis dilucidis.

858 Oness, B., D., Tr. . . . . . . G Galloprov. . . Julii.

85y Optabris, B. Içones.......... Sicilia..... Octobris.

860 Botrica, B., Ramb. Faun. And. ... Hispan. mer.

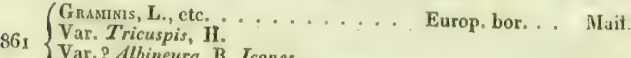
Var.? Albineurn, B. Icones.

Var. Cerapteryx Ifibernicus, Curt.

862 Hinta, H., B.............. Massilia.

Russ, mer.

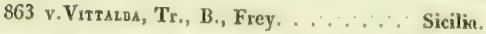

$864\left\{\begin{array}{l}\text { Popolanis, F, Tr., B. . . . . . G Gall, bor, , ete. Julii. } \\ \text { Lolii, Esp., D. } \\ \text { Graminis, }\end{array}\right.$ 


\section{TRIBUS HADENIDES, Noctua, Auct.}

Larva 16-podx, herbas, radices, vel folia arborum pascentes, glabra, colore variabili. Metamorphoses diversa, sxpius subterranex. $-\Lambda 1 x$ insecti perfecti tectiformes. $\Lambda$ bdomen dorso cristatum. - Insecta sxpius ad arborum truncos per diem quiescentia.

1. GENUS LUPERINA, Boisd.

IIadence et Xylino, Tr. Xylophasice, Steph., Guénéc.

Larvæ crassx, squalidx, sub-vermiformes, fusco vittate, sxpius punctis verrucosis plus minusve distinctis proditx, per diem latentes ad radices plantarum, vel subterrancx. Metamorphosis hypogxa. Antennx in utroque sexu filiformes, rarius in maribus crenulatopectinat $x$. Palpi ordinarii. Thorax quadrato-rotuadatus. Alæ autic maculis ordipariis inscriptx.

$$
+ \text { IIrdena, Tr. }
$$

$865\left\{\begin{array}{l}\text { Levcopmes, Bork.,W.V.,H.,Tr.,D.,B. P., etc. . . . Junii. } \\ \text { B. Fulminea, F. Devill, } \\ \text { B. Vestigialis, Esp., Devill. }\end{array}\right.$

it Apamea, $\mathrm{Tr}$.

$866\left\{\begin{array}{l}\text { Cesputıs, W. V., F., II., Tr., D., B. }\left\{\begin{array}{l}\text { Gallia. .... Augusti. } \\ \text { Germania. }\end{array} \text { Hordei, Schrank. }\right.\end{array}\right.$

867 Desuruesı (1). . . . . . . . . Litt. occan. Britan.

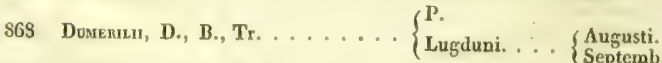

S6g Testacen, W. V., H., Tr., D., B. . . P., etc. .... Septemb.

(1) Statura Dumerilii : ale antice fusce, strigis ordinariis obscurioribus, striga fulgurali albido-cinerea, macula reoiformi albo vitide seripta, macula orbiculari ovata, pallida, iotus infuscata, extus fusco circumdatu, oblique posita. Alos postice albidae, - Fewioam tanturn novi, iode de ablenvis nil dicendum.-Norninata in honoreso D. Bottin. Desylles, bintomolog. oculatissimi, qui bane speciem circa St. Sauvenr-le-Vicomte invenit. 
$+t+$ Apamea et Mamestro, $\mathrm{Tr}$.

$8_{70}$ Roserıı, D. ............ Lugduni.

87x Remandi, B. Ind. M. Suppl. . . . . . Palud, Gall. bor.

$8_{72}\left\{\begin{array}{l}\text { Inresta, O., Tr., D., B....... P. etc. .... } \\ \text { Sordida, Bork. } \\ \text { Anceps, Hunii. } \\ \text { Aliena, D., pl. J02. }\end{array}\right.$

$87^{3}$ Alsicolon, H., Tr., D., B. ...... P. etc, ... Junii.

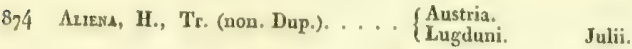

$8_{75}\left\{\begin{array}{l}\text { Asject., II., B. . . . . . . . . . } \\ \text { Nigricans, View. Tr., Fr. }\end{array}\right.$

Var, Fribolus, B. Icones.

$8{ }^{2} 6\left\{\begin{array}{l}\text { Funva, W. V., H., Tr., B...... } \\ \text { Freyeri B gié., Frey. }\end{array}\right.$

t+tt Xylina, Tr. Suppl.

877 Viness, L., etc. . . . . . . . . Gallia, etc. . Julii.

tttt+ Xylina, Tr.

8,8 Zollikorfer, Frey......... Hungaria.

8,9 $\left\{\begin{array}{l}\text { Laterita, Esp., Tr. D., B., Fr. . . } \\ \text { Molochina, H. }\end{array}\right.$

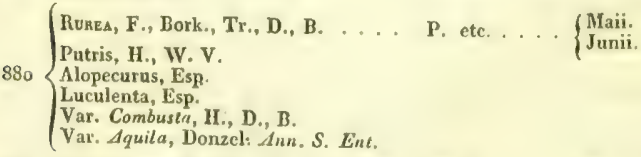

881 Scolopacim, H., Esp., Tr., D., B., Fr. P. etc. . . . Augusti.

882 v. Leinen, Frey, B. Icones. . . . . . IIngaria.

883 Pinastru, L., etc. . . . . . . . P. etc. ... Maii. $884\left\{\begin{array}{l}\text { IIeparica, W. V., Bork., Tr., D., B. Gall, occid. . Junii. } \\ \text { Characterea, H. }\end{array}\right.$

ar. Implex, II. $\eta^{3} 9$. 
$885\left\{\begin{array}{l}\text { Lrrooxusss, W. V., F., H., Tr. . . . P. etc. . . . Julii. } \\ \text { Sublustris, Esp. } \\ \text { Var. Mlusicalis, Esp. }\end{array}\right.$

(Var. Musicalis, Esp.

$886\left\{\begin{array}{l}\text { Ponyodon, L., etc. } \\ \text { Radicea, H., F. } \\ \text { Occulta, Esp. }\end{array}\right.$

P. etc. . . J Julii.

tt+tft Xylina, Tr.

887 \{ Conspiclulakis, L.,Esp., H., Tr., D., B.

P....... Aprilis.

\section{htthtt}

888 Tespru, Thunb., H., Tr., G., B.... $\begin{aligned} & \text { Valesia. } \\ & \text { Suecia. .... Julii. }\end{aligned}$

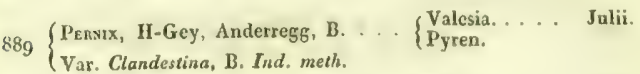

890 Mallzand, B., H-Gey, Anderregg. . Valesía.... Julii.

$8_{9 s}$ - Bugross, B., Anderregg (1)..... Valesia. Julii.

tttt+tt Apamea, Tr.

$89^{2}$ Basılusea, F., Esp., II., Tr., D., B. . P. etc. .... Maìi.

(Genıs, Tr., B., Fr. (valde pariat.): . P. etc. . . . Maii.

Gemina, ơ. II. 482 .

Satura, Bork.

$8_{9} 3\{$ Var. Remiss $n$, H., Fr.

Var. Anceps, $\mathbf{D}$

Var. Submissa, Dahl.

894 Unanints, II., Tr......... \{ $\begin{aligned} & \text { Gallia. . . . Junii. } \\ & \text { German. }\end{aligned}$

(Diprms, Bork., Esp., Tr, II., D. ... P. etc. ..... Junii. Oculea, F.

$8,5\left\{\begin{array}{l}\text { Var. Nictitans, } 11 . \\ \text { Var. Secalina, } 1 \text {. }\end{array}\right.$

Var. Secalina, H.
Var. Leucostigma, Esp.

Var. \& Vilis, 11. 5is.

\$g6 Opmograxna, Esp., H., Tr., D., B. . $\left\{\begin{array}{l}\text { Gall. bor.... Junii. } \\ \text { Hungar. }\end{array}\right.$

$897\left\{\begin{array}{l}\text { Ledcostigars, H., Tr., D., B. . . . . Gall. bor. . J Julii. } \\ \text { Var. Fibrosa, H. }\end{array}\right.$

(1) Mihi iavisa. Mat, Dom. Byringer. 
t+tttitt An Gen, proprium ?

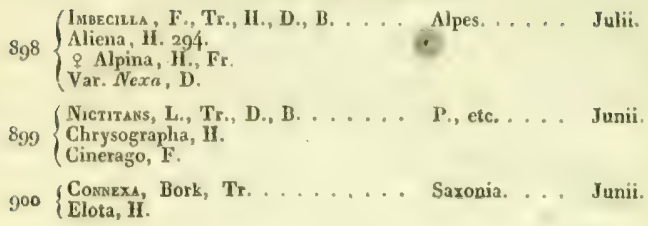

\section{GENUS APAMEA.}

Apamece, 'T1. Miani, Steph.

Larva minutx, vermiformes, decoloratx, pallide lineat $x$, inter vaginas graminum, radices prope degentes. Puppa quisquiliis vel terra sepultax. - Insecti exigua, ad arborum truncos sedentia. - Antemnir filiformes, maris crassiores. Palpi breviores. Thorax rotundatus. Dorsum valde cristulatum. Alx minutx, fusc $x$ snaculis ordinariis nitidis.

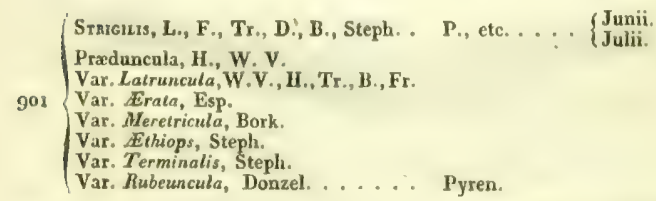

902 v.Surfuauncuna, Tr., Frey. . . . . . Hungaria. . . Angusti.

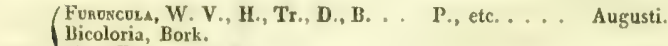
903 Var. Erratricula, H.
Var, Pulmonaria, Dup.
(Var. Viscluncula, H. 96 .

э04 Capriuncula, Tr., D., B. . . . . . . Alpes. ..... Augusti.

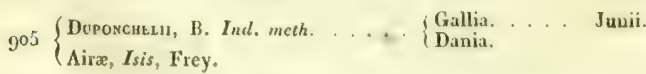

906 v.Microglossa, Ramb., Faun. And. . Hispan. mer. 
907 - Eramatcora, Frey.(an var, Furunculae?) German.

908 Sigralis, Tr. . . . . . . . . . Hungar. .... Julii.

Noln: Apamea Haworthii, Curtis, 260, non Apamen genuina, ut verisirmile exotica.

3. GENUS BRITHYA, Habner.

Glottula, Guen. Cocytia, Tr, Madcua, B., Faun. Madag,

Larpx glabræ, transversim nigro fasciatæ, in foliis, bulbis et ad vaginas pancratiorum degentes. Metamorphosis hypogra. - Anterinæ breves filiformes. Palpi breves, graciliores. Lingua brevissima. Thorax convexits, villosus. Abdomen crassius, in utroque sexu cristatuin. Alw obtusæ, obsolete scriptæ.

Nota : Ad hoc Genus attinet $\boldsymbol{B}$. Timais, Cram, America.

go9 $\left\{\begin{array}{l}\text { Pancratu, Cyrill } \\ \text { Dominica, Cram. }\end{array}\right.$

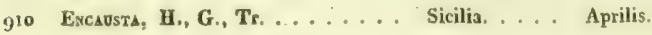

\section{GETUS HADENA.}

Hadenre et Mamostre, Tr., Boisd, olim.

Larvaglabrx, plantis herbaceis foliisque arturum vescentes. Metamorphosis sub terra vel sub quisquiliis. - Antennæ maris simplices, rarius pectinatæ. Palpi frontem haud superantes. Thorax quadratorotundatus. Dorsum maris cristatum. Alx anticx nitide scriptx, maculis ordinariis distinctis, linea terminali in $\geq$ fracto-dentata.

$$
\text { + Agrotis, 'Tr. }
$$

98 Lotouents, W. V., H., Tr., G., B. . P., ctc. . . . Septemb.

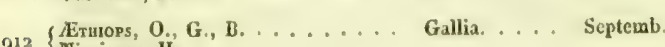

912 Nigricass, II. 
Ht Mamestrn et Misclia, Tr.

913 Persicarle, L., etc. . . . . . . Gall. bor, etc. $\left\{\begin{array}{l}\text { Maii. } \\ \text { Junii }\end{array}\right.$

914 Serpentina, Tr., B. . . . . . Dalmatia.

t† Mamestra, Tr.

$9^{15}$ Brusstce, L., etc. . . . . . . E Erop. . . . Maii.

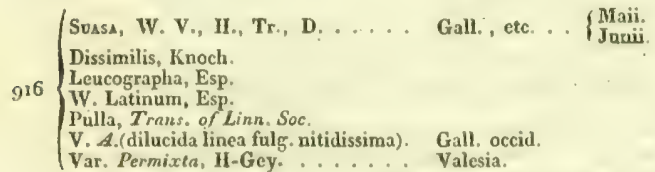

917 Orsaces, L., etc. . . . . . . P. . ctc. . . . $\begin{aligned} & \text { Maii. } \\ & \text { Augusti. }\end{aligned}$

$9_{13}^{13}$ Pisi, L., etc. . . . . . . . . Gall. bor, ctc. Maii.

919* Splendens, H., Tr., B. . . . . . A A Astria.

$t+t+$

$9^{20}$ Ausraus, B., Fr., H-Gey. . . . . G Gall. mer. . A Augusti.

tt+t Xylina, Tr.

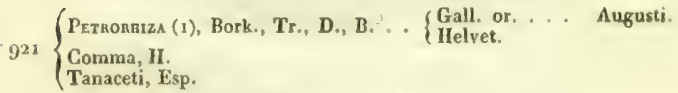

H+t+t Hadena et Mamestra, Tr.

Penegrise, Tr., Fr. 10r....... Litt. Medit... Maii.

Contribulis, B., 0 .

922 Salsola, llamb.

Trimenda, H-Gey.

Var. Blenna, H.

$9^{23}$ Sods, B., D., Tr., H.-Gey. ..... Litt. Medit. . Maii.

92/ Cuenopodu, F., H., Tr., D., B.... P.. etc..... M Magusti.

(1) Signaturis alafum picturaque larvarum ad $N$. Sagittiferent propius accedit, sed babitu, alis tectiformibus ceterisque characteribus cum Hadenis melius conveait. 
II ADENIDES.

ttitt+t Larve nigro flaroque vittatx.

$925^{*}$ Pugsax, H., . . . . . . . . Russ. mer.

$9^{26}$ Treitscriku, B., D., Tr., H-Gey. . Galloprov. . . $\begin{aligned} & \text { Maii. } \\ & \text { Augusti. }\end{aligned}$

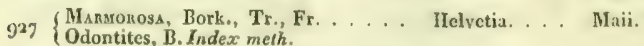

\section{t+tt+ttt Hadena, $\mathbf{T}$.}

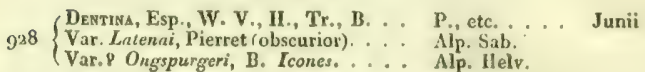

929 Proxisu, H., Tr., D., B., Fr. . . . Alpes...... Junii.

930 Lapro, Dalm., D., B. (an var, sequent.8) Lapponia... . Julii.

$9^{31}\left\{\begin{array}{l}\text { Gladec , II. , Tr., D., B., Fr. .... Alpes.... } \\ \text { Aperta, H-Gey. }\end{array}\left\{\begin{array}{l}\text { Junii. } \\ \text { Julii. }\end{array}\right.\right.$

t+tt+ttt Madena, Tr.

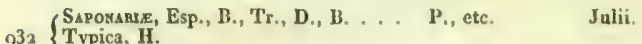

$9^{32}\left\{\begin{array}{l}\text { Typica, H. } \\ \text { Calcatrippx, View. }\end{array}\right.$

933v. $\left\{\begin{array}{l}\text { Grammiprsa, Ramb., Faun. And. . } \\ \text { Cancellata, Kindermann. }\end{array}\right.$

\section{thtthtt}

$9^{34}$ Somserr, B............ Reg. pol. ... Julii.

$9^{35}$ Groenzandica, B........... Finland. ... Julii.

$9^{36}$, Bosra, B. ........... Reg. pol.

$9^{3} \eta$ v.Grusa, Lefebv. Ann. S. Ent. ... Id.

938 v. Exous, Lefebv. . . . . . . . Id. Id.

$9^{3} 9$ v.Leocodon, Eversm. . . . . . . . Russ. orient.

940 Arupucts, L., etc. ......... Europ. .... Junii.

tt+ttt+tt Hadena, Ts.

gí Arica, Tr., B......... \{ $\begin{aligned} & \text { Finland. ... Junii. } \\ & \text { Reg. pol. }\end{aligned}$ 
94a Robnazna, Tr. Frcy, H-Gey.

Var. Feisthamelii, B. Ann., Soc, Ent.

Helvet. .... Angasti.

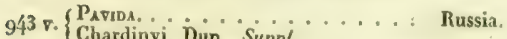

$944\left\{\begin{array}{l}\text { Sarora, W. V., II., Tr., D., B. . . } \\ \text { Porphyrea, Bork., Esp. }\end{array}\right.$

$9\left\{5\left\{\begin{array}{l}\text { Advats, Esp., Tr., D., B. ..... } \\ \text { Valida, H. }\end{array}\right.\right.$

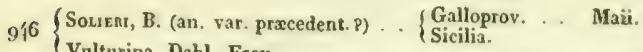

Valturina, Dahl, Frey.

947 Anctica, B. Icones. ......... Sibiria.

948 Rectuses, Esp., H,, Ts., D., B. . Bavaria..... Julii.

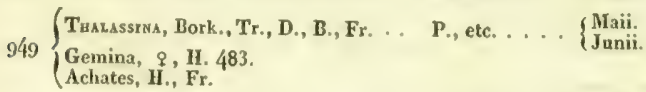

950 Gankdr, B. Icones. . . . . . . . Reg. pol.

951 $\left\{\begin{array}{l}\text { Geniste, Bork., H., Tr, D., B. . . . P., etc. . . . Maii. } \\ \text { W. Latinum, Esp. }\end{array}\right.$

$9^{52}\left\{\begin{array}{l}\text { Contrcus, F., H., Tr., D., B. . . . P., etc. .... } \\ \text { Ariæ, Esp. } \\ \text { Spartii, Bork. }\end{array}\right.$

953 A Apigers, B. Icones (1). . . . . Alp. Delph. . . Junii.

tH+t+tt+t Larva quercicola.

$954\left\{\begin{array}{l}\text { Envgrses, H., Tr., D., B...... } \\ \text { Var. Chioleuca, Dahl. }\end{array}\right.$ 955 v.Miolcuea, Tr., II. . . . . . Sicilia.

956 Convergens, F., 11., Tr., D., 13. . . Gallia, etc. . Augusti.

$9^{5} 7\left\{\begin{array}{l}\text { Distans, H., Tr., D., B., Fr. . . . Gall, mer. . A Augusti. } \\ \text { Var. Suberis, B., D. }\end{array}\right.$

(1) D. Mippert, ia Alpibus ciesa la Grande Chartreuse semel obvia. 
'958, S九ponts, B., D., II-Grey. ..... Galloproy. . Novembr.

959 Protea, Esp., W. V., H., Tr., D., B. Eulop. cent. . Octobris.

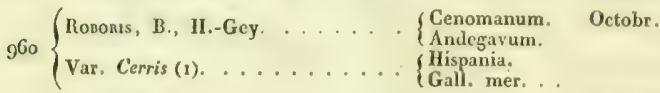

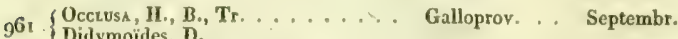

$9^{\mathrm{G}_{2}}$ Foves, Tr., B., H., Frey...... Hungar. ... Septembr.

\section{GENUS PHLOGOPHORA, Tr., Boisd.}

Larvæ glabrx, clongatx, modo virides, modo obscurx, lateraliter oblique fasciatx punctataque, plantis herbaceis noctu vescentes. Metamorphosis sub-terranea. - Antennæ longiores, maris sub-dentatx. Palpi mediocres, villosi; articulo ultimo minuto, tuberculiformi. Lingua producta. Thorax quadrato-rotundatus, antice carinatus. Dorsum maris cristatum, apice fortius barbatum. Alx marmoratx, medio triangulo obscuriori flammiformi signate.

\section{$+$}

g63 Lecrpara, L., H., etc. ........ Gall., etc. . S Maii. $9_{94}$ (Empyaes, II., Tr., D., B. . . . . $\begin{aligned} & \text { Gall. occid. . Junii. } \\ & \text { Etruria. }\end{aligned}$

Flammea, Esp.

965 Scris, H., Tr., D., B. . . . . . $\begin{aligned} & \text { Alp. Delph. . } \\ & \text { German. }\end{aligned}\left\{\begin{array}{l}\text { Junii. } \\ \text { Julii. }\end{array}\right.$

\section{tt}

966 Mericuosı, L., ete........ Europ. .... Y Yutumn.

(1) Hec varietas valde differt ab individuis Galliw centralis, colore cinereo, hepatico sub-variegato, nullo modo viridi tincto. Cieteris characteribas staturaque cum specie typica exacte convenit, - A b. Hambur delecta. 


\section{GEIUS EURHIPIA, Boisd., Guenée. Phlogophora, Tr.}

Larva subdiales, arboricola, glabrx, postice attenuatx. Metamorphosis lypogra. - Antennix filiformes. Palpi frontern superantes; articulo ultimo gracili, elongato. Lingua mediocris. Thorax rotundatus. Dorsum in utroque sexu cristatum. Ano maris bi-penicillato. Alw anticx concinne marmoratx, per quietem plicate.

$9^{6} 7$ Adouataix, H., Tr., D., B., Frey... Gallia mer. . $\begin{aligned} & \text { Maii. } \\ & \text { Septembr. }\end{aligned}$ 968 Buandutmix, B. Icones. . . . . Iispan.

7. GENUS APLECTA, Guenée.

Polia, Tr., Boisd. olim.

Larva glabrx, clongatx, obscuriores, fasciis obliquis sxpius rhombos formantibus, dorso signatx, plantis herbaceis noctu vescentes. Metamorphosis subterranea. - Antenna simplices. Palpi ordinarii. Thorax robustior, antice sinuato-carinatus. Dorsum in utroque sexn cristatum, lateraliter sub-fusciculatum. Alx elongatx, maculis ordinariis nitidis.

\section{$+$}

969 Chxopodipnaca, B., H-Gey., Fr. . $\left\{\begin{array}{l}\text { Litt. maris. } \\ \text { Galloprov. . Junii. }\end{array}\right.$

$970\left\{\begin{array}{l}\text { Serratrukra, O., Tr., D., B. ... } \\ \text { Polyodon, II. }\end{array}\right.$

$9)^{1}$ v.Zrrs (1), Tr., B. . . . . . . Etruria. . . Julii.

$97^{\circ}$ Nonnosi, H. Verz....... Austria. Julii.

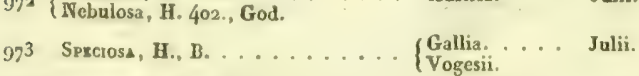

974 Advena, F., H., Tr., D. . . . . . P., etc. $\therefore$. Junii.

(1) Colore, statura primo intuitu Scrratilinec similis, at facile distiocta macula reniformi signo $\zeta$ albo notats, fimbriisque latioribus, cincreo albidoque wariib. 


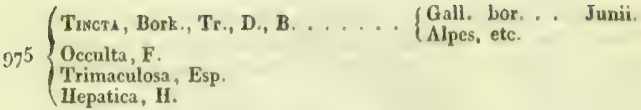
IVedulosa, Naturf., Tr. . . . . . P., etc. . . . Junii.
976 Plebcja, H., D.
Bimaculosa, Esp.
Thapsi, Brahm, Bork.
Polyodon, Illig., F.

977 Occurts, Ross., H., Tr., D., B. . . A Austria. . . Junii.

978 v.Implecta, Lefebv., Aun. S. Ent. . Reg. pol.

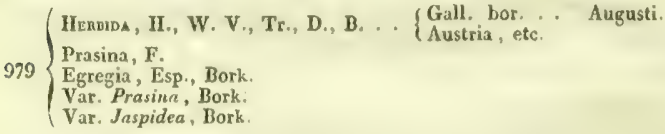

8. GEIVUS AGRIOPIS, Boisd.

Chariptera, Guenée; Misclia, Tr., Steph.

Larva obscura, depressa, arboricola, punctis maculisque rhomboidcis dorso signat3, per diem in fissuris corticum latens. Metamorphosis subterranea, - - ntennæ crassiusculx, sub-crenulatx. Palpi frontem adxquantes; articulo ultimo gracili. Thorax lanatus, quadratus. Dorsum in utroque sexu cristatum. $\Lambda$ læ robustiores, nigro viridique concinne variegata, maculis ordinariis nitide scriptis.

Apruina, L., Esp., Tr., D. . . . E. Europ. . . . Octobr.

Runica, il., W. V., F.

9. GENUS MISELIA, Tr.

Valcria, Steph. Miselia et Chariptera, Guenće.

1.arva arhoricolix, catocaliformes, subtus depresse, nigro maculater, supra cinerex, fusco variegatx, postice carunculis conicis proditx, pedibus membranaceis longioribus. Puppa folliculata. - Antenna 
crassiores, maris dentato-sub-pectinatx, femina sub-filiformes. Palpi ordinarii, hirsuti. Thorax quadratus. Dorsum in utroque sexu cristatum. $\Lambda \mathbf{l} x$ anticx sub-dentatx, maculis ordinariis majoribus, nitide seriptis.

+ Valeria, Germar, Steph., Guen.

$9^{81}$ Oleacias, F., W. V., H., Tr., D., B. $\left\{\begin{array}{l}\text { Gall. or. . . Vere. } \\ \text { Germania. }\end{array}\right.$ $+t$

$9^{82}$ v. Orarculosa (3), Esp., Tr., B.... Ilungar.

t†† Miselia, Steph, Guen.

983 Oxyacartaz, L., etc. . . . . . E. Europ. .... Septembr. 984 \{limaculosa, L., H., Tr. D., B., Curt. Gall., ctc. . Augusti. t+t+ Chariptera, Guen.

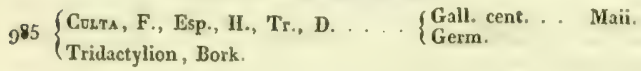

$9^{86}$ Gexaes, Tr., B. . . . . . . . \{ $\begin{aligned} & \text { Valesia..... Septembr. } \\ & \text { Austria. }\end{aligned}$

10. GENUS DIANTHOECIA, Boisd., hev. de Silberm., Guence. Polia, Hadena et Miselia, Tr.

Larva utrinque attenuat $x$, pallide fusce, vel cincrex, sordide oblique fasciatx vel lineatx, in capsulis vel calycibus Caryophyllearum (saltem juveniles) occulte degentes. Puppx subterranex, pectore singulariter prominentes. - Antennæ sub-simplices. Palpi ordinarii, articulo ultimo minuto, taberculiformi. Thorax sub-rotundato-quadratas, antice sinuatus. Dorsam maris cristatum. Oviductus feminx exsertus, valde prominens. Alx breviores, maculis ordinariis distiuctis.

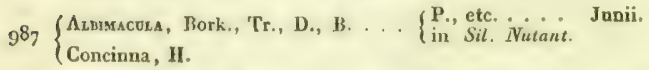

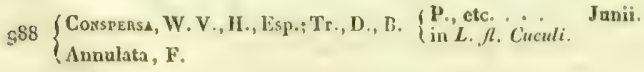

(1) Hæc species sat a duabus sequentibus discrepat, antennis longioribus, alis anticis aigro fuscia, macula reniformi nagaa, retundala, albida, alisque posticis albis nigro limbatis fere ut apud Alchymistam. 
ysy Cosrs, F., Bork., Esp., II., 'Tr., D., B. , H’.. etc. . . . J Junii. $99^{\circ}$ Macmour, B. ........... Gall, mer. . Junii.

$99^{1}$ Xasmocranza, H., Tr. In litteris. . I Ifelvetia. . . Junii.

$99^{2}$ Furcrums, Esp., Tr., II., D., B. . Gall., etc... Junii. $99^{2}$ Polymita, F., If.

$99^{3}$ C.ssrs, W. V., H., Tr., D., B. . . Alpes. ..... Julii.

99 Sulenes, H., B., Tr. ......... Gall. mer. . Juni. $99^{5}$ ч. Duктur, I., G. . . . . . . . . Russ. mer.

$99^{\circ}$ Predrta, $11 . \ldots . . . . . .$. Russ. mer.

997 Capsincou, Esp., Bork., H., Tr., D., B. P., ctc. . . . \{ $\begin{aligned} & \text { Junii. } \\ & \text { Septembr. }\end{aligned}$ $99^{8}\left\{\begin{array}{l}\text { Coconsu, W. W., H., Tr., D., B. . . P., etc. . . . Junii. } \\ \text { Rivularis, F. }\end{array}\right.$

999 Tephrolevca, B. Ann. S. Ent. . . . Alp. Sabaud. . Julii.

ג00o Consica, Ramb. Ann. S. Ent. . . . Corsica.

$100 x\{$ CaApothaga, Bork., D., B. ..... P., etc. ... Junii.

1001 Perplexa, H., Tr., Fr.

1002 Capsopuna, Anderregg. ....... Valesia..... Junii.

${ }_{1003}\left\{\right.$ Ecmu, Bork., H., Tr., D., B. ... \{ $\begin{array}{l}\text { German. . . Augusti. } \\ \text { in Gypsophilo. }\end{array}$ Syngenesix, Scrib.

Brecciaformis, Esp.

\section{Genus ILARUS, Boisd., Guenée.} Eremobia,"Steph. Xanthia, Tr.

Larva $\mathbf{r a l d e}$ elongata, gracilis, postice attenuata, capite crassiusculo, punctis nigris, seriatis, piligeris; semina cerealium manducans. Puppa sub terra tenue folliculata. - Antennx filiformes. Palpi ordinarii, articulo ultimo brevissimo. Thorax villosus, rotundatus. Dorsum in utroque sexu cristatum. Al $x$ marmoratx maculis confusis.

1004 Ochnolzucs, W. V., II., 'I'r,, D., B. Gall. cent. . Julii. 


\section{GENUS POLIA. \\ Police, Tr.}

Larvx glabre, elongatx, subdiales, lineatx. Metamorphosis hypogxa. -Antenua crassiores, sub-dentatx, rarius maris pectinata. Palpi ordinarii, articulo ultimo brevi. Thorax villosus, rotundatus. Dorsum maris cristatum. Alie nebulos $x$, sxpius cinerex vel canescentes, in plerisque flavo-pulverulentæ, linea terminali obsoleta.

+ Antennx simplices.

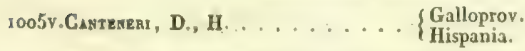

$1006\left\{\begin{array}{l}\text { Drsodes, W. V., H., Tr., D., B. . P., etc. .... Julii. } \\ \text { Clirysozona, Bork, } \\ \text { Flavicincta minor, Esp. }\end{array}\right.$

zoopv.Lotrocixcts, Ramb. Ann. S. Ent. . . Monspelii.

1008 Serena, F., Bork., H., Tr., D, B. . P., ctc. ... $\quad \begin{aligned} & \text { Maii. } \\ & \text { Augusti. }\end{aligned}$

†† Antennze crassiores.

soog Mowtscous, D. . . . . . . . Gall. mer. . Julii.

1010 Cappa, H., Tr., D. . . . . . . Gall, mer., etc. $\left\{\begin{array}{l}\text { Martis. } \\ \text { Junii. }\end{array}\right.$

sorx Cur, L., ctc. ........... P, etc. . , Juhi.

"Septembr.

$10 \times 2$ Cakescens, B., Tr., D. . . . . . . Gall. cent. Octobris.

$\operatorname{sos} 3\left\{\begin{array}{l}\text { Sud , H-Gey., B. . . . . . . . Valesia.... Augusti. } \\ \text { Galii, Anderregg. }\end{array}\right.$

(PonucosA, Ir., Tr., D. Suppl.... \{ $\begin{aligned} & \text { Sardinia. . Augasti. } \\ & \text { Corsica. }\end{aligned}$

$10 \times 4\left\{\begin{array}{l}\text { Asphodeli, Ramb. Ann.S. Ent., H-Gey., D. Suppl. } \\ \text { Senilis, Dahl. }\end{array}\right.$

1015 Semis, B. Icones. . . . . . . G Gall. mer. . Augusti.

3016 Senex, II., 13. . . . . . . . Dalmatia... . Septembr 
101; $\left\{\begin{array}{l}\text { Platiks , Tr., B. . . . . ..... } \\ \text { Var, Senescens, B. olim, Anderregg. }\end{array}\right.$

1018 Veroua................. Galloprov. . Augusti.

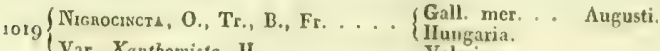

(Var. Xanthomista, H. ........ Valesia.

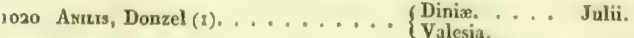

$102 x$ Cerulescens (2).......... Galloprov. .. Septembr.

1022 Ruficincra, H-Gey., B., D. Suppl. . $\left\{\begin{array}{l}\text { Helvet. .... Augusti. } \\ \text { Galloprov. }\end{array}\right.$

(Var. of Dubia, D. Suppl.

Fuviciscta, F., H., Tr., D. . . . P., etc. . . . Augasti.

1023 Flavicincta major, Esp.

(Var. Meridionalis (valde obscurior). . Corsica.

1024 Calvescers, B. (3). . . . . . . Sicilia,

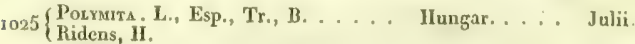

2026v.Durros^, Douzel. Ann. S. Ent. ... Diniæ. .... Julii.

1027 Vreosta, B. Icones. . . . . . . G Gall. mer. . Julii.

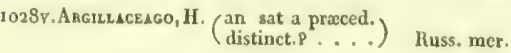

+++ Antennæ pectinatx.

1029 Licuenes, H., Tr., D., B. ... . $\begin{aligned} & \text { Gall. mer. } \\ & \text { Litt. ocean. Brit Juli. }\end{aligned}$

1030 Vındoncta, Dahl., Tr., Fr. .... Sicilia.

I031 " Tephra, H. ............ Dalmat.

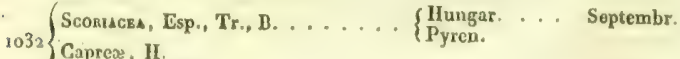

(T'rimacula, Bork.

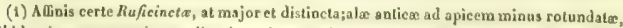
albido cinereoque varix, medio obscuriores, nebulosomobsoleta. Alte postice, infuscatom albida.

(2) Valde aftinis nuficincto at facile distincta punctis marginalibus nigris et delectu atomis rufis in utroque sexu,

(s) Statura P'umicose, at magis Flavicincta adinis. Ala maris cinerascenti-albida, atomis nigricantihus confertim adsperso-nebulesa; alo femina luteo-pulverulenta, ferc ut apud Flavicinctam. Alo postice fusca, lunula media limboque ebscurioribus. 


\section{GENUS POLYPIXEIS.}

Cerigo, Guenée; Mythimna, Tr. Suppl.

Larva clongata, lineata, punctis dorsalibus minutis, plantas humiles pascentes. Metamorphosis hypogxa. - Antennx maris dentatx, femina crenulatx. Palpi distautes, compressi, mediocres. Thorax subrotundato-quadratus. Dorsum cristatum. Ala antica viridi-tinctæ maculis ordinariis daubus nitide scriptis. Alx posticx, fulvæ, nigrulimbatx.

Nota. Hoc genus a Gencre Cerigo facile distinctum, dorso cristato, alisque per quietem tectiformibus, etc.

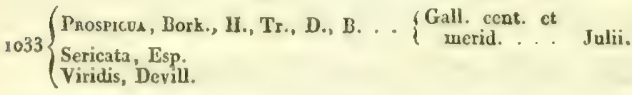

s034 Xantuoculons (1), B. ....... Sicilia.

\section{GEIVUS JASPIDIA}

Plusia, Tr. Polia, Boisd. olim.

Larva et metamorphosis ignotæ. - Antennæ solito longiores, maris crassiores, dentato-crenulatx. Lingua mediocris. Palpi hirsuti, contigui, crassiores; articulo ultimo tuberculiformi. Thorax quadratus, lanatus, antice carinatus, postice penicillatus. Dorsum cristatum. $\Lambda$ læ anticæ virides fascia fusca, maculis ordinariis nullis. - Genus incertæ sedis.

so35 Cessı, L., F., Esp., H., Tr, D., B. $\left\{\begin{array}{l}\text { Suecia. . . . Julii } \\ \text { Styria. }\end{array}\right.$

(1) Prospieua fere duplo major. Als antica latiores, virides, albido adspersa, macula renitormi, nivea, magna, aigrociocia. Ala posticx, fulva, nigro-limbata. Ala omnes subtus cinerea, posticis ad angulum ani fulvescentiluss. 
15. GENUS PIACODES.

Abrostola, Tr. IIadena, Boisd. olim.

Larve elongatx, pallide lincatre, seminibus Umbelliferarum rescentes. Metamorphosis hypogxa. Antennx simplices, maris sub-crassiores. Palpi breves, vix ascendentes, sub-horizontales, articulo ultimo gracili, nudiusculo, inflexo. Thorax rotundatus, debilior, antice sinuatocarinatus, postice bi-penicillatus. Dorsum maris cristatum. Alx lucidx, nitid $x$, sub-fusco-rosex, maculis ordinariis distinctis.

1036 Ansthystina, II., Tr., D., B. . . \{ $\begin{aligned} & \text { Sabaudia } \\ & \text { IIungar. . . Junii. }\end{aligned}$

$1037^{\circ}$ Virgo, Tr. . . . . . . . . Hungar.

1038.v. Spencei (1), B. . . . . . . Italia bor. . Junii.

\section{GENUS ERIOPUS, Tr., Boisd.}

Larve glabra, subdiales, transversim fasciatx, Filices tantum pascentes. Puppe sub terra vel quisquiliis sub-folliculatx. - Antenna simplices. Palpi ultra frontem sub-ascendentes; articulo ultimo mediocri, obtuso. Thorax gracilis, rotundatus, postice bi-penicillatus. Dorsum gracile, in utroque sexu cristatum. Alx latiusculæ, maculis ordinariis distinctis, Pedes antici maris fasciculosi.

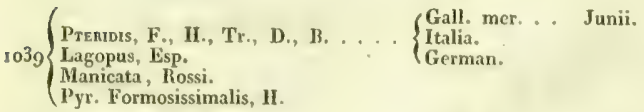

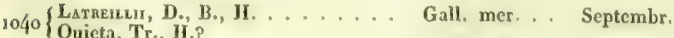

(1) Statura $\mathrm{Pl}$. Consonc, et primo intuitu Amethystine affinis. Alse antica cinereofuscescentes, nitidx, roseo-lota, inaculis costalibus duabus fuscis roseo separatis, strigaque revolata roseo-albida. Alw postic $x$ cinereo-fusca, basi dilutiores.-Hancegregiam speciem reperit in Lombardia Dom. R. Spence. 
17. GENUS THYATYRA, Ochs., Tr., Boisd.

Larva glabre, ferruginex, subdiales, folia Ruborum pascentes; pedibus analibus per quietem resupinatis. Puppx sub-folliculatx. - Antennx maris sub-crenulatæ. Palpi hirsuti; articulo ultimo nudo, longiusculo. Thorax rotundatus, gracilior. Dorsum cristatum. $\Lambda l_{x}$ latiuscula, carneo-marmorato-variegatx, lacidx. Pedes antici vel intermedii maris fasciculosi.

+ Pedes antici fasciculosi.

ro4r Bıtrs, L., etc. . . . . . . . Gall. bor., etc. Junii.

t† Pedes intermedii fasciculosi.

1042 Denass, L., etc. : . . . . . . Gall, bors, etc. Junii 


\section{TRIBUS LEUGANIDES.}

\section{Noctua Auctorum.}

Larv 16-podx, glaberrima, lineatx, plantas humiles pascentes. Me. tamorphosis hypogxa. - Insecta pallida, sxpius straminea, in herbis per diem latentia; dorso levi, nunquam cristato. Alæ tectiformes, lineis transversis maculisque ordinariis sxpius nullis.

\section{GENUS MYTHIMNA.}

Mythimna, Tr., Boisd. olim.

Larva cylindrica, glabra, cinereo-albida, fusco multilineata, graminibus noctu vescens. Metamorphosis subterranca. - Antennæ simplices. Palpi ordinarii, articulo ultimo brevi, tuberculiformi. Thorax rotundatus, villosus. $A l \rightsquigarrow$ latiusculæ, integerrim $x$, maculis ordinariis indicatis. Pedes postici maris dense fasciculosi.

Hocce genus a sequenti tantum differt pedibus maris valde fasciculatis.

$+$

Gallia, etc. . Junii.

æ. GENUS LEUGANIA, Ochs., Tr., Boisd.

Larve cylindricæ, glabræ, per diem latentes, pallidæ, multilineat $x$. Puppa terra sepultæ. - Antennæ simplices. Palpi ordinarii, crassiusculi, articulo ultimo vix conspicuo. Thorax rotundatus, lavis. Alas pallidx, maculis ordinariis nullis, sxpius puncto centrali signatxe

$+$

1044 Conigera, F., W. V., II., Tr., D., B. Gallia, ctc. . Junii

$1045\left\{\begin{array}{l}\text { Albupuncta, F., Illig., H., Tr., D., 13. Gallia, ctc. . J Junii. } \\ \text { Lithargyria, Esp. }\end{array}\right.$ 


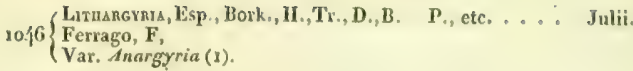

3047 Viт̨luin, H., Tr., D., B. . . . . Gall. mer. . A A gusti.

$2048 \operatorname{Musculos}(2)$, H., Tr., D., B. . . . G Gall. mer. . Julii.

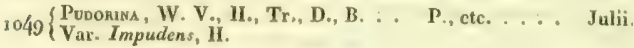

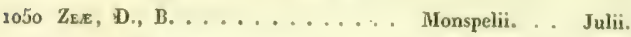

$\operatorname{so51}\left\{\begin{array}{l}\text { Conns, L., Bork, D., B. ...... Gall. bor. .. Junii. } \\ \text { Turbida, H. } \\ \text { Impura, H. }\end{array}\right.$

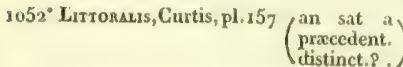

(tistinct.? .) Anglia. .... Julii.

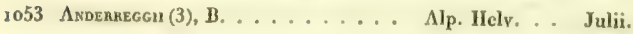

1054 Congaua, H., Tr. ......... Sicilia. .... Junii.

у055 Ripara, Ramb., B........ Gall. mer. . $\left\{\begin{array}{l}\text { Maii. } \\ \text { Septembr. }\end{array}\right.$

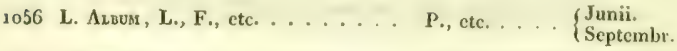

1057 Alopecuru (4). . . . . . . . Taganrok Russix.

$1058\left\{\begin{array}{l}\text { Punctos., Tr., B. . . . . . . . Gall. mer. . Julii. } \\ \text { Boisduvalii, D. } \\ \text { Var.? Putrescens, H. ๆ30. }\end{array}\right.$

(1) Differt a Lithargyria genuina, alis subtus minus mícaceis et defectu penicillo atro ad basin abdominis maris.

(2) Vix hujus Seecris; forsan ad Genus Endagria propius accedens. Metamorphoses desiderantur.

(3) Klegantissima species $L$. Comma sub-affinis at multo minor : ale antica pallide חlavido-fusca venis nigris, superjectis venis niveis; fimbria costaque rubescentibus punctis margioalibus nigris : ala pestica supra nigricantes, subtus albicantes lunula centrali magaa nigra; abdomen apice fulvum.

(4) Minoor L. Impura : alse antico pallide straminea linea media obsoleta, radiis apicalibus funbriaque fuscis; puncto medio albido ad apicem cellulie punctulo nigro adjecto. Ala postica albida. Femina a mare differt defectu linex medio fusca, at amplius saudet punctis nigris, minutis, ultra medium, biseriatis. - Io Russia meridionalia D. Kindermann defecta. 
$1059\left\{\begin{array}{l}\text { Caricrs, Tr. ........ } \begin{array}{l}\text { Sicilia. } \\ \text { Hispania. }\end{array} \\ \text { Albigutta, Dahl. }\end{array}\right.$

1060 Lorexx, D., 1. . . . . . . . . G Gall. mer. . Julii.

logr Obsolera, H., Tr., D., B. ...... Gall., etc. . Septembr.

1062 Anescola, Ramb. ......... Gall, mer. . Julii.

1063 Sicurs, Tr. . . . . . . Sicilia.

ro64. v. Crpera (1), B.............. Calabria,

$065 \operatorname{Scrnpr}(2)$, B. . . . . . . . Monspelii. . Junii.

1066 Dactulidis (3), Ramb., B. . . . . Gall. mer. . Junii.

1067 Montium (4), B., Anderreg5. ..... Sum. Alp.

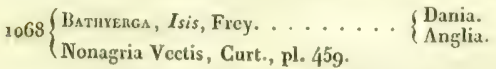

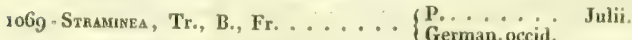

1070 Impona, II., Tr., B........... P, etc. .... Julii.

$\log _{1} \operatorname{Lotos}$, H., B. . . . . . . . . Gall. mer.

$10{ }^{2}$ Elym, ${ }^{\text {Tr}}$, B. . . . . . . . . Prussia,

$\operatorname{rog}_{3}\left\{\begin{array}{l}\text { Pallens, L., F, H., Tr., D., B. . . Europ. .... } \\ \text { Var. Pallida, Bork. } \\ \text { Var.8 Ectypa, H. }\end{array}\right.$

(1) Aftinis et subsimilis $\boldsymbol{L}$. Scirpi : ala anticœ pallide luteo-rufescentes, nervo medio albido, sub-dilatato, puncto medio albido adjecto mioori nigro; also postica albide margine anguste fuscescentes - Mus, D. Escher.

(2) Statura $L$. Zeo, Mla antico paltide cinereo-rufescentes, puucto medio albido strigaque arcuata, obsoleta punctorum obscuriorum; ale postica albida, ad extimunfuscescentes.

(8).Colore Albipuncto vel Lythargyria borealis : statura Zea. Alwa antica pallide fusco-rubricantes, atomis obscurioribus conspersio, puncto medio albido strigaque arcuata, absolela punctorum fuscorum: ala postica nirea.

(6) Aftinis $L$. Scirpi at gracilior et angustior : aloo antica pallide cinereo-rufescentes, atomis mioutissimis obscuris adspersa, puncto melio albido strigaque arcuata, obsoleta, fusea, vix interrupta: alx postica albida, nervis margineque obseurioribus. 
$1074\left\{\begin{array}{l}\text { Punagaridis, II., Tr., D., B. ... } \\ \text { Plragmatidis, Auct. Angl. } \\ \text { Semicana, Esp. }\end{array}\right.$

\section{Genus NONAGria, Tr., Ochs., Boisd.}

Larva elongatx, antice posticeque scutellatx, paludicole, capitc parvo, in caulibus Graminearum vel Cyperaccarum occulte degentes. MCtamorphosis in caulibus. Puppæ capite, tuberculo obliquo, acuto, nasiformi, præditx. - Antenux crassiusculx, maris crenulata. Palpi ultra frontem assurgentes. Thorax lavis, rotundatus. Abdomen elongatum. Alx clongatx, maculis ordinariis sxpius conspicux.

$20-5$ Exraesa, H., Tr., D. . . . . . German. . . Julii.

1076 Junc1 (1), B. . . . . . . . Germ. occid. Julii.

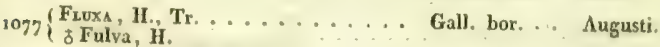

1078 Despecta, Tr., B. ........... Gall. mer. . Julii.

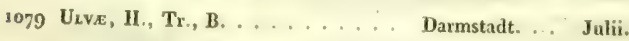

1080 Nevaca, H., Tr., D., B. . . . . D. Darmstadt. . . Augusti.

108 เ $\left\{\begin{array}{l}\text { Ilessn (2), Boist. (an var. Ncurica? ). Darmstalt. . . Augusti. } \\ \text { Neurica, 1I. 659. }\end{array}\right.$

${ }_{2082}\left\{\begin{array}{l}\text { llospes, Tr., Frey, tab. 21. . . . S Sicilia. } \\ \text { Alssoluta, Dahl. }\end{array}\right.$

1083 Nexa, U., Tr., B. (non Dup.), Fr. . Gall. bor, etc. Augusti

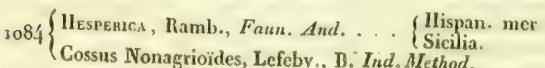

(1) Statura $N$. Estremce illiusque afliois at minor : alao anticae pallide straminex, nerro medio infuseato, maculaque reniformi obsoleta, atbida : ala postico pigricantes.

(2) Ale antica nigro-fuscex, macula reniformi albido, intus fusca : alde postica pallida Dom. Ifess, qui alounde Nonagrias circa Darmstadt educit, mihi ut variet. Ncurice hane speciem misit. Dom. Treilschke quoque in synonymia ad Ncuricam genuidam refet. 
LEUCANIDES.

1085 Paldicora, H., Tr., B. . . . . . Gall. centr. . A A gusti.

Var. Guttans, II. . . . . . . . Darmstadt.

${ }_{1086}\left\{\begin{array}{l}\text { Canswe, Tr., D., B. . . . . . . . Gall. bor., etc. Augusti. } \\ \text { Algre, Esp., Bork. }\end{array}\right.$

1087 Sparganit, Espe, H., Tr., D., B., Fr. P., etc. . . . Augusti.

$1088\left\{\begin{array}{l}\text { Trpue, Esp., Bork., H., Tr., D., B. P., etc. . . . Augusti. } \\ \text { Arundinis, F. }\end{array}\right.$

Yar, Fraterna, Kinderm. ...... Syrmia. 


\section{TRIBUS CARADRINIDES.}

\section{Noctua Auctorum.}

Larv u -podx, minus elongat $x$, plus minusve pilosulx, plantis humilibus vescentes. Metamorphosis sub-folliculata. $-\Lambda \mathrm{l}$ æ plane tectiformes. Dorsum lave, nunquam cristatum. Abdomen breviusculum.

\section{GENUS SIMYRA, Tr. Leucania, Boisd. olim.}

Larvie pilos $x$, sub-Acronyctiformes, plantis herbaceis vescentes, subdiales, tuberculis plus minusve hispidis munita. Puppa folliculatx. - Antennæ crassiuscula, breviuscula, maris dentat $x$. Palpi graciliores. Thorax rotundatus. $\Lambda$ lx antic $x$ pallidx, lanccolat $x$, absque maculis ordinariis.

$1089\left\{\begin{array}{l}\text { Nervoss, F., W. V., H., Tr., D., B. Germania. ... Julii. } \\ \text { Oxyptera, Esp. }\end{array}\right.$

10go.v.Denthosa (1).......... Turcia,

1098 "Dubrosa, Tr. ........... IIungaria.

$\operatorname{sog}^{2}\left\{\begin{array}{l}\text { Venosa, Bork., Tr., D., B. . . . . Gall. oce, etc. Junii. } \\ \text { Degener, H. }\end{array}\right.$

\section{GENUS CARADRINA, Ochs., Tr., Boisd.}

Larve pilosulx, pigrx, antice posticeque attenuat x, depressx, punctis elevatis pilum retrorsum inclinatum gerentibus; noctu plantas lumiles depascentes. Metamorphosis subterranea, sub-folliculata.-Antennæ simplices. Palpi ordinarii. Alx antica integrx, maculis ordinariis sxpius distinctis.

(1) Paulo inajor $S$. Nervosá; ala anticu minus acuta, pallide fusce, costa late, basi marginis interni maculisqur apicalibus strigatis, niveis; ala postica fusca unargine albo. - In Turcia invenia a D, Kindermann. - Mus. D. Rambur. 
$\log _{3}\left(\begin{array}{l}\text { Tnulnes } \\ \text { Quercus, F. }\end{array}\right.$

Trigrammica, Esp.

1094 Bunea, H., Tr., B., Curt. . . . . Gall. bor. . J Junii.

tt

log5 Respersa, W. Y., H., Tr., D., B. . Gall. cent. . Julii.

rog6 Sezini (1), B., Anderregg. ...... Valesia..... Julii.

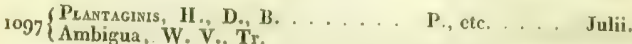

$1098\left\{\begin{array}{l}\text { Buanda, IH., D., B. (an var. sequent. P). P., etc. . . . . Augusti. } \\ \text { Superstes, Ochs., Tr. }\end{array}\right.$

$1099\left\{\begin{array}{l}\text { Taraxacr, HI., D., B. . . . . . . P., etc. . . . Augusti. } \\ \text { Blanda, W. V., Tr. }\end{array}\right.$

1100 Alsiwes, Bork., H., Tr., D., B. . . Gallia. . . . Julii.

1101 Gerkanis , D. Ann. S. Ent. ..... Monspel.

Hispania.

1102 Terkes (2), Kinderm. . . . . . Russ. mer.

${ }_{1103}$ Lesta, Tr., Frey,, II-Gey. . . . . Hungar. . . Julii.

1 104 $\left\{\begin{array}{l}\text { Mlorpueus, View., Tr., B........ Gall, bor. . Junii. } \\ \text { Sepii, H., D. }\end{array}\right.$

$2105{ }^{\circ}$ Gluteosa, Tr. . . . . . . Syrmia.

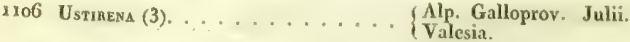

(1) Iacie $6 u b-$ similis Resperse at paulo minor, gracilior : alis anticis arganteo-cinereis, strigis obscurioribus, macula reaiformi strigaque fulgurali rufo-ferrugiaeis : ala postice cinerex, ad basin dilutiores.

(2) Atiois Carad. Morpheus. Ala anticx fusen, strigis obscurioribus undulatis, fascia fulgurali nigra, macula reniforuoi fusco-Intea, extus allido punctulata; postiede albida margine infuscato. - In Russia meridionali inventa a D. Kindermano.

(3) Affinis Kadenit at paulo major et plane distincta. Alo antice eioerex, strigis undalis, transversis, obseurioribus, fascia marginali sub-interrupta nigro-fusca; macula reniformi fusor. Alis postice albidx. 


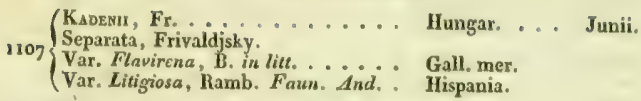

1108 Fuscrcorsis, Ramb. Ann, S. Ent. . Corsica, ... Junii.

Ixog.v.Aspensa, Ramb. . . . . . . . . Gall. mer. . Julii.

$1180^{\circ}$ DasYchí, H. . . . . . . . Patria P

III $\left\{\begin{array}{l}\text { Cudrcularis, W. V., H., B., D. . . . P., etc. . . . . Mugusti. } \\ \text { Quadripuncta, F. }\end{array}\right.$

Segetum, Esp.

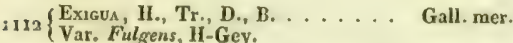

113.v. Prgnce, Ramb. (an aberrat praced.P). Gall, mer.

3. GENUS HYDRILLA, Boisd. Caradrina, Tr.

Larvæ per diem latentes, pubescentes, plantas humiliores pascentes. Metamorphosis hypogxa. - Statura maris Phalceniformis. Femina crassior, pigra. Caput parvum. Antennx filiformes. Palpi subtus villosi, contigui, graciles, vix ascendentes. Thorax villosus, gracilis. Abdomen maris elongatum, gracile, apice penicillatum. Ala latiores, obscurx, tenuiores, maculis ordinariis distinctis.

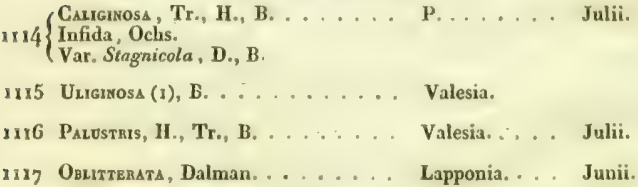

(1) Statura gracilis Breph. Notha. Alx antico cinerex, canescentes, strigis gracilibus serieque punctorum ante apicen nigris, macula reniformi fusca, macula rolunda puncto minutissimo nigro indicata; striga folgurali albida ? ala postico rallide fuscu : ala omaes subtus albiđa, striga communi sub-media, nigra. 


\section{TRIBUS ORTIIOSIDES.}

\section{Noctua Auct.}

Iarve 16-podx, clongatx, glabra, modo plantas humiles, modo folia arborum pascentes. Metamorphosis hypogaa. - Ala insecti perfecti plane tectiformes. Dorsum leve, haud cristatum. - IIxc tribus cum IIadenidilus quamdam affinitatem præbet, sed facile distincta dorso insecti perfecti nunquam cristis predito.

\section{GENUS EPISEMA; Ochs., Tr., Boisd.}

Larva latentes, plantis herbaccis vescentes, lineatx, sxpius oblique fasciatx. Puppe terra sepultx. - Antenna maris late pectinat $x$, femina filiformes. Palpi mediocres. Thorax lanatus, robustior. Alx antica latiores, maculis ordinariis magnis cum macula basali venula junctis.

$+$

$1118^{\circ}$ Odites, H. ........... Russia mer.

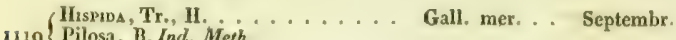
(Hirta, D.

H+

1120.v.Gnuneri, B. Icone.
Sicilia.

IIungar. . . Septembr.

Hungaria.

Gallia.

Valesia

Gallia.

Gall. mer.

Ilungaria. ... Maii. 
2. GENUS ORTHOSIA, Ochs., Tr., Boisd.

Larve undx, subdiales, clongate, modo plantas humiles, modo folit arborum depaseentes, fuscr, vel virides. Metamorphosis subterranea. -Statura robustior. Antenna maris crassiores, plus minusve dentatopectinatæ. Palpi villosi, breviores. Lingua sat producta. Thorax rotundatus, villosus; lineis maculisque ordinaris nitide scriptis.

+ Larvx herbicolx.

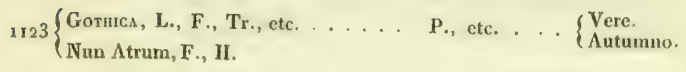$$
12_{24}\left\{\begin{array}{l}
\text { Lirons, L., F., H., etc. . . . . . . Gallia, etc. . S Septembr. } \\
\text { Var. Depuncta, Bork. } \\
\text { Var. Ornatrix, Frey., H. . . . . Italia. }
\end{array}\right.
$$$$
{ }_{1125}\left\{\begin{array}{l}
\text { II ebraica, H., Frey. ......... } \\
\text { I Geminum, D. }
\end{array}\right.
$$$$
3126 \text { Chardaca }(x) \text {. . . . . . . Nuss. mer. }
$$$$
\text { } 1 \times 27 \text { Neglecta, II., Tr., G., B. . . . . Gallia. . . J Julii. }
$$$$
1128 \text { Coecrmacora, F., W. V., II., Tr., D., B. P., etc. . . . Septembr. }
$$$$
1129\left\{\begin{array}{l}
\text { Gnacnus, F., Bork., H., Tr. . . . G Gallia, etc. . Maii. } \\
\text { Collinita, Esp. } \\
\text { Var. Lepida, Bork. } \\
\text { Var. Subplumbea, Curt. }
\end{array}\right.
$$

$1{ }_{130}$ tuevs, H., Tr., D., B. . . . . . . Gallia, cte. . Augusti.

$1131^{\circ}$ Suspecta, II. (an, var. sequent.P). . Patria ?

1132 $\left\{\begin{array}{l}\text { Congenen, H., Tr., 13. . . . . . . P., etc. . . . Augusti. } \\ \text { Var. Caradrina Iners, Tr. }\end{array}\right.$

$1133\left\{\begin{array}{l}\text { Nirida, F. WV. V., H., Tr., D., B. . P., etc. . . . Septembs. } \\ \text { Lucida, Naturf. }\end{array}\right.$ Vaccinii Var., Esp.

(1) Statura Neglecta et sub-aftinis IIebraica : alw anticw cincrex, cupreo sub-nitentes strigis tribus nigris; maculis ordioariis cupreis, triangulis nigris interne coadunatis : als posticx albida, innoculata. 


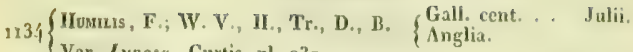

Var. Lunosi, Curtis, pl. 237 .

Pistacina, F., IY. V., II., Tr., B. . . 1', etc. . . Septembr.
Lychnidis, D.

Serina, Esp.

Var, Lychnidis, H., F.

1135 Var. Schanobrena, Esp.

Var. Canarin, Esp.

Var. Lineola, Curtis.

Var. Spharulatina, Curt.

Var, Ferrca, Curt.

Var. Venosa, Curt.

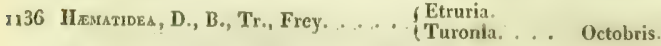

1137 Nennodes, H. . . . . . . . . Dalmatia.

$1138\left\{\begin{array}{l}\text { Rubrucoss, F.,W.V., H., Tr., D., B. .. P., etc. . . . . Apritis. } \\ \text { Mucida, Esp. }\end{array}\right.$

(Var. Mista, 11. 5o9.

1139 Machenta, Tr., D., B., II. 418. . . Gallia, etc... \{ $\begin{aligned} & \text { Augusti. } \\ & \text { Septemb. }\end{aligned}$

t+ Larva arboricola.

$140\left\{\begin{array}{l}\text { Munda, F., W. V., H., Bork., Tr. . Gallia, ctc. . Aprilis. } \\ \text { Var. Bimnculata, Curt. }\end{array}\right.$

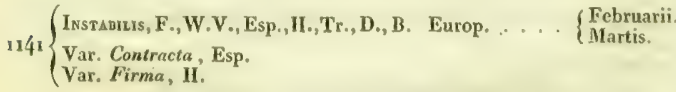

$1142\left\{\begin{array}{l}\text { Ypsmon, W. V., Bork., II., Tr., D., B. Gallia..... Julii. } \\ \text { Corticea, Esp. }\end{array}\right.$

$1 \times 4^{\circ}$ Fankasir, Tr. ............ Hungaria.

$1144\{$ LorA, L., F., II., Tr., D. . . . . P., ctc. . . Septembr,

1145 Opnis, H., Tr., B. . . . . . Austria.... Aprilis.

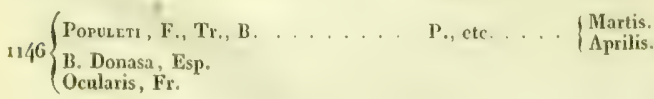


$1147\left\{\begin{array}{l}\text { Srabius, H., Bork., W. V., Tr., D., B. P., etc. . . . } \\ \text { Cerasi \& F. } \\ \text { Var. Juncta, Curt. } \\ \text { Var. Rufannulata, Curt. }\end{array}\right.$

1148 Hrperbore, Dalman. . . . . . Lapponia.

$1149\left\{\begin{array}{l}\text { Carnea, Thunb., Tr., G., B..... Suecia. } \\ \text { Ampla, II. }\end{array}\right.$

(Tecta, H.

\&150 $\left\{\begin{array}{l}\text { MIniosa, F., Bork, W. V., H., D., B. P., etc. . . . } \\ \text { B. Rubricosa, Esp, }\end{array}\right.$

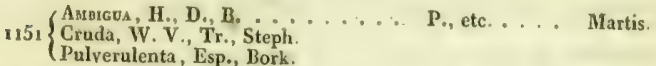

$1152\left\{\begin{array}{l}\text { Rurrcura }, \text { Esp., Tr. ....... } \\ \text { Serpylli, H. } \\ \text { Vall. mer. . Novembr. }\end{array}\right.$

(Var. Ilicis, B. Ind. M. Suppl.

3. GENUS TRACHEA, Ochs., Tr.

Achatea, Steph.

Larva glabra, clongata, viridis, lineata, arboricola. Metamorphosis hypogæa. - Statura robusta. Antennæ filiformes. Lingua brevis. Palpi villosi, brevissimi. Thorax quadrato-rotundatus, densissime villosus. Abdomen abbreviatum, crassiusculum, subtus valde villosum. Alæ robusta; antic $x$ concinne depicta, maculis ordinariis magnis nitide scriptis.

$\times 153\left\{\begin{array}{l}\text { Punipenda, Esp., Tr., D., B, ..... } \\ \text { Flammea, W. V., Ii., F. } \\ \text { B. Spreta, F., Curtis. } \\ \text { V. A. (alis anticis canescenti-cinereis). Lapponia. }\end{array}\right.$ 


\section{GENUS COSMIA, Oclis., Tr.}

Larva virides, albo-strigos $x$, clongatæ, antice attenuatæ, subtus depressæ, capite minuto, inter folia arborum sxpius degentes. Puppx abbreviata, cœsio-pruinosæ, laxe sub-folliculatæ. - Antennæ maris crassiuscula, tenue sub-crenulatæ. Thorax globulosus, lævis. Abdomen conicum. Alæ anticæ area media trigona vel trapeziformi delineatx; maculis ordinariis vix distinctis.

\section{$+$}

$\$ 154$ Drrfusis, L., F., etc. . . . . . . P., etc. . . Julii.

1155 Afruxis, L., F., etc. . . . . . . P., etc. . . Julii.

$1156\left\{\begin{array}{l}\text { Pykaxina, W. V., H., Tr., D., B. . . P., etc. . . . Julii. } \\ \text { Corusca, Esp. }\end{array}\right.$

1857 . Nomrsms, H. . . . . . . . Patria ?

$+t$

1158 Trapezina, L., F., etc. . . . . . P., etc. . . . Julii.|

1159 Abluta, H., Tr., B. . . . . . . Hungaria... . Augusti.

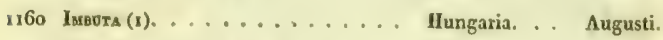

136 I $\begin{aligned} & \text { Forvaco, W. V., H., Tr., D., B. . . P., etc. ..... Augusti. } \\ & \text { Paleacea, Esp. } \\ & \text { Gilvago, Bork. }\end{aligned}$

1162 Caltulago (2). . . . . . . . Russ, mer.

(1) Colore C. Fulvago, et signaturis Abluta afinis; an hybride? Ala antica luteororticina vel luteo-fulve, area media lateritia, maculis ordinariis pallidis, reniformi inferne infuscata, striga extra-basali semi-circulari, magis resulari quam apud Ablutrm. Alos postice lutescentes. - Inventa in Syrmia a D. Kindermann.

(2) Statura C. Fulvago et optime distincta. Alæ anticw luteo-lateritiæ, strigis duabus transversis, ;undulato-serratis, obscurioribus ; macula reniformi patlidiori, foferne infuscata alboque limbata. Alw postica pallide lutex. Subtus ale omnes pallide luted immaculatx. - In Russia meridionali inventa a D. Kindermans. 


\section{GENUS MESOGONA, Boisd}

Mythimua, Boisd., Ochs. olim. Cosmia, Tr.

Larvx olscurx, noctu plantis humilibus vescentes, collo scutellat $x, \mathrm{Ce}$ rastiformes. Metamorphosis hypogaa. - Antenma elongatx, maris crenulato-dentatx, femina simplices. Palpi distantes, breves, horizontales, articulo ultimo brevissimo, nudo, tuberculiformi. Thorax lanatus, rotundatus, antice sub-carinatus. $A$ bdomen carinatum, cylindraceum. Ala latiores, antica arca trapeziformi delincatx; maculis ordinariis nitide scriptis.

\&63 Acetoselle, L., F., H., Tr., D., B. . Gall. cent., etc. Augusti.

1164 Oxalina, H., Tr., D., B........ Germania. . Augusti.

6. GENUS GORTYNA, Ochs., Tr.

Xanthia, Boisd, olim.

Larve elongatx, livide, vermiformes, scutellatæ, in caulibus yel radicibus carnosis occulte degentes. Metamorphosis endophyta. - Antennæ simplices, maris crassiuscula, sub-dentata. Palpi ordinarii. Thorax robustior, antice sub-carinatus. Alæ antic $x$ nitide scripte, maculis ordinaris distinctis. Ablomen feminæ apice conicum.

\section{$\dagger$}

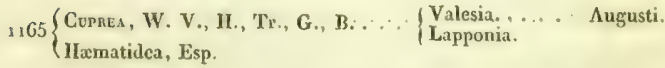

^.66 $\left\{\begin{array}{l}\text { Mrcaces, Esp., Tr., D., B., Curt., Fr. }\left\{\begin{array}{l}\text { Gallia bor., ctc. Augusti. } \\ \text { Anglia. }\end{array} \text { Cypriaca, H. }\right.\end{array}\right.$

\section{斻}

$1162\left\{\begin{array}{l}\text { Flavago, Esp., II., Tr., D., B.. . . Gallia, ctc. . } \\ \text { Rutilago, F. }\end{array}\right.$

$1168\left\{\begin{array}{l}\text { Lumaxa, Kinderm., Tr. ........ } \\ \text { Var. Burcia. } \\ \text { Corsica. }\end{array}\right.$

(Var. Borelii, Pierret, Anu, S, Ent. 

${ }_{1369}\left\{\begin{array}{l}\text { Luteago, F., W. V., H., Tr, D., B. Gall. mer. . . Augusti. } \\ \text { Lutea, Bork. }\end{array}\right.$

Brunneago, Esp.

Var, Olbiena, Donzel, II-G.

\section{GENUS XAN'TIIA, Ochs. , $T_{r}$.}

Larvx scutellatx, glabræ, sordidæ, antice attenuatx, sæpius oblique fasciatx, punctat $x$, folia arborum, rarius plantas humiles pascentes. Metamorphosis subterranea, - (Insecta autumnalia colore flavido rarius ferruginco, ) - Antennæ filiformes, raro maris sub-pectinate. Palpi ordinarii. Thorax rotundatus. Ala flavo variegatx; maculis ordinariis obsolete scriptis.

† Antennx maris sub-pectinatx.

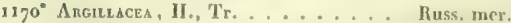

1178 liveccura, Esp., Tr., 13....... Italia.

xigz Miniago $(t) \ldots \ldots \ldots . . . .$. Russ.mer. . Angusti.

t† Antenna simplices.

I173 Evioens, H., Tr., B. . . . . . . . Hungar. ... Augusti.

I174 F Franugines, H., WV. V., Tr., D., B. . P., etc.... Septembr. $1175\left\{\begin{array}{l}\text { Pulmonaris, Esp., Tr., B., Frey.... } \\ \text { Pulmonarix, H. }\end{array}\right.$

Ochrago, $\mathbf{F}$.

Var. Fuscago, Lisp.

Rorina, L., F., II., Tr., I., B. . . . P. P., etc. .... Octobr.

I $\neq 6\left\{\begin{array}{l}\text { Catenata, Esp, } \\ \text { Var. Punica, Bork. }\end{array}\right.$

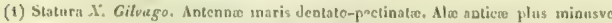
fulvo-rubricantes, macula reaiformi fusca, macula rotunda pallida, oblique posita, strigis transversis pallidis, obsoletis, punctioque sub-marginalibas nigris. Alx posticec albidar, - In Russia ineridionali inventa a D. Kindermann. 
$1172\{$ Asreusta, W. V., F.,Tr., D., B. . . Gall. etc. . Septembr 177 Xerampelina, Esp.

$1178\left\{\begin{array}{l}\text { Xerampeuna }, \text { II., Tr., D., B. . . . . } \\ \text { Monspelii. }\end{array}\right.$ Centrago, Curt. pl. 84 .

1179v.Enтturago (1). . . . . . . . Monspelii.

(Avaco, F., H., Bork., Tr., D., B. . Gall. bor... Octobr.

$1180\left\{\begin{array}{l}\text { Pratexta, Esp. } \\ \text { Var. Rutilago, Bork. . . . . . . German. }\end{array}\right.$

$2181\left\{\begin{array}{l}\text { Silago, H., Tr., D., B. ....... P., etc.... } \\ \text { Flavago, F. } \\ \text { Togata, Esp. } \\ \text { Ochreago, Bork. }\end{array}\right.$

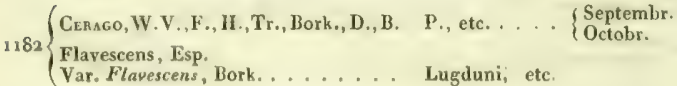

${ }_{11} 83\left\{\begin{array}{l}\text { Gisvaco, F., H., Esp., Tr., D., B. . P. frequens. . } \\ \text { Ocellaris, Bork. }\end{array}\right.$

Var. Palleago, H. 192. . . . . . . P. rarior.

Var, Palleago, Tr., D., H. 443. . . P. frequens.

Ix84 Solphurago, F., W. V., H., Tr., D., B. German. . . Septembr.

1185 Puniceago (2). .......... Russ. mer. . Septembr.

II 86 Crtrago, L., II., etc. . . . . . . P. P., etc. .... Septembr.

(1) Statura Gilvago, Alo antica sub-retusa, apice acuta, fulvo-erocen maculis ordinariis sub-obscurioribus, pallide cinctis; punçtis sub-magibalibus fuscis. Alse posticue pallide ferruginea. - Circa Monspelium a D. Daube detecta.

(2) Statura Sulphurago ; species singularis, iacertxe sedis. Alx anticx luteo-corticino, fascia media angulosa lateritia; strigis duabus pallidis transvernis. Alse postica albils.

- In Russia meridionali inventa a D. Kindermann. 


\section{GENUS HOPORINA, Boisd.}

Xanthia, Tr., Boisd. olim., Dup.

Larva scutellata, glabra, arboricola. Metamorphosis subterranca. Antennx clongatx. Palpi singulares, in rostrum inflexum producti. Thorax rotundatus, antice cristatus. Abdomen truncatum. Dorsum depressum, deplanatum. Alx anticx, basi arcuate; maculis ordinarii, vix distinctis.

1187 Croceago, F., Bork. H., Tx, D., B. . P., etc. . . . Octobr.

\section{GENUS DASYCAMPA, Guenéc.}

Cerastis, Tr., Boisd. olim.

Jarva hirsuta, capite parvo, plantas huyniles pascens. Puppa sub-folliculata. - Antenne maris crassiores, ciliatas. Palpi ordinarii, articulo ultimo gracili. Thorax rotundato-quadratus. Dorsum in utroque sexu deplanatum, depressum. Alx robustiores, nebuloso-intricatx.

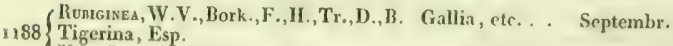
Var. Sulphurago, Bork.

ıo. GENUS CERASTIS, Ochs., Tr., Boisd.

Glrea, Steph.

Larve glabra, obscure, scutellatix, plant is herbaceis noctu vescentes. Metamorphosis hypogaa. - Antenna simplices. Palpi ordinarii, articulo ultimo sub-nullo. Thorax rotundatus. Dorsum in atroque sexu deplanatum, depressum. Alax obscurx, nitidx, maculis ordina. riis obsoletis.-Insecta autumnalia.

$+$

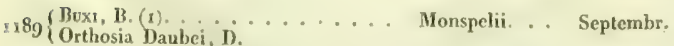

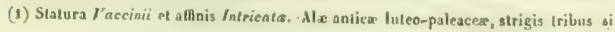


mgo Istricata, B. Ind. Meth. Suppl. ... (Gall mer. . Septembr.

VAccisı, L., W.V., H., etc. (vald. var.). P., etc. . . . Septembr, $1191\left\{\begin{array}{l}\text { Var. Brigensis, Anderr. } \\ \text { Var. Spredece, H. }\end{array} \begin{array}{c}\text { alis longior. } \\ \text { cinereo- } \\ \text { marmorat. }\end{array}\right)$. Valesia.

Var. Ligula, Esp.

Var, Politn, II., W. V, D.

(Dolosa, H., Tr., B. . ........ Hungar. ... Octobr.

$119^{2}\left\{\begin{array}{l}\text { Volos. Crullt, II. } \\ \text { Var. Veronice, } \mathrm{H}\end{array}\right.$

Var. Pulverea, II.

Erythrocephala, W.V., H., Tr., D., 1. Gallia, etc. . S Septembr.

$119^{3}\left\{\begin{array}{l}\text { Vaccinii var, Esp. } \\ \text { Var. Silene, Bork. }\end{array}\right.$

Var, Glubra, WY, V., H., Tr. .... Gallia, etc.

(Sulene, W. V., F., II., Tr., G., B. . Gallia, etc. . Septembr.

${ }_{19}\left\{\begin{array}{l}\text { Vau punctatum, Bork. } \\ \text { C. Nigrum, Devill. }\end{array}\right.$

t+ Alax longiores. Thorax mag. quadrat. G. Mecoptera, Guenée. Scopelosoma, Curt.

1195 Satezlitia, L., F., etc. . . . . . P.. etc. . . Septenbr.

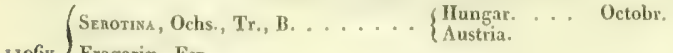

196v. Fragarix, Esp.

Orbona, H., Ross.

Domiduca, Bork.

auatis, obsoletis, fuscis, punctis ad apicem bi-seriatis nigris, maeulis ordinariis vel nultis, vel macula reniformi indicata et inferne nigro infuscata : ale postica fusce, lunula media obscurioci firabriaque Intea. Antenne solito longiores, maris sub-dentalx, - L arvam reperit in Buxo humili Cl. Dauhe circa Alonspelium. 


\section{TRIBUS XYLINIDES.}

\section{Noctude Auct.}

Iarva 16-podx, elongat $x$, stpius concinne colorat $x$, subdiales, in ranis plantarum, vel foliis arborum , sæpius apricantes. Metamorphosis in terwa, folliculata. - $\Delta \mathbf{l}$ insecti perfecti plane tectiformes. Dorsum cristis preditum.

\section{GLNUS XYLINA.}

Xyline, Tr., Boisd. olim.

Larva elongatx, folia arborum, rarius herbarum, per dicm paseentes. Puppa terra folliculata. - Antennæ in utroque sexu filiformes. Palpi ordinarii, articulo ultimo sub-nullo. Thorax latus, plane quadratus, antice sinuatus, medio carinatus. Dorsum deplanatum, cristatum. Ale elongata, angustiores fere ut apud Cucullias. Maculis ordinariis distinctis. - Insecta autumnalia,-Hocce genus ab omnibus aliis facile distinctum thoracis forma.

$$
\text { + Larva herbicolæ. Calocampa, Steph. }
$$

1197 Vexussa, If., Tr., D., B. . . . . P, etc. . . Septembr. 97 Exoleta var., Esp.

${ }_{198}$ Lixoleta, L., etc. ......... P., etc. ... Septembr.

†† Larve arboricolæ.

IIg9 Solnaguns, H., Tr., D., B., Curt. .. \{ $\begin{aligned} & \text { Gall. occid. . . Augusti. } \\ & \text { German. }\end{aligned}$

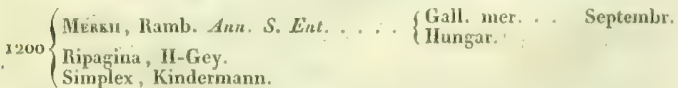

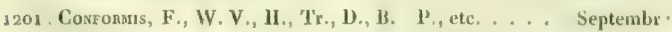

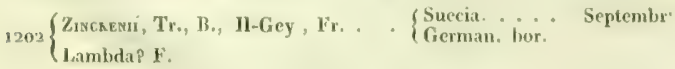


i203 $\left\{\begin{array}{l}\text { Lapidea, II., Tr., B . . . . . . . } \\ \text { Var. Lenutieri, B., Tr., H-Gey. } \\ \text { Var.Sabina, Anderregg, II-Gey, Tr.(1). Valesia, in Sabina. }\end{array}\right.$

1204 Rezonitra, F., W. V., H., Tr, D., B. P., ete. .... Martis.

$1205\left\{\begin{array}{l}\text { P'thificata, F., WV.V.,Bork.,Tr., D.,B. P., cte. .... } \\ \text { Petrificata, H. } \\ \text { Umbrosa, Esp. }\end{array}\right.$

i206 Oculata(2),Germar.Frun.Ins.t.18.Tr. German. etc. . Septembr.

2. GENUS XYLOCAMPA, Guenée.

Xyline, Ochs. Cleophance, Tr.

Jarva elongatie, antice posticeque attenuat $x$, sub-Catocaliformes, inter

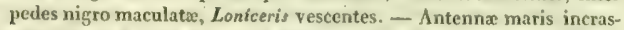
satx, dentatx, basi floccosx. Palpi ordinarii articulo ultimo nudo, distincto. Thorax rotundatus, antice carinato-sinuatus. Dorsum carinatum, cristatum. Maculis ordinariis nitide scriptis.

$1_{207}\left\{\begin{array}{l}\text { Lrmorнiza, Bork., Tr., D., B..... P. frequens. . Martis. } \\ \text { Operosa, II. } \\ \text { Areola, Esp. }\end{array}\right.$

1208 Rasrosa, Esp., H., Tr., D., B. . . : Helvetia. . : Junii.

\section{GENUS CLOANTHA, Boisd.}

Cleopharia, Tr.

Larva lincatæ, Hypericis noctu vescentes, antice attenuate, capite minuto. Metamorphosis hypogaa,-Antennx filiformes. Palpi ordinarii, articulo ultimo vix conspicuo. Thorax rotundato-quadratus, antice sinuato-carinatus, postice penicillatus. Dorsum carinatum, cristatum. Alæ antiex concinne depicte, sub-radiate, macula reniformi nitide scripta, rotunda, minutissima.

(s) A. Sabinct differt ab individuis Galliae meridionalis, liveis moculisque alarum anticarun obsoletioribus. Dom. Anderregs educit larvam in Junipero Sabina.

(2', In multis musxis cum Petrificata confusa, - Alis anticis oblongis angustioribus ferrugineo et fusco-nebulosis, wacula ad angulum internum ovata sub-ocellata, oigra, pallido-eineta. 
1209 Hypericl, F., W. V., H., Tr., D., B. Gallia, etc. . Junii.

1250 Perspicillaris, L., etc. ....... P., etc ... Maii.

$1211\left\{\begin{array}{l}\text { Radross, } \\ \text { Lyncea, } \mathbf{T} \mathbf{r}_{-}, \text {D., B. . . . . . . Hungat. . . Junii . }\end{array}\right.$

\section{GENUS CLEOHHANA, Boisd.}

Cleophana, Tr.

Laryæ Linarias, Scabiosas, etc., per diem pascentes, elongatæ, antice posticeque attenuatæ, lineatæ, virides albo Jineatx, vel albo flavoque lineatæ et nigro punctatx. Puppæ folliculatæ, appendiculo vaginali, gracili, proditx. - Antennx filiformes, maris crassiusculx, varius sub-pectinatx. Palpi hreves, villosi. Thorax rotundato-quadra tus, sæpe penicillis acutis munitus. - Insceta vivida, minora, at robus tiora.

+ An genus proprium $P$

Usturata $(x) ;$ B., H-G. . . . . . Gall. mex. . Junii. $1212\{$ Ustula, Frey.

Caradrina Lurida, Tr. Suppl.

t† An Genus proprium?

1213 v.Crclopea, Graslin, Ann. S Ent. . \{ llispan. mer.

1214 Cymbalare, H., Tr., D., B.....

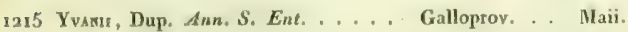

1216 Dejeimu, B., D. .......... Galloproy. . Maii.

1217 Anarghinı, В. .......... Galloprov. . Maii.

1218 v. Penicillata, Ramb. Frun. Audal. . $\begin{aligned} & \text { Hispan.mer } \\ & \text { Galloprov. } \\ & \text { Sardinia. }\end{aligned}$

1219 Anтmkuк1, H., Tr., D., B., Frey. . \{ $\begin{aligned} & \text { Gall. mer. . Jungas. } \\ & \text { Ilunii. }\end{aligned}$

(1) Larva el puppa hujus speciei egre a larva el puppa Antirrhini distingueadue; eam dem Seabiosan simul pascentes ambse harvo I 


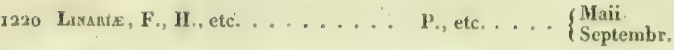

1221 \%.Seurata, Tr. . . . . . . \{ $\begin{aligned} & \text { Sicilia. } \\ & \text { Hispan. mer. }\end{aligned}$

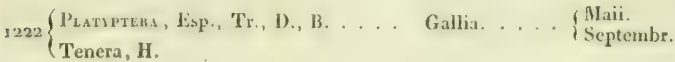

1223 Opsuna, Esp., H., Tr., D., B. . . Gall. mer. . \{ $\begin{aligned} & \text { Miäi. } \\ & \text { Augusti. }\end{aligned}$

122 \} v.Lavders, Anderregg, B. i)..... Valesia. . . Junii.
\end{abstract}

5. GENUS CHARICLEA, Kirby, Steph.

Xylina, Tr., Boisd. olim. Ileliothis, Tr., Suppl.

Latra concinna, Cueulliformis, nigro panctata, flayo-lineata, per dien fructus Delphiniorum mamducans. Puppa conical, terra suls-folliculata. - Antennx filiformes. Lingua elongata. Palpi ordinarii. Thorax rotundatus, antice carinato-sub-acutus, postice penicillatus. Dorsum cristam unicam gerens. Alx roseo-violacea, maculis ordinariis confusis.

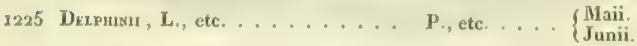

\title{
6. Genus Cucullia, Ochs., Tr., Boisd., Dup., Steph.
}

Larvx concinnx, moniliformes, elongat $x$, flores Syngenesiarum, Verbasia, vel Scrophularias pascentes. Puppx terra folliculate, appendiculo viginali praclita. - Antema filiformes. Palpi ordinarii. Lingua elongata. Thorax rotundatus, antice in cucullum prominulum elevatus. $\Lambda$ bdomen elongatum, cristatum, maris apice bi-penicillatum.

(1) Species pulcherrima, vir major Opalina : alis asticis viveis faseìs tuabus fuscis, externa linea sinuata, albida, divisa; alis posticis albis lunula anedia fasciaque postica nigrescentibus. Corpore toto niveo. - In hosorem D. Laudet Eotomologi eximii Germanice appellata. 
1226 Spectamus, H., Tr., D., B. . . . . Russia mer.

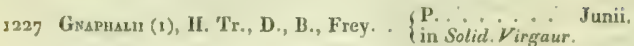

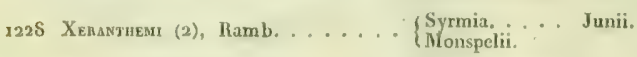

1229 Sastonscr, H., B., D. (3)...... $\begin{aligned} & \text { Valesia.... Junii, } \\ & \text { Dinix, in Artem. Absynth. } \\ & \text { Russ. mer. }\end{aligned}$

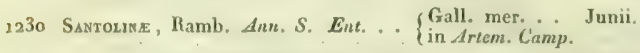

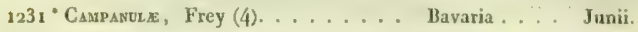

1232 Aвrotanr, W.V.,F.,Bork.,H., Tr., D., B. German. . . \{ $\begin{aligned} & \text { Maii. } \\ & \text { Augusti. }\end{aligned}$

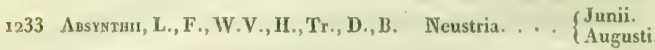

1234 Pontica $(5) \ldots \ldots \ldots$. . . . Russ. mer.

$1235\left\{\begin{array}{l}\text { Antensise, F., W. V., II., Tr., D., B. Germ. bor. . J Julii. } \\ \text { Argentca, Esp. }\end{array}\right.$

1236 M M a

(1) Cucull. Gnaphalii e Russia meridionali discrepat abindividuis nostris, alis anticis ad apicem albidioribus. Cæterum convenit reliquis characteribus cəm specimioibus Gallicis.

(2) Omnioo media inter Cucullias Gnaphalii et Alrotani : forma alarum exacte ut in C. Gnaphalii, signatura vero anticarum fere ut apud sbrotani.

(3) C. Santonici e llussia differt ab individuis larva eductis a D. Anderregg in Valesia, alis anticis angustioribus; cueterum bibil aliod quo dilferat deprehendere possum.

(4) D. Freyer. $N$. Beytr., Tab. 35. Mlanc specien aflinem $C$. Tanaceti ejusque larvau Icone illusirat, illamq̨ue dicit Campanulam rotundifolian manducare. An rite? Larva videtur valde aftinis larva Tanaceti; insectum tanen perfectuin ad Cucull. Santonici Valesie propias accedit cujus larva omoino alia est. Testimonia ulteriora desiderantur.

(8) Statura Artemisic, alue antico cinereo-fusco lineis angulatis nigris plegisque cinereo-albidis. Ala postica cinereo-fusce.

(6) L pulcherrimis. Al antice argentea, nitidissimw, fasciis duabus fuscis punctisque inarginalibus nigris. Also postica niveo punctis marginalibus nigris; thoras niveus peui eillis infuseatis. - In Russia meridionali inventa a $\mathbf{b}$. Kindermann. 


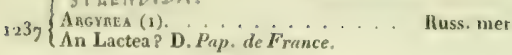

1238 Argentina, F., Bork., H., Tr., B. . Russ. mer.

1239 Lactea, F., Esp., II., Tr., B. . . . R Russ. mer.

1240 Tanacetr, F., W. V., H., Tr. D., B. P., etc. . . . . $\begin{aligned} & \text { Junii. } \\ & \text { Septembi' }\end{aligned}$

1241 Draconculx, H., Tr., D. ...... Russ, mer

$12\{2$ Balsamite (2). . . . . . . . Russia mer.

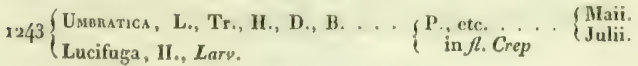

(Chamonile, W.V.,F., Esp., II.,Tr., B. P., etc. . . . J Junii.

1244 $\left\{\begin{array}{l}\text { Var. Chrysan } \\ \text { Lucifuga, D. }\end{array}\right.$

Var, Calendule (4), Dahı. ..... Sicilia.

Var.P Leucrnthemi, Ramb. Faun. And. Gall. cent.

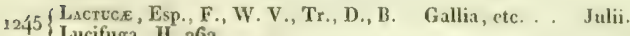

Lucifuga, II. 262.

1246 Lucragu, Esp., W. V., B. . . ... In Alp. freq. . Juli.

1247 Virgauke $(5) \ldots . \ldots \ldots$. . . . Russia mer.

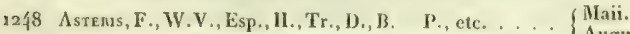

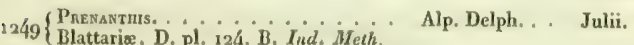

(1) Statura Argentina. Alse antica tote argenteo-micacea, viridi-sub-micantes, frabrie alba, margine tenui ia illosis croceo. Ala postica albidw, ad extimum fuscescentes fimbria alba.

(2) Statura Dracunculi. Ale antice cinerese ntomis albidis immixtis, linea basali, medio, longitudinali, nigra, nitide scripts, fulvo limbala. Als posticw fusca disco al bido, fimbria nivea. - In llussia meridionali inventa a D. Kiodermana.

(3) Obscurior individuis Germanicis, at larva endem.

(4) Dilutior individuis Chamomillo Europe centralis. Larva iisdem characleribus signata, at multo pallidior et fere paleaces.

(8) Valde affiois Asteris at distincta. Also antica apice magis acuta, costa unargineque interno obscurioribus, arcu albido sob-nullo, maculis ordinariis inconspicuis, Cxclerum fere ut apud $C$. Asteris, - Inventa in Rosuia meridionali a D. Kindermann. 
t+ Larv. Scrophul, aut Verbasc, pascentes.

125o Scrophuzariphaga, Ramb........ Corsica.

in Scrop. ramosissim.

1251 Tuapstphaga, Tr., D., B., Ramb. . \{ $\begin{aligned} & \text { Gall. mer. . Maii. } \\ & \text { in } V_{\text {erb. }} \text { romosis. }\end{aligned}$

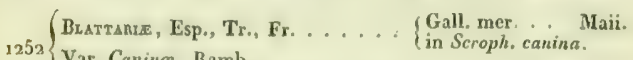
Var. Caniure, Ramb.

(Var.P Scrophularivora, Ramb. F. And.

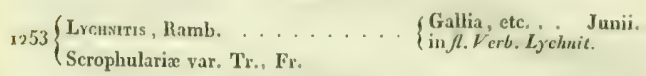

1254 Scrophuqume, Ramb., H., Esp.P. . $\left\{\begin{array}{l}\text { Gall.cent, ete. Maii. } \\ \text { in Scroph. aqunt, ct nod. }\end{array}\right.$

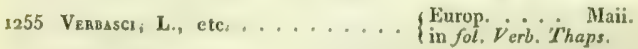




\title{
XXV. TIRIBUS CALPIDES.
}

\author{
pseudo-bombycini, Boist., olim.
}

Larve 16-podx, clongatir, shabra, sub-monilifornes, plantas humiles pascentes, flavo lineatx nigroque punctatx.-Metamorphosis in folliculo laxo. P'appa conica, - Antennx maxis sub-pectinatx, feminx crenatx. Palpi crassi, compressi, longiores, porrecti. Lingua mediocris. Thorax rotundatus, transversim undulatus. Dorsum leve. $\Lambda$ la latiores, tectiformes. Anticx exterue sinuate, margine interno late appendiculatx.

1. GLNUS CALP'E, Tr., Boisd.

Calypera, Ochs.

Larva forma, more, picturaque hocce genus Cucullias revocat; insecto perfecto, ad $I /$ usias prope areedit. Finte quadam species $A m e r i c s:$ borealis, habita Plusice, ad Calpides attiment.

$1256\left\{\begin{array}{l}\text { T'incris, Bork., H., Tr., B., D. . . \{ l Pyren. or. } \\ \text { Capucina, Esp. }\end{array}\right.$ 


\section{TRIBUS PLUSIDES.}

Larva tenue sub-pilosula, semi-Geometre, perlibus ventralibus primi paris ad progressum inaptis, vel frequentius, pedibus duodecim tantum proditx. P'uppa inter folia folliculata. Alæa acute tectiformes. Dorsum eristatum.

1. GENUS ABrostolı, Ochs., Boisd., Tr. Suppl.

Larvæ 16-pedibus preditx; yentralibus primi paris brevissimis, ad progressum inaptis, plantas sub-herbaceas per diem pascentes. l'uppar folliculatx. - Antenna simplices. Palpi ultra frontem assurgentes, articulo ultimo gracili, elongato. Thorax rotundatus, postice penicillatus. $\Lambda \mathrm{l} x$ fusc $x$, maculis ordinatiis squamulosis.

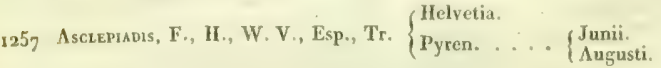

258 Untıce, II., Tr., B. . . . . . . P., etc. . . . \{ $\begin{aligned} & \text { Junii. } \\ & \text { Augusti. }\end{aligned}$

1259 Triplasia, L., 11., etc. ....... P., ete.... $\begin{aligned} & \text { Junii. } \\ & \text { Augusti. }\end{aligned}$

2. GENUS GHRYSOPTERA, Latr., Boisd., Dup.

Larva 12-poda, Thalictris et Aconitis vescentes. Puppx folliculatx, discolores. Antennx maris crassiores.-Palpi ultra frontem valde assurgentes; articulo ultimo clongato, arcuato, ciliato. Thorax villosus, cristato-penicillatus. Alx latiuscula, auro plagiato-marmoratw.

$1260\left\{\begin{array}{l}\text { Deaunata, Esp., Tr., B., D. ..... \{. \{ } \begin{array}{l}\text { Valesia. } \\ \text { IIungar. }\end{array} \\ \text { Aurea, H. . Julii. } \\ \text { Chryson, Bork. }\end{array}\right.$

$1261\left\{\begin{array}{l}\text { Moneta, E, Bork., H., Tr, D., B. . } \\ \text { Flavago, Esp. }\end{array}\right.$

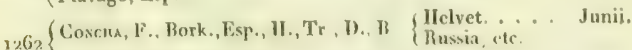
C. Aurcum, Knocls. 
3. GEIVUS PLUSIA, Ochs., Tr., Lat., Boisd.

Larvx 12-pedibusinstructx, herbas pasentes. Puppe folliculatx, sxpius discolores. - Antenne simplices. Palpi ordinarii. Lingua exserta. Thorax villosus, postice valde penicillatus, robustior. Dorsum cristatum. Al: metallicæ, sæpius aureo vel argenteo inscriptx. Maculis ordinariis nullis.

$\dagger$

1263 Consosi, F., Bork., II., Tr., D., B. . Hungar. . . \{ Junii.

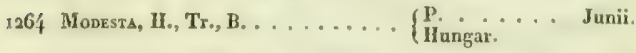

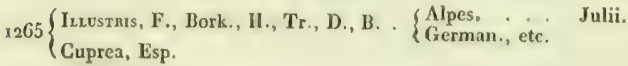

$+t$

${ }_{1266}\left\{\begin{array}{l}\text { Mrs, II., Tr., Frey. . . . . . . . } \\ \text { V. Argenteum, Esp. }\end{array}\right.$ $1267\left\{\begin{array}{l}\text { Cnussıtıs, II., Tr., D., B. ..... } \\ \text { Chalcites, Bork. } \\ \text { Bengalensis, Rossi. }\end{array}\right.$

x668 Qoestionis, 1I., Frey, Tr., Ochs., B. Lusitania.

J269 $\left\{\begin{array}{l}\text { Accestiren , Lefebv., B., Tr., D. } \\ \text { L. Aureum, Frey, tab. 23. } \\ \text { Hieroglyphica, Frey, p. 176. } \\ \text { L. Album, H-Gey, 856. }\end{array}\right.$

s27o Festuce, L., etc. ........... P., etc..... \{ $\begin{aligned} & \text { Junii. } \\ & \text { Augusti. }\end{aligned}$

$1271^{\circ}$ Zosıs1, H. 651 . . . . . . . . I Ilispan. mer.

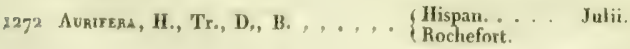


${ }_{1273}\left\{\begin{array}{l}\text { Cunnsiris, L., cte. . . . . . . Europ. . . . . } \\ \text { Var. A. (fasciis aureis coadunatis). }\end{array}\right.$

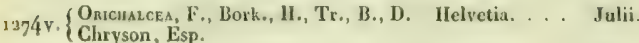

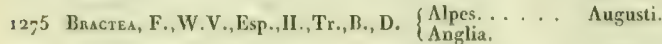

Ainou, W. V., HI, Tr., B. . . . . Styria. . . . A A gusti. 1276 Lamina, F., Bork.

Chrysomelas, Bork.

1277 Cracunscbipta, Dahl., Frey, Tr. . . S Sicilia,

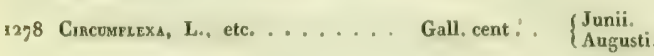

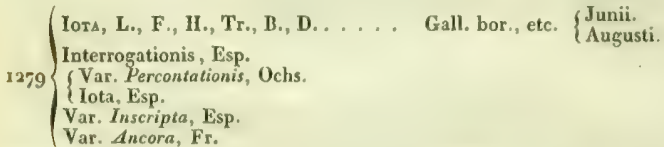
$1280^{\circ}$ Enea, H. . . . . . . . . . An Europæa P

\section{$+t+$}

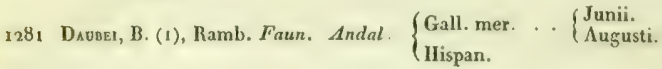

1282 Gamsı, L., ctc.. . . . . . . . Europ. . . Perannat.

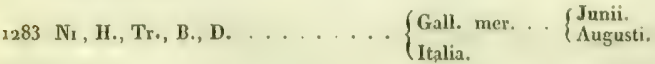

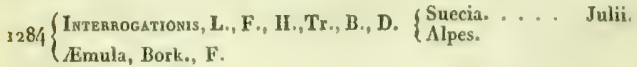

1285 v.PARLIS, H., B. Russia.

(1) Pl. Ni paulo minor: alis anticis cinereo-fuscis, nitidis, linea argentea tortuosa, in disco litteram $\mathbf{Y}$ quasi formante, loco macule reniformis arcu argenteo angustissimo signatis ; posticis pallide cinereis, apice infuscatis. - Nominata in honorem D. Daube qui hanc speciem circa Mtosspelium invenit.-Cl, doctor Rambur in Hispania quoque reperit ot e larra specinina nitidissima eduxit. 
1286 Drasem, Dalm., B., J). . . . . . . Lapponia. . . Augusti.

1287 Micnograms, H., Tr., B., D. . . . German, bor. . Julii.

1288 Aur, Lsp., Rork., 11., Tr., B., D .. Alpes.. . . . Augusti.

1289 Devergens, II., Tr. . . . . . . Mpes. . . . Augusti

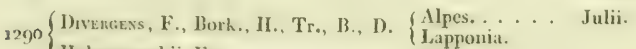

(Iolenwarthii, Esp. 


\section{TRIBUS IIELIOTIIIDES.}

\section{Noctua Auct.}

Larva clongatx, 16-pod $x$, subdiales, frutices vel plantas liumiles depascentes. Puppx folliculatx, vel terra sepulta, - Insecta vivida thorace robustiori, per diem volitantia, etc.-Ala tectiformes. Dorsum lreve.

\section{GENUS ANARTA, Ochs., Tr., Boisd.}

rarvx glabriuscula, punctatx, arbuscula pascentes. Puppe folliculatx. - Antennx elongatx, setiformes. Palpi hirsuti, breviores. Thorax robustus, dense villosus, rotundatus. Abdomen ablureviatum, conoideum. $\Lambda$ la breviores, robustx, maculis ordinariis, sxpius obsoletis. Alæ posticæe, coloratæ.

1291 Mүктиц, L. etc.......... P., etc.... Maii.

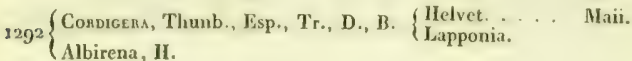

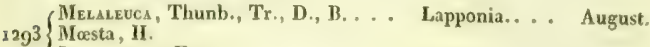

Leucoptera, Esp.

1294 Algids, Lefebv. Ann. S. Ent. . . . Scandinavia.

1295 v.Asussa, Lefebv. Ann.S. Ent. . . . Reg. pol.

$1296\left\{\begin{array}{l}\text { Mielanores, Thunb., B. . . . . . . . . } \\ \text { Vidua, H., Tr., D. } \\ \text { Var. Tristis, II. }\end{array}\right.$

1297 v.Funebris, H., Tr., D. . . . . . . Helvetia.

1298 v Nıgrata, Anderregg ( 1 . . . . . . Hẻlvetia. . . Junii.

(1) Alis nigris funbria alba; anticis inacuta reniformi obsoleta, fasciaque inarginali repanda, cincreis. An aberratio Melenope P - Funcbris IJ, rarissima, et individua lusa trata hujus speciei in miseis DU. Donzel et Pierrel haud exacte converiunt cum fig. Hubneri, quocirca facile credatn omnia aprcimina unica, scilicel, Funebris H., Funebrit Pierret, Funebris Donzel et Nigrita Anderregg, tanlum aberrationes Melanopa. 
1299v. $\left\{\begin{array}{l}\text { Ropicona, W. V., Ess., Tr., D., B. . Hungar. ... Junii. } \\ \text { IIfliophila, H. } \\ \text { Pallium, Bork. }\end{array}\right.$

(Anетт, F., D., B. ........ P., ete..... Maii.

$1300\{$ Heliaca, H., W. V., Tr.

(Fasciola, Esp.

Note. Anarto porpusilln, B, Ind, Meth, Suppl, ad Genus Ennychia referenda.

\section{GENUS ANTHORCIA, Boisd.}

Meliothis, Tr.

Jarve elongata, punctatx; capite parvo, globuloso, floribus seminibusque Syngencsix vescentes. Metamorphosis in calycibus. - Antemnx simplices. Palpi breves, articulo ultimo abortivo. Statura parva, robusta. Thorax rotundatus. Abdomen plane conicum, annulatum; oviductu feminæ exserto, acuto.

1301 Cognata, H-Gey., Frey, Tr. .... Hungar..... Junii.

i3o2 Cander, Esp., H., Tr., D., B., Frey. A Austria, etc. . Junii.

3. GENUS HELIOTIIS, Ochs., Tr., Boisd.

Larva elongate, antice posticeque attenuatr; capite parvo, pilis geminatis sat distinctis instructæ, plantis altioribus vescentes. Puppe terra scpultx.-Antennx simplices. Palpi ordinarii, hirsuti, articulo ultimo inconspicuo. Thorax rotundatus, robustior. Dorsum læve, conoideum. Ala robusta; postica nigro marginatx; antica subtus lu nula fasciaque nigris.

+ An Gen. proprium ? $3303\left\{\begin{array}{l}\text { Fravaldsıкrı, D. . . . . . Turcia. } \\ \text { Viola, Frey., H-G. }\end{array}\right.$

$+t$

3301 Oross, F., W.V., Esp., H., Tr., D., B. Gall, or., ete. . $\begin{aligned} & \text { Maii. } \\ & \text { Augusti. }\end{aligned}$ 
1305 Dlpsacea, L., etc. ........ Europ. ... \{ $\begin{aligned} & \text { Maii. } \\ & \text { Augusti }\end{aligned}$

1306 Scurosa, F., Esp., 11., Tr., B., D. . Gall. m., etc. J Julii.

13om $\left\{\begin{array}{l}\text { Peltigera, W. V., H., Tr., D., B . Fallia mer... Junii. } \\ \text { Scutigera, Bork. }\end{array}\right.$

Barbara, F.

Florentina, Esp.

1308 Amugers, II., Tr., D., B. . . . . Gall, etc. . Augusti.

$\left\{\begin{array}{l}\text { Marennata, F., Tr., D., B. . . . . Gall, etc. . Junii. } \\ \text { Rutilago, W. V., II. }\end{array}\right.$

Umbrago, Esp.

Conspicua, Bork.

${ }_{1310}$ Ponpunutes (1), Tr., D., B., H-Gey . . Hungar. ... Maii.

Rutpurea, Esp.

33 I Boisouyalit (2), Ramb. Fran, Andal., Ilispan, mer.

Var. Incarnatn Kinderm. ....... Russ. mer.

\section{GENUS HABMEROSIA, Boisd.}

Anthophiln, Tr.

Larva elongatx, antice attenuate, capite parvo, glabriusculæ, punctatæe, plantas herbaceas pascentes. - Puppæe terra, sub-folliculatæ. Antennæe maris crassiores, crenata, femina simplices. Palpi ordinarii, capite longiores; articulo ultimo gracili, brevi. Thorax rotundatus, squamulosus, Dorsum læve. Abdomen conicum.

+ An Gen. proprium ?

\$312 Commonamaculs, F., H., W.V., Tr ,D., B. Ilungar... . . Augusti.

(1) Purpurites ab Hel. Marginata nisi colore purpureo vis differse videtur, Characteres alaram el larve a D. Freyer depicto in Scabiosa succisa, fere omoino conveniunt cum Marginala, An varietas P

(2) Statura Marginata : ala antica virescenti-lutescentes costa fimbriaque rubris, unaculis ordinariis t'uscis, reviformi albo signata. Alw postiew, cinerex, pallida, nigro margiaatie, fubrin rubsa; capite thoraeeque purpureis. I.arva frequens in Silenibus viscosis Ilispanive meridionalis. - Hombur.

Specimina e liussia meridionali differunt, alis anticis viridi-hrrbaceis, in sitene ameria lacvam reperit 1). Kimferinann, circa 'Taganrok. 
$+t$ $1313\left\{\begin{array}{l}\text { Renitera, Boisd. } \\ \text { Pyr. Renalis, H. }\end{array}\right.$

Monspelii. .. Maii.

131 Albicans, Ramb. Faun. Andal. . . Mispan. mor. tt+ An hujus generis?

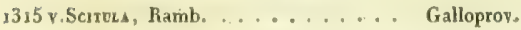




\section{TRIBUS ACONTIDES.}

\section{Nocture Auct.}

Iarva clongatx, geometrx, pilis rariusculis prxditx, plantas agrestes pascentes. Puppre terra sub-folliculatæ. - Insecta diurna, vivida. Alx omnes althe, rarius lutex; fusco nigroque maculat $x$ vel fasciata. Antenna simplices. Palpi breves, squamoso-pilosi. Thorax rotundatus, squanosus. Abdomen conicum, læve. Alæobtuse tectiformes.

^. GENUS ACONTIA, Ochs., Tr., Boisd.

+ Alæ flavæ.

1316 Matve, Esp., H., Tr. B. . . . . Sicilia.

13 i v. Graelsn, Feisth. Ann. S. Ent., H-G. Catalaunia.

t+ Alæ albæ.

1318 v.Aprics, H., Tr., B. . . . . . Italiamer.

1319 v. Cenustua, Frank, Tr., B....... A Andalusia.

1320 Calons, H., Tr., B. . . . . . . Græcia.

${ }_{1321}$ Titania, Esp., Tr., B. . . . . . . Russ. mer.

${ }_{822}\left\{\begin{array}{l}\text { Sorınis, W. V., Bork, H., Tr, D., B. P., etc. . . . A A Augusti. } \\ \text { Albicollis, F. }\end{array}\right.$

$323\left\{\begin{array}{l}\text { Luctuosa, W. V., H., B., D. . . . . P., etc. . . . \{ } \begin{array}{l}\text { Julii. } \\ \text { Augusti. }\end{array} \\ \text { Italica, F., Devill }\end{array}\right.$

Nola, Dom. Curtis (British Entomology), sub nomine Acontia Cutena, 1ab. 276 , speciem pulchellam illustrat, ut hospitem anglican. Iloc insectum mihi videtur Americanum et ad nostrum Genus Eudryas forte referendum. 


\section{TRIBUS CATOCALIOES.}

Noctuce Auct.

Larva elongatx, antice posticeque attenuata, folia arborum, vel arbustorum pascentes, per diem sxpius ad ramos immobiles et cum corticibus confusa, vel ad radices arborum, sub quisquiliis latentes; Geometriformes, 16 , rarius 14 pedibus gaudentes. Pedes ventrales, antici breviores et ad progressum inapti. Puppe folliculatx, rarius ferra sepultæ. - Antenne simplices, maris rarissime pectinatæ. Alis obtuse et late tectiformes, per quictem triangulum referentes.-Volatus fere sub-diurnus.

\section{GENUS CATEPIIIA, Ochs., Tr., Boisd.}

Iarve pseudo-Geometre. Puppe folliculatx, - Antennx simplices Palpi ultra frontem valde assurgentes, articulo ultimo elongato, nudo, acieulari. Thorax dense villosus. Dorsum valde cristatum. Ala nigro-fuscæ, disco posticarum albo.

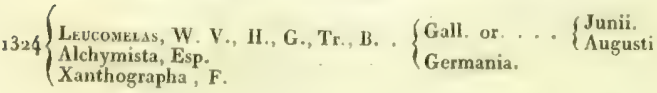

$1325\left\{\begin{array}{l}\text { Ramburn, 13., Tr. . . . . . . . . } \\ \text { Adepta, H. } \\ \text { Leucomelas, Clerk. Icones. }\end{array}\right.$

${ }_{1326}\left\{\begin{array}{l}\text { Alcursmsta, F., W. V., JI., Tr., G., B. P., ett. . . . . Maii. } \\ \text { Leucomelas, Esp., Naturf. }\end{array}\right.$ 
x. Genus Catrocala, Ochs., Tr., Boisd.

Larvæ vividx, subsilientes, arboricolæ, fusco-cinerex vel cinerex, subtus depress $x$, inter pedes maculate, lateraliter fimbriatx; sxpius dorso tuberculatic. Puppa cxsio-pruinosx, inter folia folliculatx. Antenna longx, simplices, maris rarius sub-crenulata. Palpi mediocres, articulo ultimo brevi. Thorax rotundatus, levis. Abdomen elongatum, conicum, basi obsolete cristulatum, maris apice penicillatum. Alæ latiores; anticæ obscuræ; postic rubræ, luteæ, vel cæruleæ, nigro bi-fasciata, rarissime unicolores.

+ Carulex.

1327 Fraxin, L., etc, ........ P. etc. .... Augusti.

t+ Rubre.

(Llocata, Esp., Bork., Tr, G., B. . G Gall. mer., ete. Augusti.

1328 Uxor, II.

Nupta, F.

Var. Nurus, H-Gey., 655.

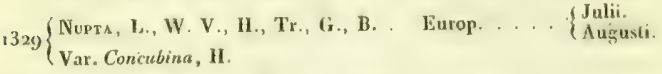

$1330\left\{\begin{array}{l}\text { Dilecra, H., Tr., Bork, I3.(an var.sequ.P). Gall. mer. . Julii. } \\ \text { Sponsa var., Gr. }\end{array}\right.$

$33 .\left\{\begin{array}{l}\text { Sponsa, I., F., H., Tr., G, B. ... P., etc. .... } \\ \text { Ab. Rejecta, Fisch. (posticis fusc. fascia nigra). }\end{array}\right.$

332 $\begin{aligned} & \text { Pnomssa, F., Bork., W. V., IX., Tr., B. Europ..... } \\ & \text { Sponsa var, G. } \\ & \text { Var. Mneste, II. }\end{aligned}$

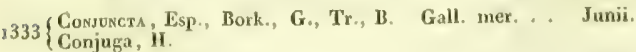

334 Optata, G, B., Tr. . . . . . Gall. cent. . Juiir.

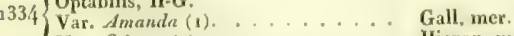

Var. Selecta (2). . . . II . . Ispan, mer.

(1) Alue anticx obscuriores, postica vividius coccinex.

(2) Differt a varietate Amanda abdomine rubro. 
$1335\left\{\begin{array}{l}\text { PАстА, L., F, H., Tr., G., B. . . } \\ \text { Pacta Suecica, Esp. }\end{array}\right.$ $1336\left\{\begin{array}{l}\text { Electa, Bork., H., Tr., G. . . . G Gall. ctc. . . } \\ \text { Pacta, W. V., Esp. }\end{array}\right.$

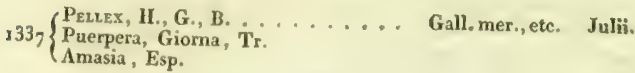
tit Lutex.

3338 Neonmpha, Esp., H., Tr., B. . . Lithuania, etc. Julii. ${ }_{333}\left\{\begin{array}{l}\text { Nrmpнz, , Esp., Bork, H., Tr., B. . Galloprov. . J Julii. } \\ \text { Var. Vestalis, B., H-Gey. }\end{array}\right.$ 1340 $\left\{\begin{array}{l}\text { Conversa, Esp., Tr., G., B. . . . Gall. mer., etc. Julii. } \\ \text { Pasythea, H. } 455 .\end{array}\right.$ 13 \{1 $\left\{\begin{array}{l}\text { Agaros (1), H., Tr. . . . . . . A } \begin{array}{l}\text { Austria. } \\ \text { Pall. occid. }\end{array} \\ \text { Pasythea, H. 338. Julii. }\end{array}\right.$ 1342 Paranrapan; L, F, etc. ....... P., etc. ... Julii. $13\left\{3\left\{\begin{array}{l}\text { Nrmpragoga, Esp., II., Tr., B. . . G Gall. mex. . . Julii. } \\ \text { Nymphæa, God. }\end{array}\right.\right.$ ×344 Disjoncra, HI-Gey., B. ...... Dalmatia.

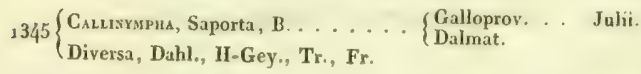

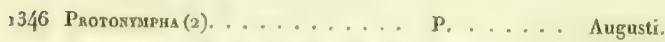
$1347\left\{\begin{array}{l}\text { Hrsen.eA., F., W. V., H., Tr., G., B. Hungar. . . . Angusti. } \\ \text { Var. Posthuma, H. }\end{array}\right.$

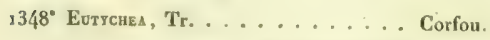

(1) Agamos qua cirea Burdigalam occurrit difert a Conversa genuina, alis posticis valde obscurioribus, subtus pracipue, margineque externo albido.

(2) Species distinctissima antennis crassioribus dentatis; statura Callinymphe; alia postice fere ut apud jam dictam; antica cinereo-fusce fascis vix angulosis. 
3. GENUS OPIIUSA, Ochs., Tr., Boisd.

Larva pseudo-Geometræ; sxpius corticina, 16, rarius 14 pedibus gaudentes, folia arborum, vel arbustorum, rarius, plantas humiliores pascentes. Puppa folliculatx, vel terra sepulte. Antennæ simplices. Palpicontigui, ultra frontem plus minusve assurgentes, articulo ultimo nudiusculo, sat producto. Thorax rotundatus, antice sub-sinuatus. Abdomen longiusculum, dorso lævi. Alx, pro corpore, magrix, maculis ordinaris, modo distinctis, modo inconspicuis. Alx posticæ, sæpius uti anticæ coloratæ.

\section{$+$}

2349 $\begin{aligned} & \text { Trknnxa, F., Cram., G., Tr, B. . Gall. mer. . . } \\ & \text { Auricularis, II. }\end{aligned}$

Vesta, Esp., Bork.

Lunaris, F., W. V., II., G., Tr., B. . P., etc. . . . Junii. $1350\{$ Meretrix, $\mathbf{F}$.

Augur, Esp.

t† Leguminos, herbac. pascentes.

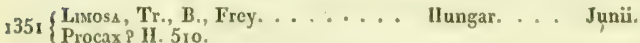

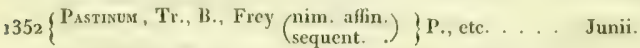

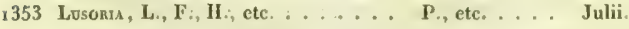

¿354 v. Astragad1, Ramb. Frun. And. . . Hispan. mex.

\355 Ludicna, H., G., Tr., B. . . . . Ilungar. . . Julii.

${ } 356$ Овови, B............. Hungar.

\357 Vrcie, H., Tr., B. . . . . . Gall., etc. . Julii.

1358 Cracce, F., W. V., II, G., Tr., B. Gall., etc. . Julii.

\section{$+t+$}

3359 v.Rectanguramis, H-Gey., Tr. . . . . Dalmatia.

1360 Illuenaris, Hi, G., Tr., B. . . . . . Gall, mer. . . Julii. 


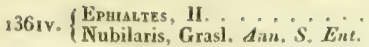

Hispan, mer,

\section{$+十+\dagger$}

1362 $\left\{\begin{array}{l}\text { Punctularis, H., Tr. (1)....... Russ. mer. } \\ \text { Repanda, F. }\end{array}\right.$

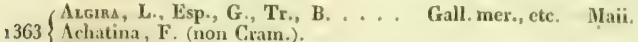

Triangularis, $\mathbf{H}$.

$1364\left\{\begin{array}{l}\text { Geometrucs, F., G., Tr., B..... Gall. mer! . Julii. } \\ \text { Parallelaris, H. } \\ \text { Ammonia, Cr., Esp. } \\ \text { Chalciptera, Bork. }\end{array}\right.$

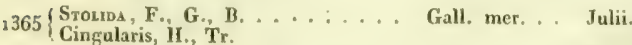
1366 Canixo, Lefebv., B. . . . . . \{ \{ Gall. mer. . Julii.

\section{GENUS MCROPHISA.}

Ophiusa, Tr.

Larva ignotx, verisimile Geometriformes,-Antennx simplices. Palpi mediocres, clypeosub-longiores, squamato-hirsuti, contigui, articulis sub-aqualibus. Thorax rotundatus, debilior. Abdomen cylindricum, apice conicum. Alæ vix teetiformes, patulæ.-Statura minor. - Volatus diurnus.

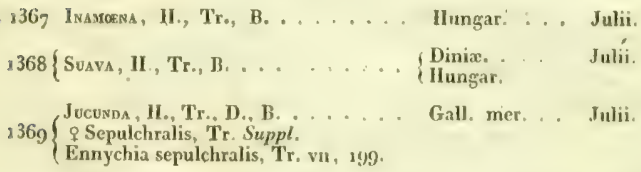

(1) Punctularis H. certe eadem species ac Repanda tab.; habitat sat frequens in Ben. salia, insulis Africe, et America. Nulla vidi specimina ia Europa eapta. Colore variat, smper vero distiocla pedibus maris dilatatis, remiformibus. 
1370 Regurahis, H., Tr., B. . ...... Russ. mer.

137 Ineqularis, II., Tr., B.(1). . . . . An Europ.?

\section{GENUS CEROCALA, Boisd. \\ Ophiusa, Tr.}

Larva ignota. - Antennx maris clongate, pectinatx, femina filiformes. Palpi ultra frontem valde ascendentes; articulo ultimo elongato, gracili, aciculari. Lingua exscrta. Thorax rotundatus. Abdomen elongatum. Alæ latiores.

$13 \eta_{2}$ Scapolosa, II., Tr., D., B. . . . A Andalusia.

(1) Omnia specimina lustrata ex Amnrico meridionnli orta. 


\section{TRIBUS NOCTUOPHALANIDES.}

Larva Geometrx, plantas humiles, vel folia arborum manducantes. Metamorphosis sul-terranea, vel sub-folliculata, Insecti minora. Volatus diurnus. Ala plas minusve tectiformes.

\section{GENUS EUCLIDIA, Ochs., Tr., Boisd.}

Larva elongatx, Ophiusiformes, strigosx, pedibus 12 tantum gaudentes, plantas humiles (Leguminos. presertim) pascentes. Puppa sub-folliculate. - Antenux breviores, simplices. Palpi breviores, articulo ultimo gracili. Thorax mediocris. Abdomen lave sub-glabrum. Ale robustiores; antica figuris Geometricis signatæ; postica, et omnes subtus, coloratx. - Volatus diurnus.

$13 \eta^{3}$ Fontatiliom, 11., $\mathbb{B} \ldots . . .$. Russ. mer.

${ }_{13} 34$ Mr, L., etc. ........... P., etc. ... Junii.

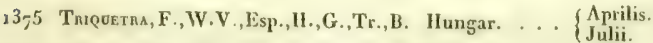

ı 376 v.Munits, H., B. . . . . . . . Russ, mer,

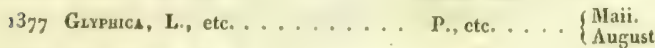

з378 Monocrangra, H., G., Tr., B. . . . Gall. met.

2. GENUS BREPHOS, Ochs., Tr., Boisd.

Larvæ pseudo-Geometra, inodice clongatx, strigosx, arboricola, filopendule Geometrarum instar; pedilus ventralibus quatuor anticis abortivis, ad progressum inaptis. Puppa tenue folliculata. $-\mathrm{An}$ tenne maris sub-pectinatx, vel in utroque sexu simplices. Palpi mediocres, valde villosi. Thorax mediocris, villosus. Abdomen cylindraceum, lxve, villosum. $\Lambda$ lx rudiuscule squamosic ; posticx, et omnes subtus, luter, nigro fasciatac.-Volatus meridianus. 
+ Antennx maxis simplices.

$1379\left\{\begin{array}{l}\text { Partuenias, L., H., etc. . . . . P. P., etc..... Martis. } \\ \text { Vidua , F. } \\ \text { Notha, Curtis, tab. 221. Steph. }\end{array}\right.$

计 Antennæ maris pectinatx.

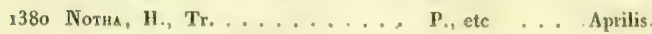

${ }_{138}\left\{\begin{array}{l}\text { Poelza, Esp., Bork., Tr., B. . . . G German. ... } \\ \text { Spuria, H. }\end{array}\right.$

Parthenias var., G.

3. GENUS TIMIA, Boisd.

Larra ignota. - Antennx maris pectinatx, feminæ dentata. Palpi brerissimi, fronte breviores, contigui, articulis indistinctis, Lingua brevissima. Thorax mediocris, lanatus. Abdomen conicum, læve. Alæ purpurex auro argentoques ignatæ. - Genus omuino incertæ sedis : forsan ad Bombyces referendum. - Metamorphoses desiderantur.

1382 Mangarita, H., 13......... M llonspel. . Junii.

4. GENUS ANTHOPHILA, Boisd.

Anthophile et Erastriae, Ochs., Tr.

Larvæ Geometræ, pedibus 12 gaudentes, plantas Syngenesire sæpius pascentes. - Arstennæ setaceæ, mediocres. Palpi contigui, compressi, longiores, ultra frontem assurgentes, articulo primo breviori, secundo elongato. Thorax rotundatus, minutus. Abdomen cylindricum, elongatum, lave. Alæmediocres, debiliores. Antice absque maculis ordinariis. Pedes postici longiores. - Volatus diusnus. Insecta minuta.

3383 P'urpunana, F., WV. V., 11, Tr., D., B. Galloproy. . A Agusti. Yar.9 Rosina, 11. 
1384 Ostrina (1), H., Tr., Curt., B. .... $\begin{aligned} & \text { Gall. mer. . } \\ & \text { Hispan. }\end{aligned}$

$1385\left\{\begin{array}{l}\text { ExeA, W. V., Bork., H., Tr., D., B. P., etc. . . . Julii } \\ \text { Latruncula. }\end{array}\right.$

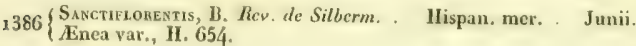

1387 Krnderamanku (2)........... Syrmia.

1388v. $\left\{\begin{array}{l}\text { Vespentısa, Tr., B......... } \\ \text { Pyr. Vespertalis, H. }\end{array}\right.$

1389 Glarea, Tr, H., Frey, B. . . . . Gall, mer. . Julii.

${ }^{3} 3 g \circ\left\{\begin{array}{l}\Lambda_{\text {moexs }}, \text { Il., Tr., B. . . . . . Gall, mer. . } \\ \text { Respersa, Bork. }\end{array}\right.$

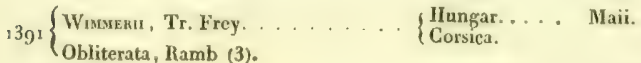

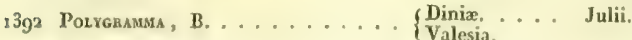

1393 Concinnula (4). . . . . . . . . Russ. mer.

${ }_{1394}$ Paula, H., Tr., B. . . . . . . Gall. mer. . Junii.

1395 Erycurysr, Ramb. Ann, $S$, Ent. . . Corsica, . . . Junii.

${ }_{13}{ }_{9} 6$ PARVA, HI., Tr, B. . . . . . . . Gall. mer. . Junii.

${ }_{1397}{ }^{3}$ Mnuta, H., Tr, B. . . . . . . . Gall. mer. . Junii.

${ }_{1398}$ Pora, H., B. .......... \{ $\begin{aligned} & \text { Gall. mer. } \\ & \text { frequens. }\end{aligned}$

$1399\left\{\begin{array}{l}\text { Argentela, Bork., Esp., Tr., D., B. . P. . . . . . Junii. } \\ \text { Pyr. Bankiana, F. }\end{array}\right.$ (O)ivea, $\mathbf{H}$.

(1) Specimina estivalia valde variant, signaturis obsoletis, colore paleaceo vel albicante

(8) E puleberrimis. Statura Purpurina, Also antica ad basin late nitiderue flava pubctis tribus minutis nigris, dein cinereo-fusca striga obsoleta albida; posticie fusca.

(3) Nomen Hamburianum antiquius certe, sed rejicieadum cum jamdiu apud Dalman speciem Lapponicam indical.

(b) Paulo inajor Paula. Ala antico albido-glaucescentes fasciis duabus albis, una inedia geniculata, altera terminali. Posticae albue sub-fuscercentes. - In Russia meridjanuli a D. Kinderman detreta. 


\section{GENUS AGROPHLA.}

Anthophile et Erastria, Ochs., Tr.

Iarve semi-Gcometre, plantis humilibus vescentes. Puppa folliculatx. - IIabitus Tortricum, vel Pyralidum.-Antennx setiformes, breviores. Palpi brevissimi, hirsuti, contigui, articulis indistinctis. Lingua elongata. Thorax rotundatus, robustior, minutus. Abdomen lxve. Alæ debiliores. - Genus hocce Tortrices tangere videtur.

† Maculis ordio. nullis.

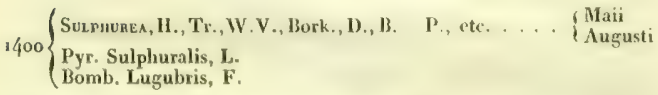

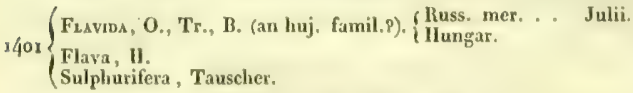
t† Maculis ordin. nitidis.

(UNCA, W. V., Esp., Bork, D., B. . P., etc. . . . Junii. 1 /402 Pyr. Uncana, F.

Geom. Uncana, L.

\q03 Numertca (1), . . . . . . . Cotsica.

\section{GENUS ERASTRIA, Boisd. Erastria, Ochs., Tr. Acosmetia, Curt.}

Larva semi-Geometra, plantas humiles vel arbusculas pascentes. Puppa inter herbas folliculata. - Antennæ filiformes, clongata. Palpi clongati, frontem ultra assurgentes, graciles, articulo ultimo, brevi aciculari; secondo clongato, horizontali. Thorax rotundatus, minutus. Abdomen elongatum, in utroque sexu cristatum. Statura debilis. Alæ latiusculæ, maculis ordinaris nitidis. - Quod genus ad Gcometras jam protendit.

(1) Statura Evantric Fuscule. Alw anlice fusen, strigis tribus angulatis niveis; muculis ordinariis nitide seriptis, niveis, nomerum 80 figurantibus, Ale postice pallidat ad apicem obsolete fusco-bifasciatx. In dumehis Corsicas detesit CI. Cantener. 
$1404\left\{\begin{array}{l}\text { Fuscura, W. V., Bork., II., Tr., D., B. P., etc. . . . . Junii } \\ \text { Polygramma, Esp. }\end{array}\right.$

$1405\left\{\begin{array}{l}\text { ArnatelA, Bork., W. V., II., Tr., B. P., etc. . . . F M Maii. } \\ \text { Tineodes, View. }\end{array}\right.$

\406 Casdidera, W. V., Bork., II., Tr., B. Gall., etc. . Junii.

1,̧07 Parvora, Ramb. (an huj gener.P). . Galloprov.

1 f̧08 Venostors, II., Tr. ........ Gallia. .... Julii.

7. GENUS STILBIA, Steph.

Caradrina, Tr.-Ophiusa? Boisd. Ind. Meth.

Larva ignota. - Habitus Geometra. Caput solito crassius. - Intennæ maris ciliato-dentate. Palpi brevissini, sub-remoti. Lingua producta. Thorax lavis, rotundatus. Abdomen gracile, licve, sub-elongatum. Ale tectiformes, sub-debiles, ampliores, maculis ordinariis oblique positis. - Genus incert $x$ sedis.

(Stagmicola, Tr. (non D., B.). .... Gallia.... Scptembr. Lathyri, B. Ind. Meth.

$\$ 409$ Anomalata, Ilaworth, Ent. trans.

Geom, Ilybridata, $\mathbf{H}$.

Oph. Anomala, Ingpen, Instruct. gr. 


\section{GEOMETR A Auctorum.}

Characteres familix : larve 10 , rarius 12 pedibus tantum instructæ. Antennæ maris modo simplices, modo plus minusve pectinatix; ferminx semper setiformes. Palpi inferiores semper occultantes superiores, villosi, sxpius breviores. Alx integra, minus robusta, pro corpore magnx, per quietem horizontales, rarius obtusissime tectiformes vel erecta.

$\mathrm{Si}$, Noctuarum turbam tractando, non dissimulavimus quantæ nobis difficultatis fucrit insecta in seriem continuam et naturæ consentaneam disponere, hîc fateri debemus Geometras coordinare nobis longe majoris diflicultatis opus visum fuisse, et corum generum quxcumque assumpsimus aut constituinus iu illa sectione, pleraque multo magis etiam vacillare superioris familix Generibus. Ea difficultas, procul dubio, tum ex negligentia oritur, quia longo temporis spatio illa insecta minus diligenter a superiorum xtatum auctoribus explorata sunt; tum ex penuria characterum organicorum quos perfecta ostendunt. Itaque, postquam multa experti fuimus incassum, et frequenter pretentavimus, tunc solummodo, nec sine labore, potuimus species in quosdam greges cogere, quos genera nominavimus. Verum haud potuimus communes characteres reperire, ut in illis primarias sectiones, alio verbo Tribus, constitueremus.

DD. Treitsclıke, Stephens, Curtis, et recentius Duponchel, labores synonymiam liujus Heterocerorum partis non mediocriter quidem extricaverunt. Sed dicere ne vereamur, corum virorum ordinationem duntaxat inchoatam et adumbratam rem esse, ut multa desiderentur : nos tamen haud confidimus multo majus effecisse. Utilitatem cepimus non minus ex collationibus quas illi feliciter invenerunt, quam ex erroribus qui (absit verbo invidia) illis exciderunt. Ita opinamur nos universam classificationem aliquanto in melius emendavisse, sed noster labor nobis tandiu videbitur imperfectus, donec diligenter exploratx metamorphoses cjus rationem comprobaverint.

Animadvertetur etiam, et fortasse aliquis nonnihil mirabitux, nos, audaces novatores, omnes species ordinatudo, quaslibet yoces, seu intennx simplices sint, seu plus minusve pectinatx, syllabis arin uniformiter terminavisse. Quodquidem necessitatem induxit nomina quxelam mutandi; sed rectius omnes Geometre in consimilem fincm desinent, ut Pyralides, Tortricides et Tincides. 


\section{GENUS IIELIOTHEA, Rambur.}

Iarva ignota, - Corpus robustius. Palpi brevissimi, villosi. Lingua sub-nulla. Antennæ crassiuscula, rigidiuscula, maris pectinatx. Alæ angust $x$, robustiores, unicolores, puncto majori discoidali fimbriaque nigris. - Volatus meridianus.

1410 Discoidara (1), Ramb, , F. And. . Hispan, mer.

\section{GENUS CLEOGENE, Dup. Minoa, Treits.}

Larve minus elongatx, torulosæ, gibbosæ.-Antennæ maris pectinatæ. Palpi breves, villosi. Lingua sat producta. Alæ rotundatæ, unicolores. Statura media. Femina mare minor, per diem quiescens in herbis.

- Volatus maris diurnus in montosis et alpibus.

+ Ala lutex.

3411 Tinctarla, II., Tr., D. . . . . Alpes...... Julii.

Lutearia, Esp., F.

t+ Alx albidx.

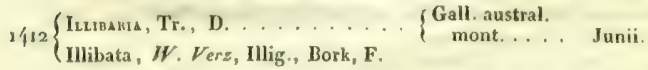

tt? Alæ nigræ.

- Hand strigatæ.

Iq13 Peqetienaria, D., H-Gey...... Pyren. ... Julii.

$\because$ Striga transversa obsoleta.

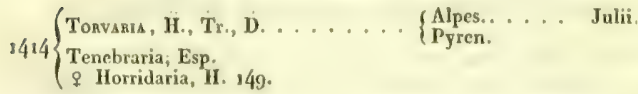

(1) Spartiarin vis minor, alis angnstioribus, utrinque lutris, inargine punctoque majori nigris. 
3. GENUS GLOMETRA, Boisd.

Geometra, Tr., Dup:

Liarva breviuscula, torulosa, Notodontiformis, dorso gibbosi. Metamorphosis folliculatia. - Antenw maris pectinatx. Palpi ultra clypeum assurgentes, articulo ultimo nudo. Lingua brevis. Alæ virides, denticulatæ. Statura magria. - Volatus crepuscularis:

1 i 15 Paphlonaria, I. etc........ \{ $\begin{gathered}\text { Europ. bor. et } \\ \text { cent. }\end{gathered}$

Junii.

\section{GENUS PHORODESMA.}

Geometre, Tr. Geometra et Hemithea, Dup.

Litrva breviusculx, capite rotundato, dorso mamillares, bajularix. Me tamorphosis folliculata.-Antenna maris pectinata. Alæ virides. Statura media. - Volatus crepuscularis.

Notri. Hocce genus more et habitu larvarum paybhidis revocat:

×416 Smaragdaria, Esp., H., Tr., Fr. . . Bavaria,... . Junii.

Basularia, Esp., W. V., II., Tr., D, P. etc. ... Junii. $1417\left\{\begin{array}{l}\text { Ditaria, 1. } \\ \text { Pustularia, Panzer. }\end{array}\right.$

5. GENUS IEMITHEA, Dup.

Geometrix, Tr.

Larre rigidx, laves, capite bifido, segmento primo spinis duabus inclinatis instructo. Metanorphosis sub-folliculata. Puppa sxpius punetulatx. - Antenna maris pectinatw. Pilpi graciles, clypcum superantes Lingua mediocris. Ala virides (rarius cinerex), integra; postica sappius angulariter productie. 
† Alx posticæe rotundatæ; fimbria unicolor.

- Virides.

Crrmsaria, W. V., Esp., H., Tr. . P........ Junii. Prasinaria, F. 1418 Genistaria, Devill., D.

Var. Coronillaria (1), D. (non H.).

Var. Agrestaria, D.

1419v.Porraceara, Ramb. ........ Monspelii.

\section{- Cinerex.}

1420 Corsicanl , R. Ann. S. Ent. . . . . Corsica. . . Maii.

3431 Cononilnara, H., Tr. (2), Ramb. . \{ $\begin{aligned} & \text { Corsica, } \\ & \text { Hispania. . . Maii. }\end{aligned}$

If Alæ posticæ angulatæ, virides.

- Fimbria unicolor.

$1422\left\{\begin{array}{l}\text { Vermar1a, W.V., H., Tr., D. .... Gall. cent. . . } \\ \text { Chrysoprasaria, Esp. }\end{array}\right.$

$1423\left\{\begin{array}{l}\text { VıRIDARIs }, \text { H. D. . . . . . . . Gallia. .... } \\ \text { Viridata, L., F. Tr. }\end{array}\right.$

1424 Crorekr, H., Tr., D. . . . . . Gall, austr. . Junii.

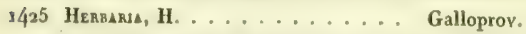

I426 ERdGiNaru, W. V., H., Tr., D. . . A Austria, . . . Maii.

1427 Putatakia, L., H., etc. . . . . . . P., etc. . . . Junii.

"* Fimbria intersecta.

$1428\left\{\begin{array}{l}\text { Estrvaria, Esp., H., Tr. . . . . . P., etr...... Junii } \\ \text { Thymiaria, W. V. } \\ \text { Vernaria, F. }\end{array}\right.$

(1) In Gallia castrali occurrunt identidem specimina cineres, primo intuitu Coronillaria fere similia, sed a Corsicaria et Coronillaria genuina facile distincta, fronte fusco sigaturisque omnino ut apud $C_{y}$ thisariam.

(2) Cl. Treitschke have speciem in genere Gnophos locando erravisse ecosemus,

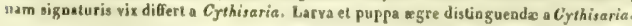



$1429\left\{\begin{array}{l}\text { Bupuevraria, W. V., F., H., Tr., D. . P., etc. .... Julii. } \\ \text { Thymiaria, Lin. }\end{array}\right.$

It Alæ posticæ rotundatæ; fimbria rubra.

$1430\{$ Indigenara, Devill., Tr., D. . . . . Gall. mer. . Maii.

Fimbriolaria, $\mathbf{H}$.

6. GENUS METROCAMPA, Latr, Dup. Ellopia, Treits.

Larva elongatæ, arboricolæ, 12-podx, Catocaliformes, lateraliter fimbriatæ. Metamorphosis folliculata. - Antennæ maris pectinatæ. Palpi graciles, vix ultra clypeum assurgentes. Alæ patulæ, rotundata, vel angulatæ, virides, vel corticinæ, bilineatæ. - Statura magna. - Volatus sub-diurnus.

+ Alx rotundatx.

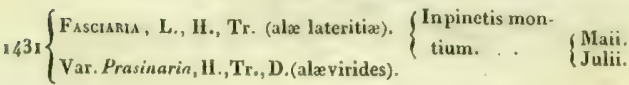

tt Ala angulata.

$1432\left\{\begin{array}{l}\text { Makgaritara, L., H., etc. ..... P., etc. ... } \\ \text { Boml. Sesquistriataria, Esp. }\end{array}\right.$
$1433 \begin{aligned} & \text { Aprilis. } \\ & \text { Julii. } \\ & \text { Excisaria, Esp. }\end{aligned}$

7. GEINUS URAPTERYX; Kirby.

Ourapteryx, Leach., Steph., Dup. Accena, Ts.

Larvæ elongatæ, lateraliter tuberculatæ, capite depresso. Motamorphosis folliculata. - Antennx maris pectinate (in nostratibus sub(iliatæ). Palpi brevissimi. Lingua producta. Alx patulæ, albæ, vel sulphurex, transversim lineat $x$; antic $x$ ad apicem acut $x$; postic $x$ angulato-caudatæ. - Volatus crepuscularis. 
1434 Persicania, Mẻnétriés. Catal. Rais. : : $\begin{aligned} & \text { Caucasas. } \\ & \text { Sarepta. }\end{aligned}$

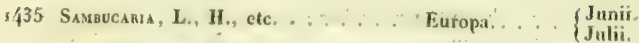

\section{GENUS RUMia, Dup. \\ Ennomos, Tr.}

Larva elongati, torulosa, tuberculata. Metamorphosis folliculata. Antennx maris ciliatx, feminæ simpliciores. Palpi breves, articulo ultimo exiguo. Lingua elongata $A l x$ rotundatx; posticx ad angulum ani sub-productx; anticx aul costam macula ordinaria, fere Nopeurum instar signatx. - Volatus crepuscularis.

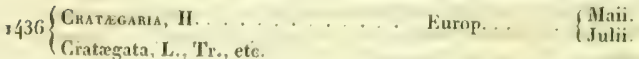

\section{GENUS ENNOMOS, Dup. \\ Ennomos, Tr.}

Larva modice clongata, nodulosie; capite depresso, emarginato. Mc. tamorphosis sub-folliculata. - Antenna maris pectinatic. I'alpi ultra dy yeum sub-assurgentes. Thorax robustior, lanatus. Ala inæqualiter dentatx, Junulata, vel tantum transversin lineato. Statura media vel magna. Ala per quietem sæpius erectæ.

+ Alx sinuate

- Antica sub-integra.

1/37 Svringama, L., II., etc. . . ...... P., etc. . . . M Maii.

1438 Dolabraria, L., etc. ....... P., cte..... M Maii.

" Anticx emarginatx.

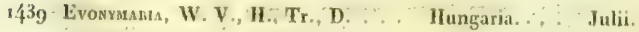

1440 Conpiaria, II. Tr. . . . . . . . Hungaria.... Julii.

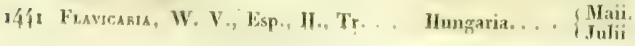


t† Alæ sinuato-sub-emarginatæ. G. Epione, Bup.

1442 Aprcians, W. V., Esp., H., Tr:, D. P., etc.... Julii.

J43 Parallelaria, W. V., II., Tr., D. . P., ete. . . J Julii. $144^{3}$ Vespertaria, $\mathrm{F}$.

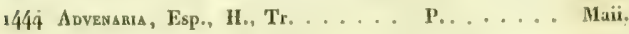

3445 Pectinama (x), II , Tr., D. . . . An Lurop. 8

t† $\Lambda$ l $x$ dentato-eros $x$.

- Antica lunulatx.

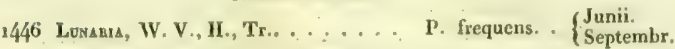

1447 Delunaras (2), II. Steph. . . . . . P. frequens. Junii.

1472 Lunaria, D.

$1448\left\{\begin{array}{l}\text { Illunanı, WV. V., H., Tr., D. ... P., etc. .... } \\ \text { Var. Minor (Estivalis), D. pl. } 144, \text { f. 3. }\end{array}\right.$

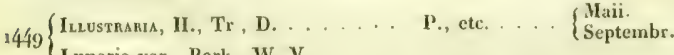

Lunaria var,, Bork., W. V.

- Anticx transversim lineatæ, delunatæ.

1458 Erosaria, W, V., H., Tr., D. . . . . P., ete. .... Junii.

452 Quercinarua, Bork., II. ,Tr. .... Gall. bor. .. J Julii.

1453 Qoencsms, II., Tr. . . . . . . Alsatia..... Julii.

1454 Tiliakia, H., Tr., D. . . . . . . P...... Augusti.

1455 Azmaria, L.; H., etc. ....... P., etc. . . A Augusti.

Dentakia, Esp., II., Tr., D. ..... P., etc.... Maii.

^456 $\left\{\begin{array}{l}\text { Bidentata, L. } \\ \text { Bidentaria, F. }\end{array}\right.$

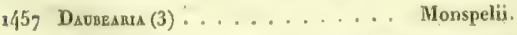

(1) Specimina mes ex America boreali orta?

(2) Certe species plane distincta.

(3) Statura Alniaria; alto dentata, nitida, cinerexe, atomis obscurioribus, margine fuscescente, lineis transversis obsoletis, Feminam tantum novi. 
tt+f Alx posticne dentatx; anticx integra, G. Angerona, Dup.

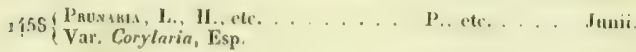

10. GENUS HIMERA, Dup.

Crocallis, Tr.

Larva laves, rigida, elongatx; capite rotundato ; segmento penultimo bi-mucronato. Metamorphosis sub-terranea. - Antenna maris plumosa. Palpi villosi, clypeo breviores. Thorax robustus, lanatus. Alw sub-denticulata, puncto centrali; anticx lineis duabus trapezium includentibus signatx. Statura magna, robustior. - Volatus nocturnus.

1459 Pexnaria, L., II., ttc......... Europ. .... Octobr.

11. GENUS CROCALlis, 'Tr., Dup.

Larva torulose, elongatx, capite crassiori, arboricola. Metamorphosis in terra vel sub quisquiliis. - Antennæ xaris pectinata. Palpi ultra clypeum assurgentes; articulo ultimo gracili. Lingua vix ulla. Thorax lanatus, robustus. Alx lutea, sub-denticulatx puncto centrali; antica lineis duabus trapezium includentibus signatæ. Statura magna, robustior. - Volatus nocturnus.

1460 Ехтикали, II., Tr., D. . . . . . \{ Galloprov.

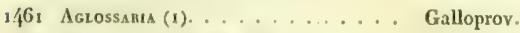

${ }_{3462}$ Llanguafua, L., H., etc. ...... P., etc.... Augusti.

I. 603 Trapezaria(2)............. Monspelii..... Junii.

(1) Statura Pennaria, media inter Extimariam el Elinguariam, Alis rufescentibus, ferrugineo irroratis, anticis saturatioribus strigis, duabus pallidis, maculaque media nigra, punclata.

(2) Minor Elinguaria, colore pallidiori, lineis duabus Iransversis magis divergentibus ; punetis marginalibus nullis. Larva rugulosa, obscuve ferruginea, segmento penultime uni-cuspidato, - Circa Monspelium detesit D. Danbe. 


\section{GENUS SCODIONA. \\ Fidonia, Tr., Dup.}

Larvx elongatx, lxves. Metamorphosis sub-terranea.- Antenrıx maris longiores, angustiores, pectinata. Pulpi brevissimi. Lingua subnulla. Thorax villosus, minus robustus. $\Lambda l_{a}$ cinerex, intererrimæ, puncto centrali; antica atomis irrorata, lineis duabus interruptis, punctularibus trapezium includentibus signatx. - Statura majuscula.

1464 Conspensaria, W. V., F., H. Tr., . . Hungaria.

465 Tonturama (an var. proced.?). ... Diniæ...... Julii. 1465 Conspersaria, Dup.

1466 Lemorscama, Donzel, Ann. S. Ent. . Monspelii.

1467 Emucidaria, H., D. .......... Gall. mer. : J Julii.

$1468\{$ Faviluacearis, H., Tr., D. . . . . Gallia. . . . Junii

Var. of Belgiaria, $\mathbf{H}$. 1469 Penspensaria (1), D., Tr. Suppl. . . \{ $\begin{aligned} & \text { Monspelii. . Septembr. } \\ & \text { Hispania. }\end{aligned}$

\section{GENUS AVENTIA, Dap.}

Ennomos, Tr. Platypteryx, Lasp.

Larva depressa, Gatocaliformis, lichenivora, lateraliter fimbriata. Metamorphosis sub-folliculata. - Antenna maris pectinata. Palpi sat breves. Lingua producta, Alx antic late emargimata; postic rotundatæ. - Habitus Platypterygis.

Frexulanı, II., Tr., D. . . . . Gallia. ... Julii.

$1470\{$ B. Flexula, F., Bork., IV. V.

Platyp. Flexula, Lasp.

(1) Mirandum sane Cl. Duponchel hic retulisse figuran Respersario Hubueri, et descriplionem Gnoph. Perspersata D. Treitschke qua ad speciem omnino diversain perlinent: - vido p. $200, n^{\circ} 1500$. 


\section{GEIVUS MACARIA, Curtis, Steph. \\ Ennomos, Tr. Philobia, Dup.}

Larvæ lieves, elongatx, antice attenuatæ; capite parvo, cordiformi. Metamorphosis sub-folliculata, vel intra quisquilias. - Antenna maxis modice pectinatx. Palpi breves, apice contigui. Thorax debilior. Alx antice emarginat $x$, vel sub-truncat $x$; posticx angulosx. Statura sub-media, debilior.

+ Alx antica valde emarginatæ.

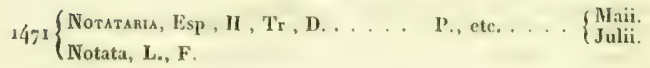

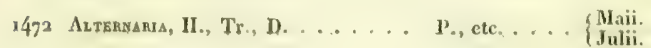

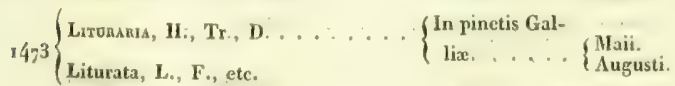

t+ $\mathrm{Mlx}$ anticae vix emarginatæe.

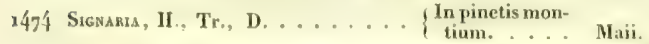

\section{GENUS GODONELA.}

Eimnomos, Tr. Philobia, Dup.

Larva elongata, lavis; capite parvo, rotundato, viridis, allo maculatostrigata, Tamariscicola. Metamorphosis subterranea.-Antenna maris simplices. Palpi contigui, mediocres. $A$ lx antice, cinerex, margine obscuriori; posticæ angulosæ.

Hoc genus plures species Africanas comprehendit; facile distinctum a Macariis et Haliis structura antennarum.

3475 Wistimaria, 1., T $\mathbf{r}_{*}$, D. ........ 
16. GENUS HALIA, Dup.

Fidonia, $\mathbf{T r}$.

Jarva clongata, tenue pilosiusculx. Metamorphosis sub-folliculatı. - Palpi breviusculi. Lingua porrecta. Alx cinereæ; anticx obsoletc, sinuatim lirieatæ, maculis tribus costalibus fuscis.

$\dagger$ - 1 le postice sub-angulosx.

$: 476$ S tevenahita (1) . . . . . . . Russia mer.

f† Alx posticx rotundatx.

1477 WАyaus, L., H., etc. . . . E Europ. . . Julii.

\section{GENUS ASPILATES, Tr. \\ Aspilates et. P'cllonia, Dup.}

Larva herbivore, flabrx, laves, filiformes, valde elongata, sxpius serpentium instar se volventes. Metamorphosis sub-folliculata. - Antenne maris pectinatx. Palpi longiores. Lingua producta. Pedes longiusculi. Alx tectiformes, integrx; anticx striga unica obliqua , yel strigis duabus signatx; posticx rotundata. Statura media. Volatus diurnus.

† Alæ omnes rubro strigatæ. G. Pellonia, Dup.

1478v. Cruentaru, II., Tr., D. . . . . . Mispania.

1479 Vibicarta, L., H., etc. . . . . E Europ. ...

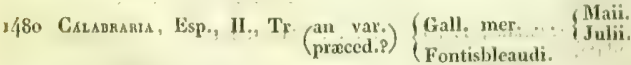

(1) Major quan Wavaria, Alic cincreo-nitida, striga basali alieraque intra medium et apicem angulosa, communibus, fuscis. Antica maculis tribus costalibus nigro-fuscis. Postice ungulariter producte. - firca Blisabethgrart a Ci. Steven, rt recentius in Ilispania meridionali a Doct. Rambur, ioventa. 
" Alæ postica haud strigatæe.

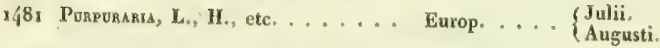

1482 Sanguimaru, Ramb. Faun. And.... Malaga.

t† Alæ anticæ rosex, striga flava.

1483 Rosearra, Tr., D. . . . . . . . $\begin{aligned} & \text { Russia mer } \\ & \text { Dalmat. }\end{aligned}$

1484 Consecraria, Ramb., F. And. .... Hispan. mer. . Julii,

t+† Alæ anticæ flayæ.

- Striga rubra.

1485 Anthophraria, H. (an spec. distinct.P). Russ. mer.

I486 S Scraru, L., F., II., etc. . . . . . E Europ. austral. Junii.

"Striga fuscx.

$1487 \mathrm{Y}$. Gesticdiaria, H. . . . . . . . Russ, mer.

$1488^{\circ}$ Tennana, Fr. . . . . . . Dalmatia.

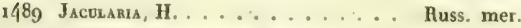

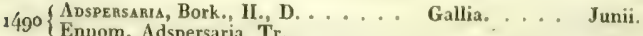

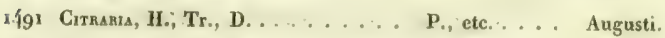

1492 Gilvaria, W. V., H., Tr., D. . . G Gall. mer. . A A gusti.

t+十† Alæ fuscæ, strigatæ.

$149^{3 \mathrm{v}}$. Betricaria, Ramb., F. And. . . . I Iispan. mer.

t+†+† Alæ antic $x$, argenteo-fasciatæ.

149 f Glomosaria (1). . . . . . . . . Palud. Somonæ. Junii.

$149^{5}\left\{\begin{array}{l}\text { Mundatarua, Cram., Tr., D. . . . R R Russ. mer. } \\ \text { Nitidaria, F, Borkh. }\end{array}\right.$

(1) Statura Plumistarice. Also anticx flava puneto nigro, fascia obliqua alteraque marginali margaritaceis; postice albida fascia fusca. 


\section{GENUS THETIDIA}

Jarva ignota. - Antennæ maris pectinata. Palpi brevissimi, contigui, articulis vix distinctis. Lingua vix ulla. Thorax rotundatus. Ala tectiformes, integerrimx; anticæ nervis strigisque argenteis; posticæ immaculatæ. Statura majuscula, - Volatus diurnus.

Ilocce genus plures species $A$ fricanas et unam tantum ex Europa australiori comprehendit.

1496 Plusiaria (1), Ramb. F. And.... \{ llisp. m.

19. GENUS LIGIA, Dup.

Fidonia et Aspilates, Tr.

Larve elongata, laves, segmento penultimo cuspidato, Dorycnium pascentes. Metamorphosis sub-folliculata. - Antennx maris pectinatoplumosæ. Palpi brevissimi. Lingua vix ulla. Thorax villosulus. Frons cucullata. Alæ integræ, tectiformes; anticæ angustiores; posticx immaculatx. - Volatus crepuscularis.

Hoc genus a Gencribus vicinis facile differt cucullo valde producto insecti perfecti.

1497 Jodrdanaria, Tr., D., H-Gey. . . . G Gall. austral. . Septembr. .. x/198 Opacarua (2), H., D., Ts. Suppl.... \{ Gall. mer. . Septembr

(1) Statura paulo minor Jourdanaria. Ala antica late virides, nervis strigisque angulatis argenteis ; postice albida. - Ad idem genus attinet Geometra Frosinaria. Stoll, pl. $36,1,7$.

(8) Larva hujus speciet a larva pracedentis agerrime distinguitur. 
ao. GENUS PLOSERIA

Fidonia, Tr. Numeria, Dup

Larva depressa, cinerea, nigro variegata, Populicola. Metamorphosis folliculata. - Antenna maris simplices. Palpi breviusculi. Lingux brevis. Statura majuscula. Alæ anticæ pulverulentx, lincis duabus transversis, externa sinum magnum ambiente; striga apicali obliqua; alæ posticx lutex.

Iloc genus a Vumeriis et Fidoniis facile distinctum structura antennarum. $1499\left\{\begin{array}{l}\text { Drvensanı, H. . . . . . . . Germai. bor.. Aprilis. } \\ \begin{array}{l}\text { Aurantiata, F. } \\ \text { Diversata, Tr. }\end{array}\end{array}\right.$

21. GENUS NUMERIA, Dup.

Fidonia, Tr:

Larvæ tenue tuberculos $x$, antice attenuatæ, arboricola. Metamorphosis sub-folliculata. - Antennæ maris pectinatx. Palpi breves. Lingua brevis. Statura majuscula. Alx anticx pulverulenta, lincis duabus transversis, externa sinum magnum ambiente; striga apicali obliqua.

$\$ 500$ Pulverara, L., H., etc. . . . . Reg. subalp. Julii.

150 I Capheolara, F., W. V., H., Tr. ... Alp. provin... Julii.

1503 v. Donzelaria, D., I1-Gey. . . . . A Arvenia. . . Iugusti.

\section{GENUS FIDONIA.}

Fidonia, Tr., Dup.

Larva elongatæ, læves, capite rotundato. Metamorphosis sub-follicu. lata vel sub quisquiliis. - $\Lambda$ ntennx maris late plumosx. Palpi breves, villosi. Lingua brevis. Caput haud cucullatum. Thorax villosus, robustior. Alx patulx, integerrimx, sæpius concinne depicte; posticæ sæpius discolores. Statura major. - Volatus diurnus.

+ Alæ omnes fuscre

1503 y Miniosaria, D. . . . . . . Gall, bor. 


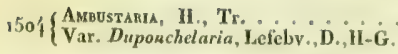

1505 Temolabia, H., D.

Sicilia.

Monspelii... Octobris.

Fontisbleaudi.

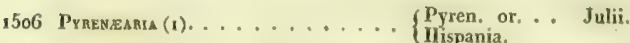

(Pluararia, W. V., II., Tr., D. .... Gall. mer. . Julii. 1507 Vespertaria, Esp.

Var. 9 Limbatn, Ramb. Ann. Sc. Phys.

Var. P'enuulaturia, $\mathbf{H}$.

$1508\left\{\begin{array}{l}\text { Semicanaria, Fr., Tr. } \\ \text { Panormaria, Dah!. }\end{array}\right.$

Sicilia.

1509 Osrmark, Ramb, F. And...... Hispania mer.

†† Alæ anticæ atomis irroratæ.

1510 Pimlaria, L., H., etc. .......... In pinetis Gal-

Aprilis.

1511 Pennigeraru, II., Tr., D. .....

${ }_{1512}$ Ghrustuana, H. . . . . . Sicilia.

$1513\left\{\begin{array}{l}\text { Melanahis, L., H., etc ..... . . I llelvet. . . Junii. } \\ \text { Zerene Melanaria, Tr. }\end{array}\right.$

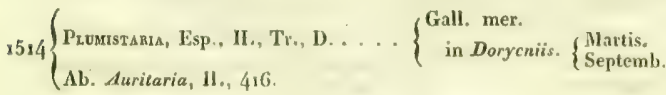

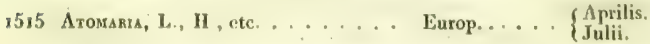

(1) Statura et affinitas Phumarie, Ale fusco-rubricantes, ad spicem sub-olscuriores, fascia nedia nigra, lineaque sub-terninali undulata albido-cinerpa. Ale anticas maculis duabus costalibus fuscis, abruptis, vet in fascias obsoteto desinentibus. 


\section{GLNUS EUPISTERIA}

Fidonice, 'T'r., Dup.

Larvæ elongatx. filiformes, leves. Metamorphosis sub-folliculata - Antenna maris angustius pectinate. Palpi villosi, breviusculi. Iingua sub-producta. Thorax debilior. Alæ semi-patulx, integerrimæ; antice lineato-atomarix; posticæ sæpius ut anticæ depictæ. Pedes breviusculi. Statura minor, vel sub-media. - Volatus subdiurnus.

\section{$+$}

1516 Concondara, II., Tr., D. ...... P., etc.... Junii.

5 (Piceana, H., Fr., Tr. . . . . . Valesia.

2517 (Var. Roscidaria. II. . . . . . Lapponia.

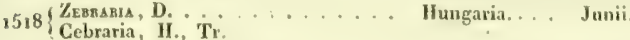

tt

.519 $\left\{\begin{array}{l}\text { Qungoania, II., D. . . . . . . . } \\ \text { Pinetaria, Tr. } \\ \text { } \text { Pinetaria, H. }\end{array}\right.$

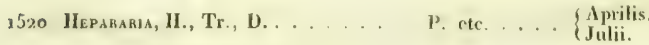

\section{GENUS SPERANZA, Curtis. Fidonin, 'T'r, Dup.}

Larva læves, lineata, clongatx, Genistivora, ad ramos appressas. Metamorphosis hypogæa. - Antennæ maris angustius pectinata, ipice mucronatx. Palpi breviores. Thorax robustior. Pedes breves. Ala integerrima, lutex, nigro limbatie. Statura sub-media, - Volatus diurnus. - Alx per quictem erectx.

1521 Conspicuara, Eisp., Bork., II. Tr. .. Sylv. Gall. bor. Junii.

Limbaria, F., D.

Rorania, Esp.

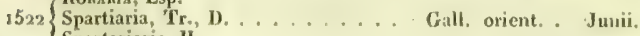

Spartariaria, H. 


\section{GENUS PHXLLOMETHA, Hambur}

Metamorphoses ignotx. - Antenure masis longiusculæ, pectinata , apice acutre. Palpi brevissimi. Lingua sub-nulla. - - Staturu debilior, sub-media. Alæ elongatulæ, cinerex, albo bistrigatæ, decussat $x$.

1523v. Gracilaria, Ramb, $F$, And, . . . Hispan. mer...

\section{GENUS ANISOPTERYX, Steph. Fidonia, Tr. Mibernia, Dup.}

Jarva Lavis, modice elongata, 12-podi, arboricola. Metamorphosis hypogxa. - Antennx maris pectinatx. Palpi breves. Lingua sub-rtulla. Thorax debilis, villosus. Abdomen longius. $\Lambda$ læ integerrima; antica lineis duabus sinuatis lunulaque media signata. Femina onmino aptera, araneiformis. - Alac maris decussatx ut apud Lithosias.

1524 Escularia, W. V., H., Tr., D. . . P., cte. .... Martis.

Murinaria, Esp.

27. GENUS CHEMERINA, Boisd.

Ligia, Rambur.

Larva elongata, lavis, Io-poda. Metamorphosis hypogxa. - Antenna maris longiores, anguste pectinatx. I'rons sub-cucullata. Lingua producta. Palpi brevissini. Corpus gracile. Alæ angustiores, decussatz. Alæ feminx abortive, volatu haud proprix.

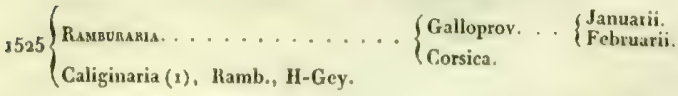

(1) Detecla ango $\mathbf{1 8 2 0}$ a Doin. Donsel circa Massiliam, et anno 1880 ir Corsica a Dom. Hambur. - Doni Dardoujo larvan teperil anno 1838 et jant specimina illasa in plarisque musxis Gallixe hospitantur.

Apud D. Treitschke species Cialiginata prius nominatur, quare noinen tuutsvi D. Mambur roultis novis speciebus hoe opusculum ausit; nomen illius hic insecto transferendo, singularem abservantiuin in illum derounsirare volui. 


\section{GENUS HIBERNIA, Latr., Dup. \\ Fidonice, 'Tr.}

Larvx laves, sub-elongatæ, 10-podx, arboricolx. Puppa sub-folliculata, vel intra quisquilias sepulta. - $\Lambda$ ntennæ maris pectinatæ. Palpi brevissimi. Lingua abortiva. Pedes elongati. Thorax mediocris, villosus. Alx integerrimx, sub-patulx; anticæ lineis duabus sinuatis lunulaque media signatæ. - Feminæ omnino apteræ, vel tantum rudimentis alarum præditæ. - Insceta hyemalia vel prodromaria.

$$
+ \text { Alæ glabrx. }
$$

1526 \{ceraria, W. V., H., Tr., D. .... Gallia, etc... Novemb. 1527 Rupicaprana, W. V., H., Tr., D. . Gallia, etc. . Novemb.

${ }_{1528}$ Aurantiarı, Esp., H., Tr., D. . . . Gallia, etc. . Novemb.

$1529\left\{\begin{array}{l}\text { Progemmaria, H., Tr., D. . . . . Gallia, etc. . } \\ \text { Capreolaria, Esp. }\end{array}\right.$

1530 Defoliana, L., H., etc. ....... Gallia, etc. . Novemb. 153 I $\left\{\begin{array}{l}\text { Leucoplleara, } \\ \text { Var. Nigricaria, H. H. II. Tr., D. G Gallia, etc. . Februarii. }\end{array}\right.$

${ }_{1532}\left\{\begin{array}{l}\text { Basaria, H., Tr., D. . . . . . . Galliabor.,etc. Novemb. } \\ \text { Erugaria, W. V., Esp. } \\ \text { Var. P Sordidaria, H. }\end{array}\right.$

t+ Alæ villosulæ. G. Phigalia, Dup.

${ }_{1533}\left\{\begin{array}{l}\text { Pitosaria, W, V., II., Tr., D. . ... E Europ. bor. . Februarii. } \\ \text { Plumaria, Esp. }\end{array}\right.$

Pedaria, Bork., F

29. GENUS NYSSIA, Dup.

Amphidasis, Tr.

Larvæ elongatæ, sub-lxves, vel tuberculis piliferis minimis praditx, metamorphosis hypogxa. Habitus Bombycum. - Antennæ maris pectinatz. Palpi breves, villosi. Lingua sub-nulla. Thorax robustissimus, lanatus. Abdomen crassum, conicum. Alæ robustæ. - Feminæ apterx. 
1534 Hispidarta, W. V., F., H., Tr., D. . . P., ete..., Martic

3535 Alminaria, Bork., H., Tr., D.... $\begin{aligned} & \text { Dalmatia.... } \\ & \text { Alp. Melr. }\end{aligned}$

1536r.Bomercaria (t)........... Valesia

Lombardia.

1537 Zonarta, W. V., H., Tr., D. ..... P., etc..... Aprilis.

1538 v.Gracarta.................... Foræa.... Februarii.

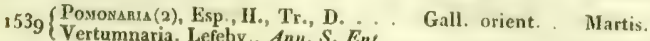

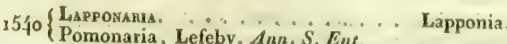

154. Pravaria, H. (an hujus gen.P). . . . L Lapponia.

\section{3o. GENUS AMPHIDASIS, Dup. Amphidasis, Tr. Biston, Iseach.}

Larvæ arboricolæ, elongatæ, tenue nodulosæ. Capite supra emarginato. Metamorphosis hypogxa. Habitus Bombycum, - Antennæ maris pectinatæ. Thorax validus, robustus, lanatus. Palpi breves, hirsuti. Lingua spuria. Caput retractum. Abdomen crassum, conicum: Alæ robustæ. - Feminæ alatæ.

+ Antennx cuspidatx.

- Alx fasciatr.

${ }_{15 / 2}\left\{\begin{array}{l}\text { Hirtakia, L., F., H., etc. ...... P. frequens... Martis. } \\ \text { Var.P Congencratia, H. }\end{array}\right.$

(1) Paulo major quam Zonaria et plane distincta ab umnibus specielus. Specimin. lustrata Ifirtariam adequant. Alo albida fasciis quinque nigro-fuscis. Thoras pilis albi indutus. Abdomen cinereo-testacenm. - In Valesia Dom. Anderregs, in Lumberdi. D. Villa invenerunt.

(2) Forsan aliquis mirabitur, nomen hujus speciei mutatum ot ad specirin sequentem, ut genuinan a Linzeo deseriptam, a D. $\boldsymbol{\Lambda}$. Lefebrre, suadente, ut monet, C. Dupoochel, translatum fuisse I

Apud Linnacum nulls hujus nomiois. species Nomen Pomonarie ab llubner illi Gen. netres es Europa centrali inditum fuit, et a DD. Borkhausen, Eisper et Treilschke usurpatum ad eamdem speciem designandam.

Cocterum de Lapponaria, g̨uamvis primo intuitu a Pomonaria nostra valde diversa, aherrationem hyperborean suspicari licet. 
* Alx irroratie.

8543 Beturaria, L., H., etc. . . ...... P., etc. .... Maii.

† Antennæ obtusæ.

1544 Prodromaria, F., W. V., H., Tr., D. P., etc. .... Martis.

\section{GenUS boARMia, Tr., Dup.}

Larva elongatæ, obscuræ, torulosæ vel ramiformes. Metamorphosis hypogæa vel intra quisquilias. - Antennæ maris pectinato-plumosæ, sæpius apice cuspidatæ. Palpi breves. Thorax robustior, squamosus. Abdomen longiusculum, sub-gracile. Alæ latæ, patulæ, concolores, fimbria sinuata; cinereo-nebulos a atomis fuscis irrorat $x$, lineis anguloso-nebulosis lunulaque vel puncto medio scriptx.

\section{$+$}

1545 Hontani, F., H., Tr., D. ...... An ex Amer. bor.?

1546 v.Gadithria (1)............. Hisp.australior.

$1547\left\{\begin{array}{l}\text { Repakdaria, W. V., H., Tr., D. ... Gall. bor. .. \{ Maii. } \\ \text { Var. Conversatia, H., D. }\end{array}\right.$

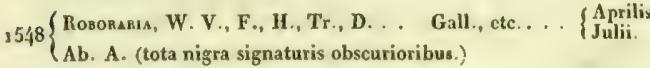

$\times 549\left\{\begin{array}{l}\text { Selenaria, W. V., H., Tr. . . . . } \\ \text { Consortaria, Bork. } \\ \text { Turcaria, F. }\end{array}\right.$

1550 Dsanakta, H. .......... Hungaria ,

1551 $\left\{\begin{array}{l}\text { Consortakia, F., Esp., Tr. . . . . G Gall., etc. . } \\ \text { Consobrinaria, H. }\end{array}\right.$

155. Ilicaria, H-Gey, . . . . . . . . Galloprovinc., Junii.

(1) Statura fore Hortaria. Facile distincte alin anbtus albis, unargine fusco limbsto. 


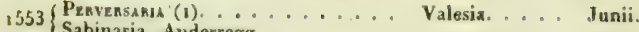

Sabinaria, Anderregg.

$1554\left\{\begin{array}{l}\text { Rnomboidakia, W. V., H., Tr., D. . Gallia, etc. . . } \\ \text { Gemmaria, Bork. }\end{array}\right.$

1555 Austensaria (2). . . . . . . . . Pyren..... Julii.

1556 Umaraka, H., D. . . . . . Monspelii. . A A pritis.

(Septembr.

1557 Ametaria, W. V., H., Tr., D. ...

${ }_{3} 558$ Secundani, W. V., H., Tr., D. ... \{ $\begin{gathered}\text { Pinetis Germa- } \\ \text { niæ..... Julii }\end{gathered}$

tt

$\$ 559$ Cinctania, W. V., Tr., D...... Gallia etc.... \{ Maii.

1560 Consumulakia, D.......... Gall mer.

1561 Occitanara, D. Frey. . . . . . . . Monspelii. . Septembr.

ttt

1562v, v. Souehana, Ramb-Ann, S. Ent... Galloprov.

1563 Sociaria (3), H., Tr., D. ....... Galli mer.... Septembr.

1564 Propisquaria (4)........... Monspelii.

3565 Lividani (5), H., Tr. . . . . . . Burgundia mer.

1566 Nrctemeraria (6), H., D. . . . . . Gall. mer.

(1) Atsais Rhonboidaria et forsan illius varietas Sabinivora. Signaturis vix differt a Ahomboidariis genuinis, at aspectu onnino siogulari alisque magis rotundatis facile distinguitur.

(2) Aftinis Rhomboidarice el forte illius aberratio Pyrenaica. Ala albide sigaaturis fere ut apud hhomboidariam, at magis macilentis.

(3) Aberrationem communicavit D. Baube cigereo-putverulentam, fasciis Iransversis omnino destitutam, sed late vigro limbatam.

(b) Aftiois Sociaria, at plane tistincta alis haud nebutoso-pulverulentis signaturisque diversis, magis ohscuriotibus.

(B) Larva cinerco-ocloulosa, collari aurantiaco pradita, ut apud Lasiocampas, prunum sjoosam depascit. - Delecta in Burgundia meridionali a Cl. Alartin.

(A) Miros poritissimum Treitsche hane speciem ad Pefrifieariam relulisse! Verisunile est illi genuinam. Vyctemerarian videndi occasionem nondum olsvenissf. 


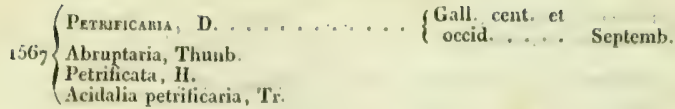
†t+† Larvæ lichenivoræ (an gen. prop.P)

${ }_{3} 568\left\{\begin{array}{l}\text { Geabmarı, H., Tr., D. . . . . . Gall. or. . . Julii. } \\ \text { Teneraria, H. }\end{array}\right.$

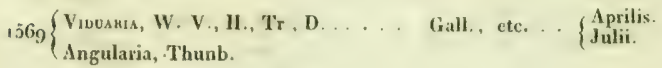

357o Lichenearia, W. V; H., Tr., D. . . . Gall., etc. . Julii.

\section{GENUS TEPHROSIA. Boarmia, Tr., Dup., Steph.}

Larvx elongat $x$, arboricol $x$, antice attenuat $x$, sub-tuberculat $x$; capite depresso, supra emarginato. Metamorphosis sub-folliculata, - $\mathbf{A} \mathbf{n}$ tennæ maris vix ciliatæ, sub-filiformes. Palpi squamosi, brevinsculi. Thorax squamulosus, debilior. Abdomen elongatum. Alæ patulæ, concolores, fusco-nebulose atomis lineisque sinuatis obscurioribus. Volatus sub-diurnus.

$$
\text { t }
$$

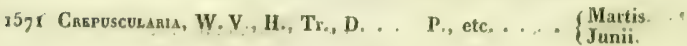

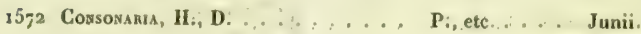
${ }_{1573}$ Extersakia, H., Tr., D. ...... $\begin{aligned} & \text { P., etc. . . } \\ & \text { Gall, bor. }\end{aligned}$

\section{$+t$}

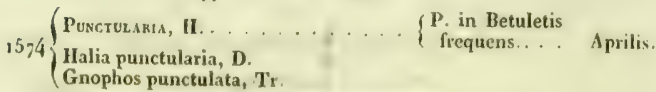

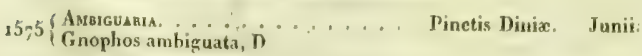




\section{3: GLNUS ELOPHOS. \\ Gnophos, Tr., Dup.}

Larvx ignotx. - Antennx elongatx, maris anguste pectinata. Palpi breves. Lingua distincta. Corpus debile. Alæ patulæ, debiliores, concolores, rotundatæ, sub-integerrimæ, ocello medio signat $x$; antic $x$ strigis duabus, posticæ unica. præditæ. Statura sat magna -Patria ad Alpes. - Volatus crepuscularis.

Hoc genus a genere Gnophos distinguitur alis integerrimis, antennisque pectinatis.

1576 Sordaria, Thunb. . . . . . . Dalecarlia. . Julii.

1577 Serotinama, IV. V., H., Tr., D. . . Diniz.: . . J Julii.:

15-8 Dilvcidaria, W, V., H., Tr, D. ..... Alp. callid. . Juhii.

1579v. Unicoloraria, Ramb: F. And. . . . Hispan, mer.

1580 "Zelleraha, Fr. . . . . . . . Alp. Bavar... Julii.

1581 Operaria, H., D. . . . . . . . . Mont. Occitan. Junii.

tt

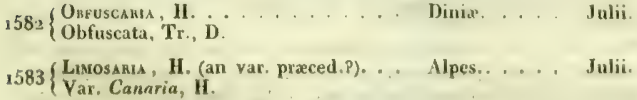

\section{GENUS GNOPHOS.}

Guophoses, Tr., Dup.

Larva rigidissima, breviusculæ, plantas hiumiles pascentes. - Antemux simplices in utroque sexu. Palpi brevissimi. Lingua exserta. Ala patulæ, concolores, ut apud Bonrmias, stigmate orbiculari signatæ. fuscr, atomis obscurioribus conspersæ; antica serratim bistrigat $x$; posticx profunde sinuato-fimbriatæ. Statura magna vel media.

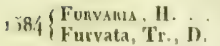

Alprs....... Iunit. 
$1585 \cdot\left\{\begin{array}{l}\text { Dugerana } \\ \text { Dumetata, } \mathrm{T}_{\mathrm{i}}\end{array}\right.$........ Ddhatia.

$2586\left\{\begin{array}{l}\text { Sartaria . . . . . . . . } \\ \text { Sartata, Tr. b., H-G. }\end{array}\right.$

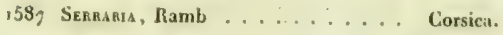

$1588\left\{\begin{array}{l}\text { Pullama, H. . . . . . . . Gall. mer. . Junii. } \\ \text { Pullata, Tr. D. }\end{array}\right.$

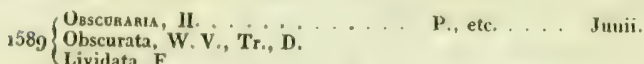

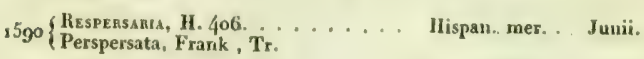

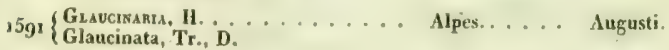

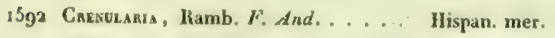

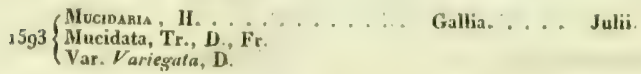

35. GENUS MNIOPHILA.

Hoarmia, Tr., Dup.

Jarva abbreviatx, depresse, tuberculis minimis praditx, lichenes murorum, lignique putrescentis pascentes. Metanorphosis snb-follicu. lata. - Antennæ maris pectinatx. Palpi brevissimi. Lingua vix ulla. Alæ tectiformes, breves, angustiores, discolores; anticæ cine. rex atomis lineisque duabus signata, Corpus minus debile. Statura parva.

s5gi Cinerania, F., W. V., H., Tr., H. . Gallia, mer. Julii

3595 CoRmicakia, H., D. ......... r. frequens, , Junii. 
36. GENUS BOLETOBIA.

Fidonia, Steph: Gnophos, Tr., D.

Larva abbreviata, tuberculis piliferis instructa, Boletis ligni putridi vescens. Metamorphosis sub quisquiliis. $-\Lambda$ ntennæx maris pectinata. Palpi graciles, valde elongati. Lingua producta. $\mathbf{A l x}$ concolores, patulæ. Corpus debile. Statura parva.

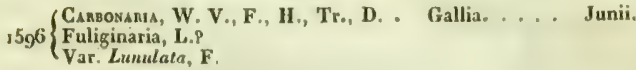

\section{GENUS EUBOLIA.}

Eubolice et Phasiance, Dup. Aspilates, Idea, Cidaria et Larentia, Tr

Larvæ læves, elongatx, capite rotundato, - Antennæ maris pectinatx. Palpi nudiusculi, acuti, ultra clypeum sxpius assurgentes. Alx integrx, cincrex; antic $x$ obscurius fasciat $x$, postic $x$ pallidiores, obsolete fasciatx.

Hoc genus nimis artificiale, simul Fidonins, Aspilntes Cidhariasque, tangere videtur.

+ Alæ cinereæ, atomariæ, margine obscuriori. Fidonic, Tr.

${ }^{5} 597$ Assimilanta, Ramb. Ans. S. Ent. . Corsica. ... Junii.

$1598^{\circ}$ Tephrarla. ........... Patria ?

Monusasu, W. V., II, Tr.; D., . . Gall, etc. . Junii. $1599\left\{\begin{array}{l}\text { Myosaria, Esp. } \\ \text { Var. Cineraria, }\end{array}\right.$

IGon v. Aremacearia, W. V., II., Tr., D. . Gall. or. . . Junii.

t† Alæ cinerea, lineis duabus flavis.

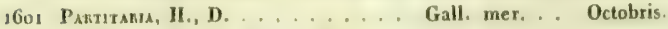

i6nz $\left\{\begin{array}{l}\text { Peltahia, Ramb. . . . . . . . Gall. mer. . Octobris. } \\ \text { Scutalaria, D }\end{array}\right.$

$\left\{\begin{array}{l}\text { Scutularia, D. } \\ \text { Permutaria, lirey. }\end{array}\right.$ 
tt+ Alæ cinerex, fascia niģa.

2603 Artestakis, WV. V., F., H., Tr. D. . Gall. mev. . Julii.

1 tiof Rippertana, D., H-Gey. . . . . . . Diniæ...... Julii.

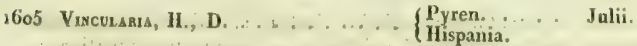

ftt $\mathrm{Alx}$ cinereæ vel fuscæ, striga apicali obliqua, fusca.

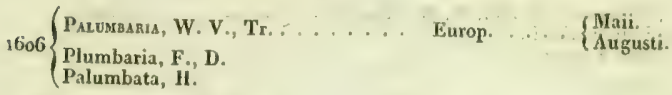

1607 Megsuramia, W. V., Tr., D. . . . . Europ. . . . Julii.

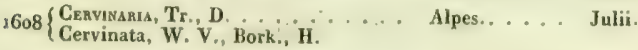

$1609\left\{\begin{array}{l}\text { Moemani, W: V., F., Tr., D. . . . Reg. alpin. ... Julii. } \\ \text { Moniata, H. }\end{array}\right.$

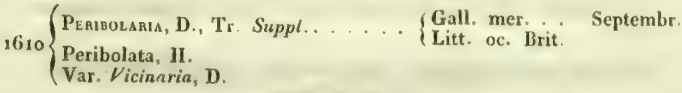

s6u Proxmmana, Ramb, Ann, S. Ent. . . Corsici...... Octobris.

$1612^{*}\left\{\begin{array}{l}\text { Duplicara. } \\ \text { Duplicata, H. Ho . . . . Italia. }\end{array}\right.$

$16,3\left\{\begin{array}{l}\text { Conssaria, } \\ \text { Duplicata, ii }\end{array}\right.$

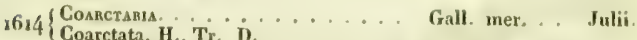

${ }_{1615^{\circ}}\left\{\begin{array}{l}\text { Simpliciaria } \\ \text { Simpliciata, } \\ \text { Tr. . . . . B . Batus }\end{array}\right.$

616 Bipunctakia, W. V. F., Bork., Tr., D. Europ. . . Julii.

$1617 \mid \begin{aligned} & \text { Nebularis. } \\ & \text { Nebulata, Fr., Tr., H-Gey. . . . . G Galloprov. }\end{aligned}$ 
$\mathrm{Ht+t}$ Alæ fasciato-nebulosæ.

$1618\left\{\begin{array}{l}\text { Vespentamia, } \\ \text { Vespertata, L., il, etc. }\end{array}\right.$

Alpes. ... . . Julii.

$1619^{\circ}\left\{\begin{array}{l}\text { Incursaris. } \\ \text { Incursata, H. }\end{array}\right.$

Alp. Hungar.

1620 Polygraphahu (1)......... Dalecarlia.

1621 Allemania (2), . . . ....... Lapponia.

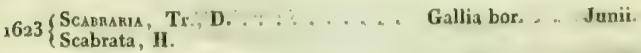

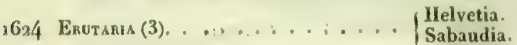

Alæ virescentes.

625 Lotakin $\ldots \ldots \ldots$ Gall, mer.

Aquaria, Tr., Fr.

${ }_{162} 6$ Amlutaria : . . . . . Gall. mer.

Olivaria, D.

${ }_{1627}\left\{\begin{array}{l}\text { Mrsma, W. V., Tr., D. . . . . . Europ. .... Junii. } \\ \text { Miata, H. }\end{array}\right.$

Viridaria, $\mathrm{F}$.

\section{$t+t+t$}

$1628\left\{\begin{array}{l}\text { Fernugaria, W. V., Bork, Tr., D. . Europ. . . . . Maik. } \\ \text { Quadrifasciata, H. }\end{array}\right.$

$2629\left\{\begin{array}{l}\text { Quadarasciara, W. V., F., Tr., D. Gallia. .... Julii. } \\ \text { Ferrugaria, H. }\end{array}\right.$

(1) Paulo major Salicara illiusque sub-affiois; olis anticis cinereo-allsidis afomis obscurioribus variegatis, puncto fasciisq̨ue tribus fuscis, exlerna angulosa ; linea fulgurali albida ut apud Molluginarian; posticis albidis puncto medio nigro margineque externo fuscescente. Feminam tantum vidi.

(a) Stutura Seabraria; alis albo cinereoque variis, fasciis quatuor fuscis, externa marginali linea fulgurali albida, divisa.

(a) Statura Vesperlario. Ala anticæ albida lineis transversis geminatis fuscis, fuscia lim rlegestis; fasciis binis exiernis nervis nigris srigulalis. Ala postica albidre ad apicem, apud frminam lineis cinercis undulate. 
1630 $\left\{\begin{array}{l}\text { Ligusinarı, Tr., D. . . . . . . Gallia. . . . } \\ \text { Ligustrata, H. }\end{array}\right.$

\section{GENUS ANAITIS, Dup. \\ Larentice et Aspilates, Tr.}

Larvæ læves, elongatæ, sub-depressæ, IIypericicolæ.-Antenna filiformes in utroque sexu. Palpi ultra clypeum assurgentes. Lingua producta. Alæe integræ, vix patul $x$, cinereæ lineis angulatis, numerosis, trifariis. -Volatus diurnus.

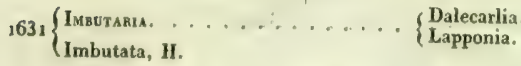

3632" $\left\{\begin{array}{l}\text { Sororaria (an vax. major præced.P). Helvetia. } \\ \text { Sororiata, H., Tr., D. }\end{array}\right.$

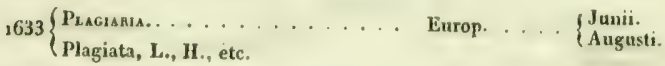

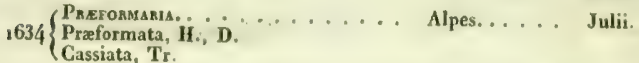

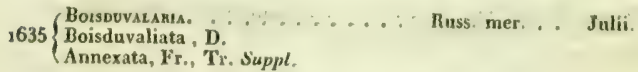

39. GENUS LARENTIA.

Larentia, Tr., D. et Acidalia; Tr.

Larvæ breviusculæ, capite parvo, convexo, læves. Metamorphosis hypogxa vel sub quisquiliis. - Antennæ simplices in utroque sexu. Palpi elongatuli, ultra clypeum assurgentes. $\Lambda$ lx integra, vel undulato-dentatæe, lineis transversis, numerosis, undulatis, parallelis, in anticis evidentioribus. Abdomen clongatum, apice apud mares sxpius valde penicillatum.

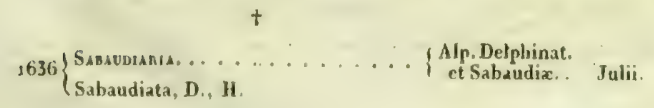




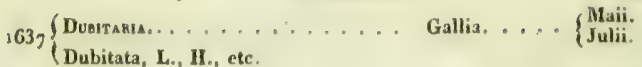

(Certania................. Mont. Gall. . Julii.

638 Certata, H., Vere., Tr., D.

Cervinata, H. 266 .

$1639\left\{\begin{array}{l}\text { MontryagnRz } \\ \text { Montivagata, } \\ \text { B., D. }\end{array}\right.$

Diniæ...... Julii.

1640 v. Nevadaria, Ramb. F. And.: . . : Sierra nevada.

$+t$

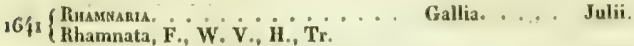

1642 Vetularia. ............... Junii.

${ }_{3} 643$ \{ Undurana. U.......... Gall. bor. . Junii.

$164^{3}$ Undulata, L., H, etc.

tt+

,644\{ Gemarana.............. Gall. mex.

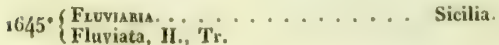

$+1+t$

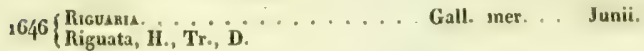

1647 Bilinearla. . . . . . . . E Europ.

$1648 \cdot\left\{\begin{array}{l}\text { Bistrigaria }(1) \ldots \ldots \ldots \\ \text { Bistrigata, Tr. }\end{array}\right.$

\section{$\mathrm{HHt+}$}

$1649^{\circ}$ Alvtacearia. .............. Dalmatia.

Collarıa . H. ......... Gall. mer. . Aprilis.

1650 Collata, H., Tr., D.

Var. Asperarin, H.
Var. Pilyats, Ramb.

(1) Specinins Corsica, sepius colore Bilinearia australis, at semper facile distincto liaea angulosa paginse inferioris. 


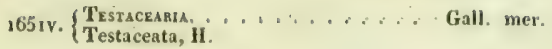

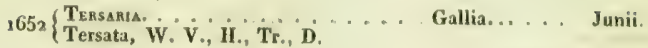

$1653^{\circ}\left\{\begin{array}{l}\text { Emolakia (an var. praced. P) .... Patria ? } \\ \text { Emulata, H. }\end{array}\right.$

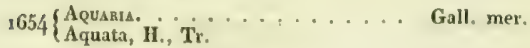

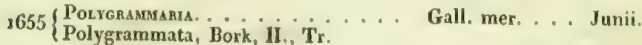

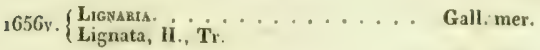

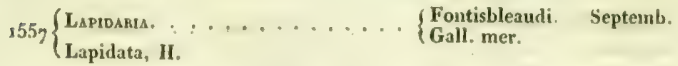

$t+t+t$

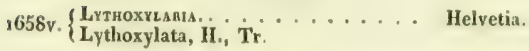

$1659\left\{\begin{array}{l}\text { Petraria, Esp., H., Tr., D. . . . P. . . . . J Junii. } \\ \text { Virgaria, Bork. }\end{array}\right.$

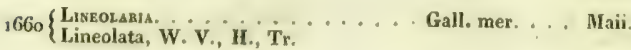

\section{$t+t+t+$}

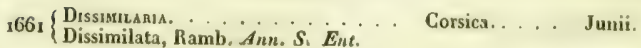

${ }_{1662}\left\{\begin{array}{l}\text { Scaptorsais, W. V. ........ Gallia...... Julii. } \\ \text { Scripturata, II., Tr., D. }\end{array}\right.$

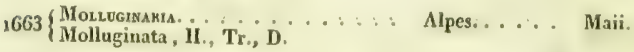

\section{ttttttt}

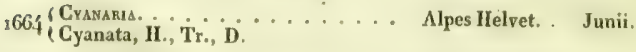

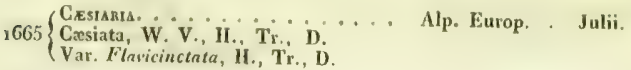


$1666\left\{\begin{array}{l}\text { Porania, } \\ \text { Polata, B, olim, D. }\end{array}\right.$

Lapponia.... Augusti.

$1667\left\{\begin{array}{l}\text { Psittacaria. . . . . . . } \\ \text { P'sittacata, F., II., Tr., D. }\end{array}\right.$

Europ. .... $\left\{\begin{array}{l}\text { Maii. } \\ \text { Septembr. }\end{array}\right.$

1668 Cонасияи (an yar, præcedent, p).... Europ. bor. . . Septembr. Coraciata, H., Tr., D.

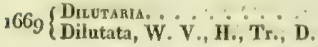

Europ. .... Octobr.

$1670\left\{\begin{array}{l}\text { Brumaras } \\ \text { Brumata }\end{array}\right.$

1678 Boreara . .............. Europ, bor. . Novemb.

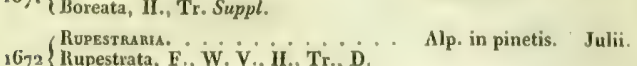

$1672\left\{\begin{array}{l}\text { liupestrata, E, W. V., H., Tr. D. } \\ \text { Var: Q Bassiaria, Feisth. Ann. S. Ene. }\end{array}\right.$

40. GENUS LOBOPHORA, Curtis. Amathia, Dup. Acidalia, Tr.

Larvæ arboricolæ, læves, capite plano, postice emarginato; ano bifido. Metamorphosis hypogæa.-Antennæ simplices. Pal pi breviores. Lingua producta. Alæ integræ; anticæ lineis undulatis, parallelis, signatæ; posticx latiores, pallidæ, apud mares ad basin marginis interni lobulo membranaceo præditæ.

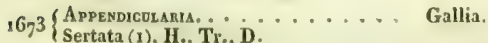

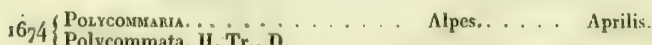

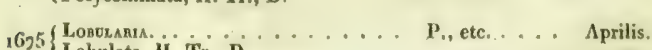

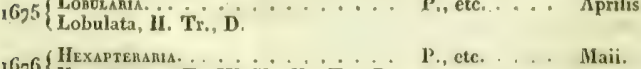

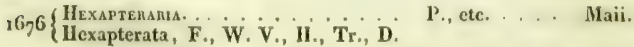

$16_{77}\left\{\begin{array}{l}\text { Sexaranu. . } \\ \text { Sexalata, Bork, Tr, } \\ \text { Sezalata, H. }\end{array}\right.$

(1) Nomen Sertate mutandum censui, utpote noinini Certata nimis simile. 


\section{GEIVUS EUPITHECIA, Curtis.}

Larentice, Tr., Dup.

Larvæ læves, cylindric $x$, modice clongatæ, capite minuto, plane globuloso. Metamorplosis folliculata vel sub quisquiliis, - Antennæ in utroque sexu simplices. Palpi elongati, ultra clypeum sub-assurgentes. - Insecta minuta; alis anticis angustioribus, sxpius clongatolanceolatis, lineis transversis undatis numerosis.

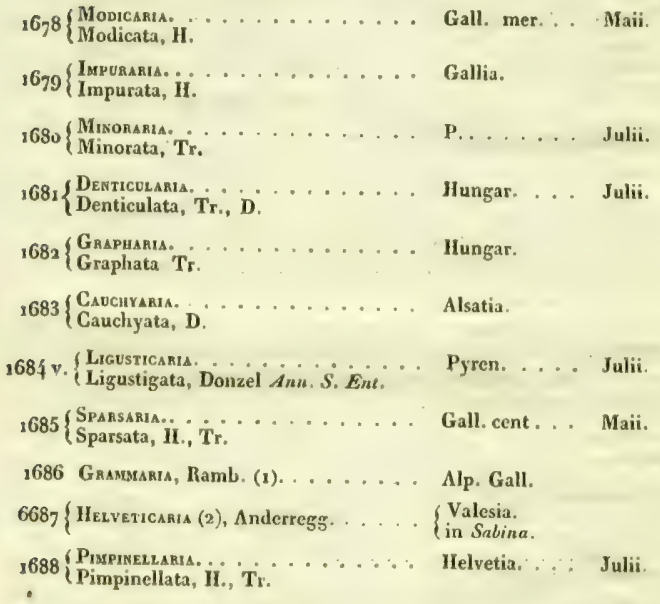

(1) Statura et sumina atinitas //veticariut at distiacta. Also anticac pallidiores strigis undulatis nervisque interruptis cinereo-albidis; puncto discoidali nigro crassiori. Ala postica ad apicem obscuriores cinereo-undulata. In abicte alpium circa la grande Cherleuse, larvam invenit Doctor hambur.

(2) Valde aftanis Pimpinellaria ab llubnero depictre. Alae antica pallide fusca strigis obsoletis pollidioribus, nervis fuscis cinereo strigulatis; puocto discoidali nigro, crassiori. Ala postice cinereo-fuacae. - Io Iunipero Sabina larvam invenit D. Anderres5 circa Gamsem in Valesia. 
1689 Satragra ............ Gall. $\mathrm{Al}$.

(Satyrata, II., Tr.

$16 g \circ$ Residuara. ........... Dalmatia

s691 Recictaria (1)........... P....... Junii.

$169^{2}\left\{\begin{array}{l}\text { Oxydara, } \\ \text { Oxydata, Tr. } \\ \text { Distati. }\end{array}\right.$

Disparata, H. 247 .

Succenturaria. ............ Gallia...... Julii.

Disparata, H. 246.

$1694\left\{\begin{array}{l}\text { Centaurearia. . . . . . . . Gall., etc... } \\ \text { Centaureata, H., Tr., D. }\end{array}\right.$

$1695\left\{\begin{array}{l}\text { Extremak1 .............. } \\ \text { Extremata, H. }\end{array}\right.$

$1696\left\{\begin{array}{l}\text { Irrigdana } \\ \text { Irriguata, H. }\end{array} \mathrm{Tr}_{\mathrm{r}} \ldots . . . . .\right.$. Gall. mer. . . Junii

$1697\{$ Exıguaria............... Gall ia. . . Julii.

1698 Reductaria (2) . . . . . . . . P. frequens.

$1699\{$ InnotaRı . . . . . . . . . P. . . ... Maii.

1700 Lanceolakin, Ramb. $F$. And...... Hispan. mer.

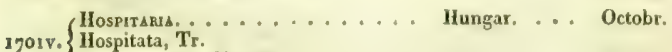

folv. $\left\{\begin{array}{l}\text { Hospitata, Tr. } \\ \text { Succenturiata, H. } 236 .\end{array}\right.$

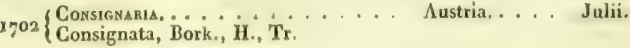

(1) Statura Centaurearia; inter omnes facile distincta. Alse antica albido-fusca, ad basin fascia lateritia, in medio fascia lata fusca signate; ad apicem albido-rubrotinctre, fusco jnquinate. Postice fere uti antica depicte. Femina major, pallidior fasciis rubris evidentioribus. - Circa Parisios roinus frequens.

(2) Statura et affinitas Exiguaria et in multis Musæis verisimile cum Exigunria genuina confusa. Alve antico etongata, cinereo-fuscestentes fasciis obscurioribus lineacue terminali pallida; ante apicem strigulis geminatis tongitudinalibus nigris. Alat postic pallidiores - - Cirea Parisios frequens mease Maio. 
$1703\left\{\begin{array}{l}\text { Verosaria, } \\ \text { Venosata, H }\end{array}\right.$

170 Ul Utimaria (1), Ramb. ......... Galloprov.

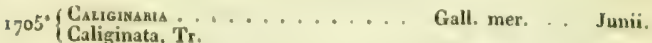

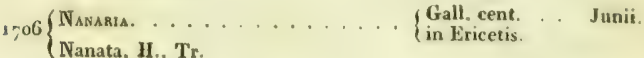

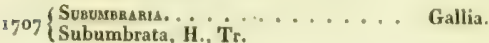

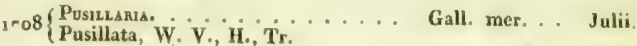

1709 Tantulataia(2), Ramb. . . . . . F Gall. mer.

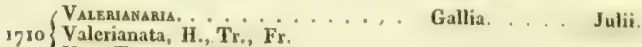

(Var, Tenuiata, H.

1711 v.Pauxizlakta (3), Kamb. . . . . . Gall. met.

$1712^{\circ}\left\{\begin{array}{l}\text { TaAsarsciaria............ Bavaria. } \\ \text { Tamarisciata, Fr. }\end{array}\right.$

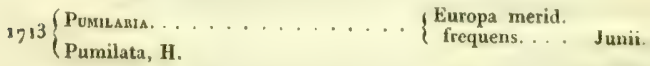

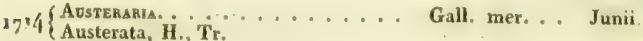

715 v. $\left\{\begin{array}{l}\text { Castigaria } \\ \text { Castigata, H. . . . . . . . Gall, mer. }\end{array}\right.$

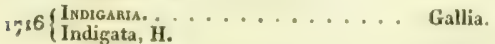

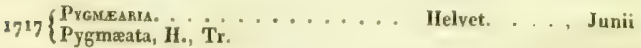

(1) Minimis minor. Alæo omnes cinerex, obscurjus limbato punctoque discoidali nigro; anticae elongate, punctulis Tribus costalibus, nigris, obsoletis, signate. - Circa Massiliam invenerunt DD. Rambur et Dardouin.

(2) Valde allinis Pusillaria at minor. Alse antice cioereo-fuscxe, slbido immista, puncto discoidali nigro fasciisque quatuor undulatis fuscis ; postica pallida puncto discoidali lineisque undulatis ad apieem fuscis. Differt a Pusillaria proter staturam miaorem lineis magis regularibus obscurioribus.

(8) Staturs fere Sobrinaria. Also cinereo-pallida, pulverulenta; antice isnceolats, fasciis angulato-flexis puncloque discoidali signata; postica uniculores, immaculatr, -In Gallia australiori detecta $\approx \mathrm{Cl}$. Cantener. 
m18 Minutata, H., Tr., Frey.

Gallia. . : . Junii.

Var. Alsynthiata, $\mathrm{H}$.

$1719\left\{\begin{array}{l}\text { Denotaria. } \\ \text { Denotata, H. } \\ \text { Scabiosata, D. }\end{array}\right.$

Fall. bor.

Scabiosata, D., Bork.?

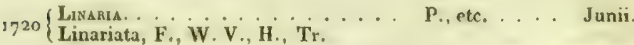

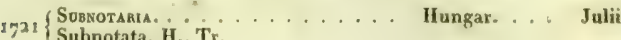

${ }_{1722}\{$ Togaria. . . . . . . Gall. mer.

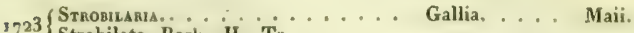

Rectanguraria. .................. Julii.

172 t $\left\{\begin{array}{l}\text { Rectangulata, F, iI., Tr. } \\ \text { Var. Coronala, H. }\end{array}\right.$

$2725^{\circ}\left\{\begin{array}{l}\text { Suвқranis. } \\ \text { Subærata, iI. }\end{array}\right.$

Patria ?

$1726\left\{\begin{array}{l}\text { Debilarua. } \\ \text { Debiliata, H. }\end{array}\right.$

German. ... Junii.

1727 Begrundanta $(\mathrm{r}), \ldots \ldots \ldots \ldots$

P. . . . Junii,

1728 Isogramararia. . . . . . . . Hungar. . . Junii,

$\$ 729$ \{rdoniaria ................ Julii.

Intorbanis. ................. Austria... Junii.

$1730\{$ Inturbata, H., Tr, Fr.

173i Ebicearia, Ramb. Ann. S. Ent.... Gall. mer.

1732 Gunardurta (2)......... $\begin{aligned} & \text { Monspelii. } \\ & \text { Corsica. }\end{aligned}$

(1) Statura Debiliaria at paulo minor alisque magis roluadatis. Alwo omnes pallide cinerea, ad apicem ohscuriores; lineis plurimis confusis, undulato-regularibus, pallidis, fere albidis, penultima latiori, geminata, evidentiori. Alos antica puneto centrali sub-destitutee, - Circa Parisios mense Junio iavenit D. Begrand.

(2) Statura Scoparia, illiusque affiais. Alw antice fusco-rủbricantes, nervis nigris ulbido strigulatis lineisque plurimis uadulatis albis; in medio linea albida geminata valde coospicua ut apud Scopariam. Postico fusco-rubricantes lineis transversis undulatis obseurioribus -D. Guinard Entomologo Monspelieusi dicala. 


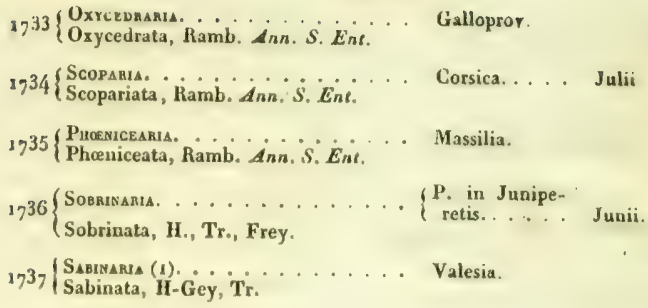

\section{GENUS CHESIAS.}

Chesias, Tr., Dup.

Larve lreves, elongatulx, capite globuloso. Metamorphosis hypogres. - Antennæe elongatæ, filiformes. Palpi ultra clypeum assurgentes. Lingua breviuscula. Alx per quietem acute tectiformes; anticre elongato-lanceolatæ; posticæ rotundatæ.

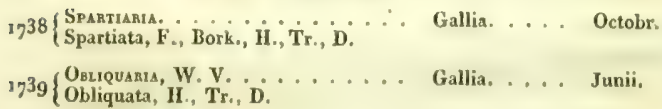

43. GENUS CIDARIA, Tr., Dup.

Larva læves, minus elongatæ. Metamorphosis hypogæa. - Antenne simplices. Palpi ultra clypeum assurgentes. Alæ latiusculæ; anticæ marmoratx, fascia lata, extus angulosa, depictx. - Volatus crepuscularis.

$+$ $1740\left\{\begin{array}{l}\text { Sagitrakia } \\ \text { Sagittata, F, Tr., D. } \\ \text { Comitata, H. }\end{array}\right.$

(1) In genere gigsotes. 


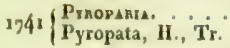

Russ. mar.

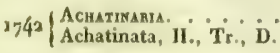

Gall. bor... J Julii.

${ }^{3} 74^{3}\left\{\begin{array}{l}\text { Popularia. } \\ \text { Populata, L., etc. }\end{array}\right.$

Europ. . ... Junii.

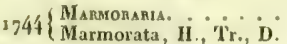

Gall, mer. .. Junii.

$1745\left\{\begin{array}{l}\text { Pyraliarsa } \\ \text { Pyraliata, W. V. F. H., Tre, i. }\end{array}\right.$

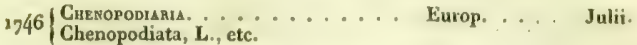

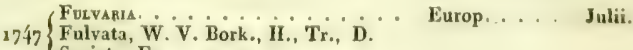

+† Chesias, Tr., Dup.

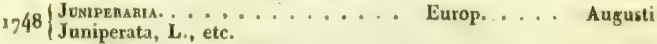

$1749 \begin{aligned} & \text { Varuarla. } \\ & \text { Variata, W. V., H., Tr., D. } \\ & \text { Var. Obeliscata, H., Tr., D. } \\ & \text { Var. Vitiosata, Fr. }\end{aligned}$

$1750\left\{\begin{array}{l}\text { Ruptaria. } \\ \text { Ruptata, H., Tr. D. . . . . . Gall. cent. . Junii. }\end{array}\right.$

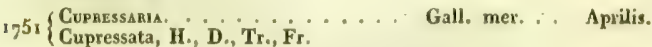

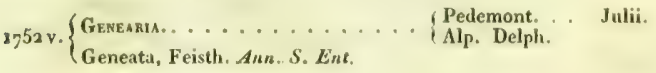

1753 Ulicara Ramb. Ann. S. Ent.... Gallopror . Junii.

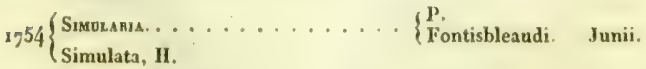

1755 Frabrana, 1., Tr. . . . . . . . Silesia. . . Augusti.

$t+t$

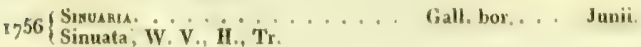




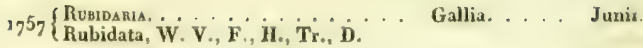

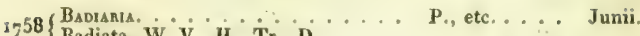

${ }-59\left\{\begin{array}{l}\text { Berderaria. . . . . . . . P., etc. . . . } \\ \text { Berberata, W. V., F., H. Tr, D. }\end{array}\right.$

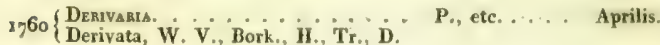

$+t+t+$

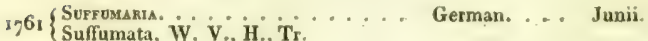

1762 \{ressana. . . . . . . Europ. . . . Julii.

I7 63 Sll sceara. ............ Gall. bor. . Junii.

j64v. Reticulara.................. Justria.... Julii.

Rossaria. ............ Gallia, ctc... Junii.

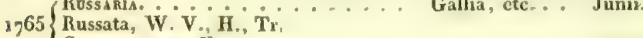

Centumnotata, $\mathrm{F}$.

\section{ttttt}

${ }_{7} 66\left\{\begin{array}{l}\text { Erdraku, } \\ \text { Elutata, H. Tr. }\end{array}\right.$...... Gall, bor, etc. Juni.

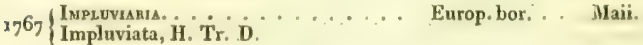

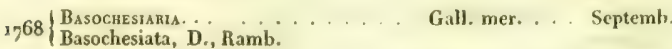

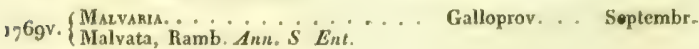

$+t+t+t$

${ }_{1770}\left\{\begin{array}{l}\text { Munitana } \\ \text { Munitata , H. . . . . . . Suecia. }\end{array}\right.$

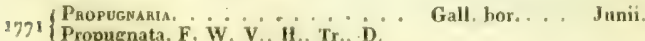

(1) Nonen Prunaria alii speciei jam inditum. 


\section{thttttt}

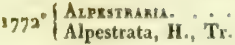

Helvet. ... Julii.

${ }_{1773}\left\{\begin{array}{l}\text { VRREтARа } \\ \text { Viretata, H., Tr., D. }\end{array}\right.$

P. ..... Junii.

$1774\left\{\begin{array}{l}\text { Frustrana. Frustrata, Tr., Frey. } \\ \text { Muscosata, }\end{array}\right.$

Diniæ.

Muscosata, Donzel. Ann. S, Ent.

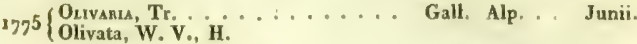

$3776 \mathrm{v} .\left\{\begin{array}{l}\text { Tophaceara } \\ \text { Tophaceata, W., V., i., Tr. }\end{array}\right.$

Alp. Helvet. . Julii.

$1777\left\{\begin{array}{l}\text { Picaras } \\ \text { Picata, H., D., Tr. }\end{array}\right.$

P.

$1778\left\{\begin{array}{l}\text { Aptania, } \\ \text { Aptata, H., Tr. }\end{array}\right.$

Alpes. ...., Julii.

\section{GENUS MELANIPPE, Dup. \\ Acidalize, Cidaria et Zerenes, Tr.}

Larvæ læves, elongatæ, strigatæ, capite rotundato. Metamorphosis sub-folliculata. - - ntennæ simplices. Palpi breves, acnti. Lingua brevis. Alx integræ, fascia marginali sub-interrupta terminatx, nigro alboque rivulato-maculatæ.

+ Alæ flavæ. G. Venilia, D.

$1779\left\{\begin{array}{l}\text { Maculanıa, L., H. } \\ \text { Maculata, F., W. V., Tr., D. . . Europ. . . . Maii. }\end{array}\right.$

$+\dagger$

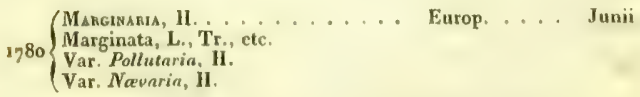

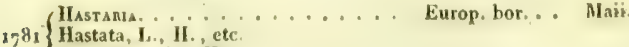

Var. Hastulata, H. 


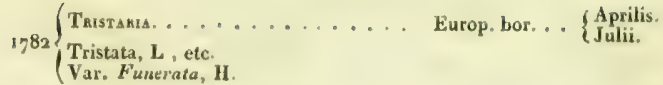

$\operatorname{s} 83\left\{\begin{array}{l}\text { Loctuara } \\ \text { Luctuata, W. }\end{array}\right.$

1784 \{ $\left\{\begin{array}{l}\text { Turbara, Tr. } \\ \text { Turbata, H. D. }\end{array}\right.$

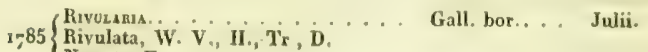

1786 v. $\left\{\begin{array}{l}\text { Hrdrara. } \\ \text { Ilydrata, Fr., Tr. Suppl. . . . Germania. Julii. }\end{array}\right.$

278 \{ Rivaria................. Gallia, etc. Julii,

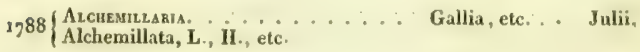

${ }_{72} 89$ v. $\left\{\begin{array}{l}\text { Amricularia. . . . . . . . . Gall. mer. } \\ \text { Amniculatz, H. }\end{array}\right.$
Gall. bor... Julii.

Alp. Helv. . J Julii.

\section{GENUS MELANTHLA.}

Zerenes, Acidalia et Cidarix, Tr.

Larva laves, elongatula, capite depresso. Metamorphosis sub-folliculata. - Antenna simplices, rarius crenulatxe. Palpi brevissimi. Lingua producta. Ala integræ, albx; anticx basi infuscatæ, medio (rarius ad marginem) fascia fusca signatæ.

† Fascia media integra.

$2790\left\{\begin{array}{l}\text { Montanaria, Tr., D. . . . . . P., ctc. ... } \\ \text { Montanata, W. V., H. }\end{array}\right.$

2794 Feisthamerciria (1).......... Sardinia.

(1) Slatura Flucturria affinitas autem Monlanaric. Ale solice albo, babi, fascia suedia, maculaque ante apicem futcis; punclis minimis margioalibus punctoque discoifuli aigrus. Ala postica atbirfe puneilis aigris marginalibus. - In Musco D. Feisthamat bospitatur. 


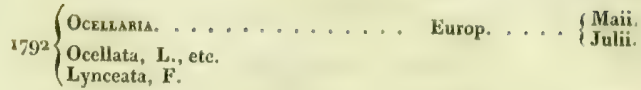

${ }^{3} 79^{3}\left\{\begin{array}{l}\text { Fluctuaria } \\ \text { Fluctuata, L., ete. . . . . . E Europ. . . Junii. }\end{array}\right.$

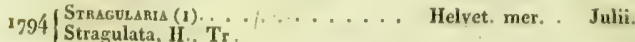

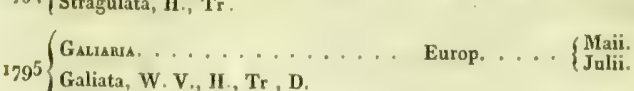
Var. Chalybeata, H.

†† Fascia media interrupta.

$1796\left\{\begin{array}{l}\text { Blakdaria, } \\ \text { Blandiata, W, V., H., Tr., D. }\end{array}\right.$

1797 Jucundabia (2). . . . . . . . Helvetia. . . Junii.

${ }^{8798}$ Scitexaria, Ramb. Ann. S. Ent. . . Gall. mer.

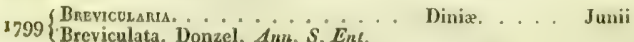

$1800\{$ Rubigrnaria. . ................... Punii.

Rubiginata, W. vi, F., $\dot{H}$., $\dot{T}_{\text {r., }}$ i.

180 s Procerlarka, .......... Junii

Procellata, W. v.. il., Tr.,

t†t Fascia media nulla.

$1802\left\{\begin{array}{l}\text { Adustania. . . . . . . . . P., etc. . . . I Junii. } \\ \text { Adustati, W. V., F., Tr., H., D. }\end{array}\right.$

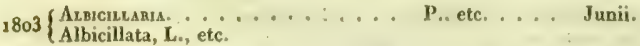

(1) Cl. Treitschke in supplemento Stragulatam ut Varietaten ad Fluetuaviam inmerito vefert. Stragulata vera IIubneri omnino diversa et vir ejusdem Generis! forte varietatis Fluctuarie ante oculos habuerit.

(g) Statura Blandiaria et forane tantum varietas alpiua. Ala abtica carneo-albida, basi, fascia nedia extus angulosa, puncto discoidali nigro signata, fasciaque marginali line albida divisa, fuscis. Als postico albida. - In alpibus Sabaudio inventa a Cl. D. Pierret, publicorum expensorum probatore aupremo necnon Numismatugraphe insigni. 


\author{
46. GENUS ZERENE, Dup. \\ Zerenes, Tr. Xerene, Steph.
}

Larvæ modice elongatæ, læves, passim pilosulæ. Metamorphosis hypogæa, vel sub-folliculata. - Antennæ simplices. Palpi brevissimi. Lingua elongata. Alæ integra, omnes albidæ, pustulatæ, vel punctatæ. Abdomen sæius punctatum. - Volatus crepuscularis. Statura major.

+ Alx pustulate.

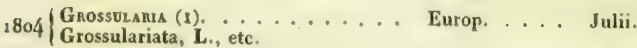

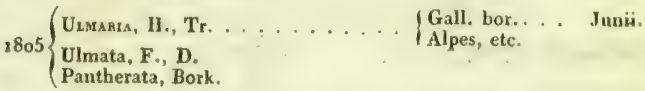

$1806\left\{\begin{array}{l}\text { Pantaria, L., H., Tr. . . . . . . Gall. mer. . Maii. } \\ \text { Pantata, D. }\end{array}\right.$

it Alæe punctatæ.

$1807\left\{\begin{array}{l}\text { Crtbanra. . . . . . . . . } \\ \text { Cribrata, H., Tr., D. }\end{array}\right.$

47. GENUS CABERA, Dup.

Cnberae et Fidonice, Tr.

Larvæ graciles, modice elongatæ, tenue verrucosæ. Metamorphosis subfolliculata. - Antennx maris pectinatx. Palpi breves, vix ultraclypeum assurgentes. Alæ tenues, albidæ, pulverulcntæ, lineis plurimis transversis, per quietem patula. - Volatus crepuscularis. Statura media vel majuscula.

+ Lineis obsoletissimis, G. Corycia, D.

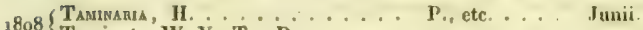

Taminata, W. V., Tr., D.

(1) Ne forte Grossulariaria, Linariaria, ste, scribere cogerer, repetitionem finis aria, eupbosia gratia, abstuli. 
t+ Alæ pulverulentz.

- Immaculatx.

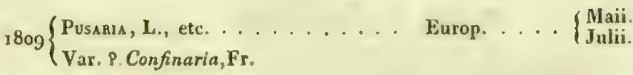

18 rov.Alezolakia, Ramb, $F$. And. . . . Hispan, mer.

Exanthemaria, Espe, Tro, D. . . . P. P., etc. .... Junii. I8 1 ₹ Exanthemata, W. V.

Striaria, H.

$1812\left\{\begin{array}{l}\text { Strigillaria, Esp., Tr., D. . . . Europ. . . . . } \\ \text { Respersaria, H. }\end{array}\right.$

$18 ı 3$ v.Graelsaria, Feisth. Ain. S. Eut, .. Catalaunia. . . Junii.

1814 Inquinatakia (1), Ramb. F. And. . Hispan. mer.

- Alæ contaminatæ.

1815 Contamumaria, H., D. ....... P...... Julii.

1816 Permutaria, II., D. . . . . . . Gall. mer. . . $\begin{aligned} & \text { Aprilis. } \\ & \text { Augusti. }\end{aligned}$

1817 Comsutara, H. (an var. praced,8). . Gallia mer. . Julii.

1818 Drekctarta. . . . . . . . . Hungar. . . Junii.

1819 Carana................. Gall. bor. . . Julii.

$+t+$

$1820\left\{\begin{array}{l}\text { Onowami, Borkl., H., Tr., D. . . G Gall. mer. . . Junii. } \\ \text { Var. Sudataria, H. } \\ \text { Var. Fecalaria, H. }\end{array}\right.$

(1) Altois Contaminaria at plabe distincta, defectu plago rubigineo-pulverulentre at marginem internum alarum anticarum, lineisque duabus omoino parallelis wque distautibus. 
48. GENUS EPHXRA, Dup.

Caberce, Tr.

Larvæ arboricolæ, laves, elongatulæ, lineatæ, capite depresso. Puppæe truncatæ, ano filoque transverso alligatæ. - Antennæ maris pectinatx vel dentatx. Palpi inclinati, mediocres. Lingua producta. Alæ integræ, patulæ, concolores, pulverulentæ, sæius signo hoc 0 medio notata, rarius tantum lineatx. - Volatus crepuscularis. Statura sub-media.

\section{t Alæ fuscæ.}

1821 Pictania (1), Curtis......... Gall. centr. . Aprilis.

t+ Ala lineatæ.

I822 $\left\{\begin{array}{l}\text { Thuliveanı, Bork., Tr., D. . . . } \\ \text { Linearia, H. }\end{array}\right.$

1823 Punctara, L, etc. ......... P., etc... Maii.

tt Alæ omicronatæ.

1824v. Pelvisaria, Ramb. $F$. And...... Hispan. mer.

$1825\left\{\begin{array}{l}\text { Porasta, Tr., D. . . . . . . G . Gall. mer. . Junii. } \\ \text { Porata, L.,F. F. V., II. } \\ \text { Punctaria, W. V. }\end{array}\right.$

$1826\left\{\begin{array}{l}\text { Pupıllania, 11., Tr., D. . . . . . Gall. mer.. . Septemhr. } \\ \text { Var. Nolaria, II. }\end{array}\right.$

1827 Grraria, Tr., D. . . . . . . . Monspelii.

$1828\left\{\begin{array}{l}\text { Argushria, } \\ \text { Ocellaria, H., Tr., D. . . . . . Iungar. }\end{array}\right.$

1829 Pendularta, L., etc. ........ P., etc. ... $\begin{aligned} & \text { Maii } \\ & \text { Augusti. }\end{aligned}$

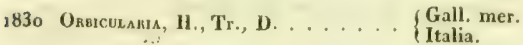

(1) Vix hujus seneris. Statura Caberas Pusaria; ala antica, pulverulento-fusca puncto magno discoidali, basi, lineis duobus, transwersis, sinualis, punclisegue inarginajibus fuscis. Postica cinereo-albida punclo centrali, linea serrata, sub-media, puactinque margiaalikus fuscis. - Circa Chälecu-da-Loir, e larva educta a Cl. A. Graslin. 
1831 Unicnomsra, W.V.,Esp,,H.,Tr., D. . P., etc. . . . \{ Maii.

\section{GENUS ACIDALIA.}

Dosythea et Acidalia, Dup. Idea et Acidalia, Tr.

Larvæ elongatæ, tenues, læves, plantis humilibus vescentes. Metamorphosis sub-folliculata vel hypogea. - Antennæ maris simplices, ciliatæ, vel tenue pectinatx. Palpi brevissimi. Lingua sub-producta. Alæ patulæ lineis angustis, transversis signatx. - Volatus subdiurnus. Statura parva, rarius sub-media.

+ Lineis mediis obliteratis, margine externo fascia macalari signato.

G. Dosychea, Dup.

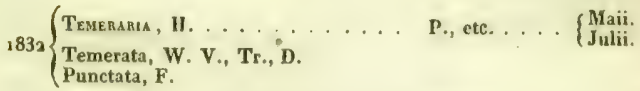

$1833^{\circ}\left\{\begin{array}{l}\text { Comptarta. } \\ \text { Limbaria, }\end{array}\right.$

1834 Concinnama, Ramb. $F$. And. . . . Hisp. mer.

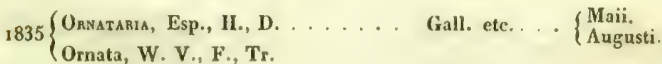

$1836\left\{\begin{array}{l}\text { Decoraria, H., D. . . . . . G Gall, mer. . A A gusti. } \\ \text { Decorata, Tr. } \\ \text { Cinerata, F. }\end{array}\right.$

$\left\{\begin{array}{l}\text { Submutata, Tr. } \\ \text { Contiguaria, D. }\end{array}\right.$

$1838\left\{\begin{array}{l}\text { Immutars, } \text { H., D. . . . . . . . . P., etc. . . . Julii. }_{\text {Immutata, Tr. }} \\ \text { An ab.PAniculosata, Ramb.A.S. Phys. Monspelii. }\end{array}\right.$

$1839\{$ Puerlaria . . . . . Helvetia

84 (Conriguaria, H. (non D.). . . . . Gall. bor.... Julii.

$1840\left\{\begin{array}{l}\text { Contiguaria, H. (non } \\ \text { Contiguata, Tr. Suppl. }\end{array}\right.$ 
$184,\left\{\begin{array}{l}\text { Incanaria, I., D. . . . . . . . . P., etc. .... Augusti. } \\ \text { Var. Virgularia, H. }\end{array}\right.$

3842 Canteneraria (1).......... Galloprov. . Junii.

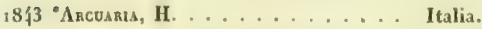

1844 Rugellarta, Ramb. F And..... Hispan, mer.

1845 Incomptara (2), Ramb, $F$, And. . . Gall. mer. . Junii.

$1846\left\{\begin{array}{l}\text { Mrcrosara. } \\ \text { Pusillaria (3), H., Tr, D. . . . . . Gallia, etc. . . Julii. }\end{array}\right.$

$1847\left\{\begin{array}{l}\text { Monuraru, H., D............ Gall. mer. . Julii. } \\ \text { Moniliata, F., Tr. }\end{array}\right.$

计 Alx lucidx; anticx fascia magna fusca; lịn. margin. obsoletis.

${ }_{38} 8$ f $\{$ Frlicaria, D. ........... Gall. mer. . Junii.

I $849\left\{\begin{array}{l}\text { Rosticaria, D. } \\ \text { Rusticata, H. Tr. }\end{array} \ldots \ldots \ldots\right.$ P., etc. .... Jutii.

†† Alæ lucidæ, transversim lineatæ, fascia marginali sæpius obscuriori.

$1850\left\{\begin{array}{l}\text { Scutulså, H. } 72, \text { D: pl, 220.... P., etc. . . J Jilii. } \\ \text { Scutulata, Tr. } \\ \text { Scutata, F. }\end{array}\right.$

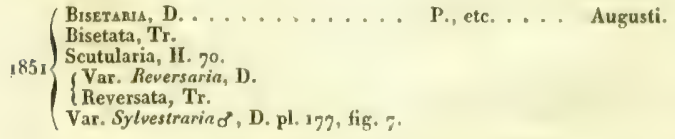

(1) Statura Rusticaria miboris et aflinis Incanaria, at illa brevior et latior. Ala albidx atomis minutissimis obscurioribus, raris, puncto centrali nigro, margine lineis puactularibus duabus confusis fuscis. - In Galloprovincia detecta a Dom. Cantener.

(a) Statura Moniliaria minoris, sub-affiais Mierosarias. Alx omnes albido-fulvae, sub. pulverulenta, fasciis quatuor valde sinuosis, parallelis, strigulaque apicali fuscis ; subtu. immaculata.

(3) Larva Lierbariorum pestis ; plantas slceas corrodit et diruit. 
1852 Politaria, H., Tr., D. ....... Gall, cent. . Junii.

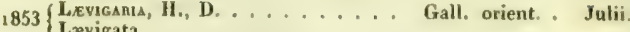
Lrevigata.

$1854^{\circ}$ Renulakia, 11. . . . . . . . Mispan.

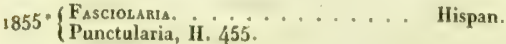

1856 Circurtanta, II. . . . . . . . Galloprov. . Junii.

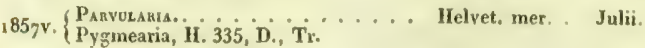

t+t+ Alæ flavæ; fascia marginali rubra.

1858 VitTaria, H., Tr., D. . . . . . Gall. mer. . . Junii.

1859 Ostrinara , H. . . . . . . Galloprov. . Junii.

860 Avrorara, H., Tr., D. . . . . . P., etc. . . . Julii.

Pyr. auroralis, W.V.

t+t†t Alæ lutex vel fuscæ, lincis obscurioribus. G. Acidalin, Dup. - Alæ trilineatæ.

186 1 Fuaveoraria, H., D., Tr. Suppl. ... \{ $\begin{aligned} & \text { Fontisbleaudi } \\ & \text { frequentissima. Junii. }\end{aligned}$

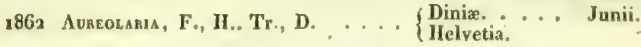

\section{" Alæ multilineatæe.}

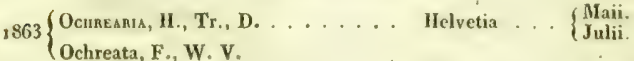

1864 Rufakta, H., Tr., D. . . . . . . Gall. orient. . Julii.

$1865\left\{\begin{array}{l}\text { Paxzrdara, II., Tr., D. . . . . . Europ. Sylvat. Julii } \\ \text { Pallidata, W. V. }\end{array}\right.$

....

1866 Rubricamia, H., Tr., D......... Gall. arid. . Julii,

${ }_{1867}$ Torbidaria, H. ........... Italia.

1868 Ingonspicuaria $(1) \ldots \ldots \ldots . . .$. Hispan.

(1) Cum Osyrarin feinind fere convenit, An eadem P 
-... Alæ pallidx, multilineatæ.

1869 Distanctania (1), Ramb. F. And.... Galloprov. . Junii. 18 go v.Oblitaria, Ramb. $F$. And....... Hispan. mer.

1871 Mhaceliaria, Ramb......... Malaga.. . . Maii.

${ }_{1872}$ Obsoretania (2), Ramb. F. And.... Galloprov. .. Junii. $18 \gamma^{3}$ v. Temeluakia, Ramb. $F$. And. . ... Hispan. mer.

1874 v.Atrendara, Ramb. Ann.S.Ent... \{ $\begin{aligned} & \text { Corsica. } \\ & \text { Gall. austral. }\end{aligned}$

I875 Infirasaru, Ramb. Ann. S. Ent. . . \{ l Galloprov. . Maii.

1876 v.Macilentakis, Ramb. F. And. . Hispan. mer.

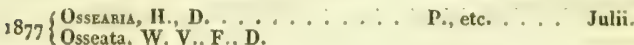

18 8̧8 Palearia, Ramb. F. Aur. . ... Mispan. mer.

$1879\left\{\begin{array}{l}\text { Interjectama. } \\ \text { Dilutaria, H. . . . . . . . . M Mispan. mer. }\end{array}\right.$

$2880\left\{\begin{array}{l}\text { Strananar14, Tr. . . . . . Saxonia. } \\ \text { Straminata. }\end{array}\right.$

t+t+t+ Alæ luteæ vel albæ; strigis solito magis undulatis.

${ }_{188 \text { I }}\left\{\begin{array}{l}\text { Lvteanra, H. , Tr., D. . . . . . E Europ. . . . Maii. } \\ \text { Luteata, W. V., F. }\end{array}\right.$

$1882\left\{\begin{array}{l}\text { Decoloraria. } \\ \text { Decolorata, II }\end{array}\right.$

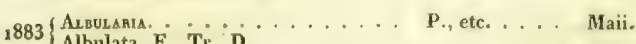

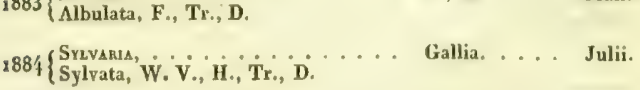

(1) Mioor quam Ossearia. Als pallide cinerese, sub-lucidx, lineis transversis obsoletistimis.

(2) Colore variat; sxpius albido-rufescens. Statura Ossearia minoris. Alm tenuissime pulverulentæ puncto discoidali, punctisque marginalibus nigris, intra medium et apicem lineis quatuor communibus, undulatis, fuscis, - In Galloproviacis frequens. 
1885 Cisndidarı, H., U. ........ Europ, pratis. . Maii.

Candidata, W, V., Tr.

2886r. $\left\{\begin{array}{l}\text { Brssinaria. . . . . . . . . } \\ \text { Byssinata, Tr. Fr. }\end{array}\right.$

$t+t+1+t$ Alæ fuscx, striis albo-sericeis.

188jv.Nexakia, H. .......... Hispan. mer.

3888 Sericearia $\ldots . . .$. Gallia, etc. . Junii.

Sericeata, H., Tr., D.

tt+t+t+t Alæ strigis crassioribus, pulverulentis, fimbriaque intersecta,

1889 lacernarta, H. . . . . . . . Italia.

I89o Glakearu, W. V., Bork,, H., Tr., D. Galt. mer. . \{ Maii.

$1891\left\{\begin{array}{l}\text { Immorania, H., D. . . . . . . . . Gallia in exi- } \\ \text { Immorata, L., Tr. }\end{array}\right.$

$+t++t+t+t$ Alæ pallidæ vel albidx; strigis angustioribus.

$189^{2}$ v. Lutosanta, Ramb. F, And. . . Hispan. mer. Mait.

$183^{3} \cdot\left\{\begin{array}{l}\text { Grammicanı } \\ \text { Sylvestraria, II. } 94 . \cdots \ldots \ldots \text { Patria? }\end{array}\right.$

1894 Strigaria, H., Tr., D. . . . . . . G Gallia. . . . Junii.

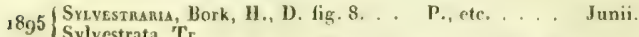

s 896 Adjosctakita (1) . . . . . . . Alp. Lombard. Augusti.

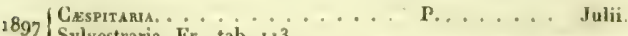

1897 Sylvestraria, Fr, tab. $1 x^{3}$.

(6) Affuis Strigaria; ala sordide pulverulento-slbida, intra medium et spicem, liapis tribus, parallelis, vis undulatis; sigaaloc; linea interiori fere recta. Also omnes aublus mitidiores; antic lineis duabus, postice tribus, signatar. - In Italia boreali detecta a D. Bar. Feisthamel. 
${ }_{189} \mathrm{~s}\left\{\begin{array}{l}\text { Pexctarla, Devill , Tr., D. . . . . } \\ \text { Nemoraria, H. }\end{array}\right.$ $18_{99}$ Litigiosaria (1), Ramb. $F$. And. . . Gall. mer. . Junii, syoov.Neglectaria, Ramb. F. And. . . . Hispan. mer. 1 go Elongaria, Ramb. Ann. S. Ent. ... $\begin{aligned} & \text { Corsica...... Maii. } \\ & \text { Galloprov. }\end{aligned}$ 1902 v. Modestaria, Ramb. F. And. . . . . Hispan, mer.

1903 Perfluara (2), Ramb. $\boldsymbol{F}$. And.... Monspelii. . Maii. 1904 Nitidaria (3). . . . . . . . . . Lapponia.

\$go5 GYPSARIA (4)........... Germ. bor. I906 $\left\{\begin{array}{l}\text { Commotaria. . . . . . . . } \\ \text { German. . . Junii. } \\ \text { Helvetia. }\end{array}\right.$

Commutata, Tr., Fr. tab. 77.

9907 Remutara, H., D. . . . . . . P., ete ... Junii.

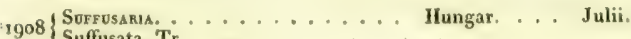
tttfttttt Alæ sinuatx; fascia media late infuscata.

1909 Degemerarta, H., Tr., D. . . . . . Gallia. . . . Junii.

$1910\{$ Aversaria, II., D. ......... P., etc. .... Julii. Aversata, L., Tr., etc.

ttt+tt+tt+ Alæ rufæ, sinuato-emarginatæ.

$1913\left\{\begin{array}{l}\text { Emarginaria, II., Tr., D....... P., etc.... Julii. } \\ \text { Emarginata, L } \\ \text { Demandata, I. }\end{array}\right.$

(1) Statura Cespitarie. Alæe ombes sordide albido-pulverulentoe, puncto discoidali nigro, intra medium et marginem, lineis tribus undulatis fuscis; duabus externis approximatis; als anticæ ante punctum nigrum, linea fusca sub-undulats signatx.

(2) Statura Sylvestraria. Alæ tenell a, albe, intra medium et fimbriam, libeis quatwor fuscis, teoue undulatis, esterna marginali obsoleta.

(8) Sub-aftinis Remutaria, alis anticis minus ad a picem rotundatis. Alae omnes cinereo-albx; pulverulenta; antica lineis tribus, posticue duabus, in medio locatis, aque distantibus, haud undulatis, signatae.

( Iribus vis undulatis fuseis, externa obsoleta sub-margioali, penultima sub-irregulari. Vemina rate paulo minor, - Hane novam speciem accepi e Germania a Cl. Demel. 
Htttftt+tt Alx posticre angulatr.

- Alæ fulvæ.

2912 Imitaria, H., Tr., D. . . . . . . G Gall. mer. . Julii.

" Alæ albida.

1913 Emutarta, H., Tr., D. . . . . . G Gall. mer. . Junii

1914 Mediaru, H. . . . . . . . . Galloproy. . Maii

I9I5 $\left\{\begin{array}{l}\text { Muratakia, H., D. . . . . . . Gall. Alp... Julii. } \\ \text { Mutata, Tr. }\end{array}\right.$

1916 Umbelaria; H. . . . . . . . Gall. cent. . Junii.

$1917\left\{\begin{array}{l}\text { Pratara. } \\ \text { Strigilaria (1), H., D. } \\ \text { Strigilata, W. V., Tr. }\end{array}\right.$

\author{
5o. GENUS TIMANDRA, Dup. \\ Bradyepetes, Steph. Ennomos, Tr.
}

Larva abbreviata, antice incrassata, rigida, herbicola. Metamorphosis sub-folliculata. - Antennæ maris pectinatx. Palpi longiores. Lingua brevis. Alæ patulæ, striga communi diagonali signatæ; antic apice acutæ; posticæ angulatæ. Statura sub-media.

Hocce genus ab Acidaliis angulosis facile distinguitur larva brevissima, antennisque maris pectinatis.

1918 Amataru, L. H., etc. ....... P., etc.... \{ Maii.

\title{
53. GEIVUS STRENIA, Dup.
}

Fidonia, Tr. Macaria, Curtis.

Iarvæ attenuatx, læves, cylindricx. Metamorphosis hypogæa. Antennæ filiformes. Palpi brevissimi. Lingua producta. Alæ utrinque concolores, strigato-cancellata, per quietem erectx.

Hoc genus differt a Fidoniis, antemis setiformibus in utroque sexu, et ab Acidaliis cohortis Glarearia, alis per quietem erectis.

(1) Nomen Strigilaria nimis simile nomini Strigillerix, quare mutandum. 


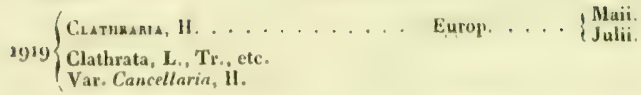

1920 Tessermata (1)............ Italia bor.

52. GENUS SIONA, Dup

$$
\text { Idea, Tr. (2). }
$$

Larve ignota. - - Antenna simplices. Palpi acuti, ultra clypeum assuigentes $\Lambda$ la integra vel denticulata, concolores, patula, nervis evidentioribus, albidx, vel fusce, striga unica. - Volatus diurnus. Statura major.

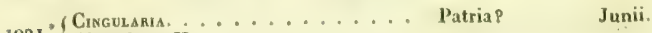

1921 (Cingulata, H.

1922 " Renitidara. .............. Patria?

1923 Dealbama, H. .......... P., etc. ... Junii.

$19^{23}$ Dealbata, L., etc.

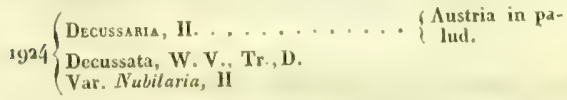

$392.5 \%\left\{\begin{array}{l}\text { Exalbara fan var, præced.P). . . . Hungar. } \\ \text { Exalbata, 11. }\end{array}\right.$

$1927\left\{\begin{array}{l}\text { Grisearta, H. } \\ \text { Griseata, W. V., Tr, D. . . . Diniæ, etc. . Junii. }\end{array}\right.$

1928 A sisaria. if ........ Russ. mer.

1929 Tenebrark, II. . . . . . . Hungaria.

1930 Fumdarla, H., Tr. ......... Hungaria.

(1) Specios distinclissima. Stotura Clathrarix; alx utrinque nigro-fuscur, albo tessellate: fimbria nigea albo intersecta. Aateone crassiores tenue dentato. In Italia borcali drtexit D, Baro Fejsthamel.

(2) Nomen Iden jamdiu ud aliud Genus Lepisloplerorum tribuil Celob olatseille, guare nomen Treitssbianum rejiciendum. 


\section{GENUS STHANELIA.}

Chesins, Tr., Dup.

Iarva elongatx, laves, Ericicole; capite crassiusculo. Metanorphosis hypogza, - Antennæ simplices. Palpi mediocres, apice contigui. lingua producta. Tibix postice incrassatx. Alx sub-tectiformes, antice elongat $x$, signaturis obsoletis.

$1931\left\{\begin{array}{l}\text { Fuscarza, Thunb , H. . . . . . . . . Teptembr. } \\ \text { Tibiaria, Ramb. Ann, S. Phys., D. }\end{array}\right.$

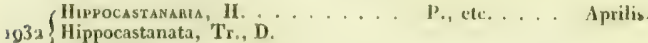

Var.? Degenerata, H. 405.

54 GENUS ODEZZIA.

Psodos, Tr. Tanagra (1), Dup.

Larvæ teuues, elongata, laves. Puppa sub-folliculata. - Antenne simplices. Palpi breviusculi, hirsuti. Lingua producta. Alæ per quietem erectre, integerrimæ, nigræ. Statura debilis, sub-media.Volatus diurnus.

+ Nax immaculatx.

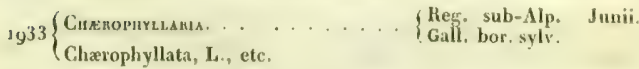

t† Macula alba in alis anticis.

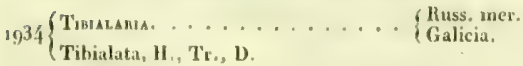

(1) Momen Tanagra in Ornitbologia jam usurpulum cal al aliud genus designandur. 


\section{GENUS TORULA.}

$$
\text { Psodos, Tr., Dup. }
$$

Larva ignota. - Antennæ simplices. Palpi villosissimi, setosi, ultra clypeum assurgentes. Lingua producta. $\Lambda$ læ per quietem erectæ, integerrimæ, nigræ, macula lutea. Statura debilis, sub-media. - Volatus diurnus.

$1935\left\{\begin{array}{l}\text { Equestraria, Esp., Fab. . . . . . Alpes. . . J Julii. } \\ \text { Alpinata, W. V., Tr., D. }\end{array}\right.$

\section{GENUS PSODOS.}

Psodos, Tr., Dup.

Metamorphoses ignotx.-Antennæ simplices. Palpi villosissimi, setosi, ultra clypeum assurgentes. $\Lambda$ læ integerrimæ, per quietem sub-patulæ, nigro-fuscæe, puncto discoïdali fasciaque intra-marginali obscurioribus. Statura sub-media, robustior.

Hocee genus ad Pyralidiss generis Mercyna transitum efformatr: videtur.

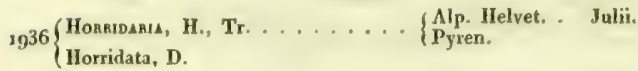

$19^{3} 7\left\{\begin{array}{l}\text { Trepidani, H., Tr. . . . . . A Alp. Helvet. Julii. } \\ \text { Trepidata, D. }\end{array}\right.$

1938 Chuosanu, Frey. Beye tab. 36. . A Alp. Helvet. A Augusti.

\section{GENUS PYGM/ENA. \\ Psodos, Tr., Dup.}

Metamorphosis ignota. - Antennx maris pectinatx. Palpi breviusculi, villosi. Lingua brevis. Al $x$ patulx, integræ, nigræ. Statura minima.-Volatus maris diurnus.

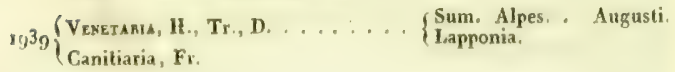


58. GENUS ANTHOMETRA, Rambur.

Vetamorphosis ignota. - Antenne maris breves, plumoso-pectinatic, latiores. Lingua brevis. $\Delta l x$ integerrim $x$, ruf $x$, immaculat $x$. Staturit minima, - Volatus maris meridianus.

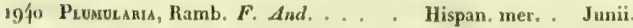

59. GENUS MINUA, Dup.

Minon, Tr., Curtis.

Larva medio inflata, utrincque attenuata, pilosula; capite globuloso, minuto. Metumorphosis sub-folliculata. - Antennx simplices. Palpi brevissimi. Alie integerrim $x$, unicolores, cincrex, immaculat $x$. Volatus sub-diurnus. Statura minima.

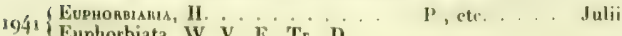

Euphorbiata, W. V., F., Tr., D.

ฟNIล. 



\title{
INDEX ALPHABETICUS
}

\author{
GENERUM.
}

\author{
OBSERVATIO.
}

NoMINA SYSTEMATICA B.ITEERIS ROMANIS, SYNOXYHA LITTEMIS ITALCIS, DOTATA SUNT.

ged

Abrostola, 15\%.

decena, $18 \mathrm{t}$.

Achatea, 142 .

Acherontia, 49 .

Acidalia, 221.

Acontia, 165 .

Acronycta, 94.

Egeria, 41.

Aglia, 74 .

Agriopis, 123.

Agrophila, 175 .

Agrotis, 108.

Amnthia, 207.

Amphidasis, 395 .

Amphipyra, 99.

Anaitis, 20\%.

Anarta, 161.

Anisopteryx, 393.

Biston, 195.

Boarmia, 196 .

Boletobia, 201.

Bombyx, 6n.
$\Lambda$

Anthocharis, 5.

Anthocia, 162.

Anthophila, $1-3$.

Anthometra, 231

Anthrocera, 50.

A pamea, 16 .

Apatela, 94 .

A patura, 24.

Aplecta, 122.

Arctia, 64 .

Arcturus, 67.

Arge, 25.

Argznnis, 17.

Aspilates, 187

Asteroscopus, 85 .

Altacus, 73 .

Atychic, 40, 5 1.

Aventia, 185.

B

Brephos, 172.

Brithya, 117.

Bryophila, 96 . 
i

Cabera, 288.

Callimorpha, 61 .

Calocampa, 149

Calpe, 156 .

Calyptra, 98 .

Caradrina, 136.

Catephia, 166.

Catocala, ${ }_{1} 67$.

Cerastis, 147 .

Cerigo, 101.

Cerocala, 171 .

Ceropacha, 92.

Cerura, 84 .

Chaonia, 87.

Chariclea, 152.

Chariptera, 123.

Chelonia, 63.

Chemerina, 193.

Chersotis, 103.

Chesias, 212 .
Charaxes, 24.

Chimara, 40.

Chionobas, 29

Chrysoptera, 157.

Cidaria, 212.

Cilix, 82.

Cleoceris, 93.

Cleogene, 3,8

Cleophana, 151 .

Clidia, 68.

Clisiocampa, 60.

Cloantha, 150.

Clostera, 89 .

Cnethocampa, 6 g.

Cocytia, 117.

Colias, 7.

Cosmia, 143.

Cossus, -5 .

Crocallis, 184.

Cucullia, 152.

Cymatophora, 92 .

1)

Danais, 5 .

Dasycampa, 147

Dasychira, 67.

Dejopein, 56.

Demus, 67 .

Deilephila, $\$ 4$;.
Dianthacia, 224.

Dicranura, 84 .

Diloba, 88.

Diphtera, g:.

Dorvilia, 74.

Drepana, 82 .

Fi.

E'rioguster, 60

liriopus, 129.

Eubolia, 201.

Euchelia, 56.

Euclidia, 172.

Eulepia, 56.

Eupisteria, 192.

Eupithecia, 208.

Eurhipia, 122.

Eyprepia, 57-65. 
F

Fidonia, 190.

Fumea, 78 .

G

Gastropacha, 69).

Geometra, 179

Glaea, 147 .

Glottula, 117

Gluphisia, 88 .

Gnophos, 199.

Gonoptera, 98

Gortyna, 144.

II

Hadena, 117.

Ifamerosia, 163.

Halia, 187 .

IIamearis, 14.

Harpya, 85.

Heliophobus, I11.

Heliothea, $8>8$.

Heliothis, 162 .

Ilemithea, $1-9$

IIepialus, 76 .

Hepiolus, 76 .

Hesperia, 34 .

Heterogenea, 8 .

Heterogynis, 55 .

libernia, 194.

limera, 184 .

Hoporina, 847.

Hydrilla, 138.

Hypercomipa, 6 I.

Нypogymua, 66.

I et J

Ilarus, 125

Jaspidia, 128 .

1

Lolia. 67.

Larentia, 20 f

Lasiocampa, 72

Leiocrmpa, 86.

Leucania, 131.

Leucophasia, 6 .

Libythea, 23.

ligia, 189 .

Limacodes, 81

Limenitis, 16.

Liparis, 66.

Lithosia, 5 7.

Lobophora, $20 \%$

Lophoptery $\boldsymbol{x}, 86$.

Luperina, 113

Lyçena, 10.

M

Macaria, 186.

Macroglossa, 45

Megasoma, 72 .

Melanippe, 215

Mania, 100.

Melanthia, 2 , 6 
Melasina, 57 .

Melitaa, 19.

Mesogona, 144.

Metroeampa, 181.

Miпиа, 136.

Microphisa, 170.

Minoa, 231.

Miselia, 123.

Mniophila, 200.

Mythimna, 31 .

N

Vaclia, 6o.

Nemeobius, 14.

Nemeophila, G2.

Neptis, 16.

Noetua, 104.

Nonagria, 134.

Nolodonta, 86 .

Nudaria, 60.

Numeria, 190.

Nymphalis, 86.

Nyssia, 194.

()

Odezia,"220.

Odonestis, $7 \mathrm{x}$.

Ophiusa, 160.

Opigena, 103.

Orthorhinia. 86.

Orthosia, 140 .

Ourapteryx, 181 .

p

Paniz, 84

Papilio, 1 .

Parnassius, 3 .

Pellonin, 187 .

Pentophera, 66.

Periden, 87.

Petasia, 85.

Philobin, 186.

Philopyra, 99.

Phlogophora, 121.

Phorodesma, I79.

Phragmalobia, 65

Phyllometra, 193.

Pieris, 4.

I'lacodes, 129.

Plastenis, $9^{3}$.

Platypteryx, 8z.

Ploseria, 190.

Plusia, 158.

Pecilia, 96.

Pocilocampa, 60.

Polia, 126.

Polyommatus, 9 .

Polyphanis, 128.

Pontia, 4-6.

Procris, 54.

Psilarn, 56. 66

Psodos, 23o.

Psyche, 78 .

Pterogon, 46.

Ptilodontis, 86.

Ptilophora, 88.

Pygæera, 88.

Pygmæna, 230.

Pyrophila, 49. 
IR

Ahodocern, 6.

Humiz, $18 \%$.

Saturnia, 73.

Satyrus, 30.

Scodiona, 185.

Scotophila, 99 .

Segetia, 101.

Sericaria, 88.

Sesia, 41.

Selina, 59 .

Simyra, 136

Siona, 228.

Smerinthus, 49 .

Spatotis, yo6.

Teplirosia, 198.

Tether, 93 .

Thais, 2.

Thanaos, 3\%.

Thecla, 8.

Thetidia, 180.

Thyatyra, 13o.

Thyris, 4 r.

Urapteryx, $18 \mathbf{I}$

Taleria, 124.

Xanthice 145 .

Xy firisa 11!
Rusing, 100 ,

s

Speranza, 192.

Sphinx, 48.

Spilosoma, 64.

Spintherops, 98 .

Struropus, 85.

Steropes, 3 f.

Sthanelia, 229 .

Stilbia, 356 .

Strenia, 227.

Stygia, 39 .

Syntomis, 54

Syrichthus, 35.

T

Timandra, 227.

Timia, 173 .

Torula, 230 .

Trachaa, 142.

Prichiura, 69 .

Trichosoma, iz. $_{2}$

Triphena, 10 '.

Typhonia, 78.

U

Uropus, 85.

V

Vanessa, 21.

X 
$\mathbf{X}$

Xanthia, I13.

Xerene, 218.

Xylina, 149 .

Xylocampa, 150.

Xylophasia, 113.

Z.

Zegris, 6 .

Zerene, 218.

Zerynthia, 2.

Zeuzera, 75 .

Zygana, -ast $<0$

EX TYPIS FAIN ET THUNOT,

Via Racine; 28. 


(2)

SW

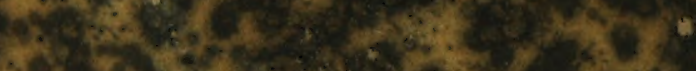

